\title{
Non-Invasive Imaging of the Carotid Artery
}

Citation for published version (APA):

Schreuder, F. H. B. M. (2021). Non-Invasive Imaging of the Carotid Artery: From structural vessel wall to functional plaque imaging. [Doctoral Thesis, Maastricht University]. Maastricht University. https://doi.org/10.26481/dis.20210121fs

Document status and date:

Published: 01/01/2021

DOI:

$10.26481 /$ dis.20210121fs

Document Version:

Publisher's PDF, also known as Version of record

\section{Please check the document version of this publication:}

- A submitted manuscript is the version of the article upon submission and before peer-review. There can be important differences between the submitted version and the official published version of record.

People interested in the research are advised to contact the author for the final version of the publication, or visit the DOI to the publisher's website.

- The final author version and the galley proof are versions of the publication after peer review.

- The final published version features the final layout of the paper including the volume, issue and page numbers.

Link to publication

\footnotetext{
General rights rights.

- You may freely distribute the URL identifying the publication in the public portal. please follow below link for the End User Agreement:

www.umlib.nl/taverne-license

Take down policy

If you believe that this document breaches copyright please contact us at:

repository@maastrichtuniversity.nl

providing details and we will investigate your claim.
}

Copyright and moral rights for the publications made accessible in the public portal are retained by the authors and/or other copyright owners and it is a condition of accessing publications that users recognise and abide by the legal requirements associated with these

- Users may download and print one copy of any publication from the public portal for the purpose of private study or research.

- You may not further distribute the material or use it for any profit-making activity or commercial gain

If the publication is distributed under the terms of Article $25 \mathrm{fa}$ of the Dutch Copyright Act, indicated by the "Taverne" license above, 




\section{NON-INVASIVE IMAGING OF THE CAROTID ARTERY}

FROM STRUCTURAL VESSEL WALL TO FUNCTIONAL PLAQUE IMAGING

Floris H.B.M. Schreuder 
The research in this thesis (Plaque At RISK study) was financially supported by the Center for Translational Molecular Medicine (CTMM; grant 01C-202) and by the Dutch Heart Foundation (DHF-2008-T094).

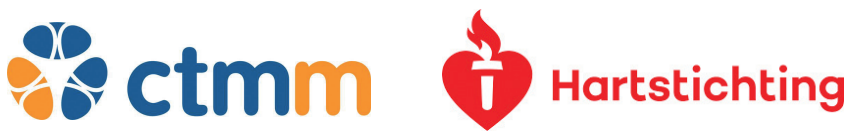

Financial support by the Dutch Heart Foundation for publication of this thesis is gratefully acknowledged.

ISBN: 978-94-6421-170-2

Copyright (c) Floris Schreuder, Nijmegen 2020.

All rights reserved. No part of this publication may be produced, stored in a retrieval system, or transmitted, in any form, or by any means, electronically, mechanically, by print, photo print, recording or any other means without the prior written permission by the author.

Cover and graphic design by Rixt Wieringa

Printed by Ipskamp Printing, The Netherlands 


\title{
NON-INVASIVE IMAGING OF THE CAROTID ARTERY
}

\section{FROM STRUCTURAL VESSEL WALL TO FUNCTIONAL PLAQUE IMAGING}

\author{
PROEFSCHRIFT \\ ter verkrijging van de graad van doctor aan de Universiteit Maastricht, \\ op gezag van de Rector Magnificus, Prof. dr. R.M. Letschert \\ volgens het besluit van het College van Decanen, \\ in het openbaar te verdedigen \\ op donderdag 21 januari 2021 om 14:00 uur \\ door
}

FLORIS HENDRICUS BENJAMIN MICHEL SCHREUDER Geboren op 29 juli 1983 te Geleen 


\section{Promotores}

Prof. dr. W.H. Mess

Prof. dr. M.E. Kooi

Prof. dr. R.J. van Oostenbrugge

\section{Beoordelingscommissie}

Prof. dr. W.H. Backes (voorzitter)

Prof. dr. H. ten Cate

Prof. dr. C.L. de Korte (Radboud Universiteit Nijmegen)

Prof. dr. C.J.M. Klijn (Radboud Universiteit Nijmegen)

Prof. dr. G.W.H. Schurink 
Voor Nienke 



\section{TABLE OF CONTENTS}

Abbreviations

Introduction

Measurement of common carotid artery intima-media thickness in
clinical practice: comparison of B-mode and RF-based technique

3 Wall irregularity rather than intima-media thickness is associated

with nearby atherosclerosis

4 Spatial distension variations are associated with focal

atherosclerotic plaques

5 Orthogonal B-Mode Evaluation of Common Carotid Artery Plaques

Reveals the Absence of Outward Remodeling

6 Ageing affects the accuracy of duplex ultrasonography in grading

107 carotid artery stenosis

7 Simultaneous investigation of structural and functional parameters of carotid artery plaque using routine B-mode ultrasonography: the Plaque At RISK (PARISK) study

8 Development of a diagnostic prediction score to estimate the probability of intraplaque haemorrhage at carotid magnetic resonance imaging in patients with recent cerebrovascular disease: the Plaque At RISK (PARISK) study

9 General discussion

Summary

Samenvatting

Valorisation

Dankwoord

List of publications

Curriculum vitae 



\section{LIST OF ABBREVIATIONS}

$\begin{array}{ll}\text { 95\%-Cl } & \text { 95\% confidence interval } \\ \text { A-mode } & \text { Amplitude mode } \\ \text { AD } & \text { Absolute Distension } \\ \text { B-mode } & \text { Brightness mode } \\ \text { BMI } & \text { Body Mass Index } \\ \text { BP } & \text { Blood Pressure } \\ \text { CAPIAS } & \text { Carotid Artery Plaque Imaging in Acute Stroke study } \\ \text { CCA } & \text { Common Carotid Artery } \\ \text { CCC } & \text { Concordance Correlation Coefficient } \\ \text { CEA } & \text { Carotid EndArterectomy } \\ \text { COG } & \text { Center Of Gravity } \\ \text { CV } & \text { Coefficient of Variation } \\ \text { CVD } & \text { CardioVascular Disease } \\ \text { CTA } & \text { Computer Tomography Angiography } \\ \text { CTCM } & \text { Center for Translational Molecular Medicine } \\ \text { DBP } & \text { Diastolic Blood Pressure } \\ \text { DICOM } & \text { Digital Imaging and Communications in Medicine } \\ \text { e.g. } & \text { Exempli Gratia, "for example" } \\ \text { ECA } & \text { External Carotid Artery } \\ \text { ECG } & \text { ElectroCardioGram } \\ \text { ECST } & \text { European Carotid Surgery Trial } \\ \text { EDV } & \text { End-Diastolic Velocity } \\ \text { FDG } & \text { 18F-fluoro-2-Deoxy-D-Glucose } \\ \text { FS } & \text { Framingham Score } \\ \text { FVS } & \text { Framingham Vascular Score } \\ \text { GS75 } & \text { 75 } \\ \text { GSM } & \text { Grey-Scale Median } \\ \text { HDL } & \text { High Density Lipoprotein } \\ \text { HE } & \text { Haematoxylin Eosin } \\ \text { HR } & \text { Hazard Ratio } \\ \text { Hz } & \text { Hertz } \\ \text { i.e. } & \text { Id Est, "in other words" } \\ \text { ICA } & \text { Internal Carotid Artery } \\ \text { ICC } & \text { Intraclass Correlation Coefficient } \\ \text { IJV } & \text { Internal Jugular Vein } \\ \text { IMT } & \text { Intima-Media Thickness } \\ \text { IPH } & \text { IntraPlaque Hemorrhage } \\ \end{array}$




$\begin{array}{ll}\text { IQR } & \text { Inter Quartile Range } \\ \text { IR-TFE } & \text { T1-weighted Inversion Recovery Turbo Field Echo } \\ \text { LDL } & \text { Low Density Lipoprotein } \\ \text { LRNC } & \text { Lipid-Rich, Necrotic Core } \\ \text { M-line } & \text { M-mode line } \\ \text { M-mode } & \text { Motion mode } \\ \text { MHz } & \text { MegaHerz } \\ \text { mmHg } & \text { Millimeters of mercury } \\ \text { MRA } & \text { Magnetic Resonance Angiography } \\ \text { MRI } & \text { Magnetic Resonance Imaging } \\ \text { MUMC } & \text { Maastricht University Medical Center } \\ \text { n } & \text { Number } \\ \text { NASCET } & \text { North American Symptomatic Carotid Endarterectomy Trial } \\ \text { NNT } & \text { Number Needed to Treat } \\ \text { OR } & \text { Odds Ratio } \\ \text { PARISK } & \text { Plaque At RISK study } \\ \text { PET } & \text { Positron Emission Tomography imaging } \\ \text { PL } & \text { Plaque Length } \\ \text { PSV } & \text { Peak-Systolic Velocity } \\ \text { PT } & \text { Plaque Thickness } \\ \text { PWV } & \text { Pulse Wave Velocity } \\ \text { QIR-TSE } & \text { T1-weighted Quadruple Inverse Recovery Turbo Spin Echo } \\ \text { r } & \text { Correlation coefficient } \\ \text { RD } & \text { Relative Distension } \\ \text { RF } & \text { Radio Frequency } \\ \text { ROI } & \text { Region-Of-Interest } \\ \text { SBP } & \text { Systolic Blood Pressure } \\ \text { SD } & \text { Standard Deviation } \\ \text { SEM } & \text { Standard Error of Mean } \\ \text { SMART } & \text { Second Manifestations of Arterial disease study } \\ \text { SPGR } & \text { T1-weighted Spoiled Gradient echo } \\ \text { T } & \text { Tesla } \\ \text { TIA } & \text { Transient Ischemic Attack } \\ \text { TOF } & \text { Time-Of-Flight } \\ \text { TRFC } & \text { Thin and/or Ruptured Fibrous Cap } \\ \text { TRIPOD } & \text { Transparent Reporting of multivariable prediction model for Individual } \\ \text { US } & \text { Prognosis Or Diagnosis } \\ \text { VA } & \text { Ultrasonography } \\ \text { WSS } & \text { Vertebral Artery } \\ & \text { Wall Shear Stress } \\ & \end{array}$






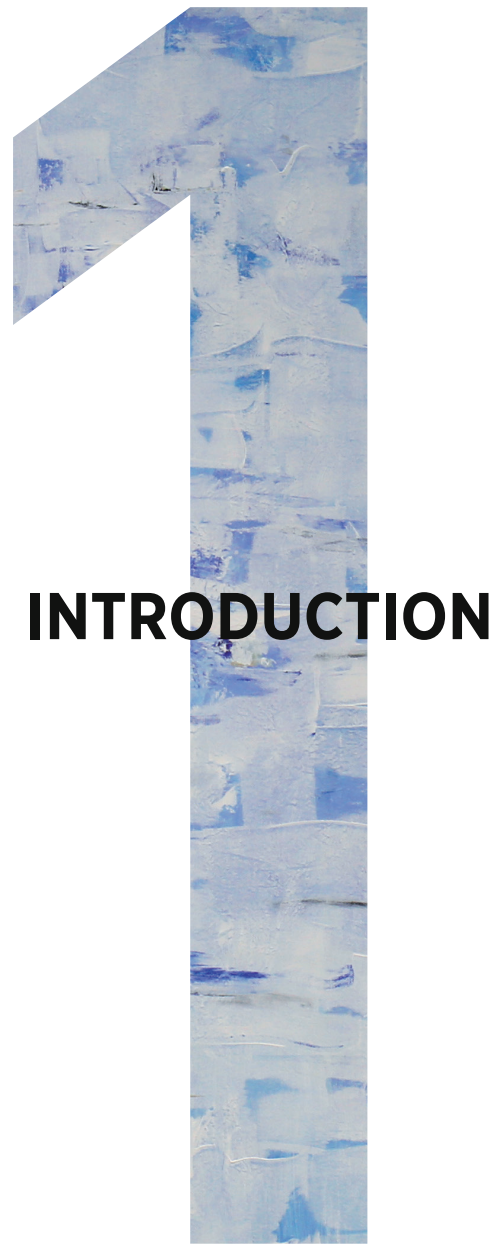


* This case report was adapted from Kwee RM, Schreuder FH, Mess WH, van Oostenbrugge RJ, et. al. Images in cardiovascular medicine: development of carotid intraplaque hemorrhage demonstrated by serial magnetic resonance imaging. Circulation 2009; 120(16): 1637-39 


\section{Case report *}

A 68-year-old man presented with a sudden onset of weakness and paresthesia of the left arm. He had a history of myocardial infarction and renal artery stenosis. A nonenhanced computed tomographic scan of the brain showed no signs of hemorrhage or recent infarction. The patient underwent intravenous thrombolysis with alteplase, after which symptoms disappeared except for the persistence of a slight paresthesia of the left arm. Vessel ultrasound showed a hypo-echoic plaque in the right carotid artery bifurcation causing a $50 \%$ to $69 \%$ stenosis, with normal blood flow velocities at Doppler measurement. Thus, this stenosis was considered to be non-significant. Extensive analysis revealed no other cause for embolic stroke. In addition to the aspirin (100 mg once per day) and atorvastatin (40 mg once per day) he was already using, the patient was prescribed dipyridamole (200 mg twice per day) for secondary stroke prevention. Fifteen days after the initial onset of symptoms, the patient was enrolled in a clinical study investigating the natural history of mild to moderate carotid artery stenosis.' Magnetic resonance imaging scans of the brain and right carotid artery plaque showed a recent discrete infarction of the right sensory region (Figure 1.1) and a plaque with a large lipid-rich necrotic core with no or little hemorrhage (Figure 1.2 panel A-D), respectively.

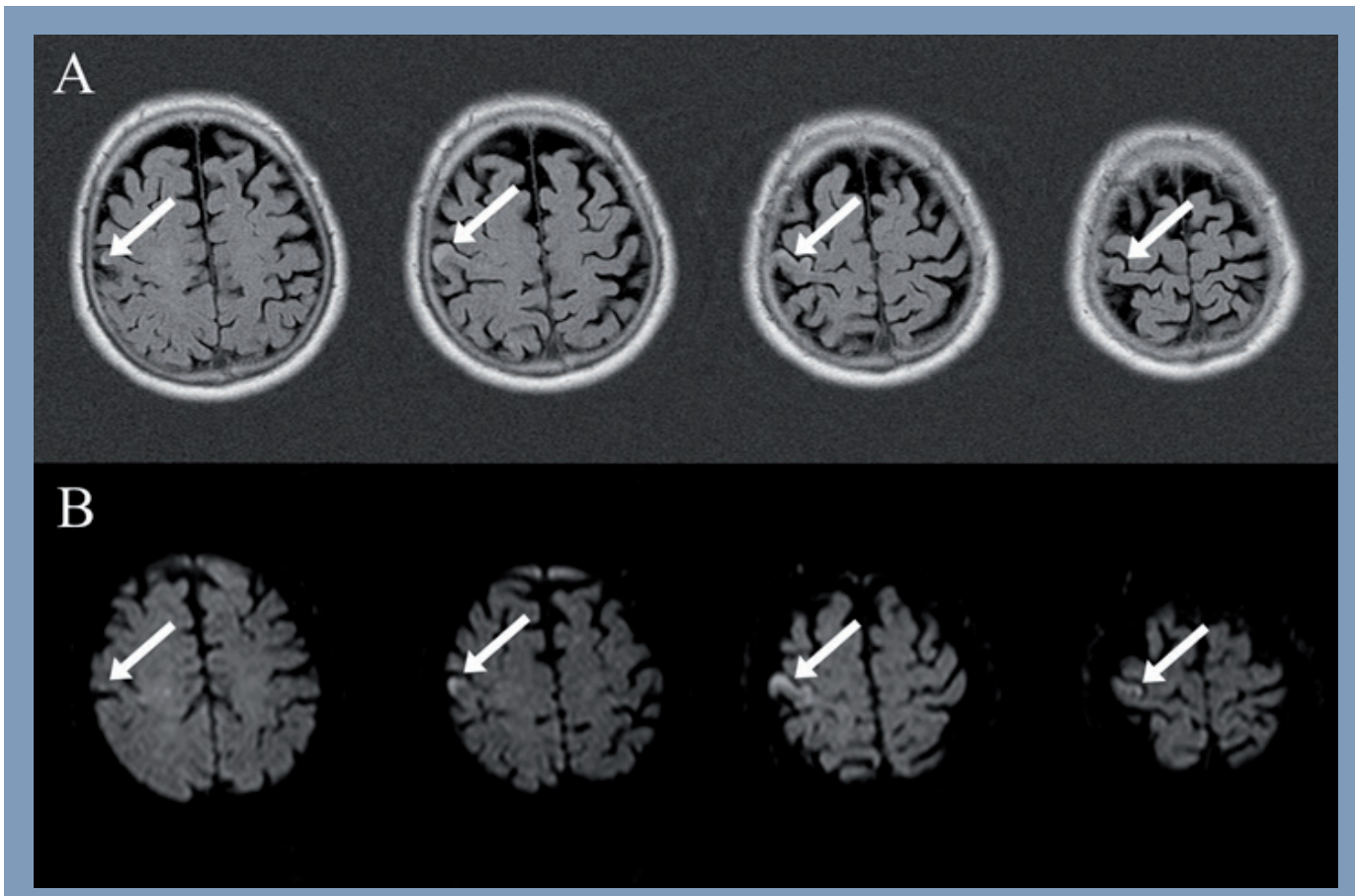

Figure 1.1. Baseline cross-sectional fluid-attenuated inversion recovery (A) and diffusion-weighted (B) images of the brain show a very small hyperintense area in the right sensory region (arrows), indicating recent discrete infarction. 

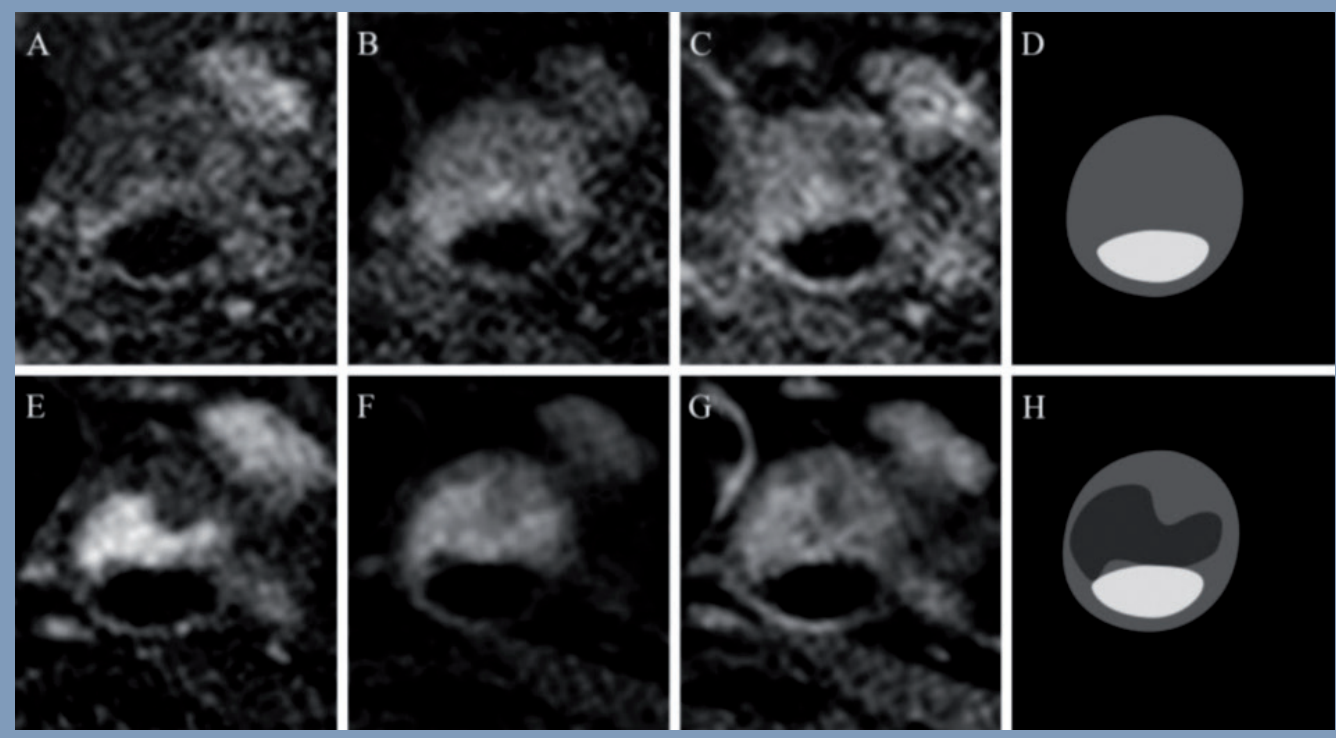

Figure 1.2. 1.5-Tesla magnetic resonance image, with cross-sectional co-registered images through plaque in the right internal carotid artery. Baseline T2-weighted image (A) and precontrast (B) and postcontrast (C) T1-weighted images and plaque contours (D) (white: carotid artery lumen; light gray: vessel wall area) are shown. T2-weighted image (A) shows isointense plaque, precontrast T1-weighted image (B) shows hyperintense plaque, and postcontrast T1-weighted image (C) shows no enhancement of the plaque, which matches the criteria of a lipid-rich necrotic core with no or little hemorrhage. Follow-up T2-weighted (E) and precontrast $(F)$ and postcontrast (G) T1-weighted images and plaque contours (F) (white: carotid artery lumen; light gray: vessel wall area; dark gray: hemorrhage) are shown. T2-weighted image (E) shows a striking hyperintense area within the plaque, adjacent to the lumen (dark gray area in H). This area is hyperintense at the precontrast T1-weighted image (F) and does not enhance at the postcontrast T7weighted image (G). These findings indicate recent intraplaque hemorrhage.

Three months after the initial event, the patient once more presented with sudden onset of weakness and a numb feeling of the left arm. The patient again underwent intravenous thrombolysis with alteplase. However, the symptoms persisted. Repeated Doppler ultrasonography showed no change in carotid plaque echogenicity and comparable degree of luminal narrowing of the right internal carotid artery. Ten days after the onset of the second event, the patient underwent repeated magnetic resonance imaging scans of the brain and right internal carotid artery plaque. These scans confirmed recent infarction in the right motor region (Figure 1.3) and revealed recent intraplaque hemorrhage (Figure 1.2 panel E-H).

Because of recurrent symptoms, the patient underwent carotid endarterectomy 20 days after the onset of the second event. Histological analysis of the excised plaque demonstrated large intraplaque hemorrhage (Figure 1.4), confirming the magnetic resonance imaging findings. 


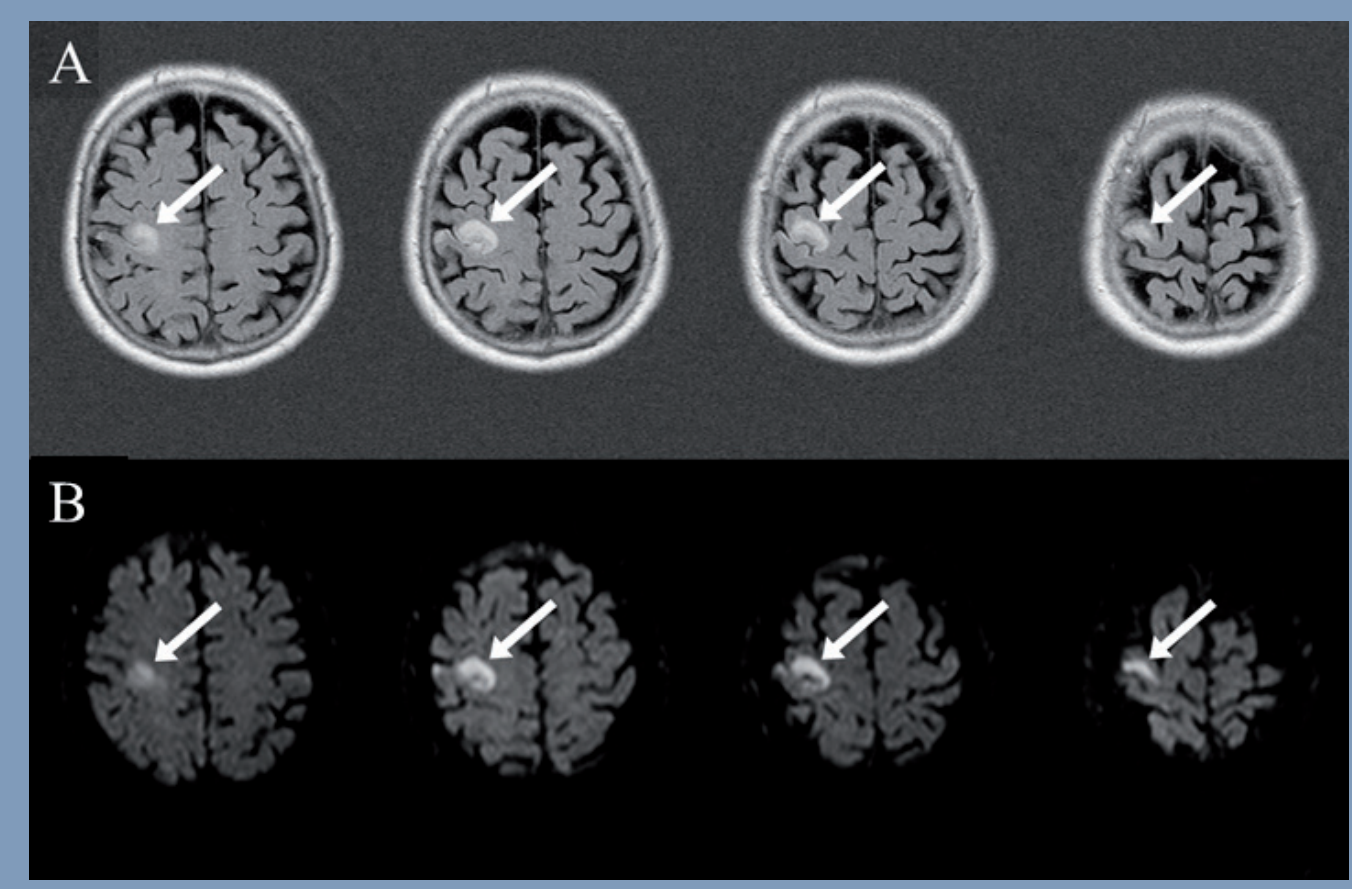

Figure 1.3. Follow-up cross-sectional magnetic resonance images of the brain. Fluid-attenuated inversion recovery $(A)$ and diffusion-weighted $(B)$ images now clearly show a new hyperintense area in the right motor region (arrows), indicating recent infarction.

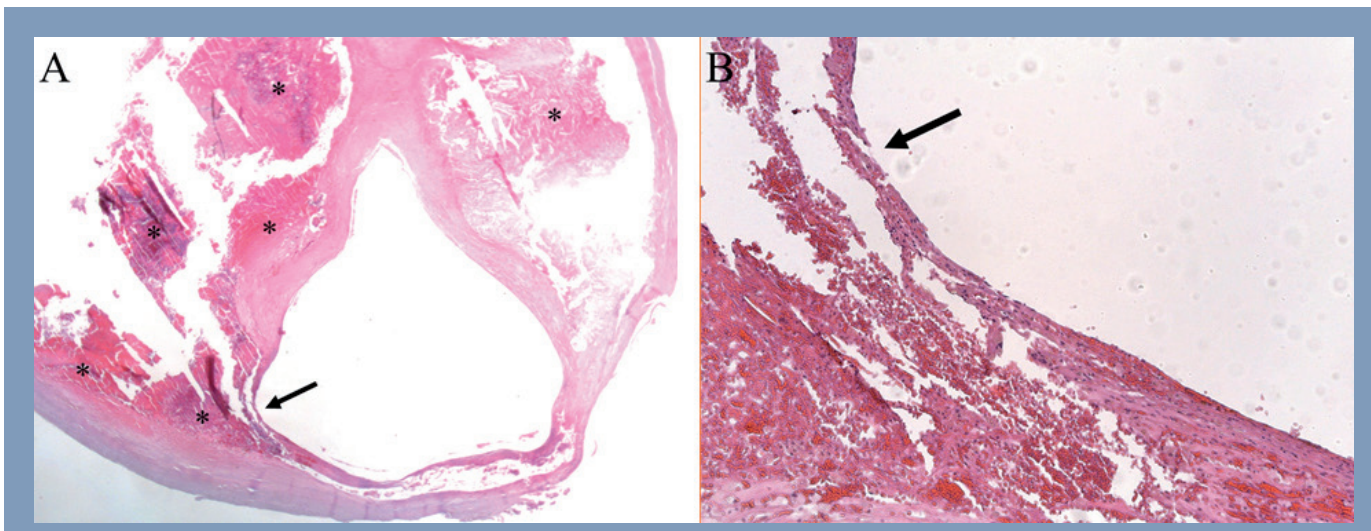

Figure 1.4. Cross section through the carotid endarterectomy specimen, corresponding to the level of the magnetic resonance images (Figure 1.2). Hematoxylin-eosin staining; magnification at x125 (A) and x1000 (B). There are large areas of intraplaque hemorrhage (asterisks in A). At the right shoulder region, the fibrous cap is very thin and shows recent reendothelialization (arrows in A and B). Many erythrocytes are present within the fibrous cap (B). 
This clinical case illustrates that stroke recurrence is a significant problem despite maximal medical treatment. Additionally, it shows failure of current diagnostic strategy used for risk stratification and also demonstrates the potential of alternative diagnostic strategies. In this introduction, ischemic stroke pathophysiology, the vulnerable plaque concept and carotid artery imaging using ultrasound (US) and magnetic resonance imaging (MRI) will be discussed, followed by the objectives and outline of this thesis.

\section{Ischemic stroke}

Cerebral (arterial) ischemic stroke is defined as an episode of neurological dysfunction caused by focal cerebral infarction. ${ }^{2}$ The pathophysiological mechanism of ischemic stroke is a drop in blood supply below a critical level, thus depriving brain parenchyma of oxygen and nutrients causing irreversible neuronal damage. Focal ischemia often results from occlusion of a cerebral artery supplying a specific brain area. Amongst the various causes of cerebrovascular ischemia, $15-20 \%$ of strokes are caused by artery-to-artery embolization, called large-vessel disease. ${ }^{3,4}$ Any vessel between the heart and brain parenchyma can be a source of such emboli. In clinical practice, however, the carotid artery bifurcation is the most common site of atherosclerotic plaque development causing cerebrovascular symptoms.

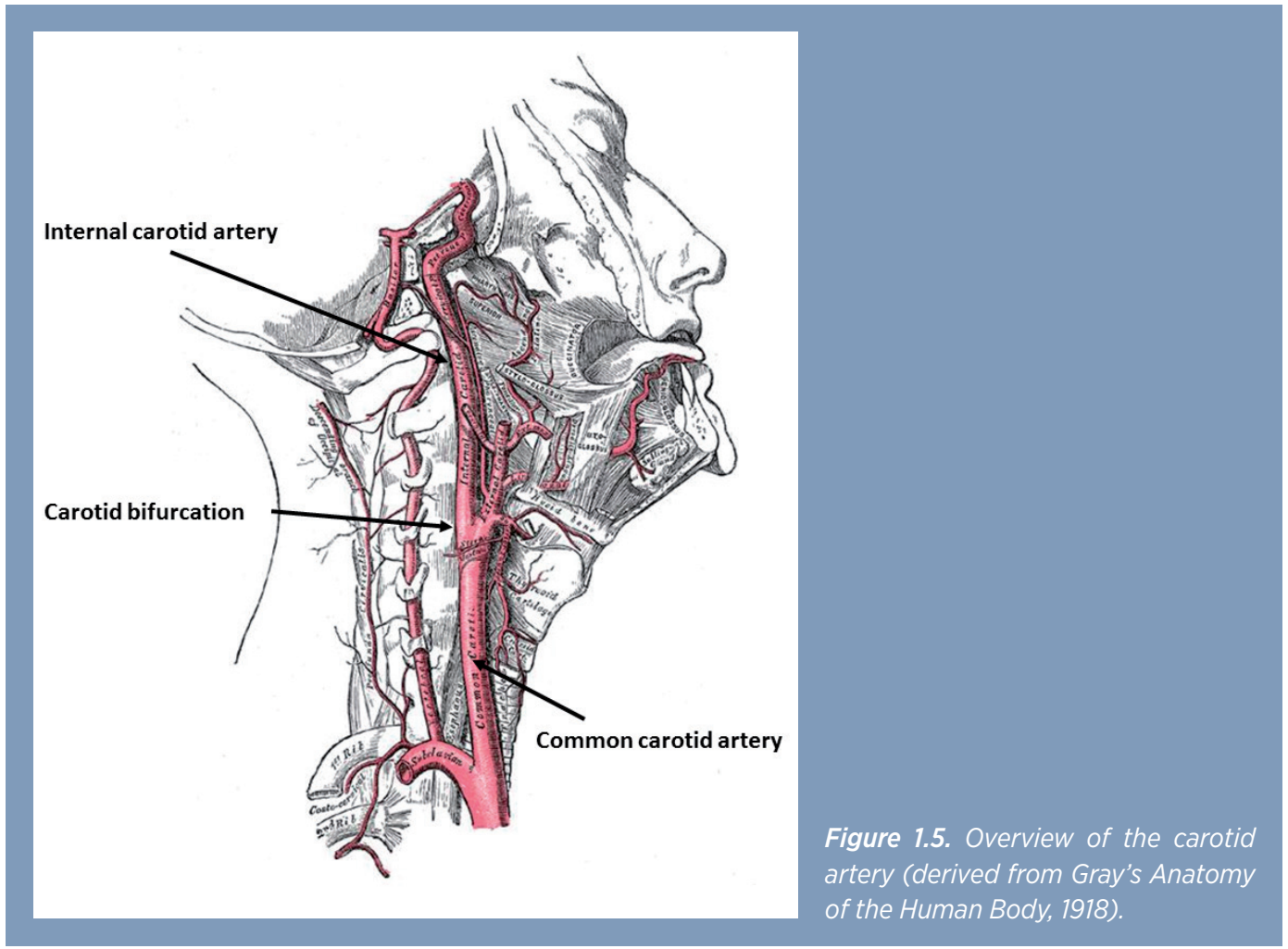


The carotid artery is the major vessel located in the anterolateral neck, just behind the sternocleidomastoid muscle (Figure 1.5). At the level of the larynx, the artery widens to form the carotid bulb, where the vessel bifurcates in the internal carotid artery (ICA) and external carotid artery (ECA). The part proximal of the carotid bifurcation is the common carotid artery (CCA). Whereas the ECA gives rise to several branches supplying the facial muscles, glands and skin of the face and neck, the ICA runs cranially without any branches before entering the skull and supplying the anterior two-thirds of the brain.

\section{Atherosclerosis: the vulnerable plaque}

The healthy arterial wall is composed of three layers (Figure 1.6). The inner layer is called the tunica intima. It consists of a single layer of endothelial cells, which is in contact with the circulating blood. Within the second layer, the tunica media, vascular smooth muscle cells and elastin fibers are located circumferentially to give the vessel its mechanical strength. The tunica adventitia is the outermost layer and consists of collagen which embeds the artery in its surroundings. Individual layers are separated from each other by elastic laminas.

Nowadays, atherosclerosis is considered a chronic, systemic inflammatory disease affecting large and medium-sized arteries. ${ }^{5-7}$ It results from the complex interplay of genetic profile and lifestyle factors. Early in life, plasma molecules and low density lipoprotein (LDL) cholesterol extravasate through the endothelial wall., 9 Oxidation of LDL triggers an inflammatory process that results in the accumulation of lipoproteins, macrophages and lymphocytes, forming a fatty streak within the intima., ${ }^{7}$ Lipid-loaded macrophages ultimately die by apoptosis and necrosis causing the soft lipid-rich, necrotic core (LRNC) within an atherosclerotic plaque to grow over time.

Plaque formation does not occur randomly. Under normal conditions, laminar blood flow in an artery exerts a steady pulsatile tangential drag force on the vascular wall called wall shear stress. ${ }^{10}$ This hemodynamic force has a major influence on endothelial hemostasis, including local vasodilatation, anti-inflammatory, antithrombotic and antihypertrophic effects through biochemical alternations and regulation of endothelial gene expression. Disturbed laminar flow, resulting from low or oscillatory wall shear stress, will induce endothelial dysfunction characterized by increased vascular wall permeability with subsequent influx of LDL cholesterol, leukocyte adherence and smooth muscle cell proliferation.11, 12 Atherosclerotic plaques typically develop at sites where laminar blood flow is disturbed, such as arterial bifurcations and focal widening of arteries. ${ }^{13}$, 14 The carotid artery bifurcation is specifically prone to atherosclerotic plaque development since at the level of the bifurcation, the carotid artery lumen also widens $10-20 \%{ }^{15,16}$

Over the course of decades, the atherosclerotic plaque steadily grows and protrudes into the lumen, causing focal narrowing of the lumen known as luminal stenosis. Not 


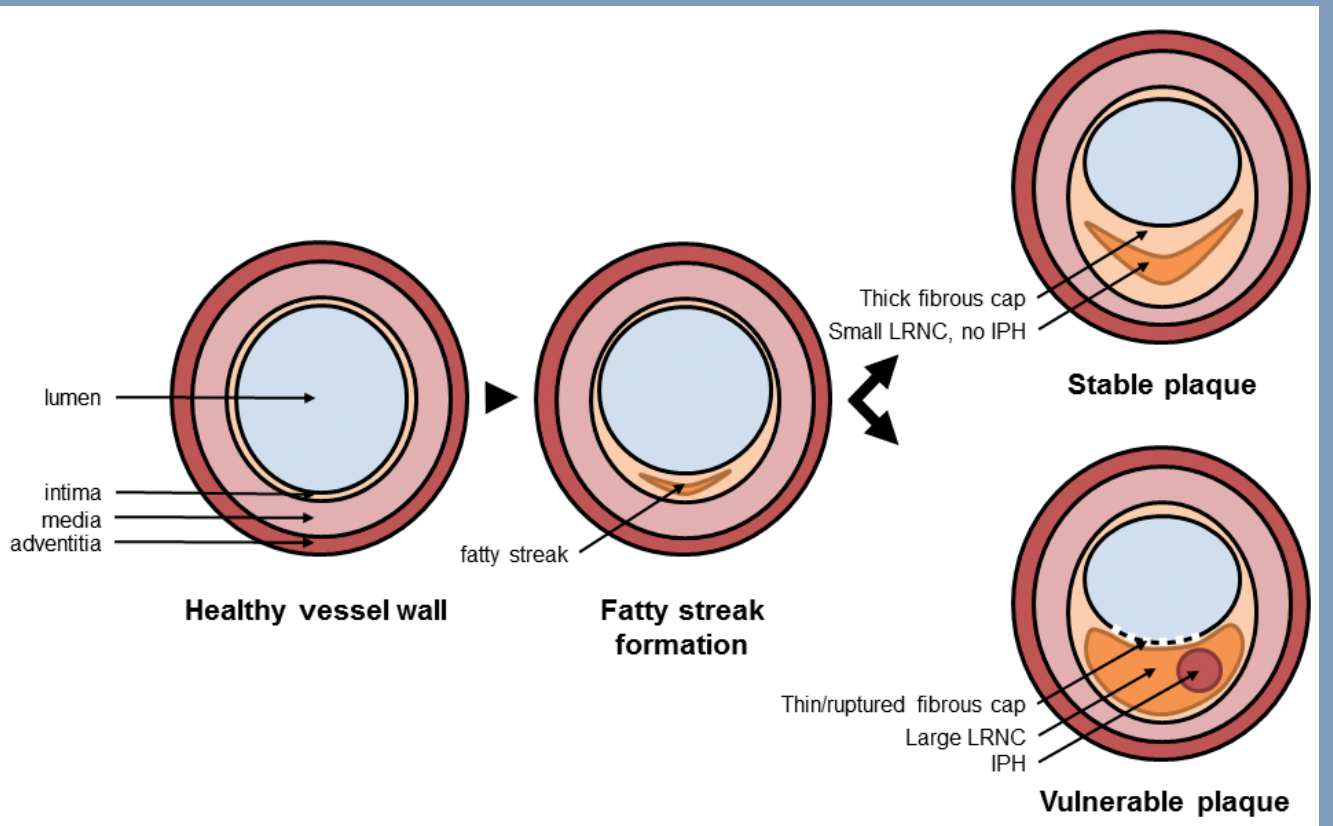

Figure 1.6. Cross-section of an artery: from healthy vessel wall to atherosclerotic plaque formation. Adapted from Ross e.a. ${ }^{5}$

all atherosclerotic plaques will lead to clinical symptoms. The risk of future stroke and the necessity for surgical intervention is currently appraised by the severity of luminal stenosis and whether or not there have been prior cerebrovascular events.17, 18 Of all patients having an asymptomatic carotid plaque with $60-99 \%$ stenosis, $93-94 \%$ will not suffer from cerebrovascular ischemia. ${ }^{19,20}$ How carotid plaques result in ischemic stroke has been matter of debate for many years. From the 1950-1960s to the mid-1980s, it was believed that the atherosclerotic plaque induces ischemic stroke through decreased cerebral perfusion distally of a hemodynamic significant stenosis. ${ }^{21,}{ }^{22}$ Nowadays, only a minority of ischemic strokes is attributed to cerebral hypoperfusion. ${ }^{23}$ Most cerebrovascular events are associated with less than $75 \%$ luminal stenosis, suggestive of an alternative cause. Pathological studies, first in coronary artery atherosclerosis, 24,25 and later also in carotid plaques ${ }^{26,27}$, demonstrated that most vascular ischemic events are caused by thrombus formation at the level of rupture or erosion of the fibrous cap overlying the atherosclerotic lesion. Angiographic studies confirmed that recently symptomatic carotid plaques frequently show signs of rupture. ${ }^{28}$ Due to cap rupture, the thrombogenic plaque content (i.e. lipid core, collagen, necrotic tissue) is exposed to the circulating blood causing luminal thrombosis, from which parts can be travel as thromboemboli to more distally located vessels. The majority of thromboemboli are composed of fibrin with 
platelet deposits, according to pathological examination of thrombus tissue recovered by endovascular stroke treatment. ${ }^{29}$

So if plaque rupture is the key factor for an indolent atherosclerotic plaque to suddenly become symptomatic, what are the main contributors to the plaque destabilization ultimately resulting in this rupture? And more importantly, can we identify a rupture-prone vulnerable plaque, before it will become symptomatic? Though the dynamic process of plaque destabilization is not completely understood, several factors are of importance: plaque composition, plaque inflammation and biomechanical stress on the plaque. First, the association between composition of atherosclerotic plaques and recent or future stroke has been identified through histological assessment ${ }^{27,30-33}$ and later confirmed by in vivo imaging ${ }^{34-37}$. Structural features of a vulnerable plaque are a large LRNC, a thin fibrous cap overlying the plaque, neovascularization, and intraplaque hemorrhage (IPH; Figure 1.6). IPH has been focus of attention in recent years, as detection of IPH on carotid plaque MRI is strongly associated with future stroke in several meta-analyses. ${ }^{38-40} \mathrm{IPH}$ may be caused by erythrocyte extravasation from leaky newly formed vessels within the plaque. ${ }^{41,42}$ Alternatively, several reports have shown a relation between small fissures in the fibrous cap and IPH, suggestive that IPH can also originate from hemorrhage from the lumen. ${ }^{25,43-45} \mathrm{IPH}$ results in plaque destabilization because it associated with rapid plaque expansion and can induce a strong inflammatory response due to the degradation of hemoglobin. ${ }^{42,46}$ The second feature of plaque vulnerability, inflammation, is not just important in fatty streak initiation but is a hallmark of plaque destabilization. Histology of ruptured plaques shows heavy infiltration of macrophages. ${ }^{27,47,48}$ These macrophages secrete proteolytic enzymes and cytokines which degrade the fibrous cap, induce apoptosis leading to enlargement of LRNC and stimulate angiogenesis which may trigger $\mathrm{IPH} .{ }^{42}$ In vivo quantification of plaque inflammation by means of Positron Emission Tomography (PET) imaging with ${ }^{18}$ F-fluoro-2-deoxy-D-glucose (FDG) ${ }^{49}, 50$ or ${ }^{18} \mathrm{~F}$-fluorocholin ${ }^{51}$ confirms the association with symptomatic plaque status.

Increased plaque biomechanical stress is the third feature of plaque vulnerability. It has received little attention up to recently although it was already acknowledged by Constantinides in the 1960s that a sudden increase in blood pressure could contribute to plaque rupture. ${ }^{52}$ In early systole, the arterial pressure wave is reflected at the proximal (upstream) plaque shoulder, resulting in a locally increased pulse pressure which is gradually decreased along the longitudinal axis of the plaque (Figure 1.7A, $p_{L}$ ). Additionally, the pulse pressure (i.e. the difference between systolic and diastolic blood pressure) applies a radial force on the arterial wall, distending the arterial diameter and compresses the plaque (Figure 1.7B, $p_{R}$ ). The net force (Figure 1.7C, $p_{\text {NET }}$ ) will deform the carotid plaque from early systole to diastole with every heartbeat, resulting in intraplaque strain. Plaque composition determines the extent and gradient of the deformation. The pressure gradient over the plaque is more likely to be a trigger for rupture than wall shear stress, since it can be a factor 100 larger than wall shear stress. ${ }^{53-55}$ Repetitive plaque 
deformation will ultimately lead to plaque rupture due to material fatigue or when the biomechanical stress exceeds the strength of the fibrous cap overlying the plaque. ${ }^{56,57}$

A

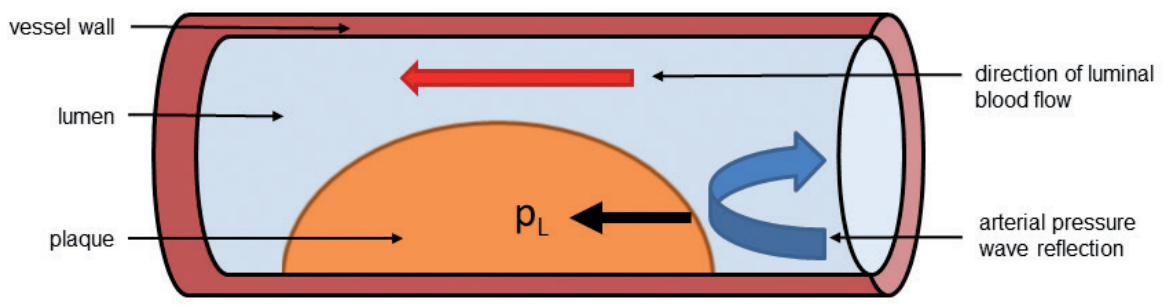

B

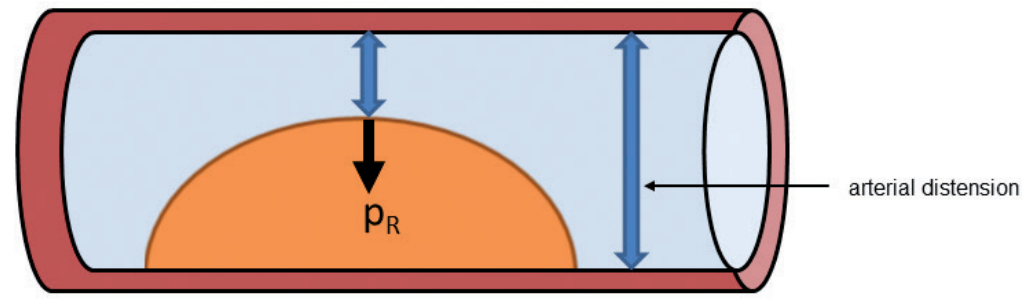

C

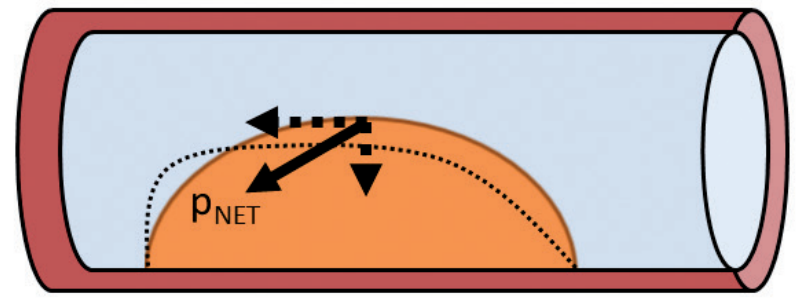

Figure 1.7. Longitudinal transection of an artery demonstrating the longitudinal (A), radial (B) and net force (C) working on a plaque, resulting in deformation from end-diastole (C: solid contour) to peak-systole (C: dashed contour).

Several studies using computational models suggest a role of plaque biomechanics in plaque rupture but also in the development of $\mathrm{IPH}^{58-61}$ Clinical research has shown that there is an association between pulse pressure and the presence of IPH and plaque rupture. ${ }^{62-64}$ Two studies demonstrated that the ulceration was found in the proximal plaque shoulder in $71 \%$ of angiographies and $75-86 \%$ of histological specimen, which can be considered as indirect evidence for the role of hemodynamics in plaque rupture. ${ }^{65}$, ${ }^{66}$ Additionally, inflammatory cells ${ }^{67-69}$ and vulnerable structural plaque features ${ }^{66,69}$ are also more often found in the proximal plaque shoulder. The pulsatile plaque deformation can be visualized by regular B-mode US..$^{70-72}$ More advanced ultrasound measurements can estimate strain within the vessel wall or within plaques. To date, few studies have correlated structural plaque features and intraplaque strain analysis in vivo, suggesting that vulnerable plaques may show reduced strain. ${ }^{73-75}$ 
As indicated above, there are various ways to study the features of plaque vulnerability. In this thesis, I will focus on US and MRI to study vessel wall and plaque composition and its functional behavior.

\section{Ultrasound imaging}

Diagnostic US imaging is the technique that enables noninvasive measurement and visualization of reflections from emitted ultrasound signals within the insonated tissue. ${ }^{76}$ The depth of the structures along a single ultrasound beam can be reconstructed from transit time between pulse emission and the registration of the reflections in the Amplitude mode (A-mode). Application of multiple ultrasound beams along the surface of a transducer can create a two-dimensional image, referred to as Brightness mode (B-mode). Based on the Doppler shift principle, movement (e.g. from passing blood flow) can be imaged and quantified in the Color mode. ${ }^{77}$ The combination of image modes is commonly referred to as duplex US.

In commercially available US systems, B-mode images undergo filtrations such as image thresholding and amplitude compression to reduce noise and improve image quality. As an alternative, unprocessed radio frequency (RF) data can also be used. ${ }^{78,79}$ Advantages of RF-based image analysis are 1) linear characteristics independent of gain settings; 2) data modification can be done offline without data loss; 3) temporal resolution can be increased up to 1000 frames per second, enabling simultaneous assessment of structural and functional vessel wall parameters; 4) improved depth resolution in motion analysis to allow assessment of tissue displacement. ${ }^{80}$ However, increasing temporal resolution will come at the cost of decreased spatial resolution.

For over 50 years, the effect of increasing luminal stenosis on local blood flow velocity is known. ${ }^{21}$ In the 1980s, measurement of blood flow velocity at the level of carotid plaque using Doppler measurements and later duplex US was correlated with the degree of stenosis established by conventional angiography. ${ }^{81,82}$ These studies resulted in cut-off values for peak-systolic and end-diastolic flow velocities to diagnose mild, moderate and severe stenosis (0-49\%, 50-69\% and 70-99\%, respectively). ${ }^{83}$ In a large meta-analysis, sensitivity and specificity of duplex US compared to conventional angiography has been shown to be very good for mild and severe stenosis ( $83 \%$ to $89 \%$ ), but sensitivity is poor (36\%) for moderate stenosis. ${ }^{84}$ Nowadays duplex US is the modality of choice for initial evaluation of carotid artery disease, as it is widely available, rapid, noninvasive, and can be performed at bedside at relatively low costs..$^{85-87}$

Besides the determination of degree of luminal stenosis by Doppler measurement of blood flow velocity, B-mode US can also be used to study vessel wall structure. The CCA is commonly used as a derivate to study atherosclerotic burden due to its location being easily assessable for US as opposed to the more challenging imaging of the ICA or carotid bifurcation. In the CCA, measurement of the intima-media thickness (IMT) was first 
described in $1986 .{ }^{88}$ The IMT is defined as the double-line patterns on the vessel wall depicted in the longitudinal direction and is usually averaged over a certain length (e.g. $10 \mathrm{~mm}$ ). ${ }^{89}$ Initially, IMT measurement was thought to truly reflect atherosclerotic disease and was considered a biomarker to predict future cardiovascular disease. ${ }^{90-93}$ IMT is however strongly associated with vascular risk factors and steadily increases with age..$^{94}$ Therefore, IMT and IMT progression add little to the risk prediction over traditional vascular risk factors. ${ }^{95-97}$

Alternatively, B-mode US can be used to depict plaques in the CCA, carotid bifurcation and ICA. Plaque is defined as 1) focal structure protruding into the lumen of at least $0.5 \mathrm{~mm} ; 2$ ) focal structure protruding into the lumen of $\geq 50 \%$ of the surrounding IMT value; or 3) focal structure with total wall thickness of $\geq 1.5 \mathrm{~mm} .{ }^{89}$ The severity of carotid atherosclerosis can be estimated by means of the carotid plaque score. ${ }^{98}$ Carotid plaque has been shown to better predict coronary and cerebrovascular events as compared to CCA-IMT. ${ }^{99-101}$

B-mode US can also be used to evaluate vulnerability using plaque echogenicity (i.e. the level of greyness assessed with the grey-scale median, GSM) as a measure to predict future risk of stroke in both symptomatic and asymptomatic patients..$^{102,103}$ Plaques with low GSM values (echolucent plaques) have been shown to correlate with the presence of LRNC and IPH on histological assessment, whereas plaques with high GSM (echointense plaque) are more likely to contain calcifications or large amounts of fibrous tissue..$^{104-107}$ As opposed to all other imaging techniques, US is the only modality that can directly depict and quantify the dynamic parameters, for example the change of the vessel wall diameter over the cardiac cycle (distension), with frequencies of $25-50 \mathrm{~Hz}$ (for regular B-mode US) up to a $1000 \mathrm{~Hz}$ (for RF-based US). US measurement of dynamic plaque behavior has been briefly mentioned in the previous paragraph.

\section{Magnetic resonance imaging}

$\mathrm{MRI}$ is a noninvasive imaging technique based on nuclear magnetic resonance of protons. Due to excellent soft tissue contrast, MRI of the atherosclerotic plaque is able to determine and quantify carotid plaque composition. From 2001 onwards, various studies have

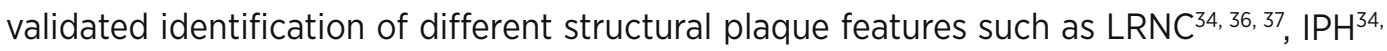
35,108 , thin and/or ruptured fibrous cap (TRFC) ${ }^{109,110}$ and neovascularization ${ }^{111}, 12$ on carotid MRI with histology as a reference. Combining multiple sequences provides additional information on the plaque components present. ${ }^{113} \mathrm{~A}$ review of 17 papers concludes that $\mathrm{MRI}$ analysis of plaque components shows good agreement with histology, though a lack of standardization hampered meta-analysis..$^{14}$ More importantly, MRI evidence of vulnerable plaque components is associated with an increased risk of cerebrovascular events in longitudinal follow-up studies in both symptomatic and asymptomatic patients. Three meta-analyses have summarized the literature for LRNC (hazard ratio (HR) 3.0; 95\% 
confidence interval (Cl): 1.5-6.0) ${ }^{39}$, TRFC (HR 5.9; 95\%-Cl 2.7-13.3) ${ }^{39}$ and IPH (HR between $4.6(95 \%-\mathrm{Cl} 2.9-7.2)$ and $12.2(95 \%-\mathrm{Cl} 5.5-27.1))^{38-40}$. A recent individual patient data metaanalysis has confirmed the value of detecting IPH. In 560 symptomatic patients, presence of IPH strongly increased the risk of ipsilateral stroke during a median follow-up duration of 16 months (HR 10.2; 95\%-Cl 4.6-22.5). ${ }^{115}$

\section{Objectives}

To summarize, the process of atherosclerotic plaque destabilization with subsequent plaque rupture resulting into clinical symptoms is only partly understood. Several noninvasive imaging techniques to study in vivo vessel wall and plaque structural and functional properties exist. This thesis aims to assess the structural and functional properties and its interplay using noninvasive imaging of the carotid artery wall and carotid artery plaques, as to gain more insight in the pathophysiology of atherosclerosis. The first part of this thesis will focus on structural and functional properties of the CCA, whereas the second part of this thesis will address these features in the ICA.

\section{Outline of this thesis}

Part I of the thesis focusses on subclinical atherosclerosis of the CCA. Chapter 2 compares B-mode and RF-based measurement methods to measure CCA-IMT. In addition, factors associated with a discrepancy between the two techniques are explored. Opposed to a smoothly enlarged IMT, a highly irregular vessel wall might be more indicative of atherosclerotic disease. In Chapter 3 we therefore study whether spatial IMTinhomogeneity is associated with the extent of more distally located atherosclerosis at the level of the carotid bifurcation.

In addition to structural vessel wall features, US can study functional vessel wall behavior. Distension is the change in vessel diameter over the cardiac cycle. Atherosclerosis is thought to be a generalized disease and although the CCA rarely contains significant luminal stenosis, the apparently normal CCA may undergo changes in distension as a result of arterial stiffening. Increased arterial stiffness is considered to result from cumulative vessel wall damage induced by vascular risk factors, for example hypertension. ${ }^{116}$ Chapter 4 will investigate the relation between distension along the CCA, spatial distension variation and the severity of more distally located atherosclerotic plaque. We theorize that plaque at the carotid bifurcation is associated with reduced vessel wall distension and increased spatial distension variation as part of the atherosclerotic process. In Chapter $\mathbf{5}$ we move from subclinical atherosclerotic changes to plaques located in the CCA. Due to its relative simple anatomy compared to the carotid bifurcation and ICA, we will use plaques in the CAA to evaluate a novel method to simultaneously study structural and functional plaque properties. 
In part II of this thesis, the focus is shifted from subclinical atherosclerosis of the CCA to atherosclerotic plaques at the carotid artery bifurcation, associated with recent ischemic stroke symptomatology. Currently, most acute stroke patients undergo duplex US of their carotid arteries to determine the presence and extent of carotid bifurcation atherosclerotic plaque. Duplex US uses stringent criteria of peak-systolic and enddiastolic flow velocities to establish degree of stenosis. ${ }^{82}$ However, blood flow velocity is inversely correlated with increasing age. ${ }^{117-119}$ In Chapter 6 we hypothesize that ageing may affect the accuracy of duplex ultrasound to determine the degree of carotid artery bifurcation stenosis.

Plaque biomechanical behavior has recently developed attention, since it may be an important but unrecognized hallmark of plaque vulnerability. However, since the use of advanced RF-based technique will not be widely available and requires sophisticated analyzing techniques, we propose an alternative and straightforward method of studying the influence of hemodynamic forces on carotid artery bifurcation plaques using routine B-mode ultrasonography. Simultaneous measurement of structural plaque features and of diastolic-to-systolic changes in carotid bifurcation plaque dimensions is examined in Chapter 7. We will evaluate the intra- and interobserver agreement and precision. Also, we aim to correlate these plaque features to plaque histopathology.

Carotid plaque MRI has been shown to correlate well with histology and several plaque components on carotid MRI are associated with an increased risk of future cerebrovascular events. Currently, carotid plaque MRI is only performed in specialized centers since and comprehensive MRI examination requires dedicated coils and expertise to interpret the images. In Chapter 8 we explore whether combining clinical features and routine B-mode US can be used to select patient with a high probability of having IPH on carotid MRI, thus being able to select patients eligible for carotid artery MRI.

Chapter 9 discusses the results of the research presented in this thesis. Moreover, it includes possible implications for clinical practice and future research. Research valorization as well as the English and Dutch summary of this thesis are presented in Chapter 10. 


\section{References}

1. Kwee RM, Truijman MTB, van Oostenbrugge RJ, Mess WH, Prins MH, Franke CL, et al. Longitudinal MRI study on the natural history of carotid artery plaques in symptomatic patients. PloS one. 2012;7:e42472

2. Sacco RL, Kasner SE, Broderick JP, Caplan LR, Connors JJ, Culebras A, et al. An updated definition of stroke for the 21st century: A statement for healthcare professionals from the American Heart Association/American stroke association. Stroke. 2013;44:2064-2089

3. Greco G, Egorova NN, Moskowitz AJ, Gelijns AC, Kent KC, Manganaro AJ, et al. A model for predicting the risk of carotid artery disease. Annals of surgery. 2013;257:1168-1173

4. Kolominsky-Rabas PL, Weber M, Gefeller O, Neundoerfer B, Heuschmann PU. Epidemiology of ischemic stroke subtypes according to TOAST criteria: Incidence, recurrence, and long-term survival in ischemic stroke subtypes: A population-based study. Stroke. 2001;32:2735-2740

5. Ross R. Atherosclerosis: An inflammatory disease. The New England journal of medicine. 1999;340:115-126

6. Falk E, Nakano M, Bentzon JF, Finn AV, Virmani R. Update on acute coronary syndromes: The pathologists' view. European heart journal. 2013;34:719-728

7. Falk E. Pathogenesis of atherosclerosis. Journal of the American College of Cardiology. 2006;47:C7-12

8. Kiechl S, Willeit J. The natural course of atherosclerosis. Part I: Incidence and progression. Arteriosclerosis, thrombosis, and vascular biology. 1999;19:1484-1490

9. Usman A, Ribatti D, Sadat U, Gillard JH. From lipid retention to immune-mediate inflammation and associated angiogenesis in the pathogenesis of atherosclerosis. Journal of Atherosclerisus and Thrombosis. 2015;22:739-749

10. Reneman RS, Arts T, Hoeks AP. Wall shear stress--an important determinant of endothelial cell function and structure--in the arterial system in vivo. Discrepancies with theory. Journal of vascular research. 2006;43:251-269

11. Cunningham KS, Gotlieb Al. The role of shear stress in the pathogenesis of atherosclerosis. Laboratory investigation; a journal of technical methods and pathology. 2005;85:9-23

12. Davies PF. Hemodynamic shear stress and the endothelium in cardiovascular pathophysiology. Nature clinical practice. Cardiovascular medicine. 2009;6:16-26

13. Friedman MH, Deters OJ, Mark FF, Bargeron CB, Hutchins GM. Arterial geometry affects hemodynamics. A potential risk factor for atherosclerosis. Atherosclerosis. 1983;46:225-231

14. Morbiducci U, Kok AM, Kwak BR, Stone PH, Steinman DA, Wentzel JJ. Atherosclerosis at arterial bifurcations: Evidence for the role of haemodynamics and geometry. Thrombosis and haemostasis. 2016;115:484-492

15. Lee SW, Antiga L, Spence JD, Steinman DA. Geometry of the carotid bifurcation predicts its exposure to disturbed flow. Stroke. 2008;39:2341-2347

16. Bijari PB, Wasserman BA, Steinman DA. Carotid bifurcation geometry is an independent predictor of early wall thickening at the carotid bulb. Stroke. 2014;45:473-478

17. Rothwell PM, Eliasziw M, Gutnikov SA, Fox AJ, Taylor DW, Mayberg MR, et al. Analysis of pooled data from the randomised controlled trials of endarterectomy for symptomatic carotid stenosis. The Lancet. 2003;361:107-116

18. Abbott AL, Paraskevas KI, Kakkos SK, Golledge J, Eckstein HH, Diaz-Sandoval LJ, et al. Systematic 
review of guidelines for the management of asymptomatic and symptomatic carotid stenosis. Stroke. 2015;46:3288-3301

19. Naylor AR. Time to rethink management strategies in asymptomatic carotid artery disease. Nature Reviews Cardiology. 2011;9:116-124

20. Naylor AR, Schroeder TV, Sillesen H. Clinical and imaging features associated with an increased risk of late stroke in patients with asymptomatic carotid disease. European journal of vascular and endovascular surgery. 2014;48:633-640

21. May AG, DeWeese JA, Rob CG. Hemodynamic effects of arterial stenosis. Surgery. 1963;53:513

22. May AG, Van de Berg L, DeWeese JA, Rob CG. Critical arterial stenosis. Surgery. 1963;54:250-259

23. Klijn CJM, Kappelle LJ. Haemodynamic stroke: Clinical features, prognosis, and management. The Lancet Neurology. 2010;9:1008-1017

24. Virmani R, Kolodgie FD, Burke AP, Farb A, Schwartz SM. Lessons from sudden coronary death: A comprehensive morphological classification scheme for atherosclerotic lesions. Arteriosclerosis, thrombosis, and vascular biology. 2000;20:1262-1275

25. Constantinides P. Plaque fissures in human coronary thrombosis. Journal of Atherosclerosis Research. 1966;6:1-17

26. Golledge J, Greenhalgh RM, Davies AH. The symptomatic carotid plaque. Stroke. 2000;31:774-781

27. Redgrave JN, Lovett JK, Gallagher PJ, Rothwell PM. Histological assessment of 526 symptomatic carotid plaques in relation to the nature and timing of ischemic symptoms: The Oxford plaque study. Circulation. 2006;113:2320-2328

28. Lovett JK, Gallagher PJ, Hands LJ, Walton J, Rothwell PM. Histological correlates of carotid plaque surface morphology on lumen contrast imaging. Circulation. 2004;110:2190-2197

29. Marder VJ, Chute DJ, Starkman S, Abolian AM, Kidwell C, Liebeskind D, et al. Analysis of thrombi retrieved from cerebral arteries of patients with acute ischemic stroke. Stroke. 2006;37:2086-2093

30. Virmani R, Ladich ER, Burke AP, Kolodgie FD. Histopathology of carotid atherosclerotic disease. Neurosurgery. 2006;59:S219-227; discussion S213-213

31. Plasschaert H, Heeneman S, Daemen MJ. Progression in atherosclerosis: Histological features and pathophysiology of atherosclerotic lesions. Topics in magnetic resonance imaging. 2009;20:227-237

32. Naghavi M, Libby P, Falk E, Casscells SW, Litovsky S, Rumberger J, et al. From vulnerable plaque to vulnerable patient: A call for new definitions and risk assessment strategies: Part I. Circulation. 2003;108:1664-1672

33. Howard DP, van Lammeren GW, Rothwell PM, Redgrave JN, Moll FL, de Vries JP, et al. Symptomatic carotid atherosclerotic disease correlations between plaque composition and ipsilateral stroke risk. Stroke. 2015;46:182-189

34. Yuan C, Mitsumori LM, Ferguson MS, Polissar NL, Echelard D, Ortiz G, et al. In vivo accuracy of multispectral magnetic resonance imaging for identifying lipid-rich necrotic cores and intraplaque hemorrhage in advanced human carotid plaques. Circulation. 2001;104:2051-2056

35. Cappendijk VC, Cleutjens KB, Heeneman S, Schurink GW, Welten RJ, Kessels AG, et al. In vivo detection of hemorrhage in human atherosclerotic plaques with magnetic resonance imaging. Journal of magnetic resonance imaging. 2004;20:105-110

36. Cappendijk VC, Kessels AG, Heeneman S, Cleutjens KB, Schurink GW, Welten RJ, et al. Comparison of lipid-rich necrotic core size in symptomatic and asymptomatic carotid atherosclerotic plaque: Initial results. Journal of magnetic resonance imaging. 2008;27:1356-1361 
37. Saam T, Ferguson MS, Yarnykh VL, Takaya N, Xu D, Polissar NL, et al. Quantitative evaluation of carotid plaque composition by in vivo MRI. Arteriosclerosis, thrombosis, and vascular biology. 2005;25:234-239

38. Saam T, Hetterich H, Hoffmann V, Yuan C, Dichgans M, Poppert H, et al. Meta-analysis and systematic review of the predictive value of carotid plaque hemorrhage on cerebrovascular events by magnetic resonance imaging. Journal of the American College of Cardiology. 2013;62:1081-1091

39. Gupta A, Baradaran H, Schweitzer AD, Kamel H, Pandya A, Delgado D, et al. Carotid plaque MRI and stroke risk: A systematic review and meta-analysis. Stroke. 2013;44:3071-3077

40. Hosseini AA, Kandiyil N, Macsweeney ST, Altaf N, Auer DP. Carotid plaque hemorrhage on magnetic resonance imaging strongly predicts recurrent ischemia and stroke. Annals of neurology. 2013;73:774-784

41. Michel JB, Virmani R, Arbustini E, Pasterkamp G. Intraplaque haemorrhages as the trigger of plaque vulnerability. European heart journal. 2011;32:1977-1985, 1985a, 1985b, 1985c

42. Virmani R, Kolodgie FD, Burke AP, Finn AV, Gold HK, Tulenko TN, et al. Atherosclerotic plaque progression and vulnerability to rupture: Angiogenesis as a source of intraplaque hemorrhage. Arteriosclerosis, thrombosis, and vascular biology. 2005;25:2054-2061

43. Daemen MJ, Ferguson MS, Gijsen FJ, Hippe DS, Kooi ME, Demarco K, et al. Carotid plaque fissure: An underestimated source of intraplaque hemorrhage. Atherosclerosis. 2016;254:102-108

44. Kolodgie FD, Gold HK, Burke AP, Fowler DR, Kruth HS, Weber DK, et al. Intraplaque hemorrhage and progression of coronary atheroma. The New England journal of medicine. 2003;349:2316-2352

45. Daemen MJ, Kooi ME. Intraplaque hemorrhage as a stimulator of episodic growth of advanced, but nonsymptomatic atherosclerotic lesions bridging the gap. JACC. Cardiovascular imaging. 2009;2:1390-1392

46. Takaya N, Yuan C, Chu B, Saam T, Polissar NL, Jarvik GP, et al. Presence of intraplaque hemorrhage stimulates progression of carotid atherosclerotic plaques: $\mathrm{A}$ high-resolution magnetic resonance imaging study. Circulation. 2005;111:2768-2775

47. Figueroa AL, Subramanian SS, Cury RC, Truong QA, Gardecki JA, Tearney GJ, et al. Distribution of inflammation within carotid atherosclerotic plaques with high-risk morphological features: A comparison between positron emission tomography activity, plaque morphology, and histopathology. Circulation cardiovascular imaging. 2012;5:69-77

48. van der Wal AC, Becker AE, van der Loos CM, Das PK. Site of intimal rupture or erosion of thrombosed coronary atherosclerotic plaques is characterized by an inflammatory process irrespective of the dominant plaque morphology. Circulation. 1994;89:36-44

49. Kwee RM, Truijman MT, Mess WH, Teule GJ, ter Berg JW, Franke CL, et al. Potential of integrated [18F] fluorodeoxyglucose positron-emission tomography/CT in identifying vulnerable carotid plaques. American journal of neuroradiology. 2011;32:950-954

50. Muller HF, Viaccoz A, Fisch L, Bonvin C, Lovblad KO, Ratib O, et al. ${ }^{18}$ FDG-PET-CT: An imaging biomarker of high-risk carotid plaques. Correlation to symptoms and microembolic signals. Stroke. 2014;45:3561-3566

51. Voo S, Kwee RM, Sluimer JC, Schreuder FH, Wierts R, Bauwens M, et al. Imaging intraplaque inflammation in carotid atherosclerosis with 18F-fluorocholine positron emission tomographycomputed tomography: Prospective study on vulnerable atheroma with immunohistochemical validation. Circulation cardiovascular imaging. 2016;9:pii: e004467 
52. Constantinides P, Chakravarti RN. Rabbit arterial thrombosis production by systemic procedures. Archives of Pathology. 1961;72:197-208

53. Li ZY, Taviani V, Tang T, Sadat U, Young V, Patterson A, et al. The mechanical triggers of plaque rupture: Shear stress vs pressure gradient. The British journal of radiology. 2009;82 Spec No 1:S39-45

54. Hoeks AP, Reesink KD, Hermeling E, Reneman RS. Local blood pressure rather than shear stress should be blamed for plaque rupture. Journal of the American College of Cardiology. 2008;52:11071108; author reply 1108-1109

55. Li ZY, Gillard JH. Plaque rupture: Plaque stress, shear stress, and pressure drop. Journal of the American College of Cardiology. 2008;52:499-500

56. Hoeks AP, Kooi ME. Wear and tear. JACC. Cardiovascular imaging. 2011;4:478-480

57. Pei X, Wu B, Tang TY, Gillard JH, Li ZY. Fatigue crack growth under pulsatile pressure and plaque rupture. JACC. Cardiovascular imaging. 2014;7:738-740

58. Huang X, Teng Z, Canton G, Ferguson M, Yuan C, Tang D. Intraplaque hemorrhage is associated with higher structural stresses in human atherosclerotic plaques: An in vivo MRI-based 3D fluid-structure interaction study. Biomedical Engineering Online. 2010;9:86

59. Teng Z, He J, Degnan AJ, Chen S, Sadat U, Bahaei NS, et al. Critical mechanical conditions around neovessels in carotid atherosclerotic plaque may promote intraplaque hemorrhage. Atherosclerosis. 2012;223:321-326

60. Cardoso L, Weinbaum S. Changing views of the biomechanics of vulnerable plaque rupture: $A$ review. Annals of biomedical engineering. 2014;42:415-431

61. Holzapfel GA, Mulvihill JJ, Cunnane EM, Walsh MT. Computational approaches for analyzing the mechanics of atherosclerotic plaques: A review. Journal of biomechanics. 2014;47:859-869

62. Selwaness M, van den Bouwhuijsen QJ, Verwoert GC, Dehghan A, Mattace-Raso FU, Vernooij M, et al. Blood pressure parameters and carotid intraplaque hemorrhage as measured by magnetic resonance imaging: The Rotterdam study. Hypertension. 2013;61:76-81

63. Sun J, Canton G, Balu N, Hippe DS, Xu D, Liu J, et al. Blood pressure is a major modifiable risk factor implicated in pathogenesis of intraplaque hemorrhage: An in vivo magnetic resonance imaging study. Arteriosclerosis, thrombosis, and vascular biology. 2016;36:743-749

64. Lovett JK, Howard SC, Rothwell PM. Pulse pressure is independently associated with carotid plaque ulceration. Journal of hypertension. 2003;21:1669-1676

65. Lovett JK, Rothwell PM. Site of carotid plaque ulceration in relation to direction of blood flow: An angiographic and pathological study. Cerebrovascular diseases. 2003;16:369-375

66. Cicha I, Wörner A, Urschel K, Beronov K, Goppelt-Struebe M, Verhoeven E, et al. Carotid plaque vulnerability: A positive feedback between hemodynamic and biochemical mechanisms. Stroke. 2011;42:3502-3510

67. Dirksen MT, van der Wal AC, van den Berg FM, van der Loos CM, Becker AE. Distribution of inflammatory cells in atherosclerotic plaques relates to the direction of flow. Circulation. 1998;98:2000-2003

68. Yilmaz A, Lipfert B, Cicha I, Schubert K, Klein M, Raithel D, et al. Accumulation of immune cells and high expression of chemokines/chemokine receptors in the upstream shoulder of atherosclerotic carotid plaques. Experimental and molecular pathology. 2007;82:245-255

69. Fagerberg B, Ryndel M, Kjelldahl J, Akyurek LM, Rosengren L, Karlstrom L, et al. Differences in lesion severity and cellular composition between in vivo assessed upstream and downstream sides of 
human symptomatic carotid atherosclerotic plaques. Journal of vascular research. 2010;47:221-230

70. Bang J, Dahl T, Bruinsma A, Kaspersen JH, Nagelhus Hernes TA, Myhre HO. A new method for analysis of motion of carotid plaques from rf ultrasound images. Ultrasound in medicine \& biology. 2003;29:967-976

71. Dahl T, Bang J, Ushakova A, Lydersen S, Myhre HO. Parameters describing motion in carotid artery plaques from ultrasound examination: A reproducibility study. Ultrasound in medicine \& biology. 2004;30:1133-1143

72. Meairs S, Hennerici M. Four-dimensional ultrasonographic characterization of plaque surface motion in patients with symptomatic and asymptomatic carotid artery stenosis. Stroke. 1999;30:1807-1813

73. Beaussier H, Naggara O, Calvet D, Joannides R, Guegan-Massardier E, Gerardin E, et al. Mechanical and structural characteristics of carotid plaques by combined analysis with echotracking system and MR imaging. JACC. Cardiovascular imaging. 2011;4:468-477

74. Hansen HH, de Borst GJ, Bots ML, Moll FL, Pasterkamp G, de Korte CL. Validation of noninvasive in vivo compound ultrasound strain imaging using histologic plaque vulnerability features. Stroke. 2016;47:2770-2775

75. Hansen HH, de Borst GJ, Bots ML, Moll FL, Pasterkamp G, de Korte CL. Compound ultrasound strain imaging for noninvasive detection of (fibro)atheromatous plaques: Histopathological validation in human carotid arteries. JACC Cardiovascular Imaging. 2016;9:1466-1467

76. Beach KW. Principles of ultrasonic imaging and instrumentarium. In: Nicolaides A, Beach KW, Kyriacou E, Pattichis CS, eds. Ultrasound and carotid bifurcation atherosclerosis. London: Springer -Verlag; 2012:67-96.

77. Flow and ultrasound basics. In: Valdueza JM, Schreiber SJ, Roehl J-E, Klingebiel R, eds. Neurosonology and neuroimaging of stroke. Stuttgart: Georg Thieme Verlag; 2008:2-12.

78. Brands PJ, Hoeks AP, Willigers J, Willekes C, Reneman RS. An integrated system for the non-invasive assessment of vessel wall and hemodynamic properties of large arteries by means of ultrasound. European Journal of Ultrasound. 1999;9:257-266

79. Hoeks AP, Willekes C, Boutouyrie P, Brands PJ, Willigers JM, Reneman RS. Automated detection of local artery wall thickness based on M-line signal processing. Ultrasound in medicine \& biology. 1997;23:1017-1023

80. Brands PJ, Hoeks AP, Ledoux LA, Reneman RS. A radio frequency domain complex cross-correlation model to estimate blood flow velocity and tissue motion by means of ultrasound. Ultrasound in medicine \& biology. 1997;23:911-920

81. Wolverson MK, Heiberg E, Sundaram M, Tantanasirviongse S, Shields JB. Carotid atherosclerosis: High-resolution real-time sonography correlated with angiography. American journal of roentgenology. 1983;140:355-361

82. Alexandrov AV, Brodie DS, McLean A, Hamilton P, Murphy J, Burns PN. Correlation of peak systolic velocity and angiographic measurement of carotid stenosis revisited. Stroke. 1997;28:339-342

83. Grant EG, Benson CB, Moneta GL, Alexandrov AV, Baker JD, Bluth El, et al. Carotid artery stenosis: Gray-scale and doppler US diagnosis--society of radiologists in ultrasound consensus conference. Radiology. 2003;229:340-346

84. Wardlaw JM, Chappell FM, Best JJK, Wartolowska K, Berry E. Non-invasive imaging compared with intra-arterial angiography in the diagnosis of symptomatic carotid stenosis: A meta-analysis. The Lancet. 2006;367:1503-1512 
85. Long A, Lepoutre A, Corbillon E, Branchereau A, Kretz JG. Modalities of preoperative imaging of the internal carotid artery used in France. Annals of vascular surgery. 2002;16:261-265

86. Osarumwense D, Pararajasingam R, Wilson P, Abraham J, Walker SR. Carotid artery imaging in the United Kingdom: A postal questionnaire of current practice. Vascular. 2005;13:173-177

87. Kwee RM, van Oostenbrugge RJ, Hofstra L, Teule GJ, van Engelshoven JM, Mess WH, et al. Identifying vulnerable carotid plaques by noninvasive imaging. Neurology. 2008;70:2401-2409

88. Pignoli P, Tremoli E, Poli A, Oreste P, Paoletti R. Intimal plus medial thickness of the arterial wall: A direct measurement with ultrasound imaging. Circulation. 1986;74:1399-1406

89. Touboul PJ, Hennerici MG, Meairs S, Adams H, Amarenco P, Bornstein N, et al. Mannheim carotid intima-media thickness and plaque consensus (2004-2006-2011). An update on behalf of the advisory board of the 3rd, 4th and 5th watching the risk symposia, at the 13th, 15th and 20th European Stroke Conferences, Mannheim, Germany, 2004, Brussels, Belgium, 2006, and Hamburg, Germany, 2011. Cerebrovascular diseases. 2012;34:290-296

90. Bots ML, Hoes AW, Koudstaal PJ, Hofman A, Grobbee DE. Common carotid intima-media thickness and risk of stroke and myocardial infarction: The Rotterdam study. Circulation. 1997;96:1432-1437

91. O'Leary DH, Polak JF, Kronmal RA, Manolio TA, Burke GL, Wolfson SKJ. Carotid-artery intima and media thickness as a risk factor for myocardial infarction and stroke in older adults. Cardiovascular health study collaborative research group. The New England journal of medicine. 1999;340:14-22

92. Touboul PJ, Elbaz A, Koller C, Lucas C, Adrai V, Chedru F, et al. Common carotid artery intima-media thickness and brain infarction: The etude du profil genetique de l'infarctus cerebral (genic) casecontrol study. The genic investigators. Circulation. 2000;102:313-318

93. Zureik M, Ducimetiere P, Touboul PJ, Courbon D, Bonithon-Kopp C, Berr C, et al. Common carotid intima-media thickness predicts occurrence of carotid atherosclerotic plaques: Longitudinal results from the aging vascular study (EVA) study. Arteriosclerosis, thrombosis, and vascular biology. 2000;20:1622-1629

94. Finn AV, Kolodgie FD, Virmani R. Correlation between carotid intimal/medial thickness and atherosclerosis: A point of view from pathology. Arteriosclerosis, thrombosis, and vascular biology. 2010;30:177-181

95. Spence JD. Measurement of carotid plaque burden. JAMA neurology. 2015;72:383-384

96. Bots ML, Hofman A, Grobbee DE. Increased common carotid intima-media thickness. Adaptive response or a reflection of atherosclerosis? Findings from the Rotterdam study. Stroke. 1997;28:24422447

97. Lorenz MW, Polak JF, Kavousi M, Mathiesen EB, Volzke H, Tuomainen TP, et al. Carotid intima-media thickness progression to predict cardiovascular events in the general population (the PROG-IMT collaborative project): A meta-analysis of individual participant data. Lancet. 2012;379:2053-2062

98. Spence JD, Eliasziw M, DiCicco M, Hackam DG, Galil R, Lohmann T. Carotid plaque area: A tool for targeting and evaluating vascular preventive therapy. Stroke. 2002;33:2916-2922

99. Mathiesen EB, Johnsen SH, Wilsgaard T, Bonaa KH, Lochen ML, Njolstad I. Carotid plaque area and intima-media thickness in prediction of first-ever ischemic stroke: A 10-year follow-up of 6584 men and women: The Tromso study. Stroke. 2011;42:972-978

100. Inaba Y, Chen JA, Bergmann SR. Carotid plaque, compared with carotid intima-media thickness, more accurately predicts coronary artery disease events: A meta-analysis. Atherosclerosis. 2012;220:128-133 
101. Johnsen SH, Mathiesen EB, Joakimsen O, Stensland E, Wilsgaard T, Lochen ML, et al. Carotid atherosclerosis is a stronger predictor of myocardial infarction in women than in men: A 6-year follow-up study of 6226 persons: The Tromso study. Stroke. 2007;38:2873-2880

102. Gupta A, Kesavabhotla K, Baradaran H, Kamel H, Pandya A, Giambrone AE, et al. Plaque echolucency and stroke risk in asymptomatic carotid stenosis: A systematic review and meta-analysis. Stroke. 2015;46:91-97

103. Jashari F, Ibrahimi P, Bajraktari G, Gronlund C, Wester P, Henein MY. Carotid plaque echogenicity predicts cerebrovascular symptoms: A systematic review and meta-analysis. European journal of neurology. 2016;23:1241-1247

104. El-Barghouty NM, Levine T, Ladva S, Flanagan A, Nicolaides A. Histological verification of computerised carotid plaque characterisation. European journal of vascular and endovascular surgery. 1996;11:414-416

105. Tegos TJ, Sohail M, Sabetai MM, Robless P, Akbar N, Pare G, et al. Echomorphologic and histopathologic characteristics of unstable carotid plaques. American journal of neuroradiology. 2000;21:1937-1944

106. Lal BK, Hobson RW, Pappas PJ, Kubicka R, Hameed M, Chakhtura EY, et al. Pixel distribution analysis of B-mode ultrasound scan images predicts histologic features of atherosclerotic carotid plaques. Journal of vascular surgery. 2002;35:1210-1217

107. Gronholdt MLM, Nordestgaard BG, Schroeder TV, Vorstrup S, Sillesen H. Ultrasonic echolucent carotid plaques predict future strokes. Circulation. 2001;104:68-73

108. Kampschulte A, Ferguson MS, Kerwin WS, Polissar NL, Chu B, Saam T, et al. Differentiation of intraplaque versus juxtaluminal hemorrhage/thrombus in advanced human carotid atherosclerotic lesions by in vivo magnetic resonance imaging. Circulation. 2004;110:3239-3244

109. Cai J, Hatsukami TS, Ferguson MS, Kerwin WS, Saam T, Chu B, et al. In vivo quantitative measurement of intact fibrous cap and lipid-rich necrotic core size in atherosclerotic carotid plaque: Comparison of high-resolution, contrast-enhanced magnetic resonance imaging and histology. Circulation. 2005;112:3437-3444

110. Kwee RM, van Engelshoven JM, Mess WH, ter Berg JW, Schreuder FH, Franke CL, et al. Reproducibility of fibrous cap status assessment of carotid artery plaques by contrast-enhanced MRI. Stroke. 2009;40:3017-3021

111. Gaens ME, Backes WH, Rozel S, Lipperts M, Sanders SN, Jaspers K, et al. Dynamic contrast-enhanced MR imaging of carotid atherosclerotic plaque: Model selection, reproducibility, and validation. Radiology. 2013;266:271-279

112. van Hoof RH, Hermeling E, Truijman MT, van Oostenbrugge RJ, Daemen JW, van der Geest RJ, et al. Phase-based vascular input function: Improved quantitative DEC-MRI of atherosclerotic plaques. Medical physics. 2015;42:4619

113. Cappendijk VC, Cleutjens KB, Kessels AG, Heeneman S, Schurink GW, Welten RJ, et al. Assessment of human atherosclerotic carotid plaque components with multisequence MR imaging: Initial experience. Radiology. 2005;234:487-492

114. den Hartog AG, Bovens SM, Koning W, Hendrikse J, Luijten PR, Moll FL, et al. Current status of clinical magnetic resonance imaging for plaque characterisation in patients with carotid artery stenosis. European journal of vascular and endovascular surgery. 2013;45:7-21

115. Schindler A, Schinner R, Altaf N, Hosseini AA, Simpson RJ, Esposito-Bauer L, et al. Prediction of 
stroke risk by detection of hemorrhage in carotid plaques: Meta-analysis of individual patient data. JACC Cardiovasc Imaging. 2020;13:395-406

116. Reference Values for Arterial Stiffness' Collaboration. Determinants of pulse wave velocity in healthy people and in the presence of cardiovascular risk factors: 'Establishing normal and reference values'. European Journal Heart. 2010;31:2338-2350

117. Müller M, Schimrigk K. A comparative assessment of cerebral haemodynamics in the basilar artery and carotid territory by transcranial doppler sonography in normal subjects. Ultrasound in medicine \& biology. 1994;20:677-687

118. Schmidt-Trucksäss A, Grathwohl D, Schmid A, Boragk R, Upmeier C, Keul J, et al. Structural, changes of the common carotid artery with age in male subjects. Arteriosclerosis, thrombosis, and vascular biology. 1999;19:1091-1097

119. Scheel P, Ruge C, Schoning M. Flow velocity and flow volume measurements in the extra-cranial carotid and vertebral arteries in healthy adults: Reference data and the effects of age. Ultrasound in medicine \& biology. 2000;26:1261-1266 




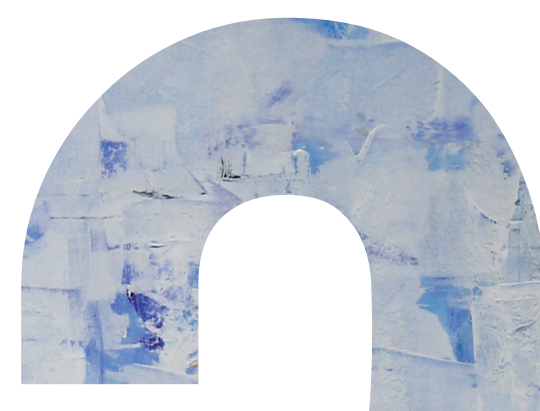

\section{MEASUREMENT OF COMMON CAROTID ARTERY INTIMA-MEDIA THICKNESS IN CLINICAL PRACTICE: COMPARISON OF B-MODE AND RF- BASED TECHNIQUE}

FHBM Schreuder, IM Graf, JM Hameleers, WH Mess,

APG Hoeks

Published in:

Ultraschall in der Medizin / European Journal of Ultrasound 2009; 30(5): 459-465 


\section{Abstract}

Purpose: The common carotid artery intima-media thickness (CCA-IMT) is usually measured using B-mode ultrasound images. A different approach for CCA-IMT detection is based on radio frequency (RF) multiple M-line analysis.

Materials and Methods: The present study explores the relationship between B-mode and RF measurement of CCA-IMT, as well as the reproducibility of both methods in 136 patients recently diagnosed with cardiovascular disease. Within one session, repeated measurements were made in the distal CCA bilaterally, using the B-mode (averaged over $10 \mathrm{~mm}$ ) and RF technique (averaging $12 \mathrm{M}$-lines over $14 \mathrm{~mm}$ ).

Results: The two methods correlate well (Pearson $r=0.765$ ). The CCA-IMT values measured with B-mode and RF were $0.779 \pm 0.196 \mathrm{~mm}$ and $0.734 \pm 0.172 \mathrm{~mm}$, respectively. B-mode CCA-IMT is significantly larger than RF CCA-IMT (mean difference of $0.045 \mathrm{~mm}$, SEM 7.8 $\mu \mathrm{m} ; \mathrm{t}=5.82 ; p<0.001$ ). In the multivariate regression analysis, carotid artery stenosis, inhomogeneous IMT and diabetes mellitus were the main predictors of differences between B-mode and RF CCA-IMT. The intrapatient variation for B-mode and RF-based CCA-IMT is comparable $(0.05 \pm 0.04 \mathrm{~mm}$ and $0.07 \pm 0.05 \mathrm{~mm}$, respectively).

Conclusion: CCA-IMT values measured with RF and B-mode have similar reproducibility and exhibit acceptable correlation, but RF CCA-IMT is significantly smaller. The difference between both methods is mainly due to advanced atherosclerosis. Hence, both methods can be used reliably to measure CCA-IMT in clinical practice. 


\section{Introduction}

Measurement of the intima-media thickness in the distal common carotid artery (CCAIMT) is increasingly being used as an independent risk factor for the development of cardiovascular events due to atherosclerosis. ${ }^{1-3}$ The CCA-IMT can be measured directly from B-mode images by manual or (semi-) automatic detection of the double-line pattern on the far wall. ${ }^{4}$ These two lines represent the lumen-intima and media-adventitia interface. The CCA-IMT is calculated from the distance between the two interfaces averaged over a designated segment length, generally on the order of $10 \mathrm{~mm}$. One should realize that B-mode images, as they are displayed, have been subjected to various processing stages, e.g. image thresholding to reject low amplitude signals and amplitude compression to accommodate large echo signals.

A different approach to measuring CCA-IMT is the use of radio frequency (RF) multiple M-line analysis., ${ }^{5,6}$ The RF data is unprocessed and retains linear characteristics independent of the gain settings; they can be evaluated offline and modified without the loss of original data. Another advantage of RF-based measurement of CCA-IMT is the possibility to simultaneously derive dynamic vessel wall properties, such as distension, strain and pulse wave velocity. ${ }^{7}$ Dynamic vessel wall properties can be accurately calculated through increased temporal resolution (i.e. increased frame rate) forcing a reduction of spatial display resolution (lower density of echo lines per image). A loss of spatial detail might introduce dissimilarities between B-mode and RF CCAIMT. Nevertheless, the RF method has previously proven to correlate well with B-mode measurement of the CCA-IMT in young, healthy subjects. ${ }^{9,10}$ However, the two techniques have not been compared in patients with clinical cardiovascular disease with anticipated irregular vessel wall structure. Measurement artefacts also occur more frequently in elderly subjects because of the loose embedding of arteries, allowing translational movement during the cardiac cycle.

The present study explores the relation between B-mode and RF-based measurement of CCA-IMT in subjects with evident cardiovascular disease. Factors responsible for any difference between both techniques are studied. In addition, the analysis includes the variation in sequential measurements of the CCA-IMT using both B-mode and RF data techniques to establish the reproducibility of both methods. 


\section{Materials and methods}

\section{Study subjects}

The study cohort was comprised of 162 patients referred for duplex ultrasonography of the carotid arteries from April to August 2007 (Table 2.1). The research was approved by the Medical Ethics Committee of the Maastricht University. All subjects gave written informed consent prior to the regular duplex examination. A standardized questionnaire designed to identify cardiovascular risk factors and previous medical history was recorded. Cerebrovascular events were defined as transient $(<24 \mathrm{~h})$ or permanent neurological symptoms due to cerebral ischemia. Cardiovascular disease included all known diagnoses of myocardial infarction, angina pectoris, atrial fibrillation and peripheral artery disease. Tobacco use was specified as current smoker, former smoker or non-smoker. The number of packyears smoked throughout the subject's life was calculated. The brachial artery blood pressure was measured using a semi-automatic oscillometric device (Dinamap, Critikon, Tampa, Florida, USA) averaging 6 recordings over a 30-minute period. Hypertension was defined as a blood pressure $\geq 140 \mathrm{mmHg}$ systolic and/or $\geq 90 \mathrm{mmHg}$ diastolic and/or current use of antihypertensive medication. Fasting plasma glucose levels $\geq 7.0 \mathrm{mmol} / \mathrm{I}$ or current use of blood glucose-lowering drugs were considered to be diagnostic for diabetes mellitus. Hypercholesterolemia was defined as a fasting blood total cholesterol level $\geq 6.5 \mathrm{mmol} / \mathrm{I}$ and/or LDL cholesterol level $\geq 4.5 \mathrm{mmol} / \mathrm{I}$ and/or present use of lipidlowering drugs.

Table 2.1. Indication for duplex ultrasonography ( $N=136$ subjects).

\begin{tabular}{lc} 
Indication & N (\%) \\
Stroke & $58(42.6 \%)$ \\
\hline TIA & $31(22.8 \%)$ \\
\hline Ocular ischemia & $9(6.6 \%)$ \\
\hline Follow-up after carotid endarterectomy & $4(2.9 \%)$ \\
\hline Screening & $7(5.2 \%)$ \\
$-\quad$ Pre-operative cardiac surgery & $27(19.9 \%)$ \\
$-\quad$ Other
\end{tabular}

\section{Data acquisition protocol}

Ultrasonography was performed by a well-trained staff in a room specially designed for ultrasound examinations. The subjects were examined in supine position with their heads slightly tilted to the opposite side.

All subjects underwent carotid duplex ultrasonography using a color-coded duplex machine (SONOS 5500, Philips Medical Systems, Eindhoven, Holland) equipped with a 3-11 MHz linear array transducer. In the common carotid and internal carotid artery, local blood flow velocities were determined at at least six distinct locations. The degree of 
stenosis was calculated using highest peak-systolic velocity present, according to the criteria outlined by the Society of Radiologists in Ultrasound." The degree of stenosis is listed in Table 2.2.

B-mode measurement of CCA-IMT was carried out using the same ultrasound machine (SONOS 5500). The scanning frequency was $7.5 \mathrm{MHz}$. The gain settings were standardized at a level to assure optimal image contrast. A three lead ECG signal served as the time reference. The region of interest was the distal left and right CCA, approximately $1 \mathrm{~cm}$ proximal to the onset of carotid bifurcation (Figure 2.1a). Preferably, the distal CCA was imaged on the plane of the bifurcation from an anterolateral approach. The longitudinal vessel axis was kept perpendicular to the transducer surface to image the double-line IMT pattern as horizontally as possible to establish clear lumen-intima and media-adventitia interfaces. A loop of $2.5 \mathrm{~s}$ with a frame rate of $25 \mathrm{~Hz}$ was recorded. Optimal visibility of the intima-media transition was confirmed before the B-mode data was stored on optical disks for offline analysis. Measurements were performed twice for both carotid arteries. Between consecutive measurements, the transducer was removed from the skin and replaced again to guarantee that both measurements were performed independently.

RF-based measurement of CCA-IMT was carried out using an ultrasound device (Picus, Esaote Europe, Maastricht, the Netherlands) equipped with a $7.5 \mathrm{MHz}$ linear array transducer, incorporating specialized software for RF-based measurements. ${ }^{7}$ The region of interest was first visualized in standard B-mode to ascertain the optimal location and angle of insonation. Signal gain settings were individually modified to obtain optimal image contrast during the measurement. Next, the ultrasound device was switched to multiple $\mathrm{M}$-line mode. The setting employs a reduced number of echo lines (14 echo lines covering $16.2 \mathrm{~mm}$ ) at an increased frame rate of $733 \mathrm{~Hz}$. ${ }^{6}$ The ECG was recorded along with data acquisition. A data loop of 3 seconds was stored for offline analysis on the hard disk of the computer. The transducer was removed from the skin and replaced between two consecutive measurements. The total data acquisition time including regular duplex ultrasonography of the carotid system was 30-45 minutes.

\section{Offline B-mode measurement of CCA-IMT}

The stored data from B-mode CCA-IMT recordings was analyzed using semi-automatic edge-detection software (Qlab 1.0, Philips Medical Software). For each heartbeat, the image corresponding to the end-diastolic phase was identified using the R-top of the ECG. After selecting the region of interest, the software automatically detected the lumen-intima and media-adventitia interfaces on the far wall and calculated the CCA-IMT averaged over a length of $10 \mathrm{~mm}$. All tracings were checked manually by a single observer (FS). The stored loops covered a mean of 3 heartbeats (range 2-5). The CCA-IMT was calculated as the mean IMT over the available heartbeats within a single measurement. 

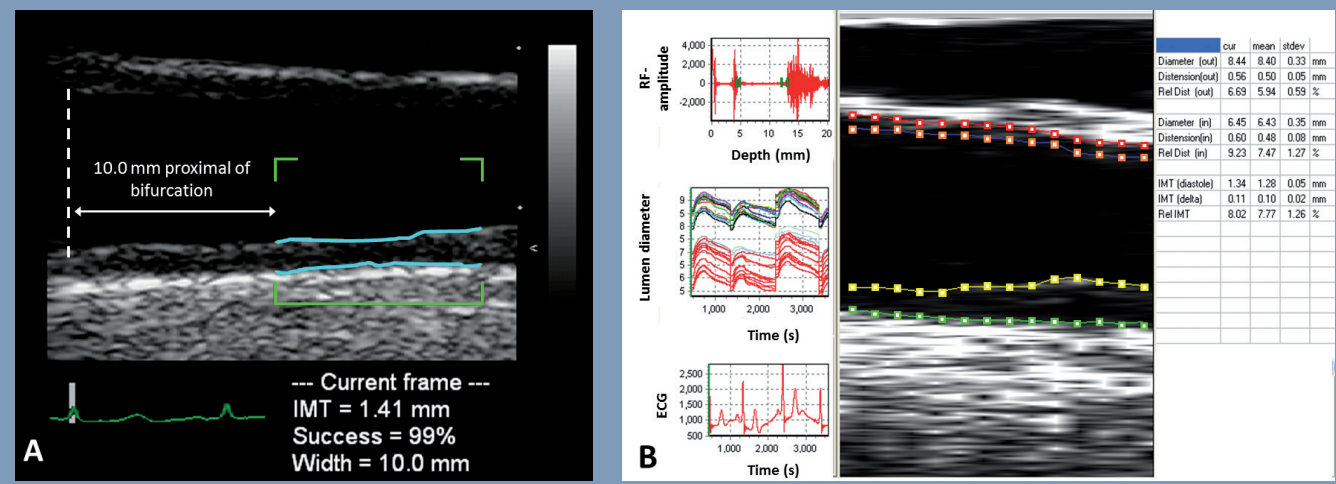

Figure 2.1. Panel a: Offline measurement of B-mode intima-media thickness (IMT) in the distal common carotid artery (CCA). Panel b: Offline RF-based measurement of intima-media thickness (IMT) in the distal common carotid artery (CCA) at the same carotid artery as a. The 14 echo-lines are represented by the 14 pairs of dots in the longitudinal direction. For echo-line number 13, the lumen-intima and media-adventitia transitions are marked in the RF-image (top left). The ECG trigger is shown on the left lower image. This image is a good example of an inhomogeneous distribution of IMT in the longitudinal axis of the far wall. A slight difference in observation plane results in a marked difference in IMT (B-mode IMT = $1.41 \mathrm{~mm}$ vs. RF-IMT $=1.28 \mathrm{~mm}$ ).

\section{Offline RF-based measurement of CCA-IMT}

The unprocessed RF data was recalled simultaneously with the ECG signals by a program developed by our research team. ${ }^{7}$ The algorithm automatically calculates the envelope of the RF data, which is displayed as a B-mode image, in parallel with a single RF line and ECG, each containing a marker of the position selection. The ECG signal was used as an automatic trigger to select end-diastolic values of CCA-IMT. A different observer (JH) manually marked the intima-media transition for each echo line in the B-mode images. For analysis, the two outer echo lines on either side were excluded. Therefore, the mean CCA-IMT was calculated over 12 echo lines covering $13.9 \mathrm{~mm}$ (Figure 2.1b). The final output of CCA-IMT was the average of all end-diastolic far wall CCA-IMT values over the recorded heartbeats (mean number of cardiac cycles: 3; range 2-6) within a single measurement. Local variations in vessel wall thickness (spatial CCA-IMT inhomogeneity) were established by the standard deviation (SD) of the spatial distribution of the CCA-IMT over the available 12 echo lines as the proportion of the mean CCA-IMT..$^{2}$ This method has already been used to detect local atherosclerotic lesions in end-stage renal disease patients..$^{13}$

\section{Feasibility of measurements}

We carried out both IMT measurements in a total of 162 subjects with CVD. B-mode CCAIMT images could not be used in 7 subjects due to a loss of data disk content. Additionally, 19 patients were excluded because of inferior quality of the RF data, mainly associated with poor visualization of the far wall as may happen in obese subjects. Measurement data from 136 patients (84\%) was ultimately included in the analysis. 


\section{Statistical analysis}

All statistical analyses were performed with the statistical software package SPSS for Windows (version 14.0). Data is presented as mean \pm SD. A scatterplot explored the relationship between B-mode and RF-based CCA-IMT. Pearson correlation coefficient ( $r$ ) was used to examine the level of correlation between B-mode and RF CCA-IMT. The concordance correlation coefficient (CCC) was computed to verify the agreement between the observed data and $45^{\circ}$ line of identity. ${ }^{14,15}$ The significance level was set at $p<0.05$. In addition, a Bland-Altman plot established the mean difference with a standard error of mean (SEM) and limits of agreement between the B- mode and RFbased CCA-IMT. ${ }^{16}$ A paired t-test determined whether any difference between B-mode and RF-based CCA-IMT was significant, together with 95\% confidence intervals $(\mathrm{Cl})$. The intraclass correlation coefficient (ICC) compared the variance among subjects to the total variance. ${ }^{15}$ Causes for significant differences between B-mode and RF CCAIMT were analyzed by the bivariate correlation between variables and the absolute difference in CCA-IMT. Variables with a significant influence on the difference between both techniques were explored using multiple linear regression analysis. Forced-entry introduction of these possible predictors established their individual contribution to the difference between B-mode and RF CCA-IMT.

Intrapatient variability was calculated for the relative difference between two consecutive measurements with the same technique for the entire population. The coefficients of variation (CV) assessed the intrapatient variation as the SD relative to the mean value. 


\section{Results}

The study population of 136 patients (mean age of $64.0 \pm 12.6$ years; $59.6 \%$ male) was at high risk of cardiovascular disease as reflected by the widespread presence of traditional risk factors (Table 2.2). Diabetes mellitus was recorded in 25 subjects (18.4\%), and 107 subjects of whom 22 subjects had not yet been treated were hypertensive (78.7\%). Sixtysix patients used lipid-lowering drugs. The cholesterol levels in an additional 13 subjects exceeded the limits. Most subjects (76.5\%) were classified as current smokers or had smoked in the past. The mean amount of packyears was 30.8. Previous cerebrovascular (26.5\%) and cardiovascular diseases (39.7\%) were commonly present.

B-mode and RF-based measurements of CCA-IMT were $0.779 \pm 0.196 \mathrm{~mm}$ (range 0.45 $1.60 \mathrm{~mm}$ ) and $0.734 \pm 0.172 \mathrm{~mm}$ (range $0.39-1.61 \mathrm{~mm}$ ), respectively. The mean CCA-IMT increased steadily with age at a rate of $6 \pm 1 \mu \mathrm{m} /$ year $(r=0.418 ; p<0.001)$. Overall, the intrapatient variation revealed no significant difference between the left and right carotid artery, allowing data pooling of both. The relationship between B-mode and RF-based CCA-IMT is illustrated in Figure 2.2. Linear regression analysis determined the relationship as: $\mathrm{B}$-mode CCA-IMT $=0.137+0.874{ }^{*} \mathrm{RF}$ CCA-IMT. The y-intercept is $0.137(95 \%-\mathrm{Cl}$ : 0.071 - 0.203; SE 0.034), being significantly different from $0(t=4.12, p<0.001)$. In addition, the slope is 0.874 (95\%-Cl: $0.788-0.960$; SE 0.045), being significantly different from 1 $(\mathrm{t}=19.39, p<0.001)$. Pearson $r$, ICC and CCC were $0.765,0.737$ and $0.736(96.2 \%$ of $r$ ), respectively.

Agreement between B-mode and RF-based measurement of CCA-IMT is shown in a BlandAltman scatterplot (Figure 2.3). The mean difference between B-mode and RF-based CCA-IMT was $0.045 \mathrm{~mm}$ (SEM $7.8 \mu \mathrm{m} ; 95 \%-\mathrm{Cl}: 0.029-0.060 \mathrm{~mm}$ ), which was significant in the paired $t$-test $(t=5.82 ; p<0.001)$. The limits of agreement in the Bland-Altman analysis ranged from $-0.207 \mathrm{~mm}$ to $0.297 \mathrm{~mm}$. When the ten major outliers (outside the limits of agreement) were excluded, the difference between the two methods decreased to 0.028 $\mathrm{mm}$ (SEM $5.9 \mu \mathrm{m} ; 95 \%-\mathrm{Cl}: 0.016-0.039 \mathrm{~mm}$ ), which however remained significant in the $\mathrm{t}$-test $(\mathrm{t}=4.77, p<0.001)$. Consequently, the limits of agreement improved from -0.159 to $0.214 \mathrm{~mm}$. The correlation between the two methods improved to $r=0.867$. 
Table 2.2. Demographic data of the 136 study subjects. ${ }^{a}$

\section{N (range or \%)}

Age (years)

$64.0 \pm 12.6(33-95)$

Sex (male)

$81(59.6 \%)$

\section{Tobacco use:}

- Current/former/non-smoker

46 (33.8\%) / 58 (42.7\%) / 32 (23.5\%)

- Packyears $30.8 \pm 19.7(3-115)$

\begin{tabular}{|c|c|}
\hline Diabetes mellitus ${ }^{\text {b }}$ & $25(18.4 \%)$ \\
\hline Hypertension ${ }^{c}$ & $107(78.8 \%)$ \\
\hline Hypercholesterolemia d & $79(58.1 \%)$ \\
\hline Previous cerebrovascular event & $36(26.5 \%)$ \\
\hline Previous cardiovascular event & $54(39.7 \%)$ \\
\hline $\operatorname{BMI}\left(\mathrm{kg} / \mathrm{m}^{2}\right)$ & $26.3 \pm 4.7(17-40)$ \\
\hline Systolic BP (mmHg) & $143 \pm 23(85-228)$ \\
\hline Diastolic BP (mmHg) & $80 \pm 12(45-123)$ \\
\hline Fasting glucose (mmol/l) & $6.2 \pm 1.8(3.5-14.9)$ \\
\hline \multicolumn{2}{|l|}{ Cholesterol } \\
\hline - $\quad$ Total cholesterol $(\mathrm{mmol} / \mathrm{l})$ & $5.3 \pm 1.2(2.6-9.0)$ \\
\hline - $\quad$ HDL cholesterol $(\mathrm{mmol} / \mathrm{l})$ & $1.2 \pm 0.4(0.6-2.5)$ \\
\hline - $\quad$ LDL cholesterol (mmol/l) & $3.4 \pm 1.0(1.5-7.2)$ \\
\hline \multicolumn{2}{|l|}{ Degree of carotid stenosis } \\
\hline - $\quad$ Bilateral $<50 \%$ & $106(77.9 \%)$ \\
\hline - $\quad$ Unilateral 50-70\% & $10(7.4 \%)$ \\
\hline - $\quad$ Bilateral 50-70\% & $1(0.7 \%)$ \\
\hline - Unilateral $>70 \%$ e & $18(13.3 \%)$ \\
\hline - $\quad$ Bilateral > 70\% e & $1(0.7 \%)$ \\
\hline
\end{tabular}

${ }^{a}$ Data is expressed as mean $\pm S D$, with ranges following in parenthesis. BMI, body mass index; $B P$, blood pressure.

${ }^{b}$ Defined as fasting glucose $\geq 7.0 \mathrm{mmol} / \mathrm{l}$ or present medication use.

c Defined as blood pressure $\geq 140 / 90 \mathrm{mmHg}$ or present medication use.

a Defined as total cholesterol $\geq 6.5 \mathrm{mmol} / \mathrm{l}$ or present medication use.

e Also included total occlusions. 


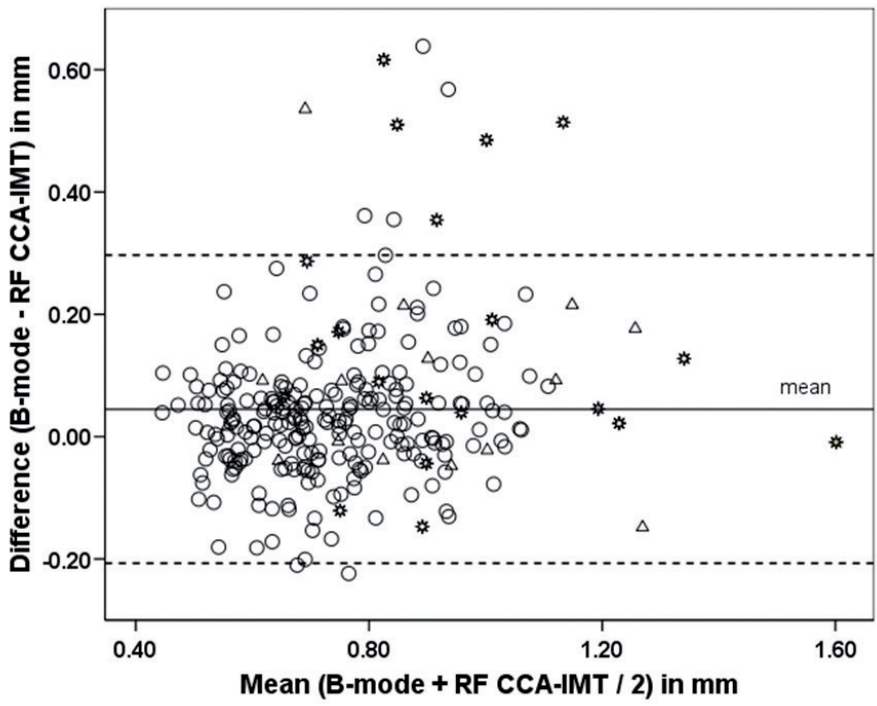

Figure 2.2. Association between B-mode measurement and RF-based measurement of common carotid artery intima-media thickness (CCA-IMT) in 136 subjects ( $n=272$ arteries). The solid line represents the regression line with its $95 \%-\mathrm{Cl}$. The dashed line represents the $45^{\circ}$ line of identity. The dots represent arteries with carotid stenosis $<50 \%(n=238)$, the triangles pertain to arteries with $50-70 \%$ stenosis $(n=$ 14) whereas the stars correspond to stenosis $>70 \%$ lumen reduction $(n=20)$.

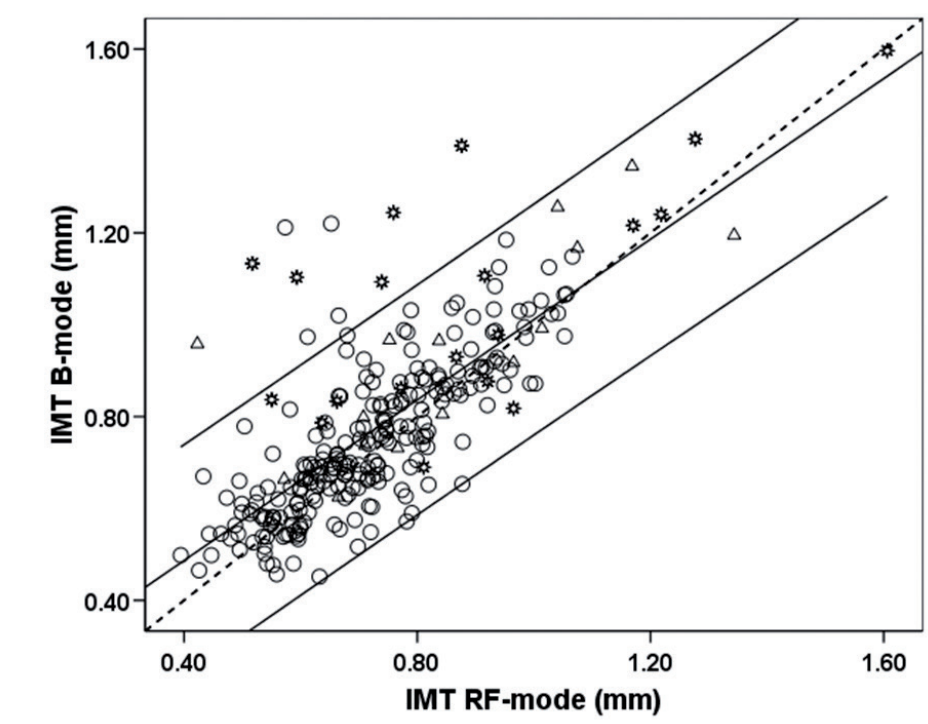

Figure 2.3. Agreement between B-mode measurement and RF-based measurement of common carotid artery intima-media thickness (CCA-IMT) in 136 subjects ( $n=272$ arteries). Lines are drawn for the mean difference and the 95\%-Cl. The dots represent arteries with carotid stenosis < 50\% $(n=238)$, the triangles pertain to arteries with 50 - 70\% stenosis $(n=14)$ whereas the stars correspond to stenosis $>70 \%$ lumen reduction $(n=20)$. 
In subjects lacking carotid plaques, the difference between both methods was significantly smaller when compared to subjects with plaques $(32.0 \pm 4.7 \mu \mathrm{m}$ and $49.1 \pm 9.7 \mu \mathrm{m}$, respectively). Upon bivariate analysis, CCA-IMT ( $r=0.188 ; p=0.002)$, IMT inhomogeneity $(r=0.166 ; p=0.006)$, increasing degree of stenosis of the ipsilateral carotid artery $\left(F_{3}=\right.$ $4.5 ; p=0.004)$, diabetes mellitus $\left(F_{1}=4.6 ; p=0.033\right)$ as well as male sex $\left(F_{1}=4.4 ; p=\right.$ 0.036 ) were found to correlate significantly with differences between B-mode and RFbased CCA-IMT (Table 2.3). These five variables were analyzed in forced-entry multiple regression analysis with a difference in CCA-IMT between both methods as a dependent variable. When included in a multiple regression model, only CCA-IMT inhomogeneity, degree of stenosis and diabetes mellitus were significant predictors of the occurrence of a difference between B-mode and RF-based CCA-IMT (Table 2.4; $F_{5,266}=8.3, p<0.001$ ).

Table 2.3. Variables correlated with a difference between CCA-IMT values measured with B-mode and RF in a bivariate correlation analysis ( $n=136$ subjects).

\begin{tabular}{|c|c|c|}
\hline & Statistical test ${ }^{a}$ & $p$-value \\
\hline CCA-IMT (mm) & $r=0.188$ & $0.002^{b}$ \\
\hline IMT inhomogeneity (\%) & $r=0.166$ & $0.006^{b}$ \\
\hline Age (years) & $r=0.006$ & 0.919 \\
\hline Sex (male/female) & $\mathrm{F}_{1}=4.4$ & $0.036^{b}$ \\
\hline Smoking (current/former/non-smoker) & $F_{2}=0.8$ & 0.449 \\
\hline Diabetes mellitus (yes vs. no) & $F_{1}=4.6$ & $0.033^{b}$ \\
\hline Hypertension (yes vs. no) & $F_{1}=1.3$ & 0.261 \\
\hline Hypercholesterolemia (yes vs. no) & $F_{1}=0.4$ & 0.506 \\
\hline Previous cerebrovascular event (yes vs. no) & $F_{1}=1.9$ & 0.167 \\
\hline Previous cardiovascular event (yes vs. no) & $F_{1}=0.3$ & 0.609 \\
\hline BMI $\left(\mathrm{kg} / \mathrm{m}^{2}\right)$ & $r=0.015$ & 0.805 \\
\hline Systolic BP (mmHg) & $r=0.053$ & 0.389 \\
\hline Diastolic BP (mmHg) & $r=-0.063$ & 0.309 \\
\hline Total cholesterol (mmol/l) & $r=0.089$ & 0.150 \\
\hline HDL/LDL cholesterol ratio & $r=-0.071$ & 0.258 \\
\hline $\begin{array}{l}\text { Ipsilateral stenosis degree }(<50 \%, 50-70 \%, 71-99 \% \text {, } \\
\text { occlusion) }\end{array}$ & $F_{3}=4.5$ & $0.004^{b}$ \\
\hline
\end{tabular}

a Pearson correlation coefficient $(r)$ is used for continuous variables, whereas one-way ANOVA $(F)$ is used for ordinal variables. ${ }^{b}$ Significant $(p<0.05)$. 
Table 2.4. Multiple regression coefficients responsible for differences between common carotid intima-media thickness (CCA-IMT) measured with B-mode and RF.

\begin{tabular}{lcccc} 
& \multicolumn{4}{c}{ Difference between B-mode and RF CCA-IMT } \\
& B & SE B & $\boldsymbol{\beta}$ & $\boldsymbol{p}$-value \\
\hline Constant & 0.049 & 0.003 & & $<0.001$ \\
\hline CCA-IMT & 0.070 & 0.036 & 0.12 & 0.052 \\
\hline Degree of stenosis & 0.033 & 0.009 & 0.21 & 0.001 \\
\hline CCA-IMT inhomogeneity (\%) & 0.004 & 0.002 & 0.16 & 0.009 \\
\hline Diabetes mellitus & 0.036 & 0.015 & 0.14 & 0.040 \\
\hline Male sex & 0.018 & 0.012 & 0.08 & 0.241 \\
\hline
\end{tabular}

${ }^{a} B$, non-standardized coefficient; SE, standard error; $\beta$, standardized coefficient.

Lastly, the reproducibility was explored. The B-mode and RF-based CCA-IMT intrapatient variation was $0.046 \pm 0.039 \mathrm{~mm}(5.9 \%$ of mean B-mode CCA-IMT) and $0.065 \pm 0.049$ $\mathrm{mm}$ (8.9\% of mean RF CCA-IMT), respectively. The CV for B-mode and RF CCA-IMT were $3.8 \%$ and $9.1 \%$, respectively. 


\section{Discussion}

In a population of subjects recently diagnosed with cardiovascular disease, mainly cerebrovascular ischemia, RF-based CCA-IMT correlates well with regularly used B-mode measurement $(r=0.765)$. Also, the regression line corresponds well with the identity line (i.e. excellent agreement between both methods) as expressed by the CCC ( $96.2 \%$ of $r$ ). Analysis of the reproducibility illustrated smaller variations between separate measurements in B-mode when compared to the RF method $(5.9 \%$ vs. $8.9 \%$ of mean IMT).

Previously, Willekes et al. demonstrated a strong correlation $(r=0.86)$ between offline RF and B-mode measurements of CCA-IMT in a small set of healthy young subjects $(n=29) .{ }^{9}$ Another study in 250 healthy subjects reported a slightly lower correlation coefficient $(r=0.808)$, yet still higher than in the current study..$^{10}$ However, both studies strictly included healthy subjects. Consequently, the mean CCA-IMT in those studies was considerably smaller than our results $\left(0.59 \mathrm{~mm}^{9}\right.$ and $0.66 \mathrm{~mm}^{10} \mathrm{vs} .0 .78 \mathrm{~mm}$ for B-mode measurements), which is most likely caused by the high prevalence of cardiovascular disease as well as higher mean age (64 years in the present study vs. 39 years ${ }^{9}$ and 50 years ${ }^{10}$.

Even though our study demonstrates a good correlation between B-mode and RF CCA-IMT, there is a significant difference of $45 \mu \mathrm{m}$ between the two methods. Theoretically, any difference between RF and B-mode CCA-IMT is caused by the various stages of image processing in the B-mode measurement. Recently, the influence of gain settings and dynamic range on the in-vitro detection of arterial diameter and IMT has been demonstrated. ${ }^{17}$ Our study group has recently confirmed these effects in vivo but these results have not yet been published. In concordance with our study, Willekes et al. also demonstrated a $31 \mu \mathrm{m}$ lower CCA-IMT when derived with the RF method compared to the B-mode technique. ${ }^{9}$ However, van Bortel et al. obtained a difference of $11 \mu \mathrm{m}$ in favor of the RF based measurements. ${ }^{10}$ Thus, it cannot be definitively concluded that RF measurements systematically result in a lower CCA-IMT compared to B-mode.

In the present study, limits of agreement are significantly larger $(-0.207 \mathrm{~mm}$ to $0.297 \mathrm{~mm}$ ) than reported previously $\left(-0.109 \mathrm{~mm}\right.$ to $0.171 \mathrm{~mm}^{9}$ and $-0.167 \mathrm{~mm}$ to 0.188 $\mathrm{mm}^{10}$ ). This finding may be caused by a dozen measurements with a very large difference between both methods (Figure 2.2). Upon exclusion of ten major discrepancies, the limits of agreement improved to be comparable with van Bortel without affecting the level of significance of the difference between the two methods. ${ }^{10}$ The major differences in both measures probably result from atherosclerotic vessel wall irregularities, introducing quite different IMTs for a slight difference in the observation (Figure 2.1). The vessel wall irregularities can be detected by measurement of the local IMT inhomogeneity. Indeed, our results confirm that spatial CCA-IMT inhomogeneity and distally located carotid artery stenosis are significant predictors of incidental differences between B-mode and RF CCA- 
IMT. Thus, the difference between B-mode and RF CCA-IMT was mainly caused by more advanced atherosclerosis. The intima-media thickness increases steadily with ageing, which has been thought to result from vascular adaptations to the loss of vessel wall elasticity. ${ }^{18}$ In our population, the annual rate of IMT increase was $6 \pm 1 \mu \mathrm{m}$ in agreement with the available literature. ${ }^{18-20}$ It is challenging to differentiate an age-related increase in CCA-IMT from an enlarged IMT due to vascular atherosclerotic disease. Hermans et al. recently showed that an inhomogeneous CCA-IMT correlates to the presence of cardiovascular disease. The measurement of vessel wall inhomogeneity might enhance the differentiation between the ageing process and definite atherosclerotic lesions. Hence, future IMT measurements should also include measurements of inhomogeneity since it has implications for the interpretation of results.

The reproducibility of the $\mathrm{B}$-mode measurement in the present study is in concordance with previous studies. ${ }^{21-24}$ An explanation for the higher intrapatient variation of RF measurements may be due to the fact that RF CCA-IMT is independently determined for the considered 12 echo lines, while B-mode uses smoothing algorithms to derive the spatial distribution of CCA-IMT. This is confirmed by the coefficient of variation (i. e. illustrating the variance of CCA-IMT within a single image), which is nearly 2.5 times larger for RF measurements (9.1 vs. 3.8\%). The eventual difference in reproducibility between both methods seems to be rather small $(46 \mu \mathrm{m}$ vs. $65 \mu \mathrm{m}$ ). In RF ultrasound devices it is possible to increase the number of echo lines as desired by the operator. Hence, the difference in reproducibility might be eliminated in clinical practice. The RF method has the additional advantage of supplying supplementary parameters, such as dynamic vessel wall characteristics. The combined evaluation of both dynamic and morphological parameters (mean value, irregularity) might provide more detailed insight into the individual atherosclerotic burden and may improve our understanding of atherogenesis as a whole.

The present study has certain limitations. First, RF measurements were excluded in approximately $10 \%$ of patients. This was mainly due to inferior visualization of the far wall, for instance in obese subjects, as well as in tortuous carotid arteries. In addition, B-mode CCA-IMT was averaged over $10 \mathrm{~mm}$, while RF CCA-IMT was averaged over 14 $\mathrm{mm}$. The difference in considered arterial wall length influences the standard error of the mean CCA-IMT estimate, but this discrepancy would favor CCA-IMT only with $20 \%$. Lastly, consecutive measurements were not standardized for measurement at the exact same location. This may have been a major cause of a difference between the two techniques. However, we believe that this is a better reflection of clinical practice, in which intersession measurements are performed. 


\section{Conclusion}

To conclude, the current study indicates that RF CCA-IMT is significantly lower compared to the established B-mode measurement. The degree of carotid artery stenosis and spatial inhomogeneity of the arterial wall are the main predictors of the difference between the two methods. Still, the reproducibility of both methods is similar and the correlation is acceptable in this elderly, diseased population. Hence, RF-based measurement of CCAIMT is feasible in clinical practice. 


\section{References}

1. Devine PJ, Carlson DW, Taylor AJ. Clinical value of carotid intima-media thickness testing. Journal of Nuclear Cardiology. 2006;13:710-718

2. Lorenz MW, Markus HS, Bots ML, Rosvall M, Sitzer M. Prediction of clinical cardiovascular events with carotid intima-media thickness: A systematic review and meta-analysis. Circulation. 2007;115:459-467

3. Bots ML, Dijk JM, Oren A, Grobbee DE. Carotid intima-media thickness, arterial stiffness and risk of cardiovascular disease: Current evidence. Journal of hypertension. 2002;20:2317-2325

4. Touboul PJ, Hennerici MG, Meairs S, Adams H, Amarenco P, Bornstein N, et al. Mannheim carotid intima-media thickness consensus (2004-2006). An update on behalf of the advisory board of the $3 \mathrm{rd}$ and 4th watching the risk symposium, 13th and 15th European stroke conferences, Mannheim, Germany, 2004, and Brussels, Belgium, 2006. Cerebrovascular diseases. 2007;23:75-80

5. Hoeks AP, Brands PJ, Willigers JM, Reneman RS. Non-invasive measurement of mechanical properties of arteries in health and disease. Proceedings of the Institution of Mechanical Engineers, part H. 1999;213:195-202

6. Hoeks AP, Willekes C, Boutouyrie P, Brands PJ, Willigers JM, Reneman RS. Automated detection of local artery wall thickness based on M-line signal processing. Ultrasound in medicine \& biology. 1997;23:1017-1023

7. Brands PJ, Hoeks AP, Willigers J, Willekes C, Reneman RS. An integrated system for the non-invasive assessment of vessel wall and hemodynamic properties of large arteries by means of ultrasound. European Journal of Ultrasound. 1999;9:257-266

8. Gamble G, Zorn J, Sanders G, MacMahon S, Sharpe N. Estimation of arterial stiffness, compliance, and distensibility from M-mode ultrasound measurements of the common carotid artery. Stroke. 1994;25:11-16

9. Willekes C, Hoeks AP, Bots ML, Brands PJ, Willigers JM, Reneman RS. Evaluation of off-line automated intima-media thickness detection of the common carotid artery based on M-line signal processing. Ultrasound in medicine \& biology. 1999;25:57-64

10. Van Bortel LM, Vanmolkot FH, van der Heijden-Spek JJ, Bregu M, Staessen JA, Hoeks AP. Does B-mode common carotid artery intima-media thickness differ from M-model? Ultrasound in medicine \& biology. 2001;27:1333-1336

11. Grant EG, Benson CB, Moneta GL, Alexandrov AV, Baker JD, Bluth El, et al. Carotid artery stenosis: Gray-scale and doppler us diagnosis - Society of radiologists in ultrasound consensus conference. Radiology. 2003;229:340-346

12. Meinders JM, Kornet L, Brands PJ, Hoeks AP. Assessment of local pulse wave velocity in arteries using 2D distension waveforms. Ultrasonic Imaging. 2001;23:199-215

13. Hermans MM, Kooman JP, Brandenburg V, Ketteler M, Damoiseaux JG, Tervaert JW, et al. Spatial inhomogeneity of common carotid artery intima-media is increased in dialysis patients. Nephrology, Dialysis and Transplantation. 2007;22:1205-1212

14. Lin LI. A concordance correlation coefficient to evaluate reproducibility. Biometrics. 1989;45:255-268

15. Deyo RA, Diehr P, Patrick DL. Reproducibility and responsiveness of health status measures. Statistics and strategies for evaluation. Control Clinical Trials. 1991;12:142S-158S

16. Bland JM, Altman DG. Statistical methods for assessing agreement between two methods of clinical measurement. Lancet. 1986;1:307-310 
17. Potter K, Reed CJ, Green DJ, Hankey GJ, Arnolda LF. Ultrasound settings significantly alter arterial lumen and wall thickness measurements. Cardiovascular Ultrasound. 2008;6:6

18. Bots ML, Hofman A, Grobbee DE. Increased common carotid intima-media thickness. Adaptive response or a reflection of atherosclerosis? Findings from the Rotterdam study. Stroke. 1997;28:24422447

19. Amarenco P, Labreuche J, Lavallee P, Touboul PJ. Statins in stroke prevention and carotid atherosclerosis: Systematic review and up-to-date meta-analysis. Stroke. 2004;35:2902-2909

20. Kiechl S, Willeit J. The natural course of atherosclerosis. Part I: Incidence and progression. Arteriosclerosis, thrombosis, and vascular biology. 1999;19:1484-1490

21. Kanters SD, Algra A, van Leeuwen MS, Banga JD. Reproducibility of in vivo carotid intima-media thickness measurements: A review. Stroke. 1997;28:665-671

22. Bots ML, Mulder PG, Hofman A, van Es GA, Grobbee DE. Reproducibility of carotid vessel wall thickness measurements. The Rotterdam study. Journal of Clinical Epidemiology. 1994;47:921-930

23. Kanters SD, Elgersma OE, Banga JD, van Leeuwen MS, Algra A. Reproducibility of measurements of intima-media thickness and distensibility in the common carotid artery. European journal of vascular and endovascular surgery. 1998;16:28-35

24. Touboul PJ, Vicaut E, Labreuche J, Belliard JP, Cohen S, Kownator S, et al. Design, baseline characteristics and carotid intima-media thickness reproducibility in the PARC study. Cerebrovascular diseases. 2005;19:57-63 



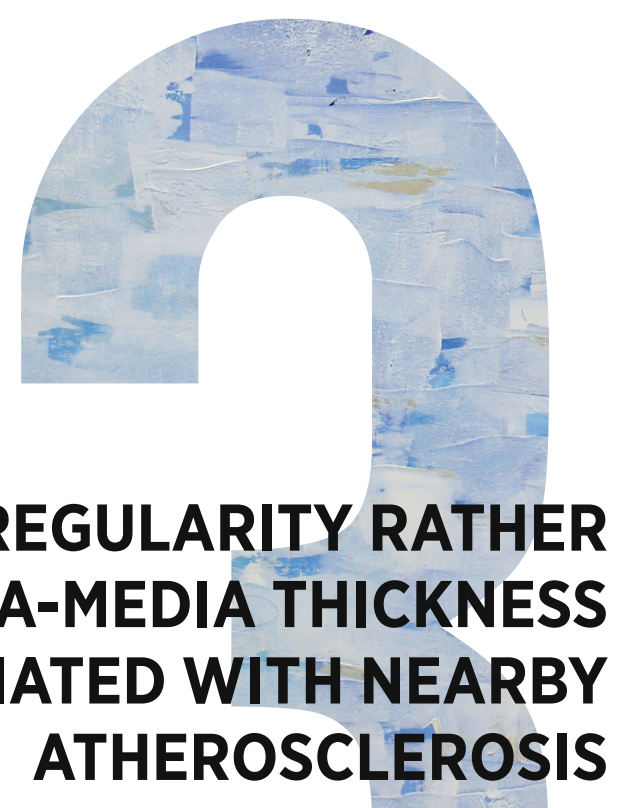

WALL IRREGULARITY RATHER THAN INTIMA-MEDIA THICKNESS IS ASSOCIATED WITH NEARBY ATHEROSCLEROSIS

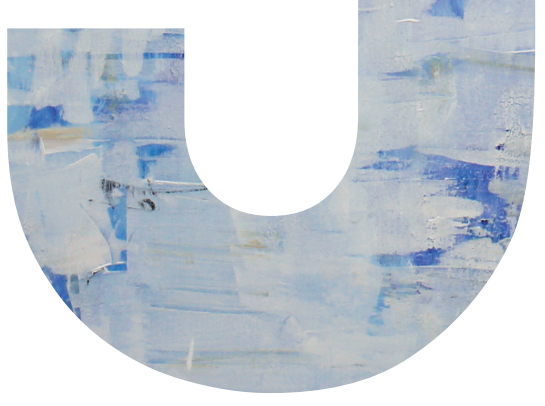

IM Graf, FHBM Schreuder, JM Hameleers, WH Mess, RS Reneman, APG Hoeks

Published in:

Ultrasound in Medicine and Biology 2009; 35(6): 955-961 


\section{Abstract}

In addition to intima-media thickness (IMT), IMT-inhomogeneity may carry information about atherosclerosis progression. In 147 vascular diseased patients (mean 66 years, $48 \%$ male), we determined the carotid bulb stenosis degree based on local Doppler blood flow velocities. Common carotid artery (CCA) morphologic characteristics, i.e. IMT, IMT-inhomogeneity (intraregistration variation) and IMT uni- and bilateral intrasubject variation $(\triangle \mathrm{IMT})$, were measured using multiple M-mode. Associations of morphologic characteristics, stenosis degree and Framingham score were evaluated with Pearson correlation $(r)$ and multiple regression analysis. The IMT distributions for subjects without and with stenosis were not similar. The stenosis degree score correlated significantly to unilateral $(r=0.68)$ and bilateral $\triangle \mathrm{IMT}(r=0.62)$, IMT $(r=0.41)$ and IMT-inhomogeneity $(r=0.45)$. The averaged IMT and IMT-inhomogeneity increased slightly for singular stenosis and abruptly for multiple stenoses. Mean uni- and bilateral $\triangle I M T$ per stenosis degree increased linearly with this degree, reaching a correlation close to $1(r=0.98$ and $r=0.97$ ). Interestingly, the majority of the subjects with a moderate to severe bulb stenosis exhibited a carotid IMT lower than the considered critical threshold of $0.9 \mathrm{~mm}$. In conclusion, although CCA is not prone to plaques, its morphologic characteristics are positively correlated with stenosis degree score and other risk scores. $\triangle \mathrm{IMT}$ can be more reliable derived from inter-registration rather than from intra- registration variation. In the CCA, $\triangle$ IMT substantiates vascular alteration better than IMT. 


\section{Introduction}

Atopic morphologic characteristics of the common carotid artery (CCA) correspond with existence of vascular diseases.' The association between structural abnormalities of large arteries, as assessed by ultrasonographic techniques, and vascular diseases has been a topic of interest in clinical studies. ${ }^{2,3}$

Traditionally, clinicians estimate the 10-year risk of stroke or cardiovascular events, for patients diagnosed with vascular diseases by means of Framingham score (FS) ${ }^{4}$ and Framingham vascular score (FVS) ${ }^{5}$. FS is derived from the following risk factors: gender, age, total cholesterol, high density lipoprotein (HDL)-cholesterol, systolic and diastolic blood pressure, diabetes and smoking status. In addition to the factors included in the calculation of FS, the formula for FVS contains classification based on hypertension treatment, presence of CVD, atrial fibrillation and left ventricular hypertrophy. For further enhancement of risk stratification, the risk scores are combined with parameters derived from ultrasonographic measurements i.e., stenosis degree ${ }^{6}$ or morphologic characteristics of arterial walls such as intima-media thickness (IMT) ${ }^{7}$. Follow-up studies have demonstrated a strong and graded association between IMT and ischemic stroke subtypes ${ }^{8}$, future carotid plaque formation ${ }^{9}$ and incidence of myocardial infarction, stroke and vascular death ${ }^{10}$, suggesting that IMT is a suitable marker to grade and predict atherosclerotic processes.11, 12

Echo techniques used in these studies are restricted to a single assessment of IMT at one point or to an average over an arterial segment of $1-2 \mathrm{~cm} \cdot{ }^{13}$ Assessed in this way, IMT values higher than $0.9 \mathrm{~mm}$ are considered to be indicative of atherosclerotic disease. ${ }^{14}$ However, the approach of a straightforward cut-off for IMT ignores gradual and local changes in vessel wall dimension..$^{15}$ Longitudinal variations in IMT have been shown to be present in healthy subjects ${ }^{16}$ and in end-stage renal disease patients ${ }^{2}$. Irregularities in IMT can be quantified by determining IMT-inhomogeneity, defined as the standard deviation of IMT over a given segment. The latter approach assumes that the considered segment covers several ultrasound resolutions in the lateral direction to obtain independent samples. A similar measure for inhomogeneity can be based on the registration variation for repeated measurements.

A common site for atherosclerotic plaques is the internal carotid artery bulb near the common carotid artery (CCA) bifurcation. Occasionally, the bulb may extend into the CCA up to $2 \mathrm{~cm}$ from the flow divider. The CCA, generally remains free of atherosclerotic plaques, although it may exhibit changes in wall morphology. The aim of the present study was to investigate the interrelationship, if any, between CCA morphologic changes in terms of IMT, IMT-variation and IMT-inhomogeneity and the degree of carotid artery bulb stenosis. The morphologic changes were determined over an arterial segment of $1.6 \mathrm{~cm}$, located approximately $2 \mathrm{~cm}$ proximal to the CCA flow divider, by means of an RF-based ultrasound technique, in a cohort of patients with recent cerebrovascular 
and cardiovascular events. Moreover, these measures were associated to traditional risk scores and risk factors.

\section{Methods}

The study was approved by the joint ethical committee of the Maastricht University and the Academic Hospital Maastricht. All subjects gave written informed consent prior to enrollment.

\section{Population}

The study was performed on Caucasian patients requiring carotid artery ultrasonography because of either cerebrovascular disease (i.e., transient or permanent cerebral ischemia resulting in neurological deficit) or vascular screening (preoperative to cardiac surgery). Excluding patients with carotid endarterectomy, the study population consisted of 153 subjects. By interview, tobacco use, symptoms description and family history of cardiovascular diseases were included in the individual clinical profile. Systolic (SBP) and diastolic blood pressure (DBP) were measured using a semi-automatic device (Dinamap, Tampa, FL, USA). Hypertension (SBP $\geq 140 \mathrm{~mm} \mathrm{Hg}$ and/or DBP $\geq 90 \mathrm{~mm} \mathrm{Hg}$ and/or current use of antihypertensive medication), diabetes mellitus (fasting plasma glucose levels $\geq 7.0 \mathrm{mmol} / \mathrm{L}$ or current use of blood glucose lowering drugs), hypercholesterolemia (fasting blood total cholesterol level $\geq 6.5 \mathrm{mmol} / \mathrm{L}$ and/or low density lipoprotein [LDL]cholesterol level $\geq 4.5 \mathrm{mmol} / \mathrm{L}$ and/or present use of lipid lowering drugs), existing comorbidity and related vascular treatment were systematically recorded. The data set was used for deriving Framingham Score and Framingham Vascular Score., 5

\section{Echo data acquisition}

Noninvasive data acquisition was performed in a room dedicated to ultrasonography with the patients in supine position. The ultrasound examination of the carotid artery involved two steps: (1) duplex ultrasonography of the extracranial carotid arteries, and (2) recording of the constituting radio frequency (RF) data. A single well-trained ultrasonography technician conducted both steps of the data acquisition for the same patient.

\section{Duplex scanning}

The carotid artery was visualized using a color-coded duplex ultrasonographic device (SONOS 5500; Philips Medial Systems, Eindhoven, The Netherlands), equipped with a 7.5 $\mathrm{MHz}$ linear array transducer. The power output was standardized for all measurements. The protocol included a full examination of the extracranial arteries at various locations, imaged in duplex and in color Doppler mode, to establish plaque presence and location. The degree of stenosis was determined for the dominant plaque based on local blood flow velocities, according to the criteria proposed by the Society of Radiologists in 
Ultrasound. ${ }^{14,17}$ The estimated stenosis degree score (none, < 50\%, 50\%-69\%, 70\%-99\% and $100 \%$ stenosis) was converted to correspondingly increasing numbers from 0 to 4 . Any unilateral supplementary plaque observed in the carotids, disjoint from previously counted plaques, increased the stenosis degree score with one unit.

\section{Radio-frequency data acquisition}

Ultrasound RF data were acquired with a Picus-ArtLab ultrasound machine (Esaote Europe, Maastricht, The Netherlands) using a $40 \mathrm{~mm} 7.5 \mathrm{MHz}$ linear array transducer, operating in multiple $\mathrm{M}$-mode with high temporal resolution. The images were composed of $14 \mathrm{M}$-lines recorded at a $33 \mathrm{MHz}$ sample frequency, spaced at $1.2 \mathrm{~mm}$, and a frame rate of $733 \mathrm{~Hz}$ covering three heart beats. The upper end of the transducer was at least $2 \mathrm{~cm}$ proximal to the CCA flow divider. Two repeated and independent measurements were performed on both the left and the right CCA, inter-merged, without the use of a location marker. During ultrasound RF data acquisition, a three-lead ECG signal was simultaneously recorded. The duration of the Picus-ArtLab scanning procedure ranged from 5 to $10 \mathrm{~min}$. The deforming effect of image processing on the detection of the IMTdistribution was avoided by considering the received radio-frequency (RF) signal and using its envelope to detect tissue transitions..$^{18,19}$

\section{Data processing}

The ultrasound RF data were individually recalled and subjected to Hilbert transformation to compute the instantaneous echo amplitude on a linear scale as function of depth and echo line position. Images coincident with the R top of the simultaneously recorded ECG were selected for further analysis. The media-adventitia and lumen-intima transitions were identified manually, by placing punctual markers in the B-mode image with secondary visual support of the corresponding RF lines. The procedure resulted in the estimation of end-diastolic IMT of the posterior wall as function of echo line position and cardiac beat. Subsequently, the IMT values were averaged over all 14 echo lines (segment length $1.6 \mathrm{~cm}$ ) and all cardiac beats. For each beat, the standard deviation of IMT over the echo lines was calculated and referred to as IMT-inhomogeneity. Its average was based on the square root of the average variance over all heart beats.

The variation in IMT measurements was analyzed using intrasubject variation for unilateral and bilateral measurements. Unilateral intrasubject variation of IMT $\left(\triangle I M T_{\text {uni }}\right)$ is defined as the standard deviation (SD) of the difference between the two repeated measurements on either the left or the right CCA, independent of the site of stenosis.

$$
\Delta I M T_{\text {uni }}=S D\left(I M T_{1}-I M T_{2}\right)
$$

Similarly, the IMT bilateral intrasubject variation $\left(\triangle I M T_{b i}\right)$ evaluates the difference between the averaged unilateral IMT values on the right and left side, independent of the site of stenosis.

$$
\Delta I M T_{b i}=S D\left(I M T_{\text {left }}-I M T_{\text {right }}\right)
$$




\section{Statistical analysis}

The influence of various parameters on IMT variation was investigated with multiple linear regression analysis. The association of risk factors and scores with IMT, $\triangle \mathrm{IMT}$ and IMT-inhomogeneity was investigated with Pearson bivariate correlation ( $r$ ). Conditional logistic regression for data sets, normalized to their means, was used to study the slope difference between IMT, $\triangle I M T$ and IMT-inhomogeneity. One-way analysis of variance (ANOVA) was used to test the variance of IMT, $\triangle \mathrm{IMT}$ and IMT-inhomogeneity averaged per stenosis degree group. The significance level was set at $p<0.05$. 


\section{Results}

Ultrasound measurement was successful in 147 patients (96\%). Because not all 147 patients had four successful measurements (two for each side), a total of 483 measurements were finally obtained. Measurement failure was related to inferior visualization quality of the CCA due to tortuous walls or localization of the posterior CCA wall beyond the acquisition range.

The population distribution based on reason for ultrasonography indicates that $42 \%$ were referred in relation to stroke, $23 \%$ for transient ischemia, $7.5 \%$ for ocular ischemia, $7 \%$ for precardiac surgery and the rest for other vascular diseases (e.g., vascular alteration of peripheral arterial segments).

The alteration of carotid arterial walls was evaluated and quantified according to the procedure described in the Methods section. For the investigated population, the maximum stenosis degree score was 7. The highest stenosis degree score was obtained in three cases with $100 \%$ degree of stenosis combined with three additional plaques, and seven cases with stenosis of $50 \%-70 \%$ or $70 \%-90 \%$ combined with maximally five other plaques. All the subjects with more than two unilateral multiple plaques presented a carotid bulb stenosis of more than $50 \%$.

Table 3.1. Clinical and morphological characteristics in combination with risk scores calculated for the entire population, reported as mean values $\pm S D$.

Parameter $\quad \mathrm{N}=154$ patients

\begin{tabular}{|c|c|}
\hline Age (years) & $66 \pm 12$ \\
\hline Sex (\% male) & 48 \\
\hline SBP (mm Hg) & $141 \pm 22$ \\
\hline LDL/HDL & $3.8 \pm 1.6$ \\
\hline Diabetes mellitus (\% yes) & 12 \\
\hline Smoking (\% yes) & 29 \\
\hline BMI & 26 \\
\hline FS (\%) & $4.1 \pm 1.4$ \\
\hline FVS (\%) & $9.2 \pm 7.7$ \\
\hline Stenosis degree score & $1.5 \pm 1.5$ \\
\hline IMT (mm) & $0.71 \pm 0.2$ \\
\hline$\Delta \mathrm{IMT}_{\text {uni }}(\mu \mathrm{m})$ & $59 \pm 49$ \\
\hline$\Delta \mathrm{IMT}_{\mathrm{bi}}(\mu \mathrm{m})$ & $70 \pm 78$ \\
\hline IMT-inhomogeneity $(\mu \mathrm{m})$ & $15 \pm 18$ \\
\hline
\end{tabular}

Abbreviations: BMI, body mass index; FS, Framingham score; FVS, Framingham vascular score; HDL, high density lipoprotein; IMT, intima-media thickness; LDL, Iow density lipoprotein; SBP, systolic blood pressure. 
The clinical profile of the entire population together with the morphological characteristics and risk scores are summarized in Table 3.1.

IMT

The beat to beat intraregistration variation of IMT was $0.1 \pm 0.08 \mu \mathrm{m}$. The means of IMT per stenosis degree score are depicted in Figure 3.1a. A total of 101 measurements pertained to the minimum stenosis degree score, with an average IMT of $0.63 \mathrm{~mm}$. Although the increase of IMT between the stenosis degree groups of one and five is small, linear regression analysis shows that the difference is marginally significant $(p=0.04)$. The limited association between IMT and stenosis degree is also illustrated by histogram analysis (Figure 3.2). Breaking up the population into two groups, without (Figure 3.2a) and with (Figure $3.2 \mathrm{~b}$ ) carotid artery stenosis, demonstrates that on the average IMT is only slightly, though significantly $(p<0.001)$, smaller in the population without carotid artery stenosis ( $0.62 \pm 0.15 \mathrm{~mm}$, mean age 57 years) than in the population with a stenosis score $\geq 1(0.76 \pm 0.18 \mathrm{~mm}$, mean age 66 years). It is of interest to note that in the latter cohort most of the patients do have an IMT substantially lower than the critical threshold of $0.9 \mathrm{~mm}$, although many subjects were referred to ultrasonography because of vascular problems associated with carotid artery stenosis.

\section{IMT-inhomogeneity}

Distribution of IMT-inhomogeneity as mean value per stenosis degree score is depicted in Figure 3.1b. IMT-inhomogeneity appears to increase slightly for stenosis scores from 1 to 5 , being followed by a substantial increase for stenosis scores of 6 and 7 . However, linear regression analysis showed that the increase of mean IMT-inhomogeneity from stenosis degree score 1 to 5 is significant $(p=0.01)$.
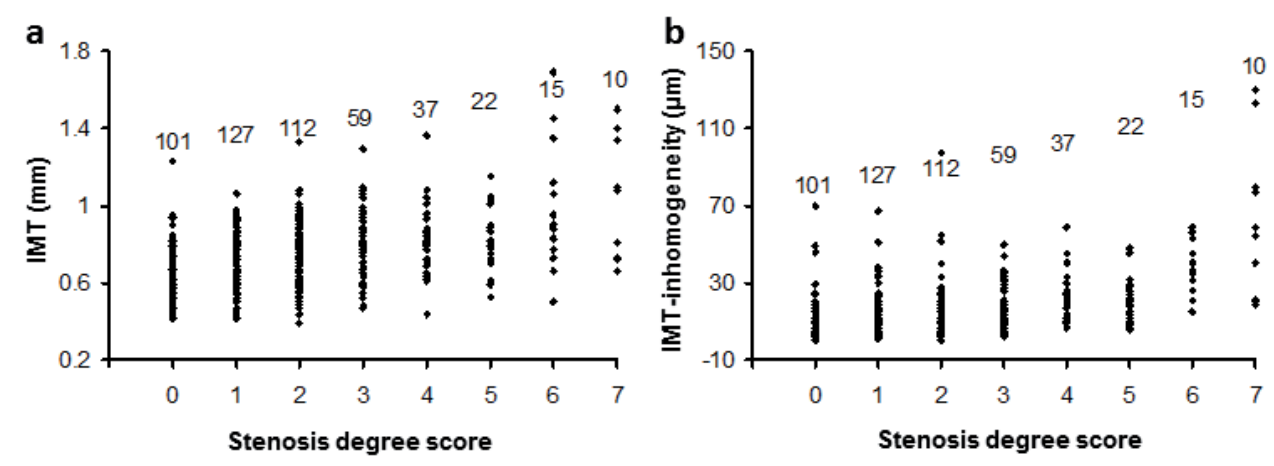

Figure 3.1. Distribution of intima-media thickness (IMT; a) and IMT-inhomogeneity (b) per group, as values (represented by rhombi) and with superimposed mean (marked by horizontal lines), labeled for the number of measurements (total 483). Both IMT and IMT-inhomogeneity increase slightly for stenosis score $\leq 5$, and a pronounced and graded elevation for stenosis scores of 6 and 7 . 
Almost a quarter of the cohort (23\%) had no carotid artery stenosis and an IMTinhomogeneity of $9 \pm 9 \mu \mathrm{m}$. In comparison, the group of patients with carotid artery stenosis had a significantly higher $(p<0.001)$ IMT-inhomogeneity of $16 \pm 11 \mu \mathrm{m}$.

A small percentage of the population showed a discrepancy between the morphological parameters and the stenosis degree score. For example, $3 \%$ of the population exhibited a high stenosis degree score $(>4)$, with either a small IMT $(<0.7 \mathrm{~mm})$ or a low IMTinhomogeneity $(<10 \mu \mathrm{m})$. Similarly, $4 \%$ of the population with limited stenosis degree score $(<2)$ presented a relatively large IMT $(>0.7 \mathrm{~mm}$ ) or large IMT-inhomogeneity $(>15 \mu \mathrm{m})$.

\section{Interregistration variation $(\triangle / M T)$}

Unilateral intrapatient variation, separately on left and right side, revealed no significant difference, allowing data pooling. The $\triangle \mathrm{IMT}_{\text {uni }}$ was $59 \mu \mathrm{m}$ with an intersubject SD of 49 $\mu \mathrm{m}$. Restricting the analysis to the lowest stenosis degree score improved $\Delta \mathrm{IMT}_{\text {uni }}$ to $21 \mu \mathrm{m}$. Multiple regression analysis and Pearson correlation indicated a significant association of $\triangle \mathrm{IMT}_{\text {uni }}$ with unilateral IMT-inhomogeneity $(\beta=0.45, p<0.001$ and $r=0.43, p<0.001$ ).

The difference between the mean IMT of both CCA sides was $3.8 \mu \mathrm{m}$. The mean $\triangle \mathrm{IMT}_{\mathrm{bi}}$ was $70 \mu \mathrm{m}$ with an intersubject SD of $78 \mu \mathrm{m}$. Multiple regression analysis indicated that $\triangle I M T_{b i}$ was strongly related to IMT-inhomogeneity $(\beta=0.68, p<0.001)$. Similarly, Pearson correlation revealed a significant association of $\Delta \mathrm{IMT}_{\mathrm{bi}}$ to unilateral IMT-inhomogeneity $(r$ $=0.51, p<0.001$ ).

The averages of both unilateral $(r=0.98$, Figure 3.3a) and bilateral $(r=0.97$, Figure 3.3b) $\triangle \mathrm{IMT}$ per stenosis degree group were strongly correlated to the stenosis score. Slope analysis of unilateral and bilateral $\triangle I M T$ as functions of stenosis degree illustrates that the associations are significantly different, with the unilateral $\triangle I M T$ exhibiting a more pronounced slope $(p<0.001)$.
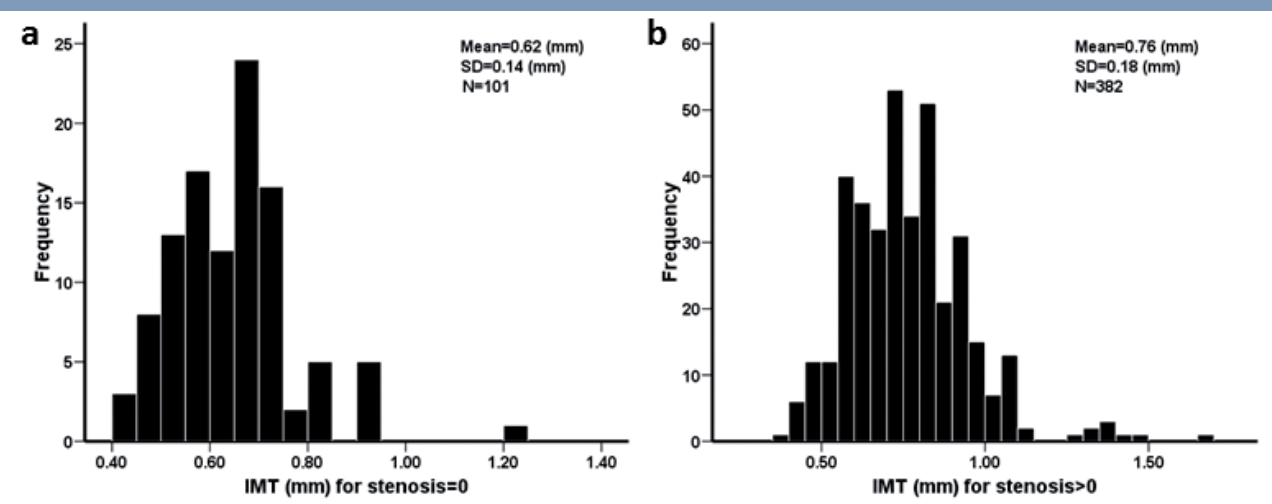

Figure 3.2. Largely overlapping intima-media thickness (IMT) distributions of (a) subpopulation with stenosis degree 0 ( $N=101$ measurements) and (b) subpopulation with stenosis degree >0 ( $N=382$ measurements). The mean IMT of the population without stenosis is slightly smaller than of the population with carotid bulb stenosis. 

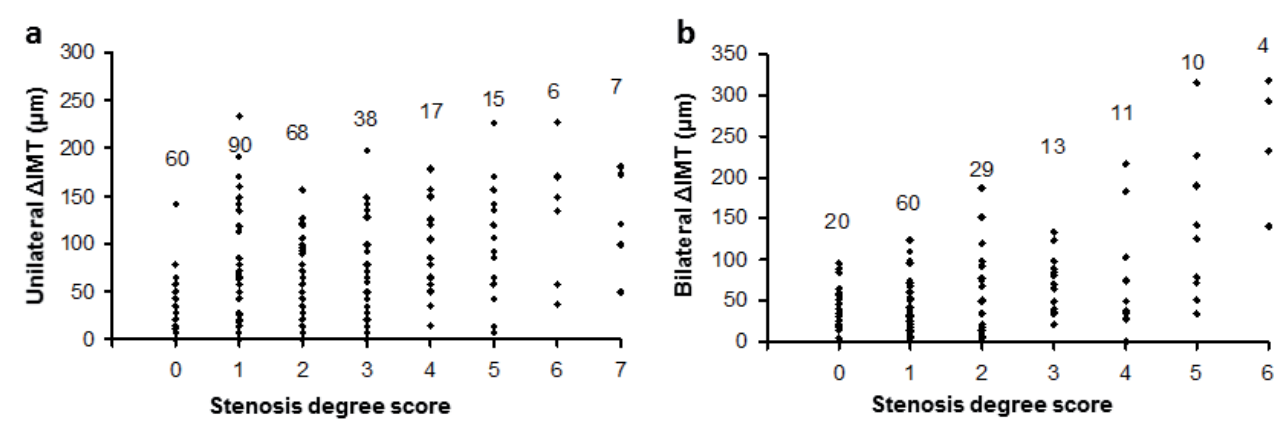

Figure 3.3. Distribution of (a) unilateral and (b) bilateral intrasubject intima-media thickness (IMT)-variation per stenosis degree group, as values (represented by rhombi) and with superimposed mean (marked by horizontal lines), with as bar label the number of measurements and in parentheses the percentage of population per group. Both figures illustrate a strong and graded increase of $\triangle I M T$ with stenosis degree.

The association of IMT, $\triangle \mathrm{IMT}$ and IMT-inhomogeneity with conventional risk factors was explored using Pearson correlation independent of the reason for ultrasonography (Table 3.2). The analyses indicate that IMT, $\triangle I M T$ and IMT-inhomogeneity were positively correlated to stenosis degree and risk scores with the highest correlation for $\triangle I M T$ (unilateral $r=0.68$; bilateral $r=0.62$ ).

Table 3.2. Pearson correlation calculated for unilateral and bilateral IMT, IMT-variation ( $I M T)$ and IMTinhomogeneity ( IMT) in relation to various risk scores for $n=147$ patients. ${ }^{*} p<0.05$; ${ }^{* *} p<0.001$.

\begin{tabular}{lcccccc} 
& \multicolumn{3}{c}{ Unilateral } & \multicolumn{3}{c}{ Bilateral } \\
Parameter & IMT & $\Delta$ IMT & $\sim$ IMT & IMT & $\Delta$ IMT & $\sim$ IMT \\
\hline Stenosis score & $0.41^{* *}$ & $0.68^{* *}$ & $0.45^{* *}$ & $0.24^{* *}$ & $0.62^{* *}$ & $0.27^{* *}$ \\
FS & $0.35^{* *}$ & $0.33^{* *}$ & $0.36^{* *}$ & $0.28^{* *}$ & $0.29^{* *}$ & $0.25^{* *}$ \\
FVS & $0.32^{* *}$ & $0.30^{* *}$ & $0.34^{* *}$ & $0.32^{* *}$ & $0.23^{*}$ & $0.24^{* *}$ \\
\hline
\end{tabular}

Abbreviations: FS, Framingham score; FVS, Framingham vascular score; IMT, intima-media thickness. 


\section{Discussion}

The findings in the present study indicate that in patients suffering from cerebro- or cardiovascular disease, IMT, $\triangle$ IMT (intrasubject variation of IMT) and local IMTinhomogeneity are related to the individual risk of vascular events, as quantified by Framingham score (FS) and Framingham vascular score (FVS), and the degree of carotid artery stenosis, independent of the actual site of stenosis. Of those morphologic parameters, $\triangle I M T$ exhibits the strongest and graded relation to carotid artery stenosis, underlining that $\triangle I M T$ carries different information than IMT and IMT-inhomogeneity.

IMT and local IMT-inhomogeneity show a weak association with the degree of carotid artery stenosis for the majority (70\%) of the population. The larger mean IMT in the population with carotid artery stenosis than in the population without stenosis (Figure 3.2) can be explained by the significantly higher age in the former population. ${ }^{20}$ Only for stenosis degree scores $>4$, corresponding to patients with more than one plaque, the increase of IMT and IMT-inhomogeneity is substantial, leading to a significant association with the stenosis score. Unlike IMT and IMT-inhomogeneity, $\triangle I M T$ shows a consistent graded and significant increase with the degree of carotid artery stenosis (Figure 3.3), reaching a correlation close to 1 for both the unilateral and bilateral mean $\triangle I M T$. The association of the stenosis degree with $\triangle I M T$ is stronger than with IMT-inhomogeneity because IMT-inhomogeneity will reveal only vascular alterations with a size up to the width $(16 \mathrm{~mm})$ of the scan plan. ${ }^{21}$

It has been proposed that an IMT cut-off value of $0.9 \mathrm{~mm}$ could be used as indication of the risk of vascular diseases..$^{14}$ In our cohort of patients with carotid artery stenosis, however, mean IMT is substantially lower than this critical threshold, $24 \%$ of the patients having an IMT $<0.6 \mathrm{~mm}$. In this study, a $7.5 \mathrm{MHz}$ system was used and not a higher emitting frequency because in the majority of our patients the carotid artery bifurcation was located deeper than $2 \mathrm{~cm}$ from the skin, requiring a good penetration depth. The use of a lower emitting frequency does not influence the outcome of our study, because the IMT to be determined far exceeds the resolution of the system. Also the impact for IMT interregistration variation is limited as indicated by the low average variation of $0.03 \mathrm{~mm}$ for stenosis degree 0 .

The risk scores FS and FVS are derived from a set of nonspecific symptoms, while the stenosis degree score and CCA morphologic characteristics are determined directly by means of ultrasonographic investigations. Despite the direct relation between stenosis degree score on the one hand and IMT, $\triangle \mathrm{IMT}$ and IMT-inhomogeneity on the other, CCA morphologic characteristics have been more often related to FS and FVS. It has been suggested that IMT is associated to coronary events as good as FS.22 Our results show that $\triangle I M T$ is correlated less significantly to FS and FVS than to the stenosis degree score. IMT, $\triangle I M T$, IMT-inhomogeneity and stenosis degree score are indicative of the morphology of the arterial wall at a specific moment, while FS and FVS are estimating the risk over a long 
time interval (10 year) based on risk factors. Therefore, CCA morphologic characteristics, especially $\triangle I M T$, together with the degree of carotid artery stenosis should be included in future studies, which monitor regression or progression of atherosclerosis in patients with cardiovascular risk.

The observed association between mean IMT and carotid bulb stenosis is consistent with other studies., 6 It appears that this association is mainly carried by subjects with a high stenosis degree score (Figure 3.1). This finding and our observation that $\triangle I M T$ is significantly related to carotid artery stenosis imply that the formation of atherosclerotic lesions in the carotid bulb is concurrent with wall irregularities in other segments of the carotid artery tree.

The additional consistent value of $\Delta I M T$ emerged during study analysis. As a consequence no strict protocol (e.g., perpendicular scan planes) was followed for the repeated measurements. The measured values of $\triangle \mathrm{IMT}$ are real because no position marker was used for the ultrasound measurement, ensuring independent registrations. In addition, $\Delta I M T_{\text {uni }}$ and $\Delta I M T_{\text {bi }}$ are of the same order of magnitude $(59 \pm 49 \mu \mathrm{m}$ and $70 \pm 78$ $\mu \mathrm{m}$, respectively), corroborating that the observation is due to structural inhomogeneity. Despite the fact that the morphologic parameters may vary with different angles of CCA visualization and with the proximity to the carotid bulb, multiple regression analysis and Pearson correlation underline that the large intrasubject variations cannot be explained by measurement factors, but have to be attributed to regional morphological variations. Hence, both unilateral and bilateral $\triangle I M T$ can be regarded as separate measures to quantify irregularities in wall characteristics, enhancing the clinical relevance of wall irregularity.

The value of IMT in association to cardiovascular risk has been introduced through cross-sectional studies and later verified through longitudinal studies." The present study does not include follow-up data concerning the clinical outcome of the patients. Therefore, in order to substantiate the prognostic relevance of irregularities in CCA morphology, it would be of interest to include IMT in the present ongoing longitudinal studies on the relation between IMT and cardiovascular disease.

In conclusion, the findings of the present study indicate that $\triangle I M T$ (intrasubject variation of IMT) shows a higher correlation with the severity of carotid artery stenosis than IMT or IMT-inhomogeneity. Therefore, it may be considered to add $\triangle$ IMT to the cardiovascular risk scores commonly in use to evaluate patient's risk.

\section{Acknowledgments}

This study was supported by grant ISO42015 of SenterNovem (Ministry of Economic Affairs, The Netherlands). 


\section{References}

1. Touboul PJ, Labreuche J, Vicaut E, Amarenco P, Investigators G. Carotid intima-media thickness, plaques, and Framingham risk score as independent determinants of stroke risk. Stroke. 2005;36:1741-1745

2. Hermans MM, Kooman JP, Brandenburg V, Ketteler M, Damoiseaux JG, Tervaert JW, et al. Spatial inhomogeneity of common carotid artery intima-media is increased in dialysis patients. Nephrology Dialysis Transplantation. 2007;22:1205-1212

3. Saba L, Caddeo G, Sanfilippo R, Montisci R, Mallarini G. CT and ultrasound in the study of ulcerated carotid plaque compared with surgical results: Potentialities and advantages of multidetector row CT angiography. AJNR. American journal of neuroradiology. 2007;28:1061-1066

4. Wilson PW, D'Agostino RB, Levy D, Belanger AM, Silbershatz H, Kannel WB. Prediction of coronary heart disease using risk factor categories. Circulation. 1998;97:1837-1847

5. D'Agostino RB, Wolf PA, Belanger AJ, Kannel WB. Stroke risk profile: Adjustment for antihypertensive medication. The Framingham study. Stroke. 1994;25:40-43

6. Sakaguchi M, Kitagawa K, Nagai Y, Yamagami H, Kondo K, Matsushita K, et al. Equivalence of plaque score and intima-media thickness of carotid ultrasonography for predicting severe coronary artery lesion. Ultrasound in medicine \& biology. 2003;29:367-371

7. Smilde TJ, van den Berkmortel FW, Boers GH, Wollersheim H, de Boo T, van Langen $\mathrm{H}$, et al. Carotid and femoral artery wall thickness and stiffness in patients at risk for cardiovascular disease, with special emphasis on hyperhomocysteinemia. Arteriosclerosis, thrombosis, and vascular biology. 1998;18:1958-1963

8. Touboul PJ, Elbaz A, Koller C, Lucas C, Adrai V, Chedru F, et al. Common carotid artery intima-media thickness and brain infarction: The etude du profil genetique de l'infarctus cerebral (genic) casecontrol study. The genic investigators. Circulation. 2000;102:313-318

9. Zureik M, Touboul PJ, Bonithon-Kopp C, Courbon D, Berr C, Leroux C, et al. Cross-sectional and 4-year longitudinal associations between brachial pulse pressure and common carotid intimamedia thickness in a general population. The EVA study. Stroke. 1999;30:550-555

10. O'Leary DH, Polak JF, Kronmal RA, Manolio TA, Burke GL, Wolfson SK, Jr. Carotid-artery intima and media thickness as a risk factor for myocardial infarction and stroke in older adults. Cardiovascular health study collaborative research group. The New England journal of medicine. 1999;340:14-22

11. Bots ML, Hofman A, Grobbee DE. Increased common carotid intima-media thickness. Adaptive response or a reflection of atherosclerosis? Findings from the Rotterdam study. Stroke. 1997;28:24422447

12. Riley WA, Barnes RW, Applegate WB, Dempsey R, Hartwell T, Davis VG, et al. Reproducibility of noninvasive ultrasonic measurement of carotid atherosclerosis. The asymptomatic carotid artery plaque study. Stroke. 1992;23:1062-1068

13. Reneman RS, Hoeks AP. Noninvasive vascular ultrasound: An asset in vascular medicine. Cardiovascular Research. 2000;45:27-35

14. Grant EG, Benson CB, Moneta GL, Alexandrov AV, Baker JD, Bluth El, et al. Carotid artery stenosis: Gray-scale and doppler US diagnosis--society of radiologists in ultrasound consensus conference. Radiology. 2003;229:340-346

15. Armentano RL, Graf S, Barra JG, Velikovsky G, Baglivo H, Sanchez R, et al. Carotid wall viscosity 
increase is related to intima-media thickening in hypertensive patients. Hypertension. 1998;31:534-539

16. Willekes C, Brands PJ, Willigers JM, Hoeks AP, Reneman RS. Assessment of local differences in intima-media thickness in the human common carotid artery. Journal of Vascular Research. 1999;36:222-228

17. Braun RM, Bertino RE, Milbrandt J, Bray M. Society of Radiologists in Ultrasound Consensus Criteria to a Single Institution). Ultrasound imaging of carotid artery stenosis: Application of the society of radiologists in ultrasound consensus criteria to a single institution clinical practice. Ultrasound $Q$. 2008;24:161-166

18. Van Bortel LM, Balkestein EJ, van der Heijden-Spek JJ, Vanmolkot FH, Staessen JA, Kragten JA, et al. Non-invasive assessment of local arterial pulse pressure: Comparison of applanation tonometry and echo-tracking. Journal of hypertension. 2001;19:1037-1044

19. Rossi AC, Brands PJ, Hoeks AP. Automatic recognition of the common carotid artery in longitudinal ultrasound B-mode scans. Medical Image Analysis. 2008;12:653-665

20. Howard G, Sharrett AR, Heiss G, Evans GW, Chambless LE, Riley WA, et al. Carotid artery intimalmedial thickness distribution in general populations as evaluated by B-mode ultrasound. ARIC investigators. Stroke. 1993;24:1297-1304

21. Meinders JM, Brands PJ, Willigers JM, Kornet L, Hoeks AP. Assessment of the spatial homogeneity of artery dimension parameters with high frame rate 2-D B-mode. Ultrasound in medicine \& biology. 2001;27:785-794

22. Bernard S, Serusclat A, Targe F, Charriere S, Roth O, Beaune J, et al. Incremental predictive value of carotid ultrasonography in the assessment of coronary risk in a cohort of asymptomatic type 2 diabetic subjects. Diabetes care. 2005;28:1158-1162 




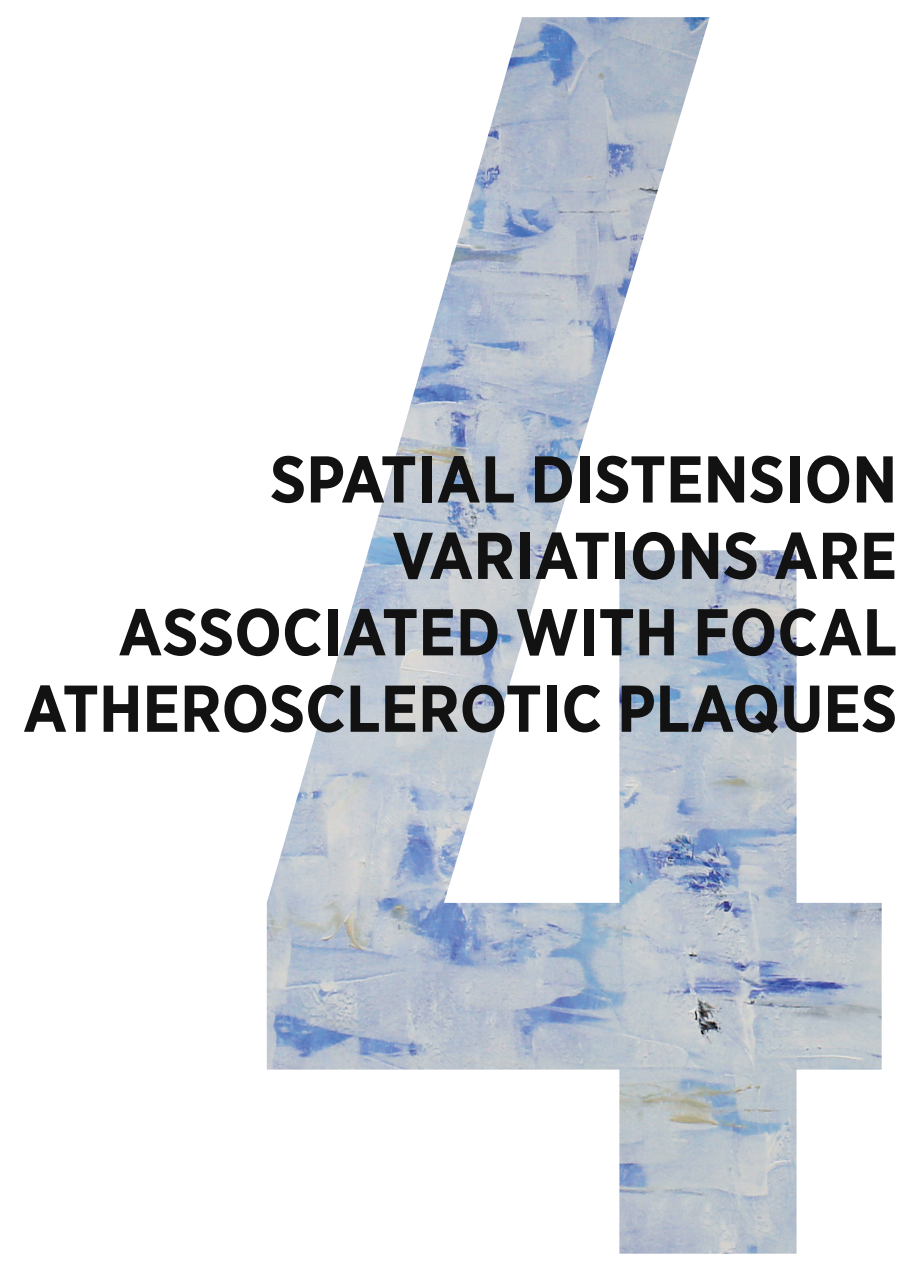

IM Graf, FHBM Schreuder, WH Mess, RS Reneman, APG Hoeks

Published in:

Cerebrovascular Diseases 2010; 29(2): 199-205 


\section{Abstract}

Background: Alterations in arterial absolute distension (AD) and relative distension (RD) in the common carotid artery (CCA), as observed by ultrasound, may relate to atherosclerotic processes in a nearby vascular segment. The main objective of the study was to establish to what extent these mechanical vascular alterations relate to carotid bulb stenosis.

Methods: The AD, RD and their interrecording variation ( $\triangle A D$ and $\triangle R D$ ), representing the spatial inhomogeneity of AD and RD, were evaluated in 308 CCA and associated with the degree of bulb stenosis.

Results: The mechanical CCA parameters were marginally correlated to the degree of ipsilateral ( $r=-0.14$ for $\mathrm{AD}$ and $r=-0.28$ for $\mathrm{RD})$ and contralateral stenosis $(r=-0.21$ for $\mathrm{AD}$ and $r=-0.27$ for RD). However, $\triangle \mathrm{AD}(r=0.54 ; p<0.001)$ and $\triangle \mathrm{RD}(r=0.59 ; p<0.001)$ were significantly correlated to ipsilateral stenosis, independent of age, gender and pulse pressure.

Conclusions: The indices of CCA arterial stiffness are weakly associated with ipsilateral bulb stenosis, but their interrecording variation is a good indicator for atherosclerotic alterations in the carotid bulb. 


\section{Introduction}

Atherosclerosis is associated with hypertrophy of elastic arteries concurrent with an alteration in their mechanical properties.' Surprisingly, the local variation of intima-media thickness of the common carotid artery (CCA) rather than its thickness is more strongly related to the degree of carotid bulb stenosis. ${ }^{2}$ Even though the dynamics of the arterial walls are influenced by age ${ }^{3}$, lifestyle ${ }^{4}$ and drug therapy ${ }^{5}$, they can be used as indicators for atherosclerotic vascular disease. ${ }^{6-8}$

Arterial mechanical properties can be quantified by parameters such as cross-sectional area, local distension, distensibility, cross-sectional compliance coefficient, stiffness index or Young's elastic modulus. These parameters are derived from the arterial diameter and its changes throughout the cardiac cycle. Therefore, the general evaluation of arterial mechanical properties directly depends on the quality of the diameter measurement in space and time. Given the necessity of a reliable assessment of the dynamic arterial diameter, several noninvasive techniques have been proposed for clinical use. ${ }^{9-12}$ Most current approaches are based on an analysis of radiofrequency data combined with autocorrelation ${ }^{13}$ or cross-correlation ${ }^{14}$ methods. The proposed techniques have been validated by reproducibility tests on healthy subjects, for online measurements leading to coefficients of variation of approximately $6 \%$ for the end-diastolic diameter and $8 \%$ for distension." The observed distension variation can be largely attributed to blood pressure variation (respiration and baroreceptor loop), proving the viability of the recording method.

Many studies have investigated the association between atherosclerosis and ischemic events in large populations. ${ }^{15-18}$ However, only a few studies have focused on the mechanical characteristics of the carotid artery, including distension, and atherosclerosis. ${ }^{19}, 20$ A study on cerebrovascular ischemic patients reported a nonsignificant association between plaque existence and distension. ${ }^{19}$ The separation of the population based on the existence of hypertension indicated a significant association $(p<0.001)$ between distension and coexistent plaques in the hypertensive patients, and a weak association in the normotensive group..$^{20}$ But, according to the best of our knowledge, the variation in the arterial mechanical properties of CCA in relation to carotid artery bulb stenosis has not previously been investigated.

This paper presents the mechanical properties of the wall of the CCA and their interrecording variation in relation to the degree of stenosis in the ipsilateral and contralateral carotid bulb in a population of vascular disease patients. The main objective of the study was to establish to what extent these mechanical vascular alterations, as assessed by means of noninvasive ultrasound, relate to carotid bulb stenosis. 


\section{Methods}

The study was approved by the joint ethics committee of Maastricht University and the Academic Hospital Maastricht. All study subjects gave their written informed consent to the examinations after an explanation of the study design.

\section{Study population}

A total of 160 patients were recruited for this study. They were referred to carotid artery ultrasonography because of either cerebrovascular disease (i.e. transient or permanent cerebral ischemia resulting in a neurological deficit) or vascular screening (before cardiac surgery).

Height and weight were recorded, and the body mass index was calculated. Systolic and diastolic blood pressures were measured using a semiautomatic device (Dinamap, Tampa, Fla., USA). Hypertension (systolic blood pressure $\geq 140 \mathrm{~mm} \mathrm{Hg}$ and/or diastolic blood pressure $\geq 90 \mathrm{~mm} \mathrm{Hg}$ and/or current use of antihypertensive medication), diabetes mellitus (fasting plasma glucose level $\geq 7.0 \mathrm{mmol} / \mathrm{l}$ or current use of blood-glucoselowering drugs), hypercholesterolemia (fasting blood total cholesterol level $\geq 6.5 \mathrm{mmol} / \mathrm{I}$ and/or LDL cholesterol level $\geq 4.5 \mathrm{mmol} / \mathrm{I}$ and/or present use of lipid-lowering drugs), existing comorbidity and related vascular treatment were included in the individual clinical profile.

\section{Echo data acquisition}

After 10 min of rest, the ultrasound investigation was performed with the subjects in supine position and the head tilted slightly to the side. A single well-trained ultrasonography technician performed the duplex ultrasound examinations of the extracranial carotid arteries and, subsequently, the radio frequency (RF) data acquisition of the CCA.

\section{Duplex scanning}

The extracranial arteries were visualized using an ultrasonographic device (SONOS 5500; Philips Medial Systems, Eindhoven, The Netherlands) equipped with a 7.5-MHz linear array transducer. The power output was standardized for all measurements. Separately, the carotid bulb and the CCA were imaged in duplex and in color Doppler mode at various locations to establish plaque presence. The degree of stenosis per site was derived from the magnitude of local blood flow velocities, according to the criteria proposed by the 3 consensus meetings for ultrasound methodology. ${ }^{21}$ The stenosis degree intervals (none, < $50 \%, 50-69 \%, 70-99 \%$ and $100 \%$ stenosis) were transformed to numbers ranging from 0 to 4 . For each ipsilateral plaque observed in the carotids (either bulb or common carotid), disjoint from previously counted plaques, the stenosis degree score was increased by 1 unit. 


\section{Radio Frequency data acquisition}

The ultrasound RF signals of the CCA were acquired by a Picus-ArtLab ultrasound machine (Esaote Europe, Maastricht, The Netherlands) using a 40-mm 7.5-MHz linear array transducer that operates in multiple $\mathrm{M}$-mode with a frame rate of $733 \mathrm{~Hz}$, ensuring a high temporal resolution. The images were composed of $14 \mathrm{M}$-lines, spaced at $1.2 \mathrm{~mm}$ and recorded at a 33- $\mathrm{MHz}$ sample frequency, covering 3 heartbeats. The upper end of the transducer was positioned at least $2 \mathrm{~cm}$ proximal to the tip of the CCA flow divider. The lumen was maximized in the longitudinal plane with an optimal image of the near and far walls of the CCA, aiming at obtaining a clear visualization of the typical double lines marking the intima-media layer of the artery.

Two repeated and independent measurements were performed on both the left and the right carotid artery, and then merged (measurement order: left-right-left-right), without the use of a location marker. During ultrasound RF data acquisition, a 3-lead ECG signal was recorded simultaneously.

\section{Data processing}

The mechanical parameters of interest, i.e. absolute distension (AD) and relative distension (RD), were derived by offline processing of the ultrasound RF data, using a specially designed software package. ${ }^{9}$ The ultrasound RF data were individually displayed within a graphic user interface as B-mode images, enabling a manual selection of mediaadventitia wall positions with the corresponding RF lines as a secondary visual support. The selected points were used as initial locations of the wall positions. A cross-correlation tracking technique was applied to estimate the displacement of the walls in time. ${ }^{22}$ The procedure resulted in the assessment of both anterior and posterior wall displacements, from which the diameter waveform for each echo line was derived. Subsequently, the diameter waveforms were averaged over all 14 echo lines (segment length: $1.6 \mathrm{~cm}$ ), providing a single diameter waveform for each recording. The systolic and diastolic diameters averaged over the recording were selected for further analysis.

$A D$ and $R D$ were calculated according to the expert consensus document on arterial stiffness ${ }^{23}$, using the simultaneously recorded ECG as a time reference. AD was defined as the difference between systolic and diastolic diameter, while RD was expressed as the ratio of $A D$ to end-diastolic diameter, both averaged over all (4-6) complete beats within a recording.

The variation in $A D$ and $R D$ measurements was analyzed using intrasubject variation for unilateral measurements. The intrasubject unilateral variation of $A D(\triangle A D)$ is defined as the standard deviation (SD) of the difference between the 2 repeated measurements on either the left or the right CCA, independent of the site of stenosis:

$$
\triangle A D=S D\left(A D_{1}-A D_{2}\right)
$$

Similarly, $\triangle R D$ was calculated as:

$$
\Delta \mathrm{RD}=\mathrm{SD}\left(\mathrm{RD}_{1}-\mathrm{RD}_{2}\right)
$$




\section{Statistical analysis}

The association between the mechanical parameters of the CCA and the degree of ipsilateral or contralateral carotid bulb stenosis was investigated with Pearson's bivariate correlation applied to the entire population (308 arteries). A multiple linear regression model including age, pulse pressure, systolic blood pressure and stenosis degree was used to analyze the association with the mechanical parameters of the CCA.

The statistical analysis was separately effectuated for ipsilateral and contralateral stenosis $(n=308)$ cases. For ipsilateral analysis, the interrecording variation of the right CCA was related to the stenosis score in the right carotid artery bulb, and this was done similarly for the left side. Depending on the purpose of the analysis, the data were further divided, isolating the population without stenosis from the one with stenosis. The unpaired t test including Welch's correction was used to evaluate the significance of the difference between the mechanical arterial parameters of the groups free of and with stenosis, independent of the degree of stenosis. The latter group was divided into 2 subgroups, a moderate stenosis group with a stenosis degree ranging from 1 to 4 , and a severe group with more pronounced carotid artery stenosis. The values of the mechanical parameters were aggregated per stenosis degree group, having the cases with a stenosis degree higher than 4 grouped together. The division was analyzed in terms of general statistics (frequency, mean and SD). The association between stenosis degree and the mean of each of the mechanical characteristics per stenosis degree group was evaluated with the Pearson model. The significance level was set at $p<0.05$. 


\section{Results}

The success rate of the ultrasound measurements was $96 \%$, leaving a total of 154 patients. Measurement failures were due to the inferior visualization quality of the CCA because of tortuous walls or the localization of the posterior CCA wall beyond the acquisition range of the Picus-ArtLab ultrasound machine. Because the mechanical parameters of the CCA and their variation, on the left and right sides, revealed no significant difference (i.e. AD was $0.44 \pm 0.15 \mathrm{~mm}$ on the left and $0.45 \pm 0.11 \mathrm{~mm}$ on the right), the data were pooled, leading to a total of 308 arteries studied separately for ipsilateral and contralateral analysis. No significant differences in the mechanical parameters could be detected between the male and female groups.

The stenosis degree score, calculated according to the procedure described in the Methods section, includes additional plaques located on the bulb $(n=189)$ or CCA ( $n$ $=107$ ). For the investigated population, the maximum stenosis degree score was 7 ( 3 cases) with a $100 \%$ stenosis coexisting with 3 additional plaques, or with a stenosis higher than $50 \%$ and maximally 5 other plaques. All subjects with $>2$ unilateral multiple plaques presented a stenosis of $>50 \%$ in the carotid bulb.

Table 4.1 provides mean values and SD for the clinical and mechanical characteristics. $67 \%$ of patients were hypertensive, $49 \%$ had hypercholesterolemia and $17 \%$ were diagnosed with diabetes. Averaged over the entire population, the AD was $0.45 \pm 0.12$ $\mathrm{mm}$ and the RD $5.2 \pm 1.9 \%$. The mean value of the mechanical parameter variations, $\triangle A D$ and $\triangle \mathrm{RD}$, was $0.07 \pm 0.05 \mathrm{~mm}$ and $1.02 \pm 0.65 \%$, respectively.

Table 4.1. Clinical and mechanical characteristics for the entire study population $(n=154)$.

\begin{tabular}{lc}
\hline Age, years & $66 \pm 12$ \\
\hline Male sex, \% & 48 \\
\hline Systolic blood pressure, $\mathbf{~ m m ~ H g}$ & $141 \pm 22$ \\
\hline Pulse pressure, $\mathbf{~ m m ~ H g}$ & $75 \pm 19$ \\
\hline LDL/HDL & $3.8 \pm 1.6$ \\
\hline Diabetes mellitus, \% & 12 \\
\hline Smoking, \% & 29 \\
\hline BMI & 26 \\
\hline Stenosis degree score & $1.5 \pm 1.5$ \\
\hline Diameters, mm & $7.8 \pm 1.2$ \\
\hline AD, $\mathbf{m m}$ & $0.45 \pm 0.12$ \\
\hline RD, $\%$ & $5.2 \pm 1.9$ \\
\hline$\Delta$ AD, $\mathbf{m m}$ & $0.07 \pm 0.05$ \\
\hline$\Delta$ RD, $\%$ & $1.02 \pm 0.65$
\end{tabular}

Values denote means $\pm S D$, unless otherwise indicated. 


\section{Ipsilateral stenosis}

About a quarter of the subjects studied had no stenosis in the carotid bulb (stenosis degree: 0 ). The number of subjects per stenosis classification decreased inversely with the focal degree of stenosis to 3 cases with the maximal stenosis degree score of 7 . Therefore, for further analysis, the subjects with a stenosis degree higher than 4 were grouped together (Figure 4.1 and Figure 4.2).

Splitting the population up into groups without $(n=82)$ or with $(n=226)$ ipsilateral stenosis revealed a slight but significant $(p<0.001)$ decrease in AD from 0.47 (for the group with no stenosis) to $0.42 \mathrm{~mm}$ (for the group with stenosis degree $>0$ ), and in RD from $5.7 \pm 1.8 \%$ to $5.05 \pm 1.6 \%$, respectively. The interrecording variation of the mechanical parameters of the patient groups varied highly with the degree of stenosis. In the subpopulation without stenosis, $\triangle A D$ and $\triangle R D$ were $0.02 \pm 0.03 \mathrm{~mm}$ and $0.20 \pm 0.07 \%$, respectively. Those values were significantly higher $(p<0.001)$ in the subpopulation with a stenosis: $0.08 \pm 0.06 \mathrm{~mm}$ and $1.1 \pm 0.82 \%$, respectively.

The analysis of the aggregated values of the mechanical parameters per stenosis degree group illustrated a graded decrease with the severity of the vascular alteration (Figure 4.1). The Pearson correlation calculated for the mean values of the mechanical parameters in relation to the stenosis degree indicated a highly significant trend: $r=-0.92$ for $A D$ and $r=-0.95$ for $\mathrm{RD}(p<0.0001)$. The trend analysis of the mechanical parameter variations illustrated a significant increase in both $\triangle \mathrm{AD}(r=0.94 ; \mathrm{p}<0.0001)$ and $\triangle \mathrm{RD}(r$ $=0.97 ; p<0.0001$ ) with stenosis degree (Figure 4.2).

Multiple linear regression analysis $(n=308)$ demonstrated that age and stenosis degree were significantly associated with $A D(\beta=0.65, p<0.001$ and $\beta=0.33, p=$ 0.002 , respectively) and $\operatorname{RD}(\beta=0.62, p<0.001$ and $\beta=0.39, p<0.001$, respectively), while pulse pressure and systolic blood pressure were not significantly dependent. The evaluation of the association between stenosis degree and mechanical CCA parameters with Pearson's correlation indicated that AD was more weakly $(p=0.002)$ associated with stenosis degree than RD (Table 4.2).

The multiple linear regression model, including age, pulse pressure, systolic blood pressure and stenosis degree, indicated that only the last parameter was significantly associated with $\triangle \mathrm{AD}(\beta=0.47 ; p<0.001)$ and $\triangle \mathrm{RD}(\beta=0.51 ; p<0.001)$. 


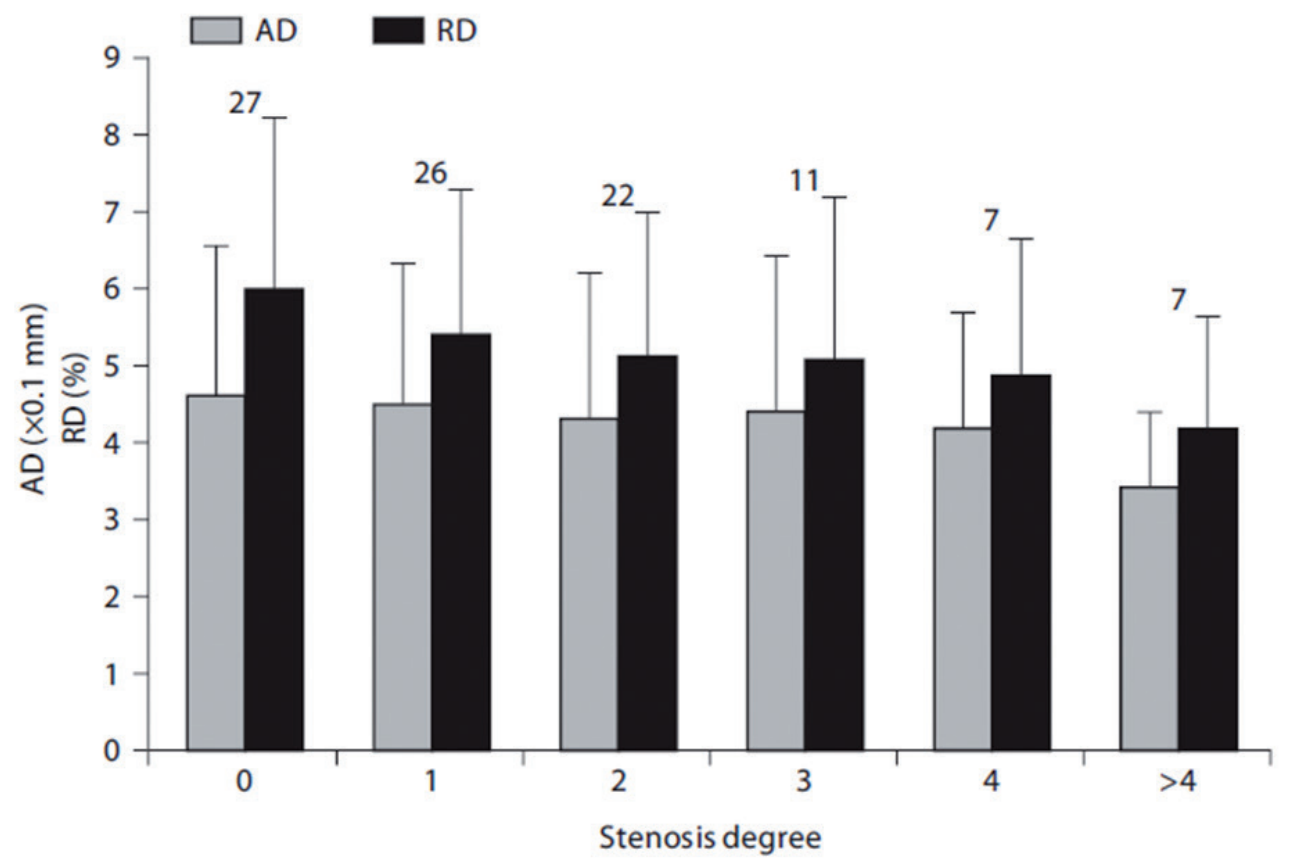

Figure 4.1. Distribution of ipsilateral AD and RD, averaged per stenosis degree score, including SD (whiskers) and the percentage of the population per group (label above whiskers). The 2 parameters exhibit a graded decrease with the stenosis degree.

Pearson's bivariate correlation $(n=308)$ underlined that $\triangle A D$ and $\triangle R D$ were significantly correlated with age ( $\triangle \mathrm{AD}: r=0.28, p<0.001 ; \Delta \mathrm{RD}: r=0.27, p<0.001$ ), but they were more strongly $(p=0.004)$ associated with the ipsilateral stenosis degree (Table 4.2).

The recalculation of the Pearson correlation between stenosis degree and the 4 mechanical parameters adjusted for age leads to an increase in the strength of association only for $\mathrm{AD}$ (from $r=-0.14$ to $r=-0.27 ; p=0.003$ ). The correlation with ipsilateral stenosis decreased for adjusted RD to $r=-0.21$, for adjusted $\triangle \mathrm{AD}$ to $r=0.49$, and for adjusted $\triangle \mathrm{RD}$ to $r=0.38$. Despite the reduced association between stenosis and the 3 age-adjusted mechanical parameters $(\triangle A D, R D$ and $\triangle R D)$, the corresponding $p$ values remained < 0.001 , indicating significant correlation levels. 


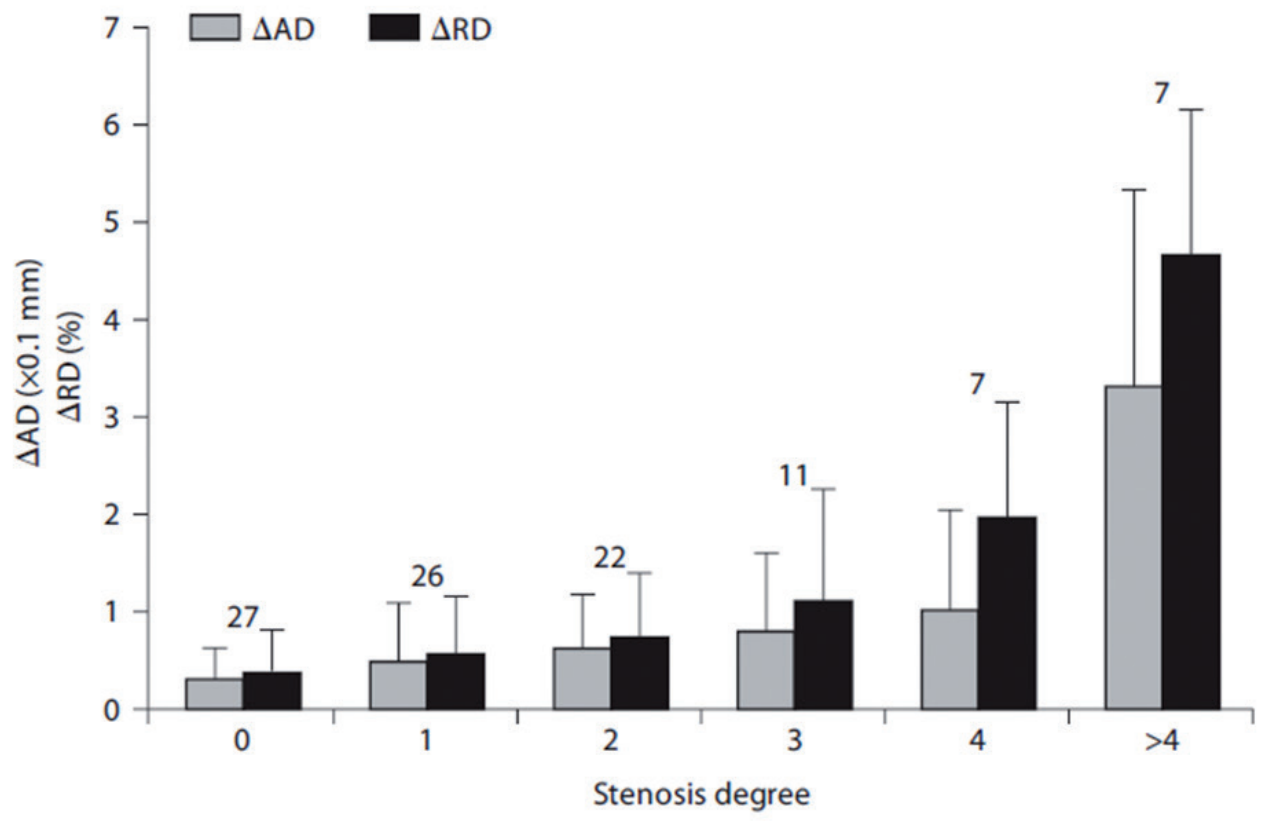

Figure 4.2. Distribution of ipsilateral $\triangle A D$ and $\triangle R D$, averaged per stenosis degree score, including SD (whiskers) and the percentage of the population per group (label above whiskers). In all cases, a significant increase in the ipsilateral variation of the mechanical parameter with the stenosis degree can be observed.

\section{Contralateral stenosis}

The mechanical characteristics of the CCA of the patients with or without contralateral stenosis were compared. The mean AD was higher $(p=0.003)$ in the population without contralateral stenosis $(n=82 ; 0.46 \pm 0.34 \mathrm{~mm})$ than in the population with contralateral stenosis $(n=226 ; 0.44 \pm 0.39 \mathrm{~mm})$. Similarly, the mean RD was higher $(p=0.002)$ in the population without contralateral stenosis $(5.6 \pm 3.7 \%)$ than in the population with contralateral stenosis $(5.2 \pm 4.9 \%)$. In the population without contralateral carotid stenosis, both $\triangle \mathrm{AD}$ and $\triangle \mathrm{RD}(0.03 \pm 0.06 \mathrm{~mm}$ and $0.41 \pm 0.82 \%$, respectively $)$ were significantly lower $(p<0.001)$ than in the population with contralateral stenosis $(0.08 \pm 0.05 \mathrm{~mm}$ and $1.12 \pm 0.93 \%$, respectively).

The analysis of the association between stenosis and the mechanical parameters of the CCA indicated that $A D$ and RD were equivalently correlated with ipsilateral stenosis and with contralaterally a higher (at least 2 points; $n=40$ ) stenosis degree (Table 4.2). In contrast, $\triangle \mathrm{AD}$ and $\triangle \mathrm{RD}$ were marginally but significantly associated with contralaterally a more severe stenosis (AD: $r=0.13, p=0.019$; RD: $r=0.16, p=0.004$ ).

The trend analysis of $A D$ and $R D$ in relation to contralateral stenosis indicated 
significant associations ( $r=-0.85, p=0.007$ and $r=-0.77, p=0.25$, respectively). The correlation of the contralateral stenosis degree with mean $\triangle \mathrm{AD}$ and $\triangle \mathrm{RD}$ was below the significance level ( $r=0.63, p=0.09$ and $r=0.66, p=0.07$, respectively).

Table 4.2. Pearson's correlation for $A D$ and $R D$ and their interregistration variation in relation to stenosis degree score. Left for entire population $(n=308)$, right for subpopulation $(n=40)$ with contralaterally a higher stenosis degree (at least 2 points). ${ }^{*} p<0.05 ;{ }^{* *} p<0.001$

\begin{tabular}{lcccc} 
& \multicolumn{2}{c}{ Ipsilateral } & \multicolumn{2}{c}{ Contralateral } \\
Parameter & parameter & $\Delta$ parameter & parameter & $\Delta$ parameter \\
\hline AD & $-0.14^{* *}$ & 0.07 & $-0.21^{* *}$ & $0.13^{*}$ \\
RD & $-0.28^{* *}$ & 0.09 & $-0.27^{* *}$ & $0.16^{* *}$ \\
\hline
\end{tabular}

Values denote means $\pm S D$, unless otherwise indicated. 


\section{Discussion}

The major finding of the present noninvasive study in a population of vascular disease patients is that the interrecording variation of the $A D$ and RD of the CCA increases with the ipsilateral stenosis degree in the carotid artery bulb, independent of age, gender and brachial artery pulse pressure. This observation indicates that a focal atherosclerotic lesion in the carotid artery bulb is associated with a modification in the dynamic characteristics of a nearby vascular segment. As opposed to conventional parameters describing elastic characteristics, $A D$ and $R D$ are strictly derived from values extracted from the diameter waveform, without including pulse pressure.

It is important to know whether the observed interrecording variation is temporal or spatial (or dual) in nature. If indeed $A D$ and $R D$ do change over time, then their variation will have a systemic origin (e.g. blood pressure variation or neurohumoral stimulation) affecting both sides similarly. According to the protocol, the patients remained in supine position throughout the measurements, and blood pressure and ECG were continuously monitored. Therefore, the temporal variation of physical parameters was limited. Moreover, systemic temporal variations are unlikely because of the similar ipsilateral or contralateral association between stenosis degree and distension inhomogeneity (Table 4.2). Additionally, the absence of a location marker and the merging of the sides of measurement often lead to a visualization of the artery from different insonation angles (i.e. other scan planes). Therefore, independently repeated measurements increase the probability of including inhomogeneities, thus contributing to $\triangle \mathrm{AD}$ and $\triangle \mathrm{RD}$. On the other hand, the mean values of $A D$ and $R D$ hardly vary with the stenosis degree, independent of the recording side (Table 4.2), providing supplemental evidence that the variation is due to spatial inhomogeneities. This finding corroborates an earlier study on the same study population, which showed that the local variation in intima-media thickness rather than its absolute value is strongly related to the degree of carotid bulb stenosis. ${ }^{2}$

The calculation of the stenosis degree score used in our study has not commonly been applied. In standard clinical practice, the assessment of the degree of stenosis is based on peak blood flow velocities. ${ }^{24,25}$ However, multiple plaques involve a vastly distributed vascular alteration. ${ }^{26,27}$ Therefore, by combining peak blood flow velocities with the extent of the atherosclerotic process, in the present study the stenosis degree score covers the characteristics of the vascular alterations in the arterial segment considered.

In our study, carotid distension values were obtained from multiple M-mode measurements. The results showed a high interrecording repeatability of absolute and relative carotid distension for moderate degrees of ipsilateral stenosis, i.e. lower than 3. The current interrecording coefficients of variation for the group free of plaques were similar to those presented in studies focusing on the accuracy of CCA diameter and distension measurements in volunteers ${ }^{11}$ and in patients ${ }^{28}$.

The longitudinal study of Leone et al. presented evidence of an association between 
coronary events and CCA distension and demonstrated that distension is a parameter of interest for cardiovascular disease patients.' Our study investigated the association between distension and existent stenosis in a vascular patient population. The latter association had previously been investigated by Paini et al. in a population with cerebrovascular ischemia, but no significant difference was found between the distension in patients with plaque as compared to the ones without plaque...$^{19}$ The association between distension and coexistent plaques was also analyzed by Beaussier et al., with the distinction that the correlation had been separately calculated for hypertensive and normotensive populations. ${ }^{20}$ Their findings indicate a significant decrease in the mean of $A D$ for the hypertensive group with plaques. The results of the present study in a population mainly formed of hypertensive patients corroborate the data published by Beaussier et al. ${ }^{20}$ The relation between the mechanical characteristics of the CCA and the ipsilateral stenosis degree, as emerges from the group partition, is strongly enhanced if the individual interrecording variation is considered as well. Therefore, it would be of interest to include interrecording distension variation in studies on mechanical arterial properties to reveal vascular alterations.

In the population studied, $A D$ and RD are equivalently associated with ipsilateral and contralateral stenoses. While $A D$ and $R D$ decrease with higher degrees of stenosis, the interrecording variation of distension $(\triangle A D$ and $\triangle R D$ ) is directly proportional to ipsilateral stenosis. In general, when additional plaques are located in the CCA segment considered, a direct effect can be observed on the variability of the measurement. Additional plaques found in the carotid artery bulb also contribute to the stenosis degree score. Thus, the observed relationship between stenosis score and interrecording variation is dependent on plaque location. The statistical analysis indicates that $\triangle A D$ and $\triangle R D$ are less significantly associated with contralateral stenosis. The interrecording variation underlines the necessity of bilateral measurements for a complete evaluation of vascular alterations in the CCA.

Although the present study was not aimed at correlating variations in the mechanical characteristics of the CCA with ischemic events, we believe that our findings are clinically important and should be considered in carotid artery screening. Moreover, they stress the need for detailed evaluation based on repeated and independent observations.

\section{Acknowledgments}

This study was supported by grant ISO42015 of SenterNovem (Ministry of Economic Affairs, The Netherlands). 


\section{References}

1. Leone N, Ducimetiere P, Gariepy J, Courbon D, Tzourio C, Dartigues JF, et al. Distension of the carotid artery and risk of coronary events: The three-city study. Arteriosclerosis, thrombosis, and vascular biology. 2008;28:1392-1397

2. Graf IM, Schreuder FH, Hameleers JM, Mess WH, Reneman RS, Hoeks AP. Wall irregularity rather than intima-media thickness is associated with nearby atherosclerosis. Ultrasound in medicine \& biology. 2009;35:955-961

3. Reneman RS, van Merode T, Hick P, Muytjens AM, Hoeks AP. Age-related changes in carotid artery wall properties in men. Ultrasound in medicine \& biology. 1986;12:465-471

4. Iemitsu M, Maeda S, Otsuki T, Sugawara J, Kuno S, Ajisaka R, et al. Arterial stiffness, physical activity, and atrial natriuretic peptide gene polymorphism in older subjects. Hypertension Research. 2008;31:767-774

5. Lee HW, Karam J, Hussain B, Winer N. Vascular compliance in hypertension: Therapeutic implications. Current Diabetes Reports. 2008;8:208-213

6. Benetos A, Thomas F, Bean K, Gautier S, Smulyan H, Guize L. Prognostic value of systolic and diastolic blood pressure in treated hypertensive men. Archives Internal Medicine. 2002;162:577-581

7. London GM, Cohn JN. Prognostic application of arterial stiffness: Task forces. American Journal of Hypertension. 2002;15:754-758

8. Mattace-Raso FU, van der Cammen TJ, Hofman A, van Popele NM, Bos ML, Schalekamp MA, et al. Arterial stiffness and risk of coronary heart disease and stroke: The Rotterdam study. Circulation. 2006;113:657-663

9. Meinders JM, Hoeks AP. Simultaneous assessment of diameter and pressure waveforms in the carotid artery. Ultrasound in medicine \& biology. 2004;30:147-154

10. Rossi AC, Brands PJ, Hoeks AP. Automatic recognition of the common carotid artery in longitudinal ultrasound B-mode scans. Medical Image Analysis. 2008;12:653-665

11. Morganti T, Ricci S, Vittone F, Palombo C, Tortoli P. Clinical validation of common carotid artery wall distension assessment based on multigate doppler processing. Ultrasound in medicine \& biology. 2005;31:937-945

12. Reneman RS, Meinders JM, Hoeks AP. Non-invasive ultrasound in arterial wall dynamics in humans: What have we learned and what remains to be solved. European heart journal. 2005;26:960-966

13. Rabben SI, Baerum S, Sorhus V, Torp H. Ultrasound-based vessel wall tracking: An auto-correlation technique with rf center frequency estimation. Ultrasound in medicine \& biology. 2002;28:507-517

14. Brands PJ, Hoeks AP, Ledoux LA, Reneman RS. A radio frequency domain complex cross-correlation model to estimate blood flow velocity and tissue motion by means of ultrasound. Ultrasound in medicine \& biology. 1997;23:911-920

15. Kitagawa K, Hougaku H, Yamagami H, Hashimoto H, Itoh T, Shimizu Y, et al. Carotid intima-media thickness and risk of cardiovascular events in high-risk patients. Results of the Osaka follow-up study for carotid atherosclerosis 2 (OSACA2 study). Cerebrovascular diseases. 2007;24:35-42

16. Cardona P, Rubio F, Martinez-Yelamos S, Krupinski J. Endarterectomy, best medical treatment or both for stroke prevention in patients with asymptomatic carotid artery stenosis. Cerebrovascular diseases. 2007;24 Suppl 1:126-133

17. Rothwell PM. Effective stroke prevention in patients with symptomatic carotid stenosis. 
Cerebrovascular diseases. 2004;17 Suppl 1:89-104

18. Li C, Engstrom G, Berglund G, Janzon L, Hedblad B. Incidence of ischemic stroke in relation to asymptomatic carotid artery atherosclerosis in subjects with normal blood pressure. A prospective cohort study. Cerebrovascular diseases. 2008;26:297-303

19. Paini A, Boutouyrie P, Calvet D, Tropeano Al, Laloux B, Laurent S. Carotid and aortic stiffness: Determinants of discrepancies. Hypertension. 2006;47:371-376

20. Beaussier H, Masson I, Collin C, Bozec E, Laloux B, Calvet D, et al. Carotid plaque, arterial stiffness gradient, and remodeling in hypertension. Hypertension. 2008;52:729-736

21. Hennerici M, Baezner H, Daffertshofer M. Ultrasound and arterial wall disease. Cerebrovascular diseases. 2004;17 Suppl 1:19-33

22. Brands PJ, Hoeks AP, Willigers J, Willekes C, Reneman RS. An integrated system for the non-invasive assessment of vessel wall and hemodynamic properties of large arteries by means of ultrasound. European Journal of Ultrasound. 1999;9:257-266

23. Laurent S, Cockcroft J, Van Bortel L, Boutouyrie P, Giannattasio C, Hayoz D, et al. Expert consensus document on arterial stiffness: Methodological issues and clinical applications. European heart journal. 2006;27:2588-2605

24. Schreuder FH, Mess WH, Hoeks AP. Ageing affects the accuracy of duplex ultrasonography in grading carotid artery stenosis. Cerebrovascular diseases. 2009;27:75-83

25. Braun RM, Bertino RE, Milbrandt J, Bray M, Society of Radiologists in Ultrasound Consensus Criteria to a Single Institution Clinical P. Ultrasound imaging of carotid artery stenosis: Application of the society of radiologists in ultrasound consensus criteria to a single institution clinical practice. Ultrasound Q. 2008;24:161-166

26. Nagai Y, Kitagawa K, Yamagami H, Kondo K, Hougaku H, Hori M, et al. Carotid artery intima-media thickness and plaque score for the risk assessment of stroke subtypes. Ultrasound in medicine \& biology. 2002;28:1239-1243

27. Sakaguchi M, Kitagawa K, Nagai Y, Yamagami H, Kondo K, Matsushita K, et al. Equivalence of plaque score and intima-media thickness of carotid ultrasonography for predicting severe coronary artery lesion. Ultrasound in medicine \& biology. 2003;29:367-371

28. Dijk JM, Algra A, van der Graaf Y, Grobbee DE, Bots ML, group Ss. Carotid stiffness and the risk of new vascular events in patients with manifest cardiovascular disease. The SMART study. European heart journal. 2005;26:1213-1220 


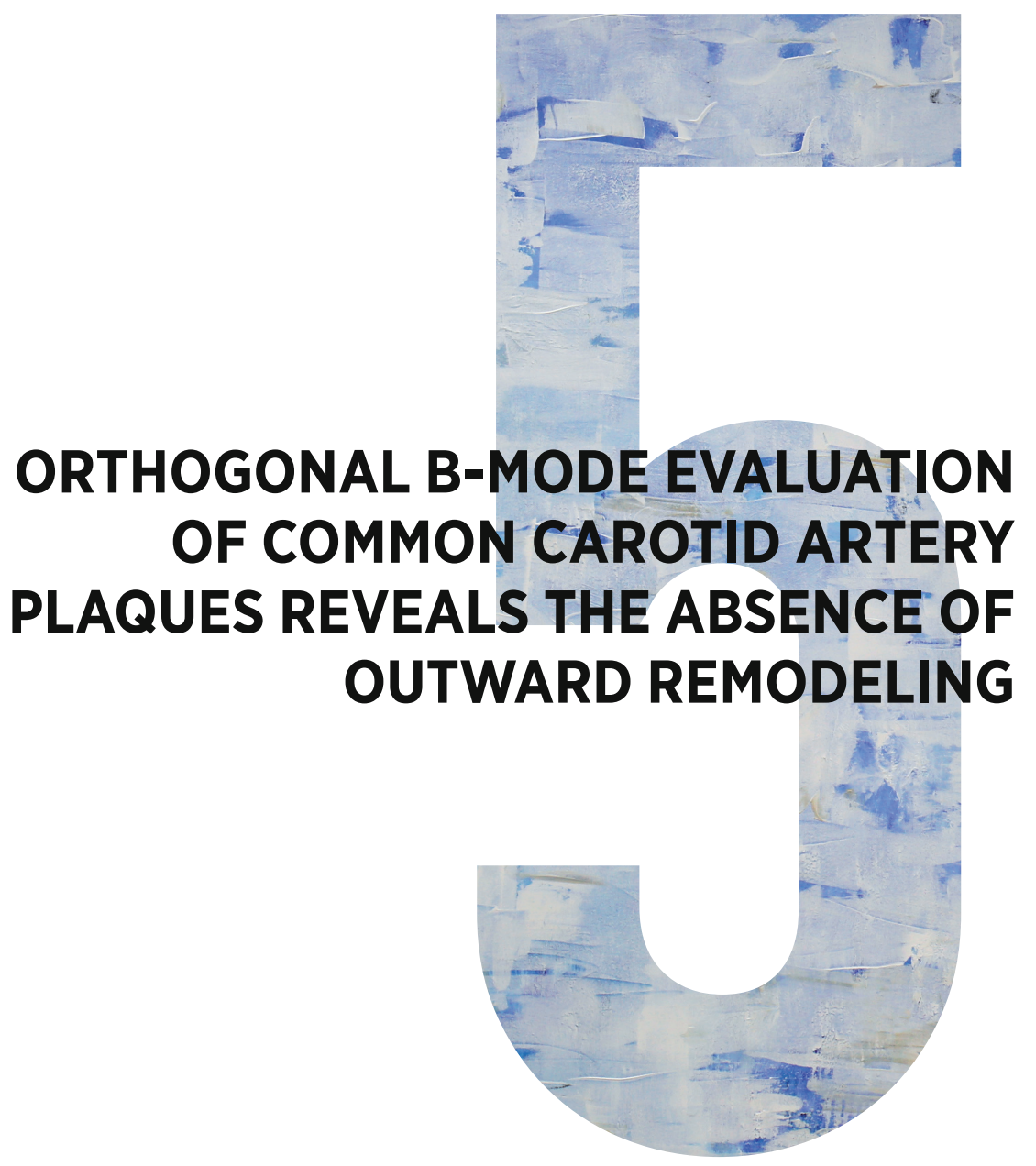

J Steinbuch, FHBM Schreuder, KD Reesink, APG Hoeks, WH Mess

Published in:

Ultrasound in Medicine and Biology 2018; 44(5): 986-994 


\section{Abstract}

To properly assess morphologic and dynamic parameters of arteries and plaques, we propose the concept of orthogonal distance measurements, that is, measurements made perpendicular to the local lumen axis rather than along the ultrasound beam (vertical direction for a linear array). The aim of this study was to compare orthogonal and vertical artery and lumen diameters at the site of a plaque in the common carotid artery (CCA). Moreover, we investigated the interrelationship of orthogonal diameters and plaque size and the association of artery parameters with plaque echogenicity. In 29 patients, we acquired a longitudinal B-mode ultrasound recording of plaques at the posterior CCA wall. After semi-automatic segmentation of end-diastolic frames, diameters were extracted orthogonally along the lumen axis. To establish inter-observer variability of diameters obtained at the location of maximal plaque thickness, a second observer repeated the analysis (subset $N=21$ ). Orthogonal adventitia-adventitia and lumen diameters could be determined with good precision (coefficient of variation: $1 \%-5 \%$. However, the precision of the change in lumen diameter from diastole to systole (distension) at the site of the plaque was poor (21\%-50\%). The orthogonal lumen diameter was significantly smaller than the vertical lumen diameter $(p<0.001)$. Surprisingly, the plaques did not cause outward remodeling, that is, a local increase in adventitia-adventitia distance at the site of the plaque. The intra- and inter-observer precision of diastolic-systolic plaque compression was poor and of the same order as the standard deviation of plaque compression. The orthogonal relative lumen distension was significantly lower for echogenic plaques, indicating a higher stiffness, than for echolucent plaques $(p<0.01)$. In conclusion, we illustrated the feasibility of extracting orthogonal CCA and plaque dimensions, albeit that the proposed approach is inadequate to quantify plaque compression. 


\section{Introduction}

An understanding of mechanical plaque properties would be beneficial in improving plaque risk assessment. In a stroke population, the adventitia-adventitia distension of the common carotid artery (CCA) and the internal carotid artery (ICA) is significantly lower at the stenotic side than at the contralateral side, indicating a stiffer vessel.' Furthermore, decreased distension at plaque location is associated with advanced plaques (containing a lipid core, hemorrhage, calcifications or thrombus), as characterized by magnetic resonance imaging. ${ }^{2}$

A plaque induces pressure wave reflections. ${ }^{3}$ Moreover, it locally increases blood flow velocity and, thereby, wall shear stress, stimulating the endothelium to release vasodilators to restore local wall shear stress to a normal level. ${ }^{4-6}$ As a consequence, it is expected that the adventitia-adventitia diameter at the plaque is larger, which is referred to as outward remodeling. Indeed, in patients with CCA plaques who have type 2 diabetes, dyslipidemia or hypertension, a decreased adventitia-adventitia distension and larger adventitia-adventitia diameter were found at the site of the CCA plaque.,

In standard transcutaneous ultrasound applications, echoes and scattered signals from tissue transitions are received as a function of depth. Therefore, distance or displacement measurements of echo transitions to quantify (e.g. lumen diameter or wall thickness) are performed along the ultrasound beam (vertical direction for a B-mode linear array image), also ensuring best depth resolution. For tissue structures parallel to the skin surface (e.g. the CCA) distance measurements along the ultrasound beam will hardly deviate from distance measurements perpendicular to the local artery axis, even for a deviation of $10^{\circ}$ from perpendicular insonation. However, for tortuous structures oriented oblique to the skin surface (e.g. the carotid bifurcation and internal carotid artery [ICA]), distance and displacement measurements along the ultrasound beam increasingly lose their relevance depending on the angle between the ultrasound beam and the orientation of the structure under examination. Especially the presence of plaques, where the local relevant tissue orientation may reach angles of $45^{\circ}$ with respect to the beam direction, may lead to large differences between orthogonal distance assessment (i.e. perpendicularly to the local lumen axis) and vertical distance assessment.

For proper assessment of local adventitia-adventitia and lumen diameters and distensions, distances between echo transitions should preferentially be measured perpendicular to the local orientation of the blood vessel or lumen axis (Figure 5.1). In this study, we propose the concept of orthogonal distance measurements and apply it to morphologic CCA (plaque) measurements. The procedure starts with semi-automatic outlining of the anterior and posterior lumen-intima and media-adventitia transitions. Subsequently, based on an iterative search for the shortest cross-sectional diameter, the local radius orientation and associated distances are automatically extracted at an interspacing of $1 \mathrm{~mm}$ along the vessel (Figure 5.1). The procedure is executed for the 
external (adventitia-adventitia) and internal (lumen) boundary positions in both diastolic and systolic images of a B-mode video, providing estimates of diastolic-to-systolic changes.

The dynamic parameters (i.e. lumen and adventitia-adventitia distensions and plaque compression) are likely influenced by plaque composition and the pressure wave. Plaque composition can be qualified by plaque echogenicity, the latter quantified by the (normalized) grey-scale median (GSM)., 10 Echolucent plaques are associated with increased risk of cerebrovascular events.11-14 Patients with recurrent ischemic events have a plaque with a large lipid core and lower echogenicity ${ }^{15}$, whereas plaque echogenicity increases with time from stroke or transient ischemic attack (TIA) onset. ${ }^{16}$ The plaque composition has consequences for the local dynamic behavior of lumen and plaque.

The aim of this study was to introduce the concept of orthogonal measurements and to investigate the association between dynamic parameters and plaque echogenicity for plaques in the common carotid artery. More specifically, we (i) evaluated the intraand interobserver precision of morphologic and dynamic parameters at the site of the plaque (i.e. adventitia-adventitia diameter, lumen diameter and distension and plaque compression); (ii) evaluated the difference between orthogonal and vertical mean lumen diameter along the plaque; (iii) considered plaque thickness and compared parameters at the site of the plaque with those of the adjacent proximal or distal segments; and (iv) associated dynamic plaque parameters with normalized grey-scale values specifically for plaques with a high echogenicity.

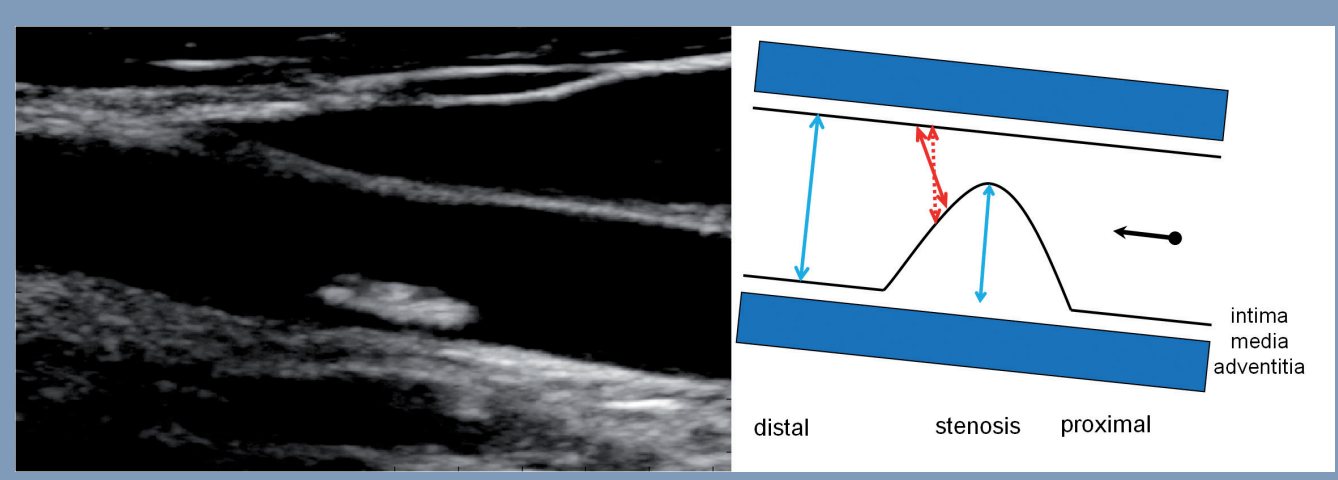

Figure 5.1. For a B-mode image (left), distances are commonly measured along the ultrasound beam, that is, in the vertical direction. However, in an oblique observation (right), distances will be overestimated (dashed red arrow). Rotation of the radial around its center until the shortest distance is reached will reveal the true distance (solid red arrow). This will lead to a proper estimation of the orifice. Note that the degree of stenosis follows from the plaque thickness and unaffected lumen diameter (b/ue arrows). Flow is from right (proximal) to left (distal). 


\section{Methods}

\section{Study subjects}

The Plaque at Risk (PARISK) study (clinicaltrials.gov NCT01208025) is an ongoing multicenter cohort study with 2-y follow-up..$^{17}$ The study was approved by the medical ethics committees of the participating centers, and all patients gave written informed consent. For the current sub-study, we selected the B-mode ultrasound recordings of 29 patients ( $70 \pm 9$ years) with at least one plaque present at the posterior wall within either the left or right CCA. Subject characteristics and relevant clinical data (e.g. age, gender, clinical reason for ultrasound examination) were collected.

Table 5.1. Patient characteristics

\begin{tabular}{lc} 
N & 23 \\
Age (years) & $70 \pm 9$ years \\
\hline Male sex & $15(65 \%)$ \\
\hline Degree of stenosis & $48 \pm 13 \%$ \\
\hline Plaque location & \\
$-\quad$ Posterior wall only & $8(35 \%)$ \\
$-\quad$ Both walls & $15(65 \%)$ \\
\hline Indication & $7(30 \%)$ \\
$-\quad$ Stroke & $7(30 \%)$ \\
$-\quad$ Transient ischemic attack & $1(4 \%)$ \\
$-\quad$ Amaurosis fugax & $8(35 \%)$ \\
\hline
\end{tabular}

Data are presented as the mean \pm standard deviation or number (\%).

\section{Data acquisition}

During the ultrasound examination, the patient lied in supine position with the head slightly tilted to the side opposite that was investigated. A Philips iU22 scanner (Philips Medical Systems, Bothell, WA, USA) with a 9- to 3-MHz linear array probe (width 40 $\mathrm{mm}$ ), operating at a frame rate of approximately 40 frames/s, was used to locate the CCA plaque and to select a plane of observation with maximum plaque cross section. Subsequently, a longitudinal B-mode ultrasound recording of about $6 \mathrm{~s}$ was acquired.

Image analysis: manual edge tracking

To identify end-diastolic and systolic frames, an artery section proximal or distal to the plaque (or with a minimum of plaque interference) is selected. This section is divided into half-overlapping segments with a width of $2 \mathrm{~mm}$, which is on the order of two times the lateral ultrasound resolution (about $1.3 \mathrm{~mm}$ ). The diameter waveform of each segment is 
extracted by automatic edge tracking of the media-adventitia transitions of the anterior and posterior wall using a threshold of $65 \%$ of the maximal grey value of the local adventitia, as previously described. ${ }^{18,19}$ The end-diastolic and systolic frames follow from the local temporal minima and maxima of the spatial average of the diameter waveforms of all segments.

Before manually outlining an artery, image frames are interpolated by a factor of 2 in depth and width, resulting in a pixel size of $33 \times 33 \mu \mathrm{m}$. Subsequently, the positions of the lumen-intima and media-adventitia transitions for both the anterior and posterior walls are manually identified (Figure 5.2) for the first diastolic frame at 1-mm spacing (note: smaller than the lateral resolution). Media-adventitia and lumen-intima transitions are smoothed with a Savitsky-Golay filter of the third order and with a span of seven wall markers (7 mm), providing continuous outlines (Figure 5.2). Next, a first guess is made for the adventitia-adventitia and lumen centerline by averaging corresponding anterior and posterior endpoints along the (vertical) depth axis. Subsequently, the anterior and posterior endpoints are shifted in opposite direction (diameter rotation) until the distance between anterior and posterior outlines reaches a minimum (Figure 5.1), providing the orthogonal orientation and orthogonal diameters. If either endpoint enters a margin of $3 \mathrm{~mm}$ at any side of the image, it is excluded. Finally, the centerlines are recalculated. The final result (Figure 5.2) serves as a template for the next end-diastolic or systolic frame, which improves precision and reduces processing time. The local wall and (hence) plaque thickness is defined as the difference between the media-adventitia and lumenintima transitions along the orthogonal orientation at the anterior or posterior wall, respectively. The relevant plaque segment is automatically extracted by considering the smallest $40 \%$ of orthogonal lumen diameters along the artery, providing an estimate for the mean stenosis diameter. Subsequently the vertical diameter is computed for the same segment. The local orthogonal adventitia-adventitia and lumen distensions are the differences between corresponding orthogonal systolic and end-diastolic diameters. Relative distension is the ratio of distension to end-diastolic diameter. The diameter and distension are determined both at the location of maximal plaque thickness and over the entire image segment by taking the median over all orthogonal diameters or distensions. The plaque compression at the posterior wall is defined as the difference between systolic and end-diastolic vessel wall thickness at the location of maximal plaque thickness. All parameters are averaged (median) over all available heartbeats.

\section{Grey-scale median analysis}

Grey-scale analysis is performed by a trained observer blinded to the results of artery outlining. At each end-diastolic and systolic frame, the plaque contour is manually segmented. For normalization, a reference box is positioned at an unaffected site and covering both the adventitia and lumen (Figure 5.2). The grey value of the lumen within the reference box is set to 0 , and the adventitia value is set to 199. Grey-scale values of the 

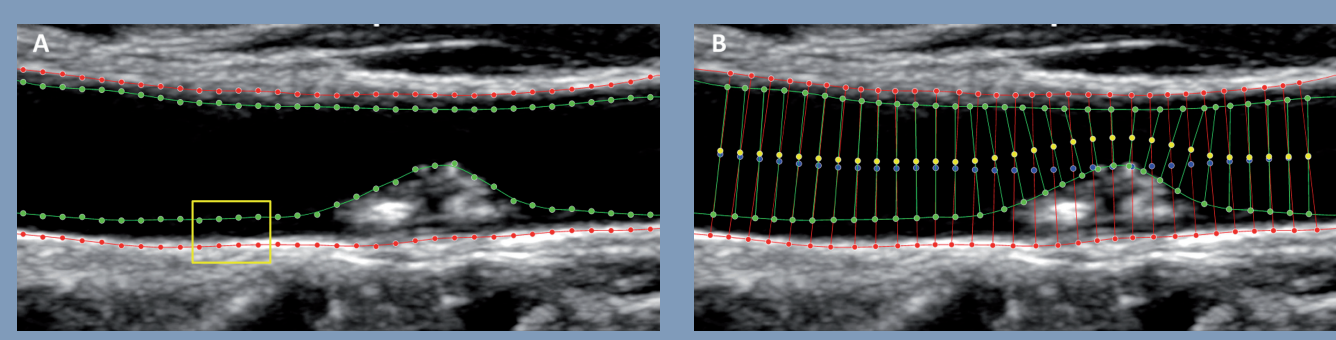

Figure 5.2. Manual outlining (left) at an interspace of $1 \mathrm{~mm}$ of the anterior and posterior lumen-intima (green dots) and media-adventitia (red dots) transitions of a common carotid artery plaque is followed by automatic extraction (right) of the local radius orientation (red and green lines) and associated distances using the lumen (yellow dots) or adventitia-adventitia (b/ue dots) centerlines. The grey-scale references are based on the lumen and adventitia values within the yellow box.

plaque are normalized accordingly. The calcium content of the plaque likely determines most of the local vessel stiffness and is reflected by a higher echogenicity. The latter is quantified arbitrarily by the $75^{\text {th }}$ percentile of the normalized grey value (GS75), and averaged (median) over all heartbeats.

\section{Statistical analysis}

Manual outlining of the CCA was performed twice by an observer without knowledge of the grey-scale results. The analysis was repeated by a second observer for a subset of the patient population. Intra- and inter-observer precision of morphologic and dynamic parameters (i.e., adventitia-adventitia and lumen diameters and distensions) and plaque compression was quantified by the standard deviation of the differences between the observed and assumed ground truth, that is, the observed subject average. A paired t-test was used to compare the mean orthogonal and vertical lumen diameters for the plaque segment and to compare the orthogonal diameter and plaque thickness with those of proximal and distal segments with an average intima-media thickness $<1500$ $\mu \mathrm{m}$. The relation between parameters at maximal plaque thickness and the $75^{\text {th }}$ percentile of the normalized grey-scale value (GS75) was tested with Pearson's correlation and Student's $t$-test. The significance level was set at $p<0.05$. Values are presented as the mean \pm standard variation (SD). 


\section{Results}

Initially the recordings of 6 patients were excluded for insufficient image quality $(\mathrm{N}=$ 2 ), unclear media-adventitia wall because of a shadow $(\mathrm{N}=1)$ or out-of-plane motion of the plaque $(\mathrm{N}=3)$. In total, CCA recordings of 23 patients (age $70 \pm 9$ years) were considered. The degree of stenosis according to the European Carotid Surgery Trial (ECST) criterion was $48 \pm 13 \%$ (Figure 5.1). ${ }^{20}$ Patient characteristics are listed in Table 5.1.

Figure 5.3 provides an illustrative example of the observed external and internal artery diameter distributions and their diastolic to systolic changes for the B-mode recording of Figure 5.2. The change in lumen diameter at the site of the plaque exhibits an inconsistent distribution (from right to left locally lumen extension followed by compression), caused by axial plaque motion.

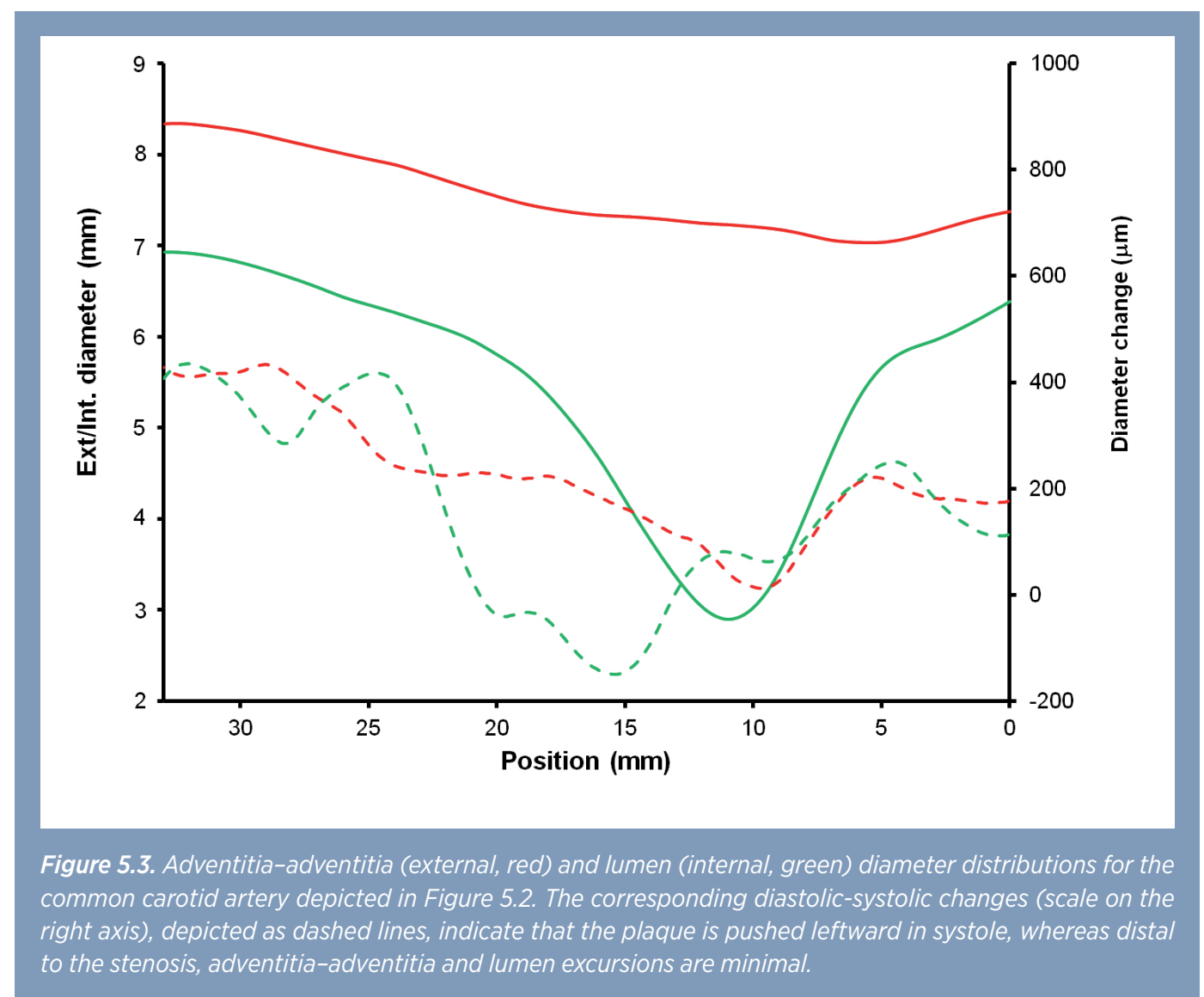

Intra- and inter-observer precision

The mean morphologic parameters and their intra- and inter-observer precision for a subset of 15 patients are summarized in Table 5.2 and Table 5.3. The distribution of values 
observed over the entire artery segment and at the site of maximal plaque thickness does not exhibit any trend with respect to the mean value observed by a single or both observers. The intra- and inter-observer precision of diameters is good (coefficient of variation [CV]: 1\%-5\%), whereas the precision of distension at the plaque center is poor (CV: $21 \%-50 \%$ ). Except for plaque compression, intra-observer precision of all parameters is considerably smaller than inter-observer precision. The intra- and inter-observer CVs of lumen diameter ( $2 \%$ and $5 \%$ ) and distension (28\% and $41 \%$ ), as obtained over the entire artery segment, are larger than those of the adventitia-adventitia diameter ( $1 \%$ and $3 \%$ ) and its distensions (21\% and 39\%). Both intra-observer precision and inter-observer precision of adventitia-adventitia and lumen diameters and distensions are smaller than the standard deviation of our patient population. However, intra-observer precision and inter-observer precision of plaque compression are higher than the mean compression and are of the same order as the standard deviation of plaque compression of our patient population. Therefore, assessment of plaque compression is inadequate and will not be considered for further analysis.

Table 5.2. Intra-observer precision and mean values of diameter and distension estimates $(N=15)$

Mean $(\mu \mathrm{m})$

Intra-obserser

precision $(\mu / m)$

Entire artery segment
Coefficient of variation (\%)

\begin{tabular}{|c|c|c|c|}
\hline & \multicolumn{3}{|c|}{ Entire artery segment } \\
\hline \multicolumn{4}{|l|}{ Adventitia-to-adventitia } \\
\hline End-diastolic diameters & $8822 \pm 724$ & 59 & 1 \\
\hline Distension & $193 \pm 128$ & 40 & 2 \\
\hline \multicolumn{4}{|l|}{ Lumen } \\
\hline End-diastolic diameters & $5351 \pm 1030$ & 107 & 2 \\
\hline Distension & $213 \pm 128$ & 59 & $2 \varepsilon$ \\
\hline
\end{tabular}

At maximal posterior plaque thickness

\begin{tabular}{lccc}
\hline Adventitia-to-adventitia & & & \\
$\quad$ End-diastolic diameters & $8932 \pm 864$ & 78 & 1 \\
$\quad$ Distension & $200 \pm 160$ & 62 & 31 \\
\hline Lumen & & & 3 \\
$\quad$ End-diastolic diameters & $4078 \pm 1049$ & 113 & 31 \\
$\quad$ Distension & $216 \pm 126$ & 67 & 1763 \\
\hline Plaque compression & $8 \pm 113$ & 141 & \\
\hline
\end{tabular}

Orthogonal adventitia-adventitia and lumen diameters can be determined with good intra-observer precision, whereas the precision of distension is poor. However, intra-observer precision of plaque compression is larger than the mean compression and is of the same order as the standard deviation of plaque compression of our patient population. Therefore, plaque compression cannot be precisely determined. Values are presented as the mean \pm standard deviation. Intra-observer precision is defined as the standard deviation of differences between repeated measurements and their average. 
Table 5.3. Diameter and distension determined by two observers $(N=15)$

\begin{tabular}{|c|c|c|c|}
\hline & Mean $(\mu \mathrm{m})$ & $\begin{array}{l}\text { Intra-obserser } \\
\text { precision }(\mu \mathrm{m})\end{array}$ & $\begin{array}{l}\text { Coefficient of } \\
\text { variation (\%) }\end{array}$ \\
\hline & \multicolumn{3}{|c|}{ Entire artery segment } \\
\hline \multicolumn{4}{|l|}{ Adventitia-to-adventitia } \\
\hline End-diastolic diameters & $8555 \pm 756$ & 289 & 3 \\
\hline Distension & $231 \pm 153$ & 84 & 39 \\
\hline \multicolumn{4}{|l|}{ Lumen } \\
\hline End-diastolic diameters & $5547 \pm 1066$ & 278 & 5 \\
\hline \multirow[t]{2}{*}{ Distension } & $234 \pm 163$ & 95 & 41 \\
\hline & \multicolumn{3}{|c|}{ At maximal posterior plaque thickness } \\
\hline \multicolumn{4}{|l|}{ Adventitia-to-adventitia } \\
\hline End-diastolic diameters & $8648 \pm 885$ & 330 & 4 \\
\hline Distension & $189 \pm 158$ & 76 & 40 \\
\hline \multicolumn{4}{|l|}{ Lumen } \\
\hline End-diastolic diameters & $4218 \pm 1036$ & 183 & 4 \\
\hline Distension & $222 \pm 159$ & 112 & 50 \\
\hline Plaque compression & $-6 \pm 130$ & 146 & 2433 \\
\hline
\end{tabular}

Orthogonal adventitia-adventitia and lumen diameters can be determined with good inter-observer precision, whereas the precision of distension is poor. However, inter-observer precision of plaque compression is larger than the mean compression and is of the same order as the standard deviation of plaque compression of our patient population. Therefore, plaque compression cannot be precisely determined. Values are presented as the mean \pm standard deviation. Inter-observer precision is defined as the standard deviation of differences between measurements and their average of all observers.

Table 5.4. Lumen and adventitia-adventitia diameters and distensions at three locations about the plaque site $(N=14)$

\begin{tabular}{|c|c|c|c|c|c|c|}
\hline & \multirow[b]{2}{*}{$\begin{array}{c}\text { Proximal } \\
(\mu \mathrm{m})\end{array}$} & \multirow[b]{2}{*}{$\begin{array}{c}\text { Plaque } \\
(\mu \mathrm{m})\end{array}$} & \multirow[b]{2}{*}{$\begin{array}{l}\text { Distal } \\
(\mu \mathrm{m})\end{array}$} & \multicolumn{3}{|c|}{$p$ value } \\
\hline & & & & 1 & 2 & 3 \\
\hline \multicolumn{7}{|l|}{ Diameter } \\
\hline Adventitia-adventitia & $7826 \pm 863$ & $8237 \pm 842$ & $8419 \pm 843$ & 0.009 & 0.087 & 0.23 \\
\hline Lumen & $6233 \pm 1195$ & $4689 \pm 1071$ & $6592 \pm 1098$ & 0.001 & $<0.001$ & $<0.001$ \\
\hline \multicolumn{7}{|l|}{ Distension } \\
\hline Adventitia-adventitia & $194 \pm 161$ & $216 \pm 161$ & $286 \pm 183$ & 0.49 & 0.15 & 0.46 \\
\hline Lumen & $311 \pm 196$ & $294 \pm 211$ & $351 \pm 235$ & 0.71 & 0.27 & 0.40 \\
\hline
\end{tabular}

Though the adventitia-adventitia diameter was larger at maximal plaque thickness than at the common carotid artery proximal to the plaque ( $p$ value 1), suggesting outward remodeling, the difference vanished for the distal diameter ( $p$ value 2 ) or for the mean of both proximal and distal diameters ( $p$ value 3). No spatial differences were observed for the local distensions. 


\section{Orthogonal and vertical lumen diameters}

The mean orthogonal lumen diameter along the plaque segment (smallest $40 \%$ ) of the 23 patients is $4720 \pm 1176 \mu \mathrm{m}$, whereas the lumen diameter along the ultrasound beam is $5344 \pm 1094 \mu \mathrm{m}$. Therefore, the orthogonal lumen diameter is significantly smaller than the vertical diameter (mean difference: $625 \mu \mathrm{m}, p<0.001$ ) and reflects more properly local lumen narrowing, corroborating the importance of orthogonal measurements.

\section{Dynamic parameters at the CCA with and without plaque}

Fourteen of the 23 patients had a mean posterior wall thickness $<1500 \mu \mathrm{m}$ proximal as well as distal to the largest plaque. As expected, the end-diastolic orthogonal lumen diameter at maximal plaque thickness is significantly lower than that proximal or distal to the plaque (mean differences: 1544 and $1903 \mu \mathrm{m}, p=0.001$ and $p<0.001$, respectively) (Table 5.4). The end-diastolic adventitia-adventitia diameter is significantly larger at the plaque than at the proximal segment (mean difference: $411 \mu \mathrm{m}, p=0.009$ ), suggesting outward remodeling. However, the end-diastolic adventitia-adventitia diameter at the CCA distal to the plaque is even borderline significantly larger than that at the site of the plaque (mean difference: $183 \mu \mathrm{m} ; p=0.087$ ), which is in line with a diverging CCA toward the bifurcation and contradicts outward remodeling. Indeed, the plaque adventitia-adventitia diameter does not deviate from the mean of the proximal and distal adventitia-adventitia diameters $(p=0.23)$ (Table 5.4). The adventitia-adventitia and lumen distensions at the plaque are similar to either the distension at the CCA proximal or distal to the plaque (mean difference: $<70 \mu \mathrm{m}, p>0.15$ ).

\section{Dynamic parameters and grey-scale values}

Figure $\mathbf{5 . 4}$ shows scatterplots of the relative adventitia-adventitia and lumen distensions at the site of maximal plaque thickness $(\mathrm{N}=23)$ compared with the $75^{\text {th }}$ percentile of the normalized grey-scale value (GS75). The relative adventitia-adventitia and lumen distensions are negatively correlated to the GS75 (Pearson's correlations $r=-0.35, p=$ 0.097 and $r=-0.50, p=0.015$, respectively). The group median of the GS75 is 72 on the normalized 0-199 scale. Patients with a GS75 higher than $72(\mathrm{~N}=11)$ exhibit a (borderline) significantly lower relative adventitia-adventitia and lumen distensions ( $p$ values $=0.084$ and 0.009 , respectively), indicating higher vessel wall stiffness. 

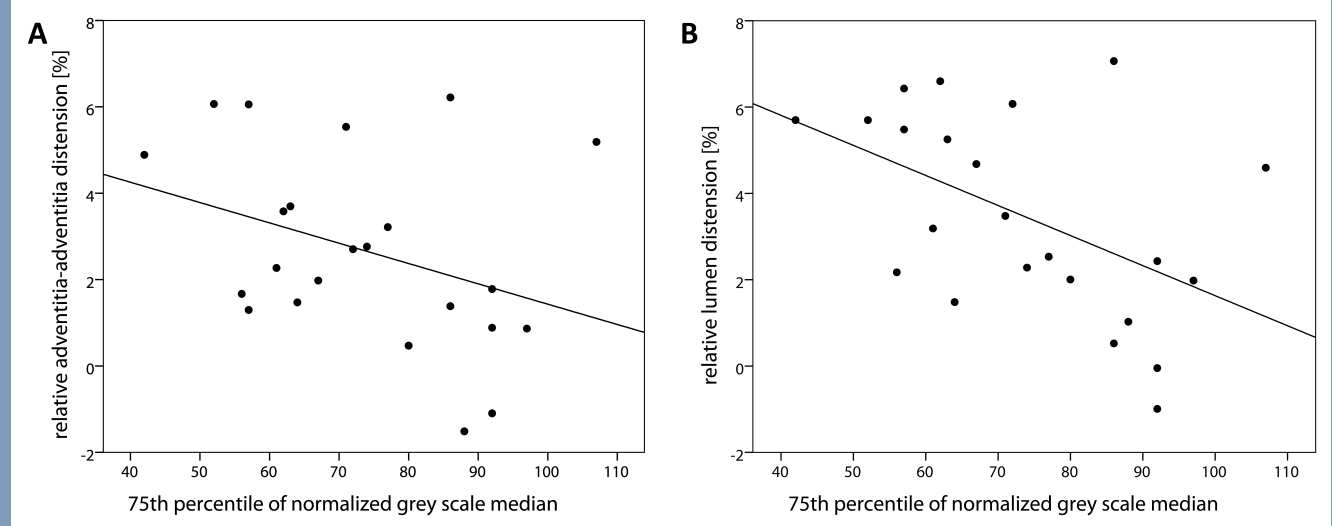

Figure 5.4. Orthogonal relative adventitia-adventitia distension (A) and lumen distension (B) at the site of maximal plaque thickness are negatively correlated with the $75^{\text {th }}$ percentile of the grey-scale value (Pearson correlation of -0.35 and -0.50 , with p-values of 0.097 and 0.015 , respectively. 


\section{Discussion}

The present study explored the option to extract from ultrasound images the orthogonal dimensions of the CCA and plaques, that is, the dimensions perpendicular to the local orientation of the artery centerline. Orthogonal adventitia-adventitia and lumen diameters could be determined with good precision, whereas the precision of distension is poor. At the site of the plaque, the orthogonal lumen diameter differs substantially from that measured along the ultrasound beam (vertical direction for B-mode image obtained with a linear array). Because the intra-observer precision and inter-observer precision of plaque compression are of the same order as the standard deviation of the plaque compression of the patient population, plaque compression cannot be precisely determined. Contrary to the general concept of outward remodeling (induced by the locally increased wall shear strain) at the site of the plaque, we observed a gradually increasing orthogonal adventitia-adventitia diameter toward the CCA bifurcation. Furthermore, orthogonal relative adventitia-adventitia and lumen distensions at the site of maximal plaque thickness are negatively associated with the $75^{\text {th }}$ percentile of the normalized grey-scale value.

As expected, the observed intra-subject precision of the orthogonal adventitiaadventitia diameter is low (CV: 1\%) and similar to values reported in the literature (CV: 1.5\%) without correction for the angle of interrogation..$^{18}$ The observed intra-subject precision of orthogonal distension $(40 \mu \mathrm{m})$ is also similar to the CCA distension $(44 \mu \mathrm{m})$ of patients with plaques in the internal carotid artery ${ }^{18}$ and of the subgroups of Graf et al. ${ }^{21}$ with plaques in the ipsilateral or contralateral common or internal carotid artery. Note that we used the average of repeated measurements as reference for calculating the intra-subject precision, whereas Graf et al. used one measurement as reference for the other, resulting in an intra-subject precision twice as high as in our current approach. However, because of a smaller mean distension $(193 \pm 128 \mu \mathrm{m})$ as a consequence of the plaque (Table 5.2), the CV is substantially larger (21\%) than reported values determined in a CCA without plaque (mean distension: $377 \pm 122 \mu \mathrm{m}, \mathrm{CV}: 12 \%$ ). ${ }^{18}$ The mean adventitiaadventitia distension $(193 \mu \mathrm{m})$ is also substantially smaller than reported $(>300 \mu \mathrm{m})$ by others for common carotid artery plaques., 7,8 This is explained by the fact that we considered the orthogonal distension along the entire segment rather than the proximal value only, where pulse pressures are higher because of pulse wave reflection. ${ }^{22}$ On the other hand, within the stenosis, the transmural pressure will be reduced..$^{22}$

The intra-observer CVs of the orthogonal lumen diameter and distension are smaller than the inter-observer CVs. No specific rules were issued regarding the segmentation, allowing free interpretation of the observer and explaining larger inter-observer variations. The intra- and inter-observer CVs of lumen diameter ( $2 \%$ and $5 \%$ ) and distension ( $28 \%$ and $41 \%$ ) are larger than the CVs of adventitia-adventitia diameter ( $1 \%$ and $3 \%$ ) and distension (21\% and 39\%). This is likely due to the problems associated with the determination of the 
lumen-intima transition at the near wall and at oblique observation angles. Accordingly, the Mannheim consensus recommends assessment of intima-media thickness at the far wall only, instead of near and far wall. ${ }^{23}$ Because both intra-observer precision and inter-observer precision of adventitia-adventitia and lumen diameters and distensions are substantially smaller than the observed standard deviation of those parameters in our patient population, orthogonal diameter and distension can be precisely determined from ultrasound images.

In contrast to the distension and diameter, orthogonal plaque compression at the site of maximal plaque thickness cannot be precisely determined. This is because the intra- and inter-observer precisions of plaque compression are higher than the actual compression and are of the same order as the standard deviation of compression in our patient population. The main reason for the high intra-and inter-observer variability is the relatively poor ultrasound resolution of $300 \mu \mathrm{m}$ compared with the small variation in plaque thickness. ${ }^{24}$ Because we considered the parameters at the location of maximal plaque thickness, axial artery/plaque displacements across images are automatically corrected for. The variability cannot be adequately improved by averaging over subsequent heartbeats. Another option is to consider spatial averaging over the plaque. However, plaque compression may vary along the plaque because of spatial variations in plaque thickness, composition and local transmural pulse pressure.

In this study, we explored the possibility of extracting the CCA dimensions and plaque thickness perpendicular to the local orientation of either the adventitia-adventitia or lumen centerline. Because the CCA is commonly parallel to the skin surface, the orthogonal artery dimensions for segments without a plaque do not substantially deviate from those obtained with standard procedures. ${ }^{18,21}$ However, at a stenotic segment, the artery orientation will change with position along the stenotic segment (Figure 5.1). Consequently, the orthogonal dimensions at the proximal and distal plaque segments are lower than those observed along the ultrasound beam. For artery segments with an oblique orientation (e.g. the ICA), larger differences between orthogonal and perpendicular assessment may occur.

As stated earlier, the presence of a stenosis will locally increase the blood flow velocity and, hence, the wall shear stress. This will stimulate the endothelium to release a vasodilating agent $(\mathrm{NO})$ in an attempt to restore the local wall shear stress. ${ }^{4-6}$ Consequently, the adventitia-adventitia diameter should be larger at the site of a stenosis (outward remodeling). To investigate this effect, we considered the adventitia-adventitia and lumen diameters and distensions proximal and distal to a plaque in a subset ( $\mathrm{N}$ $=14$ ). Because of the stenosis, the lumen diameter at maximal plaque thickness is significantly smaller than the corresponding lumen diameter either proximal or distal to the plaque (Table 5.4). On the other hand, the adventitia-adventitia diameter at maximal plaque thickness is significantly larger than the diameter proximal to the plaque (Table 5.4), suggesting outward remodeling. This is in line with studies in which outward 
remodeling was observed for a subset of the CCA plaques considered. ${ }^{2,7,8}$ However, the adventitia-adventitia diameter at the plaque was borderline significantly smaller than the diameter distal to the plaque (Table 5.4), whereas there was no difference with the mean of proximal and distal diameters. Those observations are in line with a diverging CCA toward the bifurcation and contradict the concept of outward remodeling. Because CCA plaques are relatively stable compared with plaques at the downstream bifurcation, outward remodeling may indeed be limited, even though the average stenosis degree in our population was $48 \%$ (Table 5.1). In addition, it should be realized that proximal or distal to an observed segment, there may exist plaques that affect the parameters extracted from the recording.

Orthogonal adventitia-adventitia and lumen relative distensions at the site of maximal plaque thickness are negatively associated with the GS75. Thus, a locally stiffer vessel wall, as reflected by a decreased relative lumen distension, is associated with echogenic plaques probably caused by a higher calcium content.9, 10 Lumen distension always exceeds adventitia-adventitia distension, and changes caused by a (locally) deviating wall stiffness will inherently have a larger effect on lumen distension. The present study was performed in a relatively small cohort $(N=23)$, which further explains the weak association between adventitia-adventitia distension and GS75.

A limitation of this study is the relative stability of CCA plaques; CCA plaques will almost never lead to rupture. However, CCA plaque evaluation will contribute to the knowledge of plaque dynamics and relation with plaque morphology and composition. The association between the relative adventitia-adventitia and lumen distensions at the plaque within the carotid bulb and plaque progression and/or rupture risk will be investigated in the PARISK follow-up study. ${ }^{17}$ 


\section{Conclusion}

We illustrated the feasibility of extracting from a sequence of ultrasound B-mode frames the orthogonal dynamic dimensions of the common carotid artery and plaques, albeit that the proposed approach appears inadequate to establish plaque compression. At the site of the plaque, the orthogonal lumen diameter is significantly smaller than the lumen diameter along the ultrasound beam. Surprisingly, we did not find outward adventitiaadventitia remodeling at the site of CCA plaques. Orthogonal relative lumen distension is negatively associated with plaque echogenicity, which is in line with the known larger stiffness of lesions with increased echogenicity.

\section{Acknowledgments}

This research was performed within the framework of the Center for Translational Molecular Medicine (www.ctmm.nl) project PARISK (Plaque at Risk, Grant 01 C-202) and supported by the Dutch Heart Foundation (DHF-2008-T094). 


\section{References}

1. Giannattasio C, Failla M, Emanuelli G, Grappiolo A, Boffi L, Corsi D, et al. Local effects of atherosclerotic plaque on arterial distensibility. Hypertension. 2001;38:1177-1180

2. Beaussier H, Naggara O, Calvet D, Joannides R, Guegan-Massardier E, Gerardin E, et al. Mechanical and structural characteristics of carotid plaques by combined analysis with echotracking system and MR imaging. JACC Cardiovascular Imaging. 2011;4:468-477

3. Nichols WW, O'Rourke MF, Vlachopoulos C. Mcdonald's blood flow in arteries: Theoretic, experimental, and clinical principles. London: Hodder Arnold; 2011.

4. Dammers R, Stifft F, Tordoir JH, Hameleers JM, Hoeks AP, Kitslaar PJ. Shear stress depends on vascular territory: Comparison between common carotid and brachial artery. Journal of Applied Physiology (1985). 2003;94:485-489

5. Glagov S, Bassiouny HS, Sakaguchi Y, Goudet CA, Vito RP. Mechanical determinants of plaque modeling, remodeling and disruption. Atherosclerosis. 1997;131 Suppl:S13-14

6. Samijo SK, Willigers JM, Barkhuysen R, Kitslaar PJ, Reneman RS, Brands PJ, et al. Wall shear stress in the human common carotid artery as function of age and gender. Cardiovascular Research. 1998;39:515-522

7. Beaussier H, Masson I, Collin C, Bozec E, Laloux B, Calvet D, et al. Carotid plaque, arterial stiffness gradient, and remodeling in hypertension. Hypertension. 2008;52:729-736

8. Paini A, Boutouyrie P, Calvet D, Zidi M, Agabiti-Rosei E, Laurent S. Multiaxial mechanical characteristics of carotid plaque: Analysis by multiarray echotracking system. Stroke. 2007;38:117-123

9. Elatrozy T, Nicolaides A, Tegos T, Griffin M. The objective characterisation of ultrasonic carotid plaque features. European journal of vascular and endovascular surgery. 1998;16:223-230

10. Kakkos SK, Stevens JM, Nicolaides AN, Kyriacou E, Pattichis CS, Geroulakos G, et al. Texture analysis of ultrasonic images of symptomatic carotid plaques can identify those plaques associated with ipsilateral embolic brain infarction. European journal of vascular and endovascular surgery. 2007;33:422-429

11. Biasi GM, Sampaolo A, Mingazzini P, De Amicis P, El-Barghouty N, Nicolaides AN. Computer analysis of ultrasonic plaque echolucency in identifying high risk carotid bifurcation lesions. European journal of vascular and endovascular surgery. 1999;17:476-479

12. Gronholdt ML, Nordestgaard BG, Schroeder TV, Vorstrup S, Sillesen H. Ultrasonic echolucent carotid plaques predict future strokes. Circulation. 2001;104:68-73

13. Mathiesen EB, Bonaa $\mathrm{KH}$, Joakimsen $\mathrm{O}$. Echolucent plaques are associated with high risk of ischemic cerebrovascular events in carotid stenosis: The Tromso study. Circulation. 2001;103:2171-2175

14. Topakian R, King A, Kwon SU, Schaafsma A, Shipley M, Markus HS, et al. Ultrasonic plaque echolucency and emboli signals predict stroke in asymptomatic carotid stenosis. Neurology. 2011;77:751-758

15. Salem MK, Sayers RD, Bown MJ, West K, Moore D, Nicolaides A, et al. Patients with recurrent ischaemic events from carotid artery disease have a large lipid core and low GSM. European journal of vascular and endovascular surgery. 2012;43:147-153

16. Martinez-Sanchez P, Fernandez-Dominguez J, Ruiz-Ares G, Fuentes B, Alexandrov AV, Diez-Tejedor E. Changes in carotid plaque echogenicity with time since the stroke onset: An early marker of plaque remodeling? Ultrasound in medicine \& biology. 2012;38:231-237

17. Truijman MT, Kooi ME, van Dijk AC, de Rotte AA, van der Kolk AG, Liem MI, et al. Plaque at risk 
(PARISK): Prospective multicenter study to improve diagnosis of high-risk carotid plaques. International journal of stroke. 2014;9:747-754

18. Steinbuch J, Hoeks AP, Hermeling E, Truijman MT, Schreuder FH, Mess WH. Standard B-mode ultrasound measures local carotid artery characteristics as reliably as radiofrequency phase tracking in symptomatic carotid artery patients. Ultrasound in medicine \& biology. 2016;42:586-595

19. Steinbuch J, van Dijk AC, Schreuder F, Truijman MTB, de Rotte AAJ, Nederkoorn PJ, et al. High spatial inhomogeneity in the intima-media thickness of the common carotid artery is associated with a larger degree of stenosis in the internal carotid artery: The PARISK study. Ultraschall in der Medizin. 2017;38:523-529

20. Randomised trial of endarterectomy for recently symptomatic carotid stenosis: Final results of the MRC European carotid surgery trial (ECST). Lancet. 1998;351:1379-1387

21. Graf IM, Schreuder FH, Mess WH, Reneman RS, Hoeks AP. Spatial distension variations are associated with focal atherosclerotic plaques. Cerebrovascular diseases. 2010;29:199-205

22. Hoeks AP, Reesink KD, Hermeling E, Reneman RS. Local blood pressure rather than shear stress should be blamed for plaque rupture. Journal of the American College of Cardiology. 2008;52:11071108; author reply 1108-1109

23. Touboul PJ, Hennerici MG, Meairs S, Adams H, Amarenco P, Bornstein N, et al. Mannheim carotid intima-media thickness and plaque consensus (2004-2006-2011). An update on behalf of the advisory board of the 3rd, 4th and 5th watching the risk symposia, at the 13th, 15th and 20th European stroke conferences, Mannheim, Germany, 2004, Brussels, Belgium, 2006, and Hamburg, Germany, 2011. Cerebrovascular diseases. 2012;34:290-296

24. Steinbuch J, van Dijk AC, Schreuder F, Truijman M, Hendrikse J, Nederkoorn PJ, et al. Definition of common carotid wall thickness affects risk classification in relation to degree of internal carotid artery stenosis: The plaque at risk (PARISK) study. Cardiovascular Ultrasound. 2017;15:9 




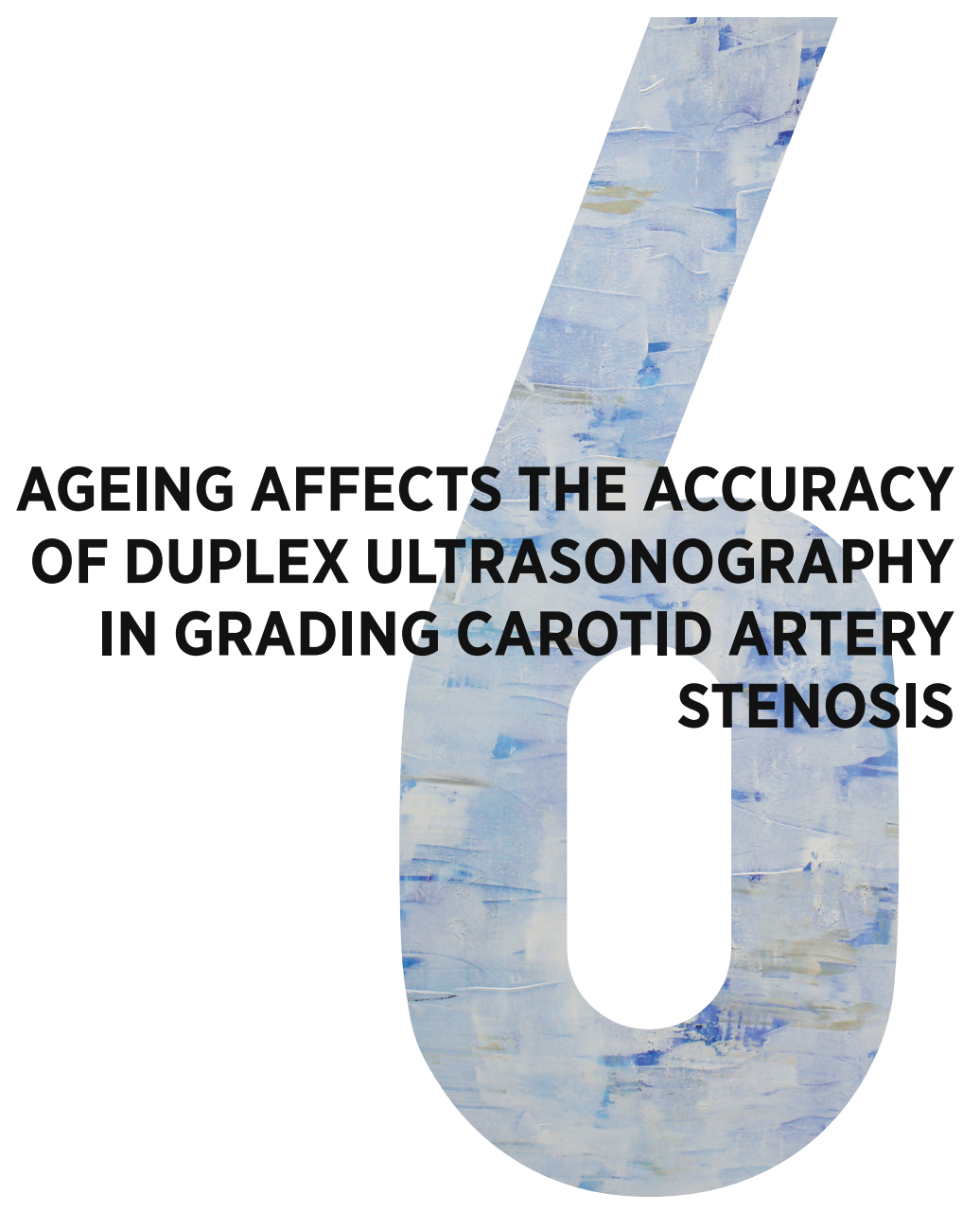

FHBM Schreuder, WH Mess, APG Hoeks

Published in:

Cerebrovascular Diseases 2009; 27(1): 75-83 


\section{Abstract}

Background: Duplex ultrasonography is frequently used to select patients who are eligible for carotid endarterectomy by using local blood flow velocities to quantify the degree of internal carotid artery stenosis. However, ageing affects flow velocities and might hence influence the accuracy of duplex ultrasonography, as explored in the present study.

Methods: We determined peak-systolic (PSV) and end-diastolic (EDV) blood flow velocities in the common carotid artery (CCA) and internal carotid artery (ICA) in the absence of atherosclerosis and correlated these with age. The established correlation was applied to measurements in the ICA with visualized atherosclerosis to evaluate whether an age-related change in blood flow velocities modified stenosis grading.

Results: In 465 arteries without atherosclerosis, PSV declined linearly in both the CCA ( $r=$ $-0.55)$ and ICA $(r=-0.44)$. The EDV decreased linearly in the CCA and ICA $(r=-0.61$ and $r=-0.56$, respectively). In 774 internal carotid arteries with atherosclerosis present, the use of an age-dependent reference increased the estimated degree of stenosis in 3 young subjects. The stenosis grade was reduced in 11 older subjects, mainly in symptomatic arteries.

Conclusions: Blood flow velocities in the carotid artery system decrease continuously with increasing age. This may affect the estimated degree of internal carotid artery stenosis in the elderly. 


\section{Introduction}

Severe stenosis of the internal carotid artery (ICA) may be the source of atherothrombotic embolism leading to ischaemic stroke. Hence, patients with more than 70\% ICA stenosis established by angiography benefit from carotid endarterectomy, as was demonstrated for example by the pooled data from the North American Symptomatic Carotid Endarterectomy Trial (NASCET) and the European Carotid Surgery Trial (ECST) ${ }^{1-3}$ Carotid surgery has also been shown to be beneficial in designated subjects with high-grade asymptomatic stenosis., ${ }^{4}$

Nowadays, carotid angiography is frequently replaced by non-invasive diagnostic methods like duplex ultrasonography. ${ }^{6,7}$ Duplex ultrasonography utilizes quantitative Doppler measurements of blood flow velocities throughout the carotid system to determine the degree of stenosis. ${ }^{8}$ However, discussion remains on the definite cut-off points for peak-systolic (PSV) and end-diastolic velocities (EDV) to determine a given degree of stenosis; a well-defined threshold is essential for the selection of patients who are eligible for carotid endarterectomy. ${ }^{9-12}$

Moreover, increasing age might also affect the diagnostic accuracy of duplex ultrasonography. Ageing is known to influence both structural and functional vascular properties..$^{13-16}$ Increasing age affects these vascular properties independently of increased exposure to the traditional well-known cardiovascular risk factors (e.g. smoking, hypertension, diabetes mellitus, hypercholesterolemia).17, 18 Changes in structural and functional properties result in an age-dependent decline in blood flow velocity, as was previously shown for the common carotid artery $(C C A)^{19-24}$ and the internal carotid artery. ${ }^{21-24}$ Since duplex ultrasonography uses local blood flow velocity to establish the degree of stenosis, an age-related decline might influence the accuracy of grading carotid artery stenosis for the lower and upper age categories.

The purpose of the present study is twofold. First, we aim to confirm the effects of ageing on blood flow velocities in the CCA and the ICA of subjects without overt carotid atherosclerotic lesions present, defined as focal increased wall thickness or plaque formation in the CCA and ICA observed with B-mode ultrasound imaging. Secondly, we will evaluate whether age influences the accuracy of duplex ultrasonography in determining the degree of ICA stenosis in subjects with confirmed plaque formation in the carotid artery. 


\section{Methods}

\section{Study subjects}

We carried out a retrospective analysis of all carotid duplex ultrasonography studies ( $n=$ 920) at our department between January and December 2006.

Eight patients with unilateral or bilateral occlusion of the CCA were excluded from analysis since determination of blood flow velocities was not possible. More than 1 duplex ultrasonography examination (range 2-4) was performed in 67 patients. In these patients, the following criteria were used to select the duplex study that was used for further analysis: (1) in case of multiple ischaemic events, the latest duplex ultrasonography study was included; (2) in patients who underwent carotid endarterectomy in 2006, and thus required several ultrasonography studies, the pre-operative duplex study was included. Also, 21 patients were excluded because of carotid surgery in previous years, which could introduce bias due to the modified vascular morphology as a result of treatment. Altogether, a total of 810 patients were included in this study (mean age $=66.7 \pm 13.2$ years, range $=14-94$ years, $53.5 \%$ male). The indications for duplex ultrasonography are listed in Table 6.1.

Table 6.1. Indications for carotid ultrasonography $(n=810)$.

\begin{tabular}{lcc} 
Indication & N & $\%$ \\
Cerebrovascular ischemia & 601 & 74.2 \\
Stroke & 332 & 41.0 \\
Transient ischemic attack & 227 & 28.0 \\
$\quad$ Retinal ischemia & 42 & 5.2 \\
\hline Pre-operative carotid endarterectomy a $^{\text {a }}$ & 46 & 5.7 \\
\hline Asymptomatic $^{\text {b }}$ & 149 & 18.4 \\
\hline Dissection $^{c}$ & 14 & 1.7 \\
\hline
\end{tabular}

a In total, 44 out of 46 patients had cerebrovascular ischemia within 6 months prior to carotid endarterectomy.

$b$ Asymptomatic patients were referred to our department for indications other than symptoms of cerebrovascular ischemia (e.g. pre-operative screening before coronary artery bypass grafting or general screening for atherosclerosis.

c Clinical signs of dissection without cerebrovascular ischemia.

\section{Duplex ultrasonography protocol}

Ultrasonography was performed by a well-trained member of staff using a color-coded duplex machine (SONOS 5500, Philips Medical Systems, Eindhoven, The Netherlands) equipped with a 3-11 MHz linear-array transducer. The investigations were performed in a supine position with the head tilted slightly to the opposite side. In all patients, the left and right CCA, ICA, external carotid artery and vertebral artery were examined, but for the present study only the Doppler blood flow velocity measurements of the CCA and ICA were taken into account. 
Enlarged intima-media thickness and local plaque of the CCA, as seen in the B-mode view, were listed according to the Mannheim Consensus. ${ }^{25}$ In addition, the presence of plaque formation at the carotid bulb or ICA was noted. The PSV and EDV were measured using color-assisted Doppler mode and selecting the highest velocities. Blood flow velocity was assessed in the distal CCA, approximately $1 \mathrm{~cm}$ proximal to the carotid bifurcation. In the ICA, PSV and EDV were measured at 3 sites: at the proximal (approximately $1 \mathrm{~cm}$ from flow divider) and medial ICA (approximately $3 \mathrm{~cm}$ from flow divider) and as far distal as possible. In the absence of any atherosclerotic lesions, proximal measurements were used, whereas the highest PSV and EDV were used for analysis in case of atherosclerotic plaque.

To determine the degree of stenosis, the criteria as outlined by the Society of Radiologists in Ultrasound were employed." These criteria define the absolute cut-off references for $50 \%$ and $70 \%$ stenosis as ICA PSV of $125 \mathrm{~cm} / \mathrm{s}$ and $230 \mathrm{~cm} / \mathrm{s}$, respectively. Near-total occlusion was defined as PSV $<100 \mathrm{~cm} / \mathrm{s}$ at the site of a severe atherosclerotic lumen reduction resulting in 'trickle flow' at optimal color gain settings. Total occlusion was determined by the absence of detectable blood flow within the vessel in conjunction with detection of a 'to-and-fro' signal directly proximal to the occluded segment.

\section{Analysis}

To avoid within-patient clustering, separate analyses were carried out for the symptomatic and asymptomatic arteries. In subjects without cerebral ischemia $(n=165$; Table 6.1), only 1 artery was randomly selected and analyzed in the asymptomatic subgroup. In subjects with cerebral ischemia ( $n=645$; Table 6.1), the symptomatic artery was analyzed in the symptomatic subgroup, whereas the contralateral artery was included into the asymptomatic cohort. Hence, we could analyze 810 asymptomatic arteries and 645 symptomatic arteries (a total of 1,455 arteries).

To investigate the effect of age on absolute blood flow velocities, subjects with an absence of atherosclerotic lesions in both the left and right CCA and ICA were selected, thereby excluding the confounding effect of ipsilateral elevated blood flow velocities because of the contralateral presence of atherosclerosis. ${ }^{26}$ For symptomatic $(n=181$ arteries) and asymptomatic ( $n=284$ arteries) arteries separately, and for both groups together ( $n=465$ arteries), linear regression analysis on the relation between age and PSV or EDV in both the CCA and ICA was performed. Regression coefficients (intercept and slope) are expressed with their 95\% confidence intervals $(\mathrm{Cl})$. Pearson correlation coefficient (Pearson's $r$ ) was calculated to determine the degree of correlation. Subjects were divided into quartiles according to age in order to study the relative decrease in blood flow velocity with increasing age.

In the second part of the study, the effect of age on the accuracy of duplex ultrasonography was analyzed. The remaining arteries with atherosclerosis at the ICA were selected, i.e. arteries without atherosclerosis $(n=156)$ were excluded. In addition, 
60 arteries with total or near-total occlusion at the ICA were excluded from analysis since in these cases measurement of blood flow velocities is inadequate for determining the degree of stenosis. In total, 410 asymptomatic and 364 symptomatic arteries were selected $(n=774)$. Table 6.2 summarizes the degree of stenosis in the symptomatic and asymptomatic arteries according to the criteria as proposed by Grant et al."

A scatter plot of the PSV in the ICA was obtained, and related to the absolute cutoff references for $50 \%$ stenosis (PSV $=125 \mathrm{~cm} / \mathrm{s}$ ) and $70 \%$ stenosis $(P S V=230 \mathrm{~cm} / \mathrm{s}$ ). For both the symptomatic and asymptomatic arteries, the slope of the PSV in the ICA in the non-atherosclerotic, matching subpopulation was used to establish age-dependent reference lines for both 50 and $70 \%$ stenosis of the ICA. The point of junction between the absolute and age-dependent reference lines was chosen according to the mean age (64 years) of the patients described in the pooled data from the NASCET and ECST trials by Rothwell et al.' Using this point of junction, the intercepts were recalculated for the 2 age-dependent reference lines with their $95 \%-\mathrm{Cl}$.

Age data is presented as means $\pm \mathrm{SD}$, and all other data as point estimates with $95 \%$ $\mathrm{Cl}$. The significance level was set at $p<0.05$. All statistical analyses were carried out using SPSS for Windows (version 14.0).

Table 6.2. Degree of stenosis amongst 810 asymptomatic and 645 symptomatic internal carotid artery (ICA) established by duplex ultrasonography according to the criteria by Grant et al."

\begin{tabular}{|c|c|c|c|c|}
\hline \multirow[t]{2}{*}{ Degree of stenosis } & \multicolumn{2}{|c|}{ Asymptomatic } & \multicolumn{2}{|c|}{ Symptomatic } \\
\hline & $\mathbf{N}$ & $\%$ & $\mathbf{N}$ & $\%$ \\
\hline No carotid artery disease ${ }^{a}$ & 386 & 47.6 & 235 & 36.4 \\
\hline Less than $50 \%$ stenosis & 322 & 41.0 & 229 & 35.5 \\
\hline $50-69 \%$ stenosis & 58 & 7.2 & 49 & 7.6 \\
\hline $70-99 \%$ stenosis & 20 & 2.5 & 86 & 13.3 \\
\hline Near-total occlusion & 2 & 0.2 & 6 & 0.9 \\
\hline Occlusion & 12 & 1.5 & 40 & 6.2 \\
\hline
\end{tabular}

${ }^{a}$ No present plaque formation or stenosis at the ICA. 


\section{Results}

Influence of age on blood flow velocity in subjects with no carotid atherosclerosis In total, 465 subjects were considered to be free of atherosclerotic disease (mean age $=59.5 \pm 14.7$ years, range $=14-89$ years, $48.0 \%$ male). Table 6.3 illustrates the effect of ageing on the PSV and EDV in the ICA and CCA for both symptomatic and asymptomatic arteries, as well as for the whole group. Table 6.4 compares the effect of ageing as determined in the present study with previously published literature on this subject. All blood flow velocities significantly declined linearly with increasing age (Figure 6.1; for all $p<0.001$ ). For the symptomatic arteries, the PSV in the ICA declined by $21.1 \%$ between the youngest and oldest age quartiles with an intercept of $87.98 \mathrm{~cm} / \mathrm{s}(95 \%-\mathrm{Cl}=77.45-98.51$ $\mathrm{cm} / \mathrm{s})$ and a slope of $-0.46 \mathrm{~cm} / \mathrm{s} /$ year $(95 \%-\mathrm{Cl}=-0.29$ to $-0.63 \mathrm{~cm} / \mathrm{s} /$ years $)$. In addition, the PSV in the asymptomatic ICA decreased by $31.2 \%$ between the youngest and oldest subgroups with a linear decline (intercept $=97.03 \mathrm{~cm} / \mathrm{s}, 95 \%-\mathrm{Cl}=89.22-104.84 \mathrm{~cm} / \mathrm{s}$; slope $=-0.59 \mathrm{~cm} /, 95 \%-\mathrm{Cl}=-0.46$ to $-0.72 \mathrm{~cm} / \mathrm{s} /$ year $)$.

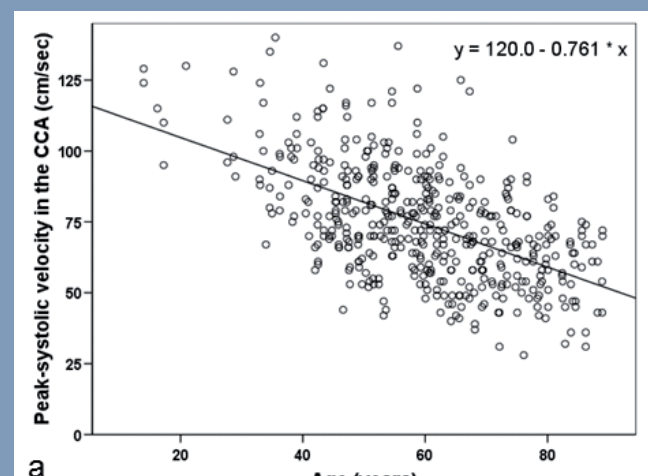

a

Age (years)
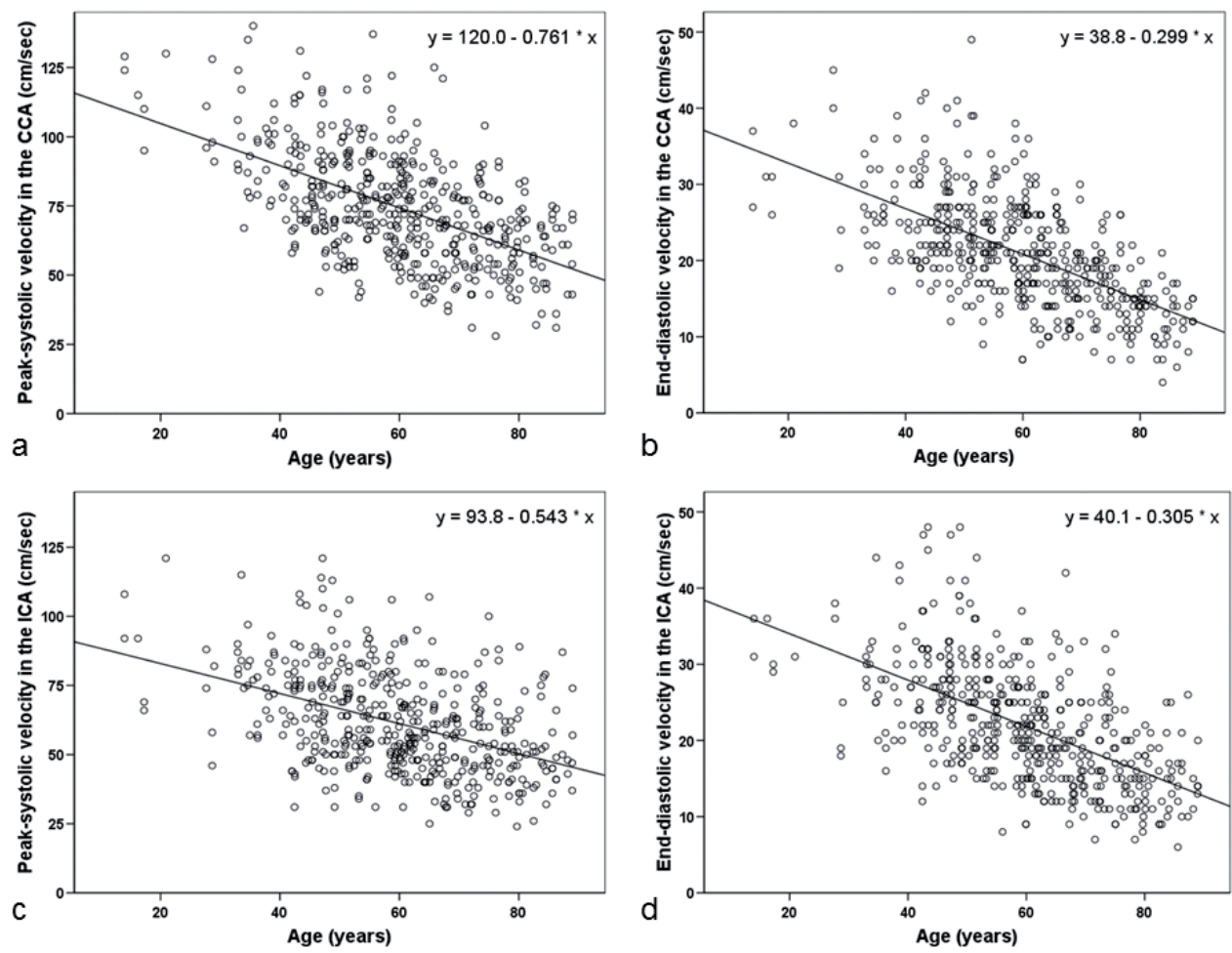

Figure 6.1. Relation between age and blood flow velocities in the common carotid (CCA) and internal carotid artery (ICA) without atherosclerosis ( $n=465$ arteries). $A$ and $C$ reflect the peak-systolic velocity (PSV; range $0-125 \mathrm{~cm} / \mathrm{s}$ ) of the CCA and ICA, respectively. $B$ and D explore the end-diastolic velocity (EDV; range $0-50 \mathrm{~cm} / \mathrm{s}$ ) of the CCA and ICA, respectively. 


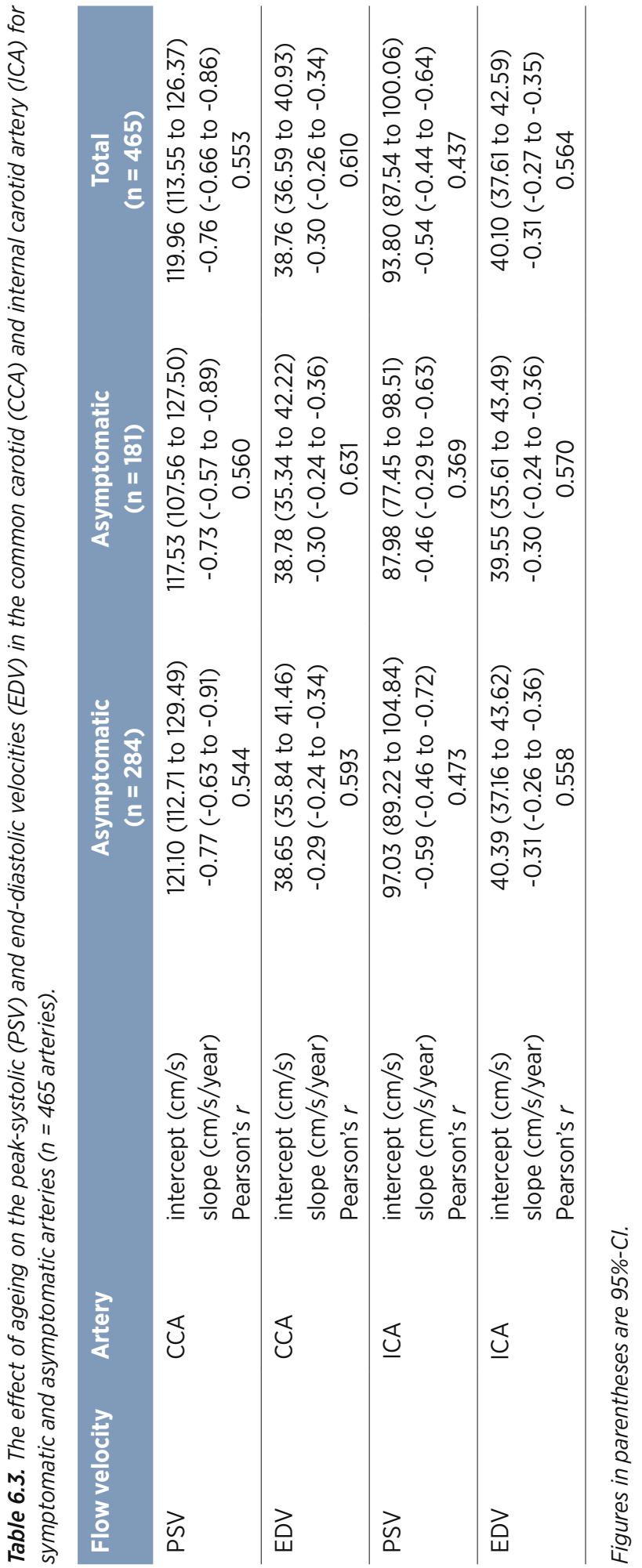




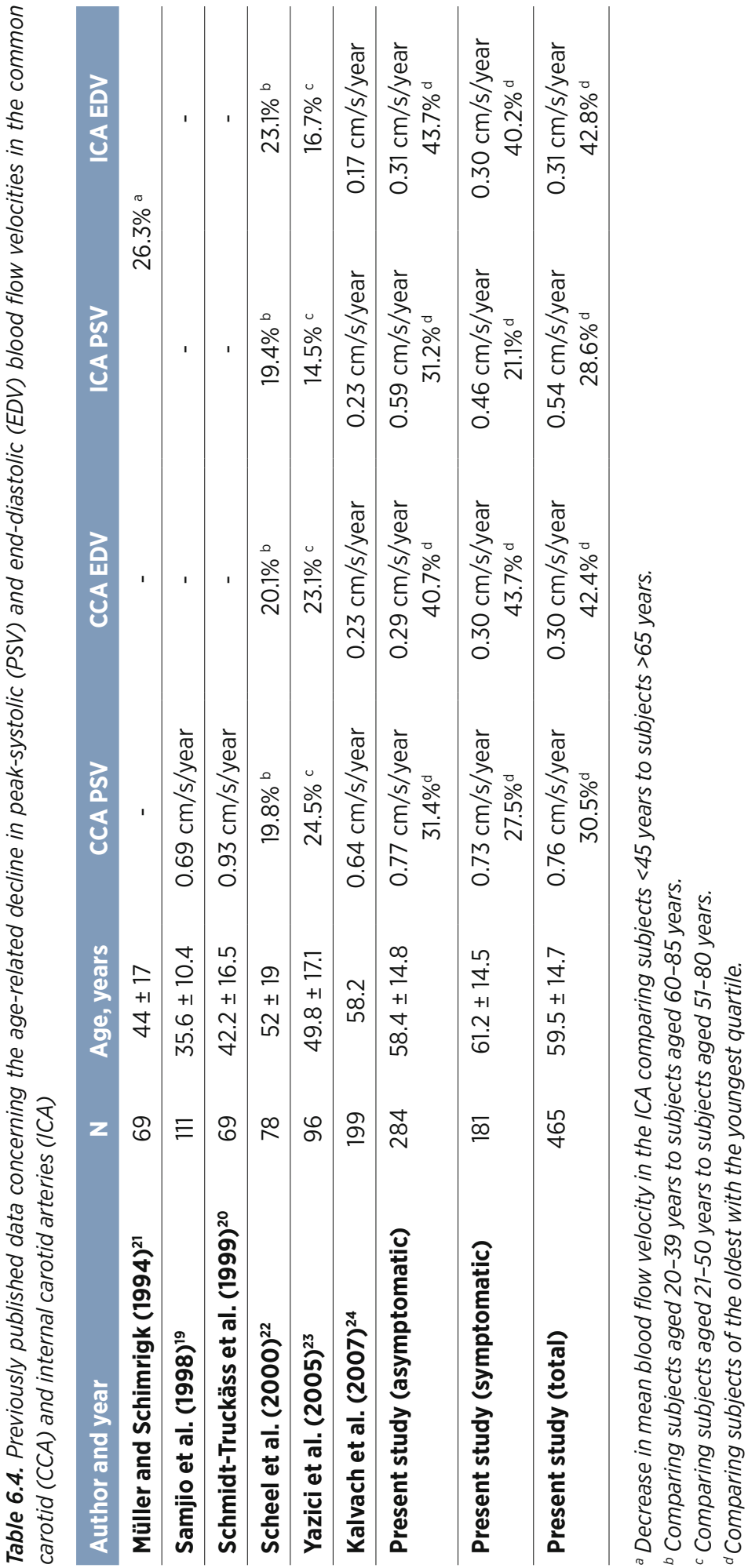


Influence of age on diagnostic accuracy of duplex ultrasonography

In total, 410 asymptomatic and 364 symptomatic subjects were used in the analysis; the mean age was not significantly different between both groups (71.4 \pm 10.1 and $72.1 \pm$ 9.6 years, respectively), and $56.4 \%$ were male. For the asymptomatic arteries, the agedependent reference line for the detection of 50\% ICA stenosis ( $y=163.0-0.59 x)$ was drawn with its $95 \%-\mathrm{Cl}(155.2$ to 170.8$)$ in the scatter plot relating PSV in the ICA and age (Figure 6.2A). For discrimination of 70\% ICA stenosis, the age-dependent reference line $(y=268.0-0.59 x)$ with its $95 \%-\mathrm{Cl}(260.2$ to 275.8$)$ are also presented in Figure 6.2A. For the symptomatic subjects, Figure $6.2 \mathrm{~B}$ contains both the age-dependent reference line for the $50 \%$ ICA stenosis ( $y=154.1-0.46 x, 95 \%-C l=143.6$ to 164.6$)$ as well as for the $70 \%$ ICA stenosis ( $y=259.1-0.46 x, 95 \%-C l=248.6$ to 269.6 ).

When operating the absolute cut-off levels, 561 patients $(72.5 \%)$ had a stenosis $<50 \%$, 107 patients (13.8\%) had 50-69\% stenosis, whereas in 106 patients (13.7\%) a stenosis of more than $70 \%$ was found. Table 6.5 compares the differences between the degree of stenosis when applying either the absolute or the age-dependent reference lines. Applying the age-dependent reference line resulted in an additional $10(95 \%-\mathrm{Cl}=1-27)$ arteries with a diagnosis of $50-69 \%$ stenosis. Mean PSV in these subjects was $117 \pm 4 \mathrm{~cm} / \mathrm{s}$ ( range $=111-124 \mathrm{~cm} / \mathrm{s})$. One $(95 \%-\mathrm{Cl}=0-7)$ artery was additionally estimated to have more than $70 \%$ stenosis (mean PSV $=223 \pm 5 \mathrm{~cm} / \mathrm{s}$, range $=215-227 \mathrm{~cm} / \mathrm{s}$ ). Eight subjects, in whom the estimated degree of stenosis increased, had recent cerebrovascular ischemia; 3 subjects were asymptomatic. In $3(95 \%-\mathrm{Cl}=1-14)$ arteries, the estimated degree of stenosis decreased by the use of the absolute cut-off.
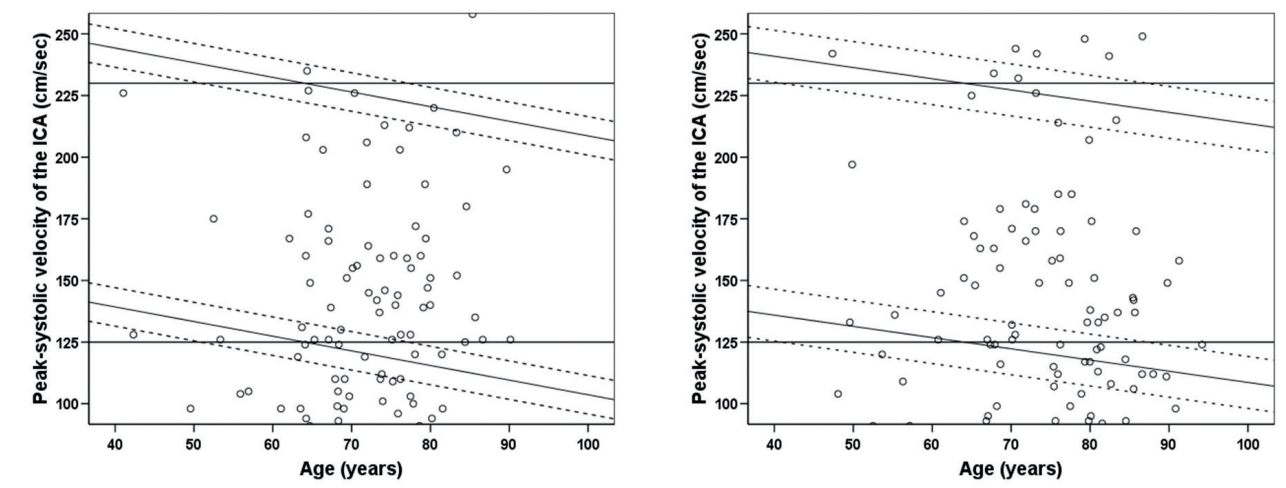

Figure 6.2. Scatter plot of peak-systolic velocity in the ICA in asymptomatic (A) and symptomatic (B) arteries with present carotid atherosclerosis. Horizontal lines at 125 and $230 \mathrm{~cm} / \mathrm{s}$ represent the references commonly used to diagnose 50\% stenosis and 70\% stenosis, respectively. The age-dependent reference lines to diagnose 50\% stenosis and 70\% stenosis with their 95\%-Cl are also shown (dashed lines). Blood flow velocities below $95 \mathrm{~cm} / \mathrm{s}$ or above $255 \mathrm{~cm} / \mathrm{s}$ are not presented. 
Ageing affects the accuracy of duplex ultrasonography in grading carotid artery stenosis

Table 6.5. Misclassification of the degree of internal carotid artery (ICA) stenosis for both symptomatic and asymptomatic arteries $(n=774)$ as established by the use of age-dependent reference lines.

\begin{tabular}{|c|c|c|c|c|}
\hline $\begin{array}{l}\text { Percentage } \\
\text { of stenosis }\end{array}$ & Method & $\begin{array}{l}\text { Asymptomatic } \\
\qquad(n=410)\end{array}$ & $\begin{array}{l}\text { Symptomatic } \\
\qquad(n=364)\end{array}$ & $\begin{array}{c}\text { Total } \\
(n=774)\end{array}$ \\
\hline \multirow[t]{2}{*}{$<50 \%$} & Absolute cut-off reference & 332 & 229 & 561 \\
\hline & Underestimated stenosis ${ }^{a}$ & $3(0-8)$ & $7(1-19)$ & $10(1-27)$ \\
\hline \multirow[t]{3}{*}{$50-69 \%$} & Absolute cut-off reference & 58 & 49 & 107 \\
\hline & Overestimated stenosis ${ }^{b}$ & $2(1-7)$ & $1(0-7)$ & $3(1-14)$ \\
\hline & Underestimated stenosis a & $0(0-3)$ & $1(0-4)$ & $1(0-7)$ \\
\hline \multirow[t]{2}{*}{$\geq 70 \%$} & Absolute cut-off reference & 20 & 86 & 106 \\
\hline & Overestimated stenosis ${ }^{b}$ & $0(0-1)$ & $0(0-3)$ & $0(0-4)$ \\
\hline
\end{tabular}

Figures in parenthesis are 95\%-Cl

a Number of arteries in which the degree of stenosis as established by the absolute reference line is underestimated when compared to the age-dependent reference line.

${ }^{b}$ Number of arteries in which the degree of stenosis as established by the absolute reference line is overestimated when compared to the age-dependent reference line. 


\section{Discussion}

Increasing age results in a linear decline in both systolic and diastolic blood flow velocities in the CCA and ICA. The observed decline is used to construct age-dependent cut-off reference lines for the classification of mild and severe internal carotid artery stenosis. Including age effects results in an increase or decrease of the estimated degree of stenosis in some subjects.

To date, several studies have demonstrated an age-dependent decline in blood flow velocities in the carotid arteries (Table 6.4). None of these studies could show such a compelling age-related decline in flow velocity as found in the present study. However, previous research included only healthy subjects (i.e. absence of atherosclerotic disease or risk factors for atherosclerosis). Furthermore, subjects in our cohort were evidently older than those in previous reports. All together, we believe that our cohort reflects a clinical population more accurately.

How does the age-related decline in blood flow velocities fit into our present knowledge of normal vascular ageing and the development of atherosclerosis? With increasing age, the composition of the vascular connective tissue is altered with an increase in rigid collagen as well as a decrease in elastin. ${ }^{18}$ As a result, arteries tend to stiffen, leading to decreased distensibility. ${ }^{13,} 14$ Subsequently, to maintain compliance, the vessel responds with an enlarged vessel diameter to restore storage capacity and thus normal blood flow pattern, but with lower blood flow velocities. ${ }^{17}, 20$ An increased vessel diameter and reduced blood flow velocity cause a lower wall shear stress, which is accompanied with an adaptive enlargement of the vessel intima-media thickness. ${ }^{16,19}$ The increase in diameter fails to fully restore optimal wall shear stress, affecting endothelial function negatively. ${ }^{19,27}$ Dysfunction of endothelium is characterized by a decrease in nitric oxide release, causing diminished vasodilatation and increasing endothelial permeability, leukocyte adhesion and platelet aggregation. ${ }^{28-30}$ In addition, endothelial dysfunction is thought to precede the development of atherosclerosis through an enhancement in oxidative stress. Oxidative stress is important in the oxidation of low-density lipoprotein cholesterol by macrophages. ${ }^{31}$ Therefore, atherosclerosis is considered to be accelerated by vascular ageing. ${ }^{17}, 32$ The increase in vessel diameter and diminished vasodilatation through endothelial dysfunction are likely to cause the age-related decline in blood flow velocities, combining the effect of both structural and functional changes of ageing vessels. ${ }^{20}$

The second part of this study determined the effect of the age-dependent decline in blood flow velocity on the grading of an ICA stenosis. In 774 arteries with visible carotid artery disease, including age-induced decreased blood flow velocities led to the increase of the estimated degree of stenosis for 10 arteries at the $50 \%$ cut-off reference and for 1 artery at the $70 \%$ cut-off reference. In addition, the estimated degree of stenosis was reduced in 3 arteries. 
But to what extent do these numbers matter clinically? One might argue that the number of subjects with an underestimated degree of stenosis is rather small and misclassifications mainly pertain to missed $50-69 \%$ stenoses rather than high-grade stenoses ( $\geq 70 \%$ ). However, Rothwell et al. demonstrated that carotid surgery is of considerable benefit (number needed to treat $=9$ ) in preventing ischaemic strokes in men who have symptomatic $50-69 \%$ stenosis., 33 In our population, 8 out of 11 subjects with a possibly underrated degree of stenosis were symptomatic (all male), and therefore eligible candidates for carotid endarterectomy.1, 2 Thus, underestimation of the degree of stenosis at the 50-69\% cut-off level is clinically relevant in terms of carotid endarterectomy decision-making. To date, no evidence can sufficiently demonstrate that subjects with asymptomatic carotid stenosis of 50-69\% will benefit from carotid surgery. ${ }^{4}$

Subjects with increased estimates of the degree of stenosis may be regarded to be too old to undergo carotid endarterectomy. However, the benefit from carotid surgery tends to increase with age, as was illustrated by subgroup analysis of the pooled NASCET and ECST data. ${ }^{33}$ In addition, a recent review of the literature demonstrated that carotid endarterectomy has a combined post-operative stroke/death rate of $3.51 \%$ in subjects older than 80 years, which is comparable to the risks in younger subjects. ${ }^{34}$ Moreover, long-term relative survival in octogenarians following carotid surgery has been shown to be better than in age-matched subjects. ${ }^{35}$ Thus, selection for carotid surgery should depend on functional status and overall health rather than the age of a patient.

Age influences blood flow velocities to an extent that the diagnostic accuracy of duplex ultrasonography might be affected. Hence, one may argue that age-dependent duplex criteria to diagnose carotid artery stenosis are required. This may involve a lot of effort, since conventional angiography is rarely performed on a large scale nowadays. ${ }^{6}$ A second straightforward solution is to employ the absolute cut-off guidelines less rigidly in patients of advanced age. Blood flow velocities approximating the currently used threshold values might be considered as stenosis of 50 or $70 \%$. However, the best approach might be to perform a repeated blinded carotid duplex by a second sonographer. Consistent borderline values should be regarded to account for age-related misclassification. Discrepancies between the 2 measurements should be complemented by other non-invasive techniques (e.g. magnetic resonance angiography, MRA, or computer tomographic angiography, CTA), ${ }^{7,12}$

The present study has certain limitations. First, the degree of carotid artery stenosis was not established by angiography or other imaging modalities (e.g. MRA or CTA). We believe that further research, employing MRA or CTA as a reference, is needed to corroborate that the outcome of duplex ultrasonography is indeed affected by increasing age. ${ }^{12}$ Secondly, the age-dependent reference lines were derived from a subpopulation without atherosclerosis, which was significantly younger (59.0 \pm 14.6 years) than the population with atherosclerosis (70.6 \pm 10.5 years). Exchanging the correlation between age and blood flow velocity between the subgroups with different ages might result in 
an incorrect estimate of the ageing effect. However, Figure 6.1 indicates that the relation between age and blood flow velocity is linear. Hence, we have extrapolated the correlation between age and blood flow velocity from the unaffected group to the subgroup with atherosclerosis. This extrapolation is an approximation of true relation between blood flow velocity and age in the atherosclerosis subjects, which is not corrected for possible confounders. To what level the extrapolation is justified remains unclear, since there are no studies available describing the effects of ageing on blood flow velocities in both nonatherosclerotic and atherosclerotic subgroups of similar age. 


\section{Conclusion}

To conclude, our results indicate that blood flow velocities throughout the carotid artery system decrease continuously with increasing age. This may lead to misclassification of the degree of internal carotid artery stenosis in young subjects as well as in patients of advanced age. A second blinded duplex study or the use of supplementary noninvasive imaging modalities in doubtful cases are sensible. Misclassification of the degree of stenosis has major consequences for the therapeutic strategy to prevent secondary ischaemic stroke.

\section{Acknowledgments}

This study was supported by Senter (Ministry of Economic Affairs), grant IS42014. 


\section{References}

1. Rothwell PM, Eliasziw M, Gutnikov SA, Fox AJ, Taylor DW, Mayberg MR, et al. Analysis of pooled data from the randomised controlled trials of endarterectomy for symptomatic carotid stenosis. Lancet. 2003;361:107-116

2. Bond R, Rerkasem K, Cuffe R, Rothwell PM. A systematic review of the associations between age and sex and the operative risks of carotid endarterectomy. Cerebrovascular diseases. 2005;20:69-77

3. European Stroke Organisation Executive Committee ESOW. Guidelines for management of ischaemic stroke and transient ischaemic attack 2008. Cerebrovascular diseases. 2008;25:457-507

4. Chambers BR, Donnan GA. Carotid endarterectomy for asymptomatic carotid stenosis. The Cochrane database of systematic reviews. 2005:CD001923

5. Cardona P, Rubio F, Martinez-Yelamos S, Krupinski J. Endarterectomy, best medical treatment or both for stroke prevention in patients with asymptomatic carotid artery stenosis. Cerebrovascular diseases. 2007;24 Suppl 1:126-133

6. Dinkel HP, Moll R, Debus S. Colour flow doppler ultrasound of the carotid bifurcation: Can it replace routine angiography before carotid endarterectomy? British Journal of Radiology. 2001;74:590-594

7. Schellinger PD, Richter G, Kohrmann M, Dorfler A. Noninvasive angiography (magnetic resonance and computed tomography) in the diagnosis of ischemic cerebrovascular disease. Techniques and clinical applications. Cerebrovascular diseases. 2007;24 Suppl 1:16-23

8. Wells PN. Doppler studies of the vascular system. European Journal of Ultrasound. 1998;7:3-8

9. Horrow MM, Stassi J, Shurman A, Brody JD, Kirby CL, Rosenberg HK. The limitations of carotid sonography: Interpretive and technology-related errors. American journal of roentgenology. 2000;174:189-194

10. Jahromi AS, Cina CS, Liu Y, Clase CM. Sensitivity and specificity of color duplex ultrasound measurement in the estimation of internal carotid artery stenosis: A systematic review and metaanalysis. Journal of vascular surgery. 2005;41:962-972

11. Grant EG, Benson CB, Moneta GL, Alexandrov AV, Baker JD, Bluth El, et al. Carotid artery stenosis: Gray-scale and doppler us diagnosis--society of radiologists in ultrasound consensus conference. Radiology. 2003;229:340-346

12. Wardlaw JM, Chappell FM, Best JJ, Wartolowska K, Berry E, Research NHS, et al. Non-invasive imaging compared with intra-arterial angiography in the diagnosis of symptomatic carotid stenosis: A meta-analysis. Lancet. 2006;367:1503-1512

13. Reneman RS, van Merode T, Hick P, Muytjens AM, Hoeks AP. Age-related changes in carotid artery wall properties in men. Ultrasound in medicine \& biology. 1986;12:465-471

14. Hansen F, Mangell P, Sonesson B, Lanne T. Diameter and compliance in the human common carotid artery--variations with age and sex. Ultrasound in medicine \& biology. 1995;21:1-9

15. Benetos A, Laurent S, Hoeks AP, Boutouyrie PH, Safar ME. Arterial alterations with aging and high blood pressure. A noninvasive study of carotid and femoral arteries. Arteriosclerosis and Thrombosis. 1993;13:90-97

16. Bots ML, Hofman A, Grobbee DE. Increased common carotid intima-media thickness. Adaptive response or a reflection of atherosclerosis? Findings from the Rotterdam study. Stroke. 1997;28:2442-2447

17. Kiechl S, Willeit J. The natural course of atherosclerosis. Part II: Vascular remodeling. Bruneck study group. Arteriosclerosis, thrombosis, and vascular biology. 1999;19:1491-1498 
18. Najjar SS, Scuteri A, Lakatta EG. Arterial aging: Is it an immutable cardiovascular risk factor? Hypertension. 2005;46:454-462

19. Samijo SK, Willigers JM, Barkhuysen R, Kitslaar PJ, Reneman RS, Brands PJ, et al. Wall shear stress in the human common carotid artery as function of age and gender. Cardiovascular Research. 1998;39:515-522

20. Schmidt-Trucksass A, Grathwohl D, Schmid A, Boragk R, Upmeier C, Keul J, et al. Structural, functional, and hemodynamic changes of the common carotid artery with age in male subjects. Arteriosclerosis, thrombosis, and vascular biology. 1999;19:1091-1097

21. Muller M, Schimrigk K. A comparative assessment of cerebral haemodynamics in the basilar artery and carotid territory by transcranial doppler sonography in normal subjects. Ultrasound in medicine \& biology. 1994;20:677-687

22. Scheel P, Ruge C, Schoning M. Flow velocity and flow volume measurements in the extracranial carotid and vertebral arteries in healthy adults: Reference data and the effects of age. Ultrasound in medicine \& biology. 2000;26:1261-1266

23. Yazici B, Erdogmus B, Tugay A. Cerebral blood flow measurements of the extracranial carotid and vertebral arteries with doppler ultrasonography in healthy adults. Diagnostic and Interventional Radiology. 2005;11:195-198

24. Kalvach P, Gregova D, Skoda O, Peisker T, Tumova R, Termerova J, et al. Cerebral blood supply with aging: Normal, stenotic and recanalized. Journal of the neurological sciences. 2007;257:143-148

25. Touboul PJ, Hennerici MG, Meairs S, Adams H, Amarenco P, Bornstein N, et al. Mannheim carotid intima-media thickness consensus (2004-2006). An update on behalf of the advisory board of the 3rd and 4th watching the risk symposium, 13th and 15th European stroke conferences, Mannheim, Germany, 2004, and Brussels, Belgium, 2006. Cerebrovascular diseases. 2007;23:75-80

26. Henderson RD, Steinman DA, Eliasziw M, Barnett HJ. Effect of contralateral carotid artery stenosis on carotid ultrasound velocity measurements. Stroke. 2000;31:2636-2640

27. Reneman RS, Arts T, Hoeks AP. Wall shear stress--an important determinant of endothelial cell function and structure--in the arterial system in vivo. Discrepancies with theory. Journal of Vascular Research. 2006;43:251-269

28. Davignon J, Ganz P. Role of endothelial dysfunction in atherosclerosis. Circulation. 2004;109:III27-32

29. Landmesser $\mathrm{U}$, Drexler $\mathrm{H}$. The clinical significance of endothelial dysfunction. Current opinion in cardiology. 2005;20:547-551

30. Zalba G, Fortuno A, San Jose G, Moreno MU, Beloqui O, Diez J. Oxidative stress, endothelial dysfunction and cerebrovascular disease. Cerebrovascular diseases. 2007;24 Suppl 1:24-29

31. Ross R. Atherosclerosis--an inflammatory disease. The New England journal of medicine. 1999;340:115-126

32. Lakatta EG, Levy D. Arterial and cardiac aging: Major shareholders in cardiovascular disease enterprises: Part I: Aging arteries: A "set up" for vascular disease. Circulation. 2003;107:139-146

33. Rothwell PM, Eliasziw M, Gutnikov SA, Warlow CP, Barnett HJ, Carotid Endarterectomy Trialists C. Endarterectomy for symptomatic carotid stenosis in relation to clinical subgroups and timing of surgery. Lancet. 2004;363:915-924

34. Miller MT, Comerota AJ, Tzilinis A, Daoud Y, Hammerling J. Carotid endarterectomy in octogenarians: Does increased age indicate "high risk?". Journal of vascular surgery. 2005;41:231-237

35. Norman PE, Semmens JB, Laurvick CL, Lawrence-Brown M. Long-term relative survival in elderly patients after carotid endarterectomy: A population-based study. Stroke. 2003;34:e95-98 



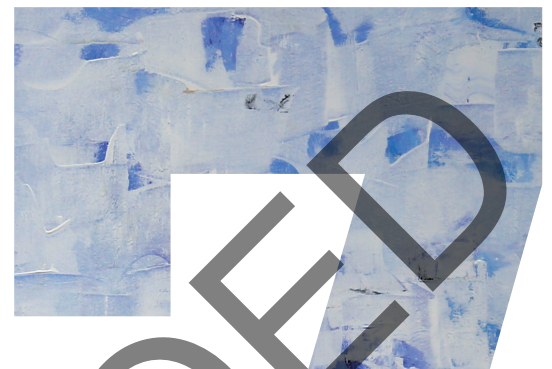

\section{SIMULTANEOUS INVESTIGATION OF STRUCTURAEAND FUNCTIONAL PARAMETERS OF CAROTID ARTERY PLAQUE USING ROUTINE B-MODE} ULTRASONOGRAPHY: THE PLAQUE AT RISK (PARISK) STUDY

FHBM Schreuder, J Steinbuch, APG Hoeks, JC Sluimer, RJ van Oostenbrugge, ME Kooi, WH Mess

Submitted 


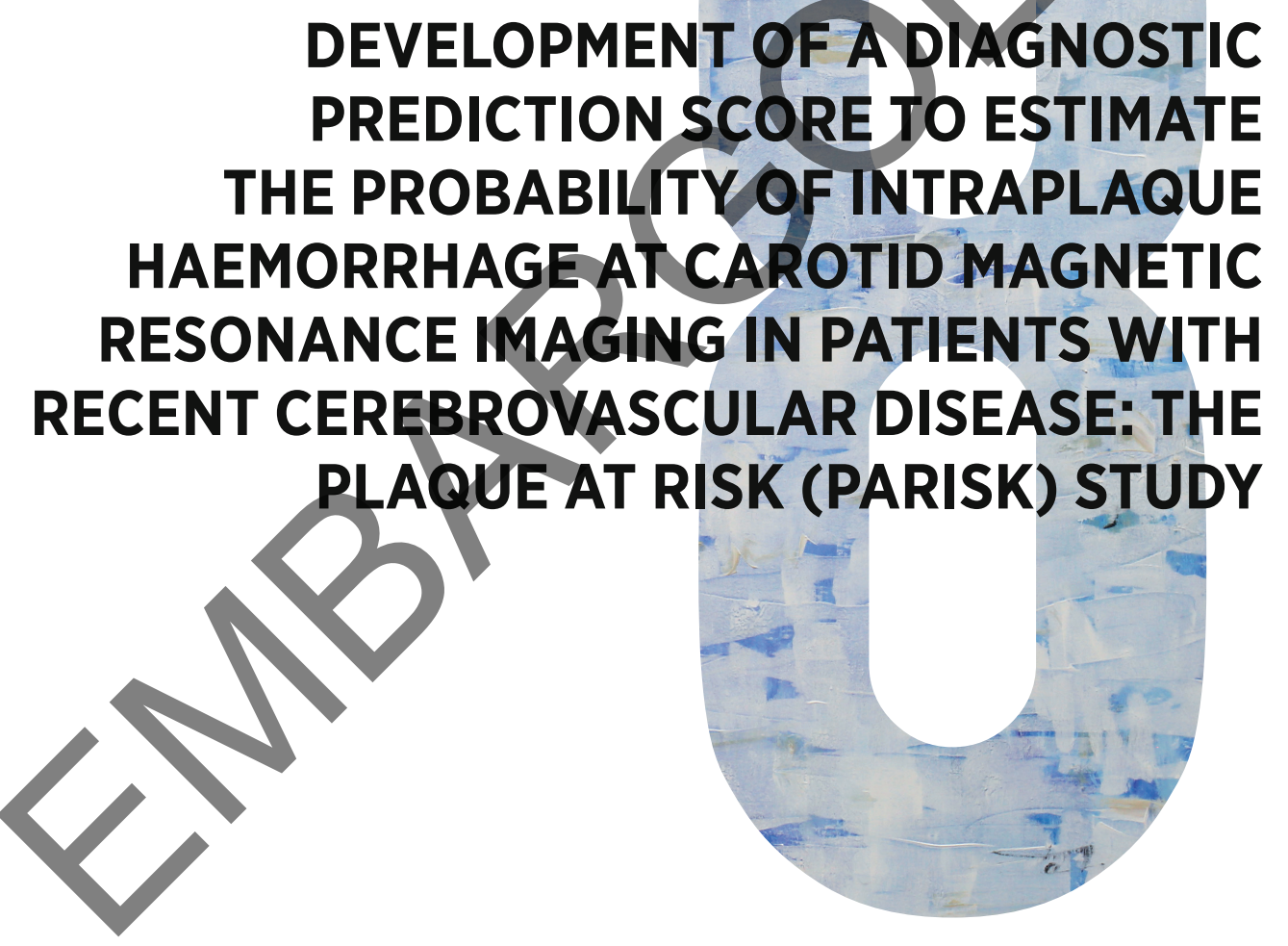

FHBM Schreuder, J Steinbuch, RHM van Hoof, NA Hilkens, HK van Dam-Nolen, AG van der Kolk, TJ van Velzen, MJAP Daemen, J Hendrikse, PJ Nederkoorn, A van der Lugt, RJ van Oostenbrugge, WH Mess, ME Kooi 


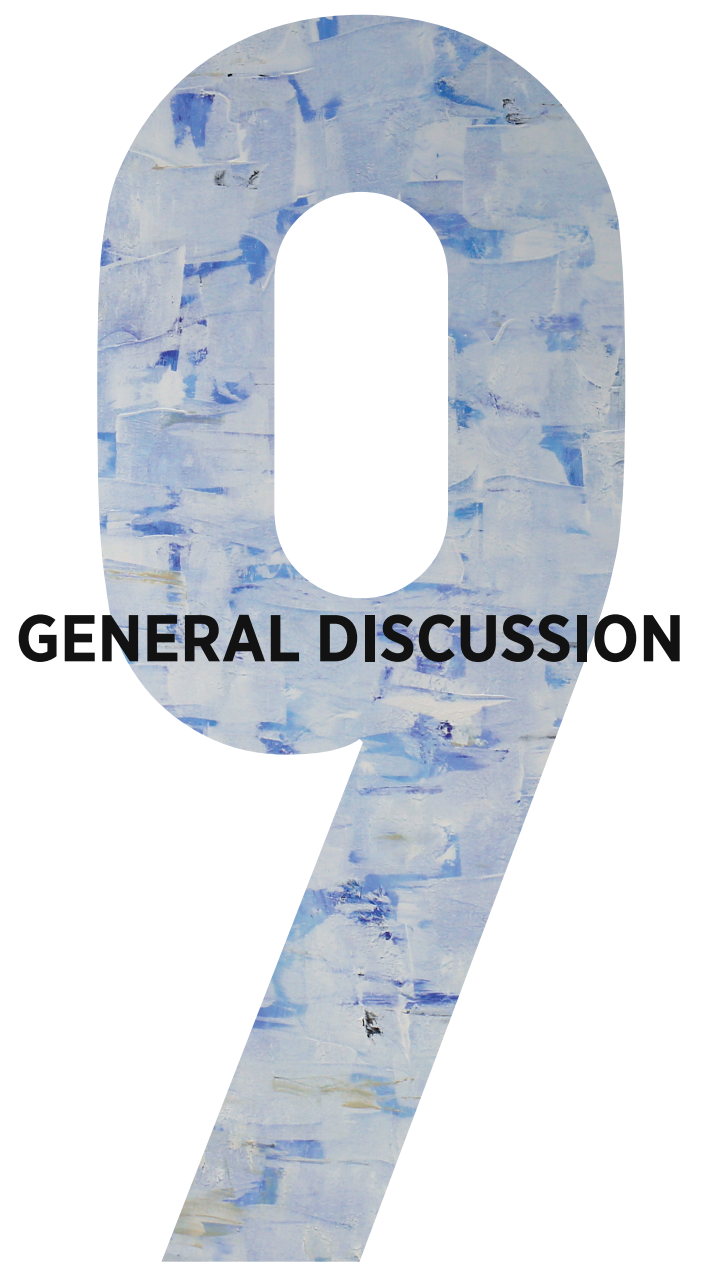


Trying to treat arteries without measuring plaque is like treating hypertension without measuring blood pressure. 


\section{Introduction}

Ischemic stroke is a major burden on health care around the world. In 2016, an estimated 13.7 million people had an ischemic stroke of whom 5.5 million died. ${ }^{2}$ Despite vast improvements in the understanding of stroke pathophysiology and major progress in imaging techniques and acute stroke treatments, the impact of stroke on global health care is still overwhelming. On the level of an individual patient, the impact of having a stroke is even more devastating. Stroke often results in physical deficits, psychological symptoms, functional dependency and societal sequalae, which taken together can have a large effect on the quality of life after stroke. ${ }^{3}$

In $15-20 \%$ of patients with ischemic stroke, an atherosclerotic plaque is present within the carotid bifurcation or internal carotid artery causing a moderate to severe degree of luminal stenosis ( $\geq 50 \%$ ). In such patients, stroke is thought to result from rupture of the luminal plaque surface, with subsequent shedding of thromboemboli into the intracranial vasculature. In order to prevent stroke recurrence, current guidelines advocate the surgical removal of moderate to severe carotid stenosis by means of carotid endarterectomy (CEA), preferably within 2 weeks after symptom onset. ${ }^{4}$ Yet, in recently symptomatic patients with $70-99 \%$ stenosis, the number needed to treat to prevent one ipsilateral stroke in the next five years is six. In patients with 50-69\% stenosis, the number needed to treat increases to $22 .{ }^{5} \mathrm{It}$ is increasingly recognized that the structural composition of the plaque is an important predictor of future ischemic events, irrespective of the degree of stenosis. Intraplaque hemorrhage (IPH) is a hallmark of plaque vulnerability, and it can be reliably imaged in vivo using carotid MRI. In a recent individual patient data metaanalysis including 560 patients with prior ischemic stroke or TIA, the presence of IPH was strongly associated with an increased risk of ipsilateral stroke recurrence during 16.7 months of follow-up (hazard ratio 10.2, 95\%-Cl 4.6-22.5). Moreover, in 136 patients with an asymptomatic carotid artery stenosis with a mean follow-up duration of 30.1 months, the hazard of ischemic stroke was similarly increased (hazard ratio 7.9, 95\%-Cl 1.3-47.6). ${ }^{6}$

In clinical practice, risk stratification still almost exclusively depends on the degree of luminal stenosis to select recently symptomatic patients for CEA. This has several important pitfalls. First, purely selecting patients based on the degree of stenosis will result in treatment of patients who will not benefit from CEA while unnecessarily exposing them to the risks of surgery. Second, non-stenotic ( $<50 \%$ diameter reduction) carotid plaques are present in up to $40 \%$ of patients in whom no cause for stroke can be established despite extensive work-up., ${ }^{7,8}$ Up to a third of patients with $<50 \%$ stenosis and an embolic stroke of undetermined source harbor plaques with structural features associated with a high risk of stroke recurrence. ${ }^{9}$ Third, atherosclerotic plaques slowly develop over the course of many years before they become symptomatic. Consequently, asymptomatic moderate ( $\geq 50 \%$ stenosis) carotid artery plaques can be found in $4 \%$ of the population, whereas asymptomatic severe $(\geq 70 \%)$ stenosis are present of $1.7 \%$ of people. ${ }^{10}$ Current 
guidelines contradict on the optimal management for people with asymptomatic carotid artery stenosis." As a result, the proportion of patients with asymptomatic carotid artery disease undergoing carotid revascularization largely differs between countries. In 2018, more than 2,500 patients underwent carotid revascularization in the Netherlands.12 Of these, $97 \%$ had prior symptoms of an ischemic stroke or transient ischemic attack. In Germany however, only $34 \%$ of annual 42,000 carotid revascularization procedures are performed in symptomatic patients. ${ }^{13}$

Taken together, current risk stratification requires modifications to better select patients for carotid revascularization. Improving our understanding in the process of plaque destabilization ultimately resulting in plaque rupture will help to improve patient selection. Besides plaque structural composition, biomechanical properties eventually triggering plaque rupture should also be considered. Carotid ultrasonography could be used as a non-invasive technique to simultaneously study plaque structural and functional properties in patients with recent cerebrovascular disease.

This thesis aims at contributing to the development and validation of ultrasonography (US) techniques which enable in vivo assessment of structural and functional vessel wall and plaque properties, as to gain more insight in the pathophysiology of plaque development eventually resulting in plaque rupture. More specifically, this thesis addresses the following objectives:

- To evaluate different ultrasonography techniques investigating the structural and functional properties of common carotid artery (CCA) vessel wall and atherosclerotic plaque in the CCA;

- To gain insight in the interplay between structural and functional properties of CCA vessel wall and more distally located carotid plaque at the carotid bifurcation;

- To use these ultrasonography techniques in the anatomically more complex carotid artery bifurcation to study the simultaneous assessment of structural and functional plaque properties.

The general discussion of this thesis will address my results in relation to the hypothesis that the interplay between plaque structural composition and plaque biomechanical properties ultimately results in plaque destabilization and plaque rupture. Furthermore, I will discuss methodological and technical considerations, clinical implications and future perspectives. 


\section{The role of biomechanical forces in plaque destabilization}

Plaque destabilization is a dynamic process which is only partly understood. As introduced in Chapter 1, key pathophysiological factors that presumably contribute to plaque rupture are plaque composition, plaque inflammation and biomechanical forces working on the plaque. These features are strongly interrelated, but biomechanical stress can trigger several events implicated in the development of vulnerable plaques. However, as most research in the field of plaque destabilization is cross-sectional, it remains difficult to unravel the sequence of events leading to plaque rupture. Grossly two biomechanical forces affecting the plaque luminal surface may contribute to the process of destabilization: wall shear stress and cyclic tensile stress (Figure 9.1).

First, wall shear stress (WSS) is the tangential drag force exerted by the flowing blood over the plaque surface. It can be estimated as the product of blood viscosity and wall shear rate, the latter being defined as the radial derivative of blood flow velocity at the luminal surface. ${ }^{14}$ WSS is known to play an important role in endothelial dysfunction leading to early plaque formation in regions with low WSS. ${ }^{15}$ WSS however is also thought to affect plaque stability. As blood flow velocity exponentially rises at the level of the plaque with increasing degree of stenosis, WSS will also rise at the proximal plaque shoulder (i.e. the upstream part of the plaque). ${ }^{16,17}$ Prolonged elevated WSS induces apoptosis of vascular smooth muscle cells and stimulates expression of endothelial leukocyte adhesive molecules resulting in an influx of monocytes. ${ }^{18,19}$ Monocytes oxidate LDL-cholesterol to form foam cells, that die from apoptosis and necrosis to enhance growth of the lipid-rich, necrotic core (LRNC). Moreover, these inflammatory cells produce cytokines, proteolytic enzymes and other molecules that degrade the extracellular matrix, thus weakening the overlying fibrous cap. To summarize, high WSS is thought to induce the transformation of the plaque to a more rupture-prone state and promotes plaque growth, with subsequent further increase in WSS. Estimation of WSS is feasible with dedicated US techniques ${ }^{15,20}$, whereas MRI-based modelling has also been applied to estimate WSS. Plaque inflammation can also be quantified in vivo by means of Positron Emission Tomography (PET) imaging, which correlates with symptomatic plaque status. ${ }^{21,22}$ WSS and plaque inflammation are however beyond the scope of this thesis.

Second, cyclic tensile stress results from the pulsatile blood flow within arteries. The pulse pressure, i.e. the difference between systolic and diastolic blood pressure, exerts biomechanical load to the proximal plaque shoulder. Because of luminal narrowing caused by the plaque, local pulse pressure at the proximal plaque shoulder is even increased because of reflection of the arterial pressure wave. This results in a longitudinal pressure gradient across the plaque. Additionally, radial pressure also increases with every heartbeat, distending the artery diameter and compressing the plaque. As a result of the repetitive pressure gradient the plaque will deform to more or lesser extent during the 

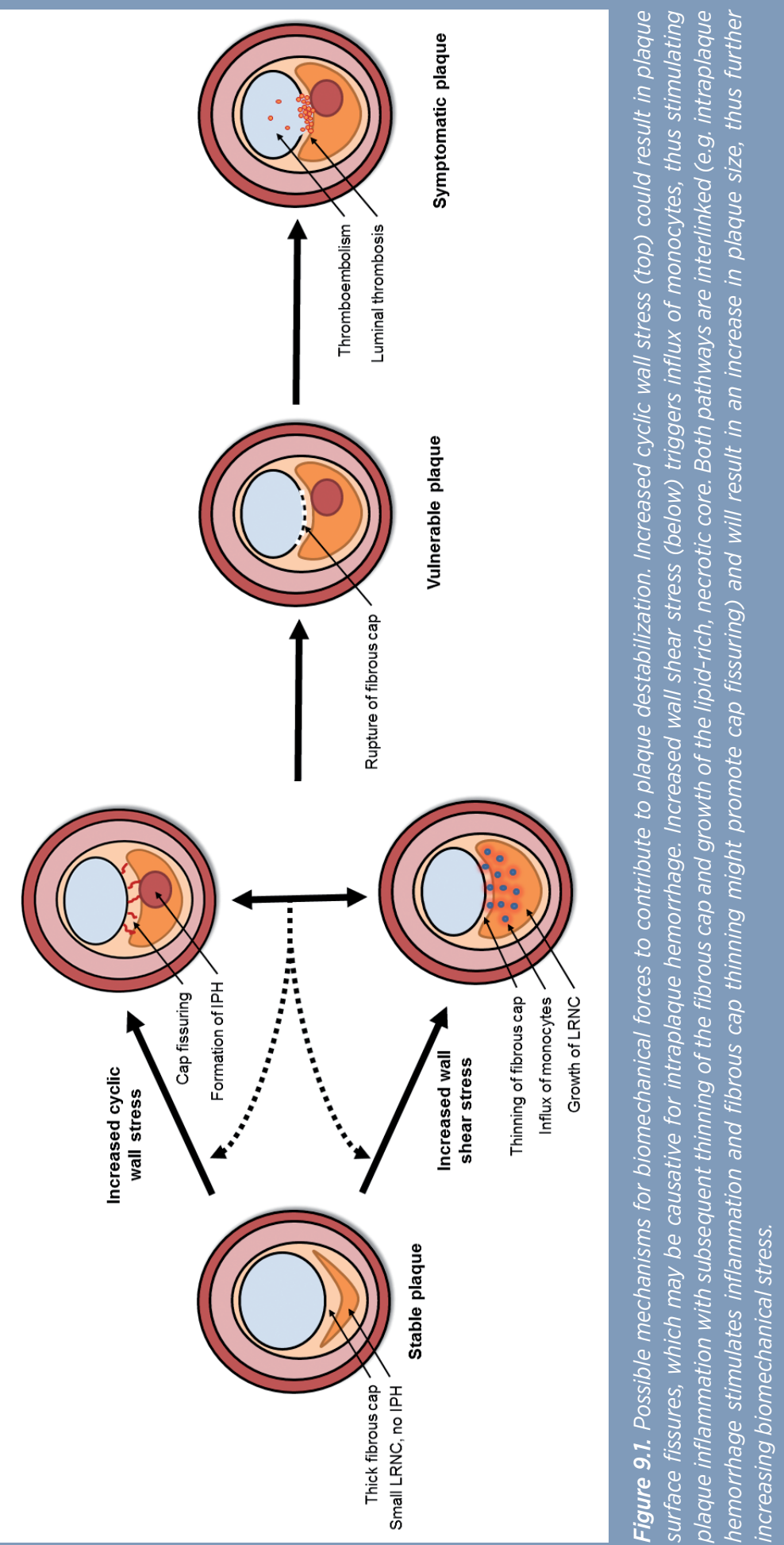
cardiac cycle, resulting in intraplaque strain (Figure 1.7). Repetitive plaque deformation might ultimately lead to plaque rupture due to material fatigue when ultimately the biomechanical stress exceeds the strength of the plaque. ${ }^{23,24}$ It should be noted that local pulse pressure is more likely to trigger plaque rupture than WSS, as the pressure gradient can be a factor 100 larger than WSS. ${ }^{25,26}$

Besides playing an essential role in eventual plaque rupture, cyclic wall stress may also contribute to plaque destabilization through fresh IPH or the expansion of existing $\mathrm{IPH}$. IPH is associated with rapid increase in plaque volume. ${ }^{27}$ Also, IPH induces a strong inflammatory response due to the degradation of erythrocytes. Repetitive plaque deformation may result in small fissures in the fibrous cap overlying the plaque. Fissures were associated with fresh IPH in $63 \%$ and with any IPH in $92 \%$ of cases, in a study including 63 carotid specimens. ${ }^{28}$ Moreover, $43 \%$ of fissures were localized near small juxtaluminal calcifications, which can exaggerate local biomechanical stress. Fissures and ulcerations most often occur at the upstream plaque shoulder in histopathological ${ }^{28-30}$ and imaging studies $^{31}$. Using data from the PARISK study, we recently confirmed that IPH and thin/ ruptured fibrous cap were more prevalent in the proximal plaque shoulder in 88 patients with mild to moderate carotid stenosis with IPH, supporting a role for biomechanics and hemodynamics in the formation of IPH. ${ }^{32}$ Interestingly, pulse pressure was the strongest determinant of IPH in 1,006 participants of the Rotterdam study, independent of other blood pressure components and cardiovascular risk factors. ${ }^{33}$ In a meta-analysis of 11 studies including 129,901 subjects, a $10 \mathrm{mmHg}$ increase in pulse pressure was associated with an increased risk of stroke (pooled hazard ratio 1.05; 95\%-Cl 1.03-1.07). ${ }^{34}$

Taken together, these observations suggest that plaque biomechanics play a pivotal role in plaque destabilization and rupture. Plaques that are characterized by increased deformation are likely more susceptible to rupture. Moreover, structural properties and biomechanical behavior of non-stenosed vessel walls and of plaques are likely to interact, as was shown in this thesis.

\section{Structural vessel wall parameters}

In the first part of this thesis, I used the common carotid artery (CCA) as a substitute to the more complex carotid artery bifurcation and internal carotid artery (ICA). Moreover, I first studied the arterial vessel wall before proceeding towards plaque imaging.

Because thickening of the arterial wall must precede the development of plaque, US measurement of the thickness of the "normal", non-stenosed vessel wall at the level of the CCA gained interest in the late 1980s. Thickness of the intima-media segment at the posterior vessel wall of the CCA (CCA-IMT) was considered to reflect preclinical atherosclerotic disease and, as such, thought to be a valuable biomarker or even a surrogate endpoint for randomized trials aimed at reducing atherosclerotic disease 
burden. ${ }^{35,36}$ However, IMT adds little to individual risk prediction. In an individual patient data meta-analysis including 45,828 individuals from 14 population-based studies, prediction of the 10-year risk of stroke and myocardial infarction using the Framingham Risk Score was only marginally improved by adding CCA-IMT to the prediction model (net reclassification improvement of $0.8 \%) .{ }^{37}$ Increased CCA-IMT probably reflects vascular ageing, compensating for the loss in vessel wall elasticity. ${ }^{38} \mathrm{~A}$ recent meta-analysis confirmed that the annual increase in CCA-IMT was linear with age in 32 studies involving 10,124 healthy individuals, and that in populations with cardiovascular risk factors or overt cardiovascular disease (115 studies, 65,774 individuals), the annual increase in CCA-IMT was comparable to that of asymptomatic individuals. ${ }^{39}$

Thus, alternative approaches were examined to improve our understanding of preclinical atherosclerosis. Variations in the thickness along the length of the arterial wall can be observed in some patients, and this spatial inhomogeneity of CCA-IMT was hypothesized to more truly reflect atherosclerotic disease..$^{40}$ The revised Mannheim Carotid Intima-Media Thickness and Plaque Consensus (2011) specify when a vessel wall irregularity should be regarded as a plaque. In this consensus, plaque is defined as a focal structure that encroaches into the arterial lumen of at least $0.5 \mathrm{~mm}$ or $50 \%$ of the surrounding IMT value or demonstrates a thickness $>1.5 \mathrm{~mm} .{ }^{41}$ People with irregular vessel walls might be more likely to harbor atherosclerotic vessel wall changes and thus might be more prone to develop symptoms, as compared to people with a smoothly thickened vessel wall, despite a similar mean IMT value. ${ }^{42}$ In Chapter $\mathbf{2}$ we demonstrate that spatial inhomogeneity of CCA-IMT might be a measure for a more advanced atherosclerotic disease. The main aim of this study was to compare the routine B-mode approach and the radiofrequency (RF) based technique to measure CCA-IMT in 136 patients (72\% with recent cerebrovascular disease). Although the methods correlated well and had similar reproducibility, B-mode CCA-IMT was significantly larger than the RF-based measurements. The RF-based technique allowed the measurement of spatial inhomogeneity of CCA-IMT, which was defined as the standard deviation of the spatial distribution of the CCA-IMT over the available 12 echo lines covering $13.9 \mathrm{~mm}$. Spatial inhomogeneity of the CCA-IMT was independently associated with a discrepancy between the two methods in CCA-IMT values. Other predictors of a difference between the two techniques were plaque at the carotid bifurcation and a history of diabetes mellitus, suggesting that spatial inhomogeneity of CCA-IMT could possibly reflect more advanced atherosclerosis. We have explored this observation in more detail in 147 patients using radiofrequency (RF) multiple M-line analysis with $12 \mathrm{M}$-lines covering $13.9 \mathrm{~mm}$ (Chapter 3). The spatial inhomogeneity, as well as the variation of CCA-IMT between two repeated measurements and the variation of CCA-IMT between the left and right artery were associated with the extent of more distally located atherosclerotic disease. We later confirmed the hypothesis that vessel wall irregularity was associated with more advance atherosclerotic disease. Using the baseline data from the PARISK study, 40 patients with 
a high CCA-IMT inhomogeneity ( $>2 \%$ ) more often had a larger degree of stenosis on CTangiography compared to 142 patients with low CCA-IMT $(<2 \%)$ inhomogeneity, even after adjustment for cardiovascular risk factors. ${ }^{43}$ The absolute difference in degree of stenosis was however small ( $52 \%$ vs. $47 \%, p=0.023$ ). Whether high CCA-IMT inhomogeneity can be used to predict future cerebrovascular events will be studied in the longitudinal follow-up phase of the PARISK study.

\section{Functional vessel wall parameters}

Even before resulting in visible structural vessel wall changes (i.e. wall irregularities or plaques), atherosclerosis affects the vascular wall. In healthy arteries, the elastic vessel wall properties allow the arterial diameter to change when local pulse pressure is increased or decreased. The diastolic-to-systolic variation of diameter is referred to as distension. ${ }^{44}$ Both "normal" vascular ageing and atherosclerosis alter vessel wall elastic properties, resulting in reduced distension of otherwise normal-appearing CCA vessel wall. The process of arterial stiffening can be quantified using the carotid-femoral pulse wave velocity (PWV), averaging the propagation speed of the arterial waveform over both the elastic (aorta, carotid) and muscular (femoral) components of the vascular tree. Carotid-femoral PWV has been associated with cardiovascular events and allcause mortality. ${ }^{45}$ However, arterial stiffening is not uniformly distributed and may affect elastic and muscular arteries differently. Additionally, carotid-femoral PWV velocity disregards local variations in arterial stiffness, which may be more strongly associated with atherosclerosis than with vascular ageing. A meta-analysis including 22,472 patients from ten studies established that local carotid stiffness is associated with stroke (hazard ratio 1.18; 95\%-Cl 1.05-1.33), total cardiovascular events (hazard ratio 1.16; 95\%-Cl 1.071.26) and cardiovascular mortality (hazard ratio 1.30; 95\%-Cl 1.15-1.46). Including carotidfemoral PWV measurement did not alter these associations. ${ }^{46}$

US can be used to study local arterial distension and can incorporate inhomogeneity of vessel wall distension as possible parameters of local atherosclerotic changes. In Chapter $\mathbf{4}$ we have shown that plaque at the carotid bifurcation was weakly associated with reduced local absolute and relative distension in 154 patients. A large intermeasurement variation in local absolute and relative distension was however strongly associated with distally located stenosis, independent of age, gender and pulse pressure. Similar to Chapter 3 , the inter-measurement variation of distension can be regarded as a measure of local distension inhomogeneities, because repositioning of the US probe will measure the arterial structural or functional properties at a slightly different position. This indicates that distension inhomogeneity is a marker of atherosclerotic changes within the vessel wall. We have recently confirmed these results using an alternative measure for spatial distension inhomogeneity. Using routine B-mode US acquired at the baseline visit from 211 patients in the PARISK study, we divided the CCA in 15 half-overlapping 
segments (with a width of $3.7 \mathrm{~mm}$ ) to derive multiple distension curves along an arterial segment. We studied the spatial inhomogeneity of the diastolic-to-systolic distension risetime and correlated it with plaque composition at the carotid bifurcation on MRI. We found that spatial inhomogeneities in the CCA risetime were associated with vulnerable plaques features, such as a thin/ruptured fibrous cap or a large LRNC within the proximal plaque shoulder. ${ }^{47}$ We interpreted these findings as a cushioning effect of vulnerable plaques on arterial wave reflections, which results from a decrease of fibrous tissue content or an increase in soft lipid content, thus altering the ability to reflect pressure waves. These results indicate that the functional vessel wall properties are associated with the composition of more distally located plaques.

\section{Structural plaque parameters}

In this discussion, we move from subclinical atherosclerotic vessel wall alterations to structural plaque properties. There are two key aspects of carotid plaque morphology that can be studied with US in an attempt to improve individual risk stratification. First, plaque dimensions can be extracted to determine the atherosclerotic burden. Total plaque area and maximal plaque thickness are the most important plaque dimensions and have been associated with ipsilateral ischemic stroke recurrence in symptomatic patients and cardiovascular events in asymptomatic subjects. ${ }^{48-50}$ Second, the amount of reflected ultrasound from the plaque can be analyzed to evaluate plaque composition. Plaque echogenicity is a well-known measure of plaque composition and has shown its predictive value to identify high-risk plaques..$^{51-54}$ Grey-scale median (GSM) averages echogenicity of all pixels within a designated plaque area, usually on a 8-bit range with 0 being totally echolucent and 255 indicating the maximal echointensity. In Chapter 7 we confirm that a low GSM is associated with the presence of a lipid-rich necrotic core and/or intraplaque haemorrhage in histological specimens of 18 patients who underwent carotid endarterectomy (CEA). This is in line with earlier observations. ${ }^{52,53,55}$ Some authors reported a high GSM being indicative of calcifications ${ }^{55}$, 56, where others, like we did, found no such association..$^{53}$ Averaging echogenicity over the entire plaque may discard important local variation in echogenicity, which represents local inhomogeneities in plaque composition possibly contributing to plaque destabilization. More detailed analyses of plaque texture are available, including spatial variations of GSM in relation to the plaque luminal surface, but are beyond the scope of this thesis. ${ }^{55,57}$

I next focus towards measurement of stenosis at the carotid artery bifurcation. To date, clinical duplex US depends on Doppler measurement of local blood flow velocities to determine the degree of stenosis. Guidelines define stringent absolute cut-off criteria to discriminate between $<50 \%$ stenosis and $50-69 \%$ stenosis (peak-systolic velocity [PSV] at the ICA of $125-230 \mathrm{~cm} / \mathrm{s}$ signifies a stenosis of $50-69 \%$ ) and to diagnose $\geq 70 \%$ stenosis (PSV at the ICA $>230 \mathrm{~cm} / \mathrm{s}$ ) ${ }^{58}$ Blood flow velocity is however inversely associated with 
increasing age, because of an increase in rigid collagen and a loss of elastin within vessel walls. As a result, arterial walls will be stiffer, and to maintain compliance, arteries will increase diameter to improve storage capacity at the cost of lower blood flow velocities. In Chapter 6, we have used retrospective data from 810 patients who underwent duplex US of the carotid arteries to confirm that PSV and end-diastolic velocity (EDV) linearly decrease with age in both the CCA and ICA. As a result, handling strict cut-off values of blood flow velocities might result in misclassification of the degree of stenosis in both the young (an overestimation of stenosis severity) and the old (an underestimation of stenosis severity). The total number of misclassifications was small (14 of 774 arteries) and these data need to be reproduced in a prospective cohort with CTA or MRA to establish the degree of stenosis. Baseline data from the two sub-studies within the PARISK project might be used for such a purpose.

\section{Functional plaque parameters}

The CCA is next used to examine functional plaque properties with US. Compared to the carotid bifurcation, the CCA has the simple configuration of a straight vessel, running parallel to the skin surface and being located at a rather superficial depth (usually 2-3 $\mathrm{cm}$ below the skin surface). However, one must realize that CCA plaques rarely lead to hemodynamical stenosis or result in clinical symptoms. ${ }^{59,60}$ In general, CCA plaques are likely to reflect the presence of more severe stages of atherosclerosis. CCA plaques are usually aligned parallel to the US transducer surface, whereas plaques in the carotid bifurcation may be at an angle of up to 45 degrees relative to the transducer surface. Such large angles complicate valid measurement of plaque parameters due to loss of depth resolution, which is most optimal if the structure under study runs perpendicular to the US transducer surface. In Chapter $\mathbf{5}$ we developed a method to study functional vessel wall and plaque properties. From the PARISK baseline cohort, we selected 23 patients in whom we used routine B-mode US to image plaques located at the posterior wall of the CCA. To overcome the loss of depth resolution, we studied distension at the internal (luminal) and external (adventitial) vessel wall along the local vessel axis instead of a plane perpendicular to the transducer surface. These orthogonal distension measurements were interspaced at $1 \mathrm{~mm}$ along the vessel longitudinal axis (usually over $3 \mathrm{~cm}$ ). At the level of the maximal plaque thickness, the distension curve was used to estimate plaque compression, i.e. the change in plaque thickness from diastole to systole. Although it was possible to reliably measure diameter and distension along the entire arterial segment and at the level of maximal plaque thickness, precision and interobserver reproducibility of plaque compression was poor. The main reason for this poor precision was the small change in plaque thickness (mean change of $8 \mu \mathrm{m}$ ), which is well below the conservatively estimated system resolution in depth $(300 \mu \mathrm{m})$. A possible solution to overcome this poor precision is to consider spatial averaging of compression 
over the entire length of the plaque. When we considered lumen distension at the site of maximal plaque thickness, we noticed a negative association with plaque echogenicity. This suggests that plaques with more stable features (e.g. high calcium content and higher amount of fibrous tissue) are stiffer compared to vulnerable plaques (e.g. large LRNC).

\section{Simultaneous assessment of structural and functional plaque parameters}

To improve the precision of measurement of functional plaque properties, we studied an alternative approach, which had the advantage that it could also derive structural plaque components. Instead of measuring compression only at the level of maximal plaque thickness, we included diastolic-to-systolic changes in plaque dimensions spatially averaged over the entire plaque. We drew regions-of-interest enclosing all plaque segments, from which we could extract structural (GSM, total plaque area, plaque length, plaque thickness and maximal plaque thickness) and functional plaque properties (diastolic-to-systolic change in plaque length and plaque thickness, and diastolic-tosystolic change in plaque center of gravity). In Chapter 7, we described the reproducibility and precision of these measures in a selection of patients included in the PARISK project. In 25 patients, structural plaque features had good to excellent inter-observer and intra-observer agreement and good precision. Measurement of diastolic-to-systolic change in plaque thickness and center of gravity was moderately reproducible and less precise, whereas reproducibility of change in plaque length was poorly reproducible and inaccurate. Compared to the results reported in Chapter 5 , precision had clearly improved, which mainly results from higher mean plaque compression ( $81 \mu \mathrm{m}$ vs. $8 \mu \mathrm{m})$. Spatial averaging of variation in plaque thickness holds the advantage of including some parts where plaque compression is larger than the depth resolution, and other parts where this is smaller than the depth resolution, thus being able to measure an average estimation of plaque compression. However, this method neglects spatial inhomogeneities in plaque compression that result from disparities in plaque thickness, composition and local pulse pressure.

When we correlated the structural and functional plaque properties with histology in 18 patients who underwent CEA, soft plaques with larger combined size of LRNC and IPH had greater changes in plaque thickness from late diastole to early systole, whereas the proportion of calcifications was inversely associated with plaque compression. GSM was also inversely associated with the proportion of LRNC and IPH consistent with existing literature. ${ }^{52,53}$ We concluded that the simultaneous measurement of structural and functional plaque properties is feasible and has modest to excellent reproducibility. Of the studied parameters, GSM and diastolic-to-systolic change in plaque thickness were most promising to further explore and determine their complementary value in identifying patients at high risk for stroke recurrence. 
In Chapter 8 we have explored the potential of simultaneously assessing structural and functional plaque properties. In 259 participants included in the two sub-studies of the PARISK project, we demonstrate that clinical and US parameters can be used to predict the presence of IPH on carotid MRI with good discrimination and calibration. Both low GSM and high diastolic-to-systolic change in plaque thickness were independent predictors for $\mathrm{IPH}$. This emphasizes the importance of simultaneous investigation of structural and functional plaque properties in risk stratification. Other important predictors were advanced age, male sex, presence of hypertension and stenosis $\geq$ $50 \%$. These predictors were combined in the PARISK-US score. Before this score can be implemented in clinical practice, these results need to be externally validated in a separate cohort. One possible cohort to use to externally validate the PARISK-US score may be the Carotid Plaque Imaging in Acute Stroke (CAPIAS) study. ${ }^{61}$ This observational cohort study is quite comparable to the PARISK observational cohort study, and has prospectively enrolled 234 participants with recent anterior circulation stroke and $<70 \%$ ipsilateral carotid stenosis at three University hospitals in Germany.

\section{Technical considerations}

As opposed to other imaging techniques (i.e. CTA or MRA), US is the only modality that can directly depict and quantify plaque deformation as a measure of functional plaque properties due to its relatively high spatial and temporal resolution. For example, several MRI-based techniques have been developed to estimate carotid artery wall and plaque dynamical parameters, such as cine MRI or black blood cine MRI. Still temporal resolution $(\sim 20 \mathrm{~Hz})$ and spatial resolution $(600-800 \mu \mathrm{m})$ are inferior compared to US. ${ }^{62}$

In this thesis, I have used routine B-mode US and RF-based techniques to study vessel wall and plaque properties. Routine B-mode images undergo adjustments such as thresholding and amplitude compression to reduce noise and improve image quality. Moreover, image processing differs between various commercially available US devices, thus limiting the external generalizability. Image sampling frequency is usually set at 25$50 \mathrm{~Hz}$. Alternatively, unprocessed RF signals can be used to analyze vessel wall and plaque properties. Advantages of RF-based image analysis include: 1) linear characteristics of gain settings; 2) offline modification of RF-data without loss of raw data; 3) image sampling frequency can be increased to reach a very high temporal resolution (200$1000 \mathrm{~Hz}$ ); 4) improved depth resolution in motion analysis to allow assessment of tissue displacement. These high frame rates improve accuracy of computing dynamic vessel wall and plaque properties, such as wave reflections. However, in our studies, increasing the temporal resolution came at the cost of decreased lateral spatial resolution (along the axis of the US probe).

RF-based US with high temporal resolution has been used to estimate plaque strain as a measure of the interplay between plaque composition and hemodynamic forces 
working on the plaque. Several methods have been suggested, such as displacement vector maps ${ }^{63,64}$, spatial distension distribution ${ }^{65,66}$, elastography ${ }^{67-70}$ or axis dependent strain imaging ${ }^{71-73}$. Whereas some of these studies have focused on strain averaged over the entire plaque, similar to our method described in Chapter 7, others have been able to provide detailed maps of strain distribution throughout the plaque. Sufficient spatial resolution is the main requirement to allow for estimation of local strain inhomogeneity within the plaque. RF-based techniques with even higher temporal resolution and with preserved spatial resolution currently become available, increasing the number of frames per second up to 10,000. One of these novel techniques is velocity vector imaging, which can be used to study the multidirectional flow patterns at the level of the plaque surface to estimate local WSS. ${ }^{20,74}$ Investigating of local strain inhomogeneity may become of importance as local structural variations such as juxtaluminal calcifications could contribute to plaque destabilization. However, uniform methods and cutoff values to discriminate vulnerable plaques from stable plaques are currently lacking.

Dedicated RF-based imaging systems are not widely available and require sophisticated image processing after data acquisition, thus limiting widespread implementation. Because of the differences in spatial resolution, I compared these two techniques to measure CCA-IMT in 136 stroke patients (Chapter 2). There was a slight difference in the methods: using routine B-mode US, we averaged the CCA-IMT over 10 $\mathrm{mm}$, whereas RF-based analysis used $12 \mathrm{M}$-lines covering $13.9 \mathrm{~mm}$. The two techniques were strongly correlated and had similar inter-measurement reproducibility. However, RF-based CCA-IMT was significantly smaller than B-mode CCA-IMT (mean difference of $45 \mu \mathrm{m}$ ). This finding has been replicated by others. ${ }^{75,76}$ In another study, we have demonstrated that B-mode and RF-based measurements of distension of the CAA had comparable precision and did not result in a systematic difference. ${ }^{77}$ These results support the premise that routinely acquired B-mode US can be used to study structural and functional vessel wall and plaque features as an alternative to the more advanced RF-based techniques.

An important limitation of most methods that depict the carotid artery in 2D is the inability to account for out-of-plane motion. Small movements, such as breathing, swallowing or slight translations of the US probe relative to the artery will result in disturbances of measurement of functional plaque properties. Most techniques image the carotid artery in the longitudinal vessel axis, with plaque being located at the anterior and/or posterior vessel wall. Instead, some authors image the carotid artery in the crosssectional plane. This also allows estimation of plaque strain at the lateral arterial walls, using compound imaging which employs multiple insonation angles. ${ }^{70}$ However, out-ofplane motion can even occur when using cross-sectional imaging. 3-dimensional carotid plaque imaging with a sufficient temporal resolution may provide a possible solution. 


\section{Methodological considerations}

Before moving to the clinical implications of the studies performed within the framework of this thesis, I will first discuss some methodological considerations, with an emphasize on study design, precision, internal and external validity.

\section{Study design and population}

With the exception of Chapter $\mathbf{6}$, all studies have been prospective cross-sectional cohort studies. Participants included in the studies were recruited from a hospital-based population. In Chapter 2 , Chapter 3 and Chapter 4 , we asked consent to participate from patients who were referred for carotid ultrasonography at the Department of Clinical Neurophysiology of the Maastricht University Medical Center (MUMC). Though the MUMC is an academic hospital, it also functions as a regional hospital to the city of Maastricht and its surroundings. As a result, the patients included in this study reflect a regular hospitalbased population. Most participants (72\%) were referred for US examination because of a recent ischemic stroke or (ocular) TIA. Mean age was 64 years with $60 \%$ being males (Chapter 2), or 66 years with $48 \%$ being males (Chapter 3 and Chapter 4). In Chapter 6 , we retrospectively reviewed Duplex US results for all patients (mean age 67 years, 54\% male; $74 \%$ with recent cerebrovascular events) referred for duplex US at the Department of Clinical Neurophysiology at the MUMC within a single year. Chapter 5 , Chapter 7 and Chapter 8 were based on the PARISK sub-studies, and all patients had a recent ischemic stroke or (ocular) TIA as this was an inclusion criteria. Patients were recruited from 13 regional and 4 participating academic hospitals. Compared to the other chapters, median age was higher (70-71 years) with a larger proportion of males (67-71\%).

\section{Precision}

Precision refers to the absence of random error and as such to the reliability of the study results. ${ }^{78}$ In general, larger study populations and better balance between groups with and without a primary outcome measure are likely to produce more precise estimates with smaller confidence intervals. In most chapters, we have reduced random error by inclusion of large populations (ranging from 136 to 810 subjects). The number of participants in Chapter $5(\mathrm{~N}=23)$ and Chapter $7(\mathrm{~N}=43)$ were rather small, though confidence intervals are sufficient (e.g. Table 7.3). For Chapter 8 it should be noticed that we took precautions to prevent overfitting of the prediction model, however, we could not yet externally validate PARISK-US score.

\section{Internal validity}

Validity refers to the lack of systematic error. Two types of bias may be relevant to the internal validity of the results in this thesis: selection bias and information bias. ${ }^{78}$

Selection bias occurs when participation is affected by the outcome of the study, i.e. 
people who participate are systematically different from people who do not participate in the study. For Chapter 2, Chapter 3 and Chapter 4, a participation log was kept. Between April and August 2007, 329 carotid US examinations were performed. A total of 160 patients agreed to participate in the study, whereas just 14 patients (4\%) declined participation. In the remaining 155 patients, measurements were not possible due to logistic reasons, mainly because the RF ultrasonography device was not available (in 103 subjects, which occurred randomly) or because the artery was beyond the maximal depth of the US device (35 patients, which is more likely to occur in obese patients). Based on these numbers, the risk of selection bias can be assumed to be small. For Chapter $\mathbf{6}$, we included all US examinations in a single year, so selection bias is not likely to have occurred here. For the remaining chapters, 238 participants were recruited from 17 regional and university hospitals over a period of 4.5 years. No formal participation log was registered, but it is likely that selection of participants may have occurred as the number of participants largely differs between the four study centers (Figure 8.1). Additionally, one of the exclusion criteria was severe comorbidity which will have resulted in the inclusion of less severely affected patients. This however reflects general practice in which patients with disabling ischemic stroke are unsuited to undergo carotid revascularization. Lastly, participants of the PARISK study underwent an extensive imaging protocol which may have made some patients reluctant to participate.

Information bias can occur when study parameters or outcomes are not properly defined or incorrectly measured. In all studies, US examinations were performed by experienced vascular technicians according to a standardized imaging protocol. All data was stored to allow offline analysis. In the PARISK study, risk of information bias was reduced by strict research protocols and standard operation procedures, including the collection and analysis of imaging. Readers of imaging data thoroughly trained on a consensus set and were only allowed to analyze imaging data if they had good interobserver agreement. All readers were blinded for clinical variables, outcome information and other imaging parameters. In the other chapters, we reduced information bias by using standard imaging protocols and uniform analyses. Nonetheless, information bias could not be completely prevented, e.g. blinding of readers was not possible.

\section{External validity}

External validity reflects the ability to generalize study results to a broader population. ${ }^{78}$ Overall, the participants included in the studies within this thesis are representative of the stroke population in general, with a mean age of $\sim 70$ years and the majority being male (see study design). The baseline data of the PARISK study are comparable to an ongoing CAPIAS study in Germany (median age 74 years, 68\% male). ${ }^{61}$ Because we mainly included patients with recent cerebrovascular disease, our results cannot be coarsely translated to patients with asymptomatic carotid artery disease or community-dwelling populations. 


\section{Causal interference}

All studies described in this thesis have a cross-sectional design, which limits us to describe associations instead of drawing definite conclusions on causality. Longitudinal data is needed to appreciate whether the found associations truly have a causal relationship. The PARISK longitudinal study will provide more insight whether the techniques introduced in this thesis are of use to incorporate in the risk stratification of individuals with recent cerebrovascular disease. Of course, the results will need to be replicated in studies in equivalent populations such as the CAPIAS study, to draw definite conclusions on the value of simultaneous assessment of structural and functional plaque properties.

\section{Clinical implications}

\section{Asymptomatic carotid artery stenosis}

The incidence of ischemic stroke has decreased over the past decades. Between 1990 and 2016, the global age-standardized incidence of ischemic stroke has declined with $8.1 \%{ }^{2}$ This decline is attributed to improved awareness and better treatment of modifiable risk factors for stroke, such as hypertension, hypercholesterolemia, diabetes mellitus, smoking cessation and lifestyle modification. Despite the declining incidence, the burden of stroke is still very high, with ischemic stroke being the second largest cause of death. This illustrates that targeting vascular risk factors for stroke is not sufficiently effective. As an alternative, screening for conditions that increase the risk of stroke has been advocated, such as atrial fibrillation. ${ }^{79}$ Should we also screen for the presence of asymptomatic carotid artery stenosis?

Atherosclerotic plaques slowly develop over the course of many years before they eventually become symptomatic. Consequently, moderate ( $\geq 50 \%$ stenosis) asymptomatic carotid artery plaques can be found in $4 \%$ of the population, whereas severe ( $\geq 70 \%$ stenosis) is present in $1.7 \%$ of the people. ${ }^{10}$ Prevalence of asymptomatic carotid artery stenosis is strongly associated with increasing age and male sex, thus up to $12.5 \%$ of elderly men ( $>70$ years) can have an asymptomatic carotid stenosis. ${ }^{10}$ In a recent network meta-analysis including 4 trials comparing CEA ( $n=2627$ patients) versus best medical treatment ( $n=2651$ patients), the risk of ipsilateral stroke was lower after CEA with pooled odds ratio of 0.59 ( $\mathrm{Cl}-95 \%$ 0.49-0.70). ${ }^{80}$ Still, the risk of ischemic stroke in patients with asymptomatic carotid artery stenosis has declined since the improved treatment of cardiovascular risk factors. In the Second Manifestations of Arterial disease (SMART) study, the annual ipsilateral stroke risk was $0.4 \%$ for $50-99 \%$ stenosis, and $0.5 \%$ for $70-99 \%$ stenosis. $^{81}$ Selecting asymptomatic individuals for carotid revascularization only on the degree of stenosis will not become a (cost-)effective strategy. Hence, the current European guideline on carotid artery disease does not advocate population screening for asymptomatic carotid artery stenosis. ${ }^{4}$ 


\section{Beyond the degree of stenosis}

Instead, we need to take a better look at the arteries we intend to treat, similar to the way we treat other diseases such as hypertension, where it is common practice to perform measurements of disease severity. The above-mentioned European guideline suggests cautiously that in patients in whom an asymptomatic 60-99\% stenosis is found, carotid revascularization may be considered if one or more imaging characteristics associated with an increased risk of ipsilateral stroke are present. ${ }^{4}$ Most of the suggested imaging characteristics include structural properties, such as IPH on carotid MRI, plaque echogenicity, large plaque size of juxtaluminal dark areas suggestive of plaque ulcerations. ${ }^{82}$ However, when using a single marker, associations with future risk of stroke are too weak to identify the individuals at high risk of future stroke. For example, in a meta-analysis of 7 studies with 7557 asymptomatic subjects with $>50 \%$ stenosis, plaque echogenicity was predictive of future ipsilateral stroke (relative risk 2.6, 95\%-Cl 1.5-4.6) during a mean follow-up of 37 months. ${ }^{83}$ As an alternative, IPH was strongly associated with the risk of future stroke (hazard ratio 7.9, 95\%-Cl 1.3-47.6) in the before-mentioned individual patient data meta-analysis, but the number of included subjects was small. ${ }^{6}$

Consequently, it is likely that a combination of techniques should be used for risk stratification. Ideally, this imaging strategy should include the main features of plaque destabilization (i.e. plaque composition, inflammation and biomechanical forces working on the plaque) to fully appreciate the risk of future events. The importance of a combined approach does not only hold true for asymptomatic individuals, since risk stratification in recently symptomatic patients and moderate-to-severe stenosis is similarly inaccurate. ${ }^{5}$

In this thesis, I demonstrate that simultaneously investigating structural and functional plaque properties in patients with recent cerebrovascular disease is feasible and reproducible, and that it can be used in a diagnostic prediction score to reliably identify patients with IPH, being at an increased risk of stroke recurrence. Additionally, analysis of functional plaque behavior may have additional value on top of structural plaque properties. The PARISK-US score first needs external validation before it can be applicated in clinical practice, whereas the use of simultaneously investigating structural and functional plaque properties to predict symptom recurrence also requires further research.

\section{Treatment of plaque destabilization}

Besides carotid revascularization, alternative approaches may exist to reduce the risk of future stroke in patients with carotid artery stenosis. Medical treatment can not only reduce the risk of subsequent stroke through improvements in the risk factor profile, such as reduction of cholesterol levels or hypertension control, but may also transform rupture-prone plaques to a more stable configuration.

Plaque composition can be altered by use of high-dose statins. In a meta-analysis of 10 studies which had 361 patients, statin treatment for more than 12 months was 
associated with a significant decrease in LRNC volume on carotid MRI (weighted mean difference $\left.9.9 \mathrm{~mm}^{3}, 95 \%-\mathrm{Cl} 0.8-18.9 \mathrm{~mm}^{3}\right) .{ }^{84} \mathrm{~A}$ study that was published after this metaanalysis showed that in the 1740 participants from the Rotterdam study, high dosage of statins was associated with a lower prevalence of LRNC on carotid MRI. ${ }^{85}$ Besides the effect on plaque composition, statins may also reduce plaque instability by reducing inflammation. In a meta-analysis of 9 studies including 566 patients with a mean followup duration of 7 months, plaque echogenicity was improved after statin therapy with a pooled weighted mean difference of $29 \%(95 \%-\mathrm{Cl} 22-36 \%){ }^{86}$ This treatment effect occurred independent from changes in cholesterol levels, but instead was related to changes in high-sensitive CRP. To conclude, in subjects with vulnerable plaques, high-dose statins may be considered to improve plaque composition to a more favorable phenotype and reduce plaque inflammation and the lipid-rich necrotic core size, irrespective of the serum cholesterol levels.

Hemodynamic forces involved in plaque destabilization can also be altered to possibly improve the risk of plaque rupture. Local pulse pressure is the primary hemodynamic force contributing to rupture of culprit lesions. Increased pulse pressure has been shown to associated with an increased risk of stroke (pooled HR for an increase of $10 \mathrm{mmHg}: 1.05$, $95 \%-\mathrm{Cl} 1.03-1.07$ ) in a meta-analysis of 8 studies including 126,067 patients. ${ }^{34}$ Specifically targeting pulse pressure may be of benefit, for example by means of nitrates, though studies are currently lacking. ${ }^{87}$

\section{Future perspectives}

Non-invasive imaging for risk stratification in recently symptomatic patients

Between August 2010 and December 2014, 244 patients have been included in the prospective, observational multicenter Plaque At RISK study (PARISK, NCT01208025). ${ }^{88}$ Primary objective of the PARISK study is to investigate whether (a combination of) noninvasive imaging techniques can help to improve risk stratification of patient with recent ischemic stroke or TIA in the anterior circulation and ipsilateral mild to moderate carotid artery stenosis. Patients were included within three months of the last cerebrovascular ischemic event. Carotid artery plaque had to have be at least $2 \mathrm{~mm}$ but less than $70 \%$ luminal stenosis. Regular stroke work-up had to exclude cardiac sources of embolism or clotting disorders as a cause of the index stroke.

At baseline, patients underwent 3T multisequence carotid and brain MRI, multidetector-row CT-angiography, carotid ultrasonography, blood sampling to study biomarkers, and ambulatory transcranial Doppler recordings of the ipsilateral middle cerebral artery to detect microemboli. Data from these baseline studies have been used to cross-sectionally investigate associations between the different clinical and imaging parameters to improve our understanding of plaque pathogenesis. Chapter 5 , Chapter 7 and Chapter 8 used baseline data from the PARISK study. Data from the PARISK study 
could be used to validate the findings in Chapter $\mathbf{6}$, as to determine whether carotid US is less accurate in the extremities of age.

Since conclusions on causality cannot been drawn from cross-sectional data, longitudinal data analysis is awaited. Patients were followed at regular intervals to detect recurrent stroke and other cardiovascular events. At two years follow-up, all patients underwent repeated brain MRI to also detect clinically silent ischemic events. Primary study endpoint of the longitudinal analysis of the PARISK study is the combined occurrence of recurrent ischemic stroke and/or new ischemic brain lesions on follow-up brain MRI. As the prespecified number of included participants (300 participants) was not reached and the number of recurrent events was lower than anticipated (sample size was calculated for an event rate of $2.5 \%$ per year), the clinical follow-up was extended to five years. In December 2019, the last follow-up interviews have been finished. The results of the PARISK longitudinal study investigating the value of non-invasive imaging for the prediction stroke recurrence are expected in early 2021. Together with the expected simultaneous publication of the comparable CAPIAS study (NCT01284933)61, this will help to appreciate whether non-invasive carotid imaging can improve risk stratification. Data from this cohort study can also be used to externally validate the PARISK-US score, which was developed in Chapter 8.

To determine whether plaque imaging can guide treatment decisions, a randomized clinical trial is needed in which patients with low risk of future stroke but with a vulnerable plaque features on imaging would be randomized between carotid revascularization and best medical treatment. Such a trial has not yet been started. Recently, the value of carotid plaque imaging was investigated as part of the second European Carotid Surgery Trial (ECST-2, ISRCTN97744893). The ECST-2 was a multicenter, prospective trial with blinded outcome assessment, that randomized patients with carotid artery stenosis of at least $50 \%$ with $<20 \%$ risk of future stroke between carotid revascularization with best medical treatment versus best medical treatment alone. Patients were to be followed for at least 5 years to determine the risk stroke recurrence between the groups. A selective group of patients underwent carotid MRI in dedicated centers to investigate the predictive value of plaque morphology to aid in the risk stratification, but plaque imaging was not a variable used to stratify treatment allocation. Moreover, the ECST-2 has been prematurely halted; the results are currently awaited.

\section{Insight in plaque destabilization from longitudinal imaging cohorts.}

To better understand the events leading to plaque destabilization, plaque rupture and subsequent stroke recurrence, in vivo serial imaging studies are needed. Few such studies exist using carotid MRI to investigate changes in plaque morphology over time. In 92 patients with cerebrovascular events, change in plaque morphology was limited over a 1 year time period. At baseline, 20 patients had IPH. In 4 patients, IPH disappeared at follow-up whereas in 3 patients new IPH had developed. ${ }^{89}$ No attempt is made to assess 
which factors contribute to the development of IPH, as numbers were too small. A second study investigates the change in plaque components over a 4-year time period in 198 subjects included in the population-based Rotterdam study. In contrast to the first paper, incident IPH occurred in $19 \%$ of subjects. Severe hypertension (odds ratio $4.7,95 \%-\mathrm{Cl} 1.5-$ 14.8) and use of antihypertensive drugs (odds ratio 3.9, 95\%-Cl 1.9-7.9) were associated with incident IPH..$^{90}$ This observation supports the hypothesis that biomechanical forces are involved in the development of new IPH.

Unfortunately, neither of these two studies included functional plaque assessment. As part of the longitudinal PARISK project, the first 150 participants underwent repeated carotid MRI at two years. This will allow to correlate incident IPH and progression of existing IPH with baseline clinical and imaging factors, most important being blood pressure parameters as well as functional plaque properties. These data are expected to increase our understanding of the role of hemodynamics and biomechanics in plaque destabilization, and can possibly directly link biomechanical load to the development of recurrent strokes. A limitation to the PARISK study is that patients were included 46 days (IQR 31-67 days) after their initial stroke or TIA, whereas stroke recurrences tend to occur early after index stroke. In CAPIAS, patients are recruited within 7 days of stroke onset, which may increase the number of stroke recurrences. Still, carotid MRI will be repeated at 1 year after baseline in CAPIAS, thus reducing incident IPH at follow-up.

In case results from the PARISK study indicate that biomechanical stress on the plaque is important in plaque rupture with subsequent embolization, this may be further explored in studies aimed to reduce plaque strain. A phase-II clinical trial could explore the effect of specific drugs at reducing the biomechanical load on the development of new IPH and should be used as an intermediate step before studying the effect of such medication on stroke recurrence in a phase-III randomized clinical trial.

\section{Identification of individuals at high risk of future stroke}

As mentioned at the discussion of the clinical implications of this thesis, screening for asymptomatic carotid stenosis is not advocated at present since it remains difficult to identify individuals who are at increased risk of stroke. Large, population-based studies are needed to address the use of carotid plaque characterization for individualized risk stratification. Two such studies exist within the Netherlands and have already included carotid US and/or MRI as part of the baseline and follow-up examinations. The Rotterdam study is a prospective cohort study that started in the 1990s and currently compromises nearly 14,000 inhabitants of a specific district in the city of Rotterdam. ${ }^{91}$ As part of this project, over a 1,000 participants underwent 1.5T carotid MRI from 2007 onwards, after US screening had demonstrated the presence of carotid plaque of at least $2.5 \mathrm{~mm}$. The Maastricht study started enrollment of 10,000 inhabitants from the Maastricht region in 2010. ${ }^{92}$ Whereas its main focus is to improve understanding of the etiology of type 2 diabetes, it also includes carotid US. Either study could assess the value of structural 
and functional plaque properties to predict the occurrence of future strokes. As an intermediate step, the PARISK-US score could be adjusted to also predict the presence of $\mathrm{IPH}$ in asymptomatic individuals.

One might speculate that in the future it will become less relevant to know whether an individual has had recent symptoms. Instead, thorough assessment of plaque structural and functional properties, together with evaluation of plaque inflammation, may facilitate the individual prediction of future stroke risk and guide treatment decisions to specifically target the plaque at risk.

\section{Concluding remarks}

In this thesis, I demonstrate that simultaneously investigating structural and functional vessel wall and plaque properties in patients with recent cerebrovascular disease is feasible and reproducible. Moreover, structural and functional plaque properties can be used in a diagnostic prediction score to reliably identify patients with IPH, being at an increased risk of stroke recurrence. Additionally, analysis of functional plaque behavior may have additional value besides plaque morphological assessment. 


\section{References}

1. Spence JD. Carotid plaque measurement is superior to IMT; invited editorial comment on: Carotid plaque, compared with carotid intima-media thickness, more accurately predicts coronary artery disease events: A meta-analysis. Atherosclerosis. 2012;220:34-35

2. Johnson CO, Nguyen M, Roth GA, Nichols E, Alam T, Abate D, et al. Global, regional, and national burden of stroke, 1990-2016: A systematic analysis for the global burden of disease study 2016. The Lancet Neurology. 2019;18:439-458

3. Opara JA, Jaracz K. Quality of life of post-stroke patients and their caregivers. Journal of medicine and life. 2010;3:216-220

4. Naylor AR, Ricco JB, de Borst GJ, Debus S, de Haro J, Halliday A, et al. Management of atherosclerotic carotid and vertebral artery disease: 2017 clinical practice guidelines of the European society for vascular surgery (ESVS). European journal of vascular and endovascular surgery. 2018;55:3-81

5. Rothwell PM, Eliasziw M, Gutnikov SA, Fox AJ, Taylor DW, Mayberg MR, et al. Analysis of pooled data from the randomised controlled trials of endarterectomy for symptomatic carotid stenosis. Lancet. 2003;361:107-116

6. Schindler A, Schinner R, Altaf N, Hosseini AA, Simpson RJ, Esposito-Bauer L, et al. Prediction of stroke risk by detection of hemorrhage in carotid plaques: Meta-analysis of individual patient data. JACC Cardiovascular Imaging. 2020;13:395-406

7. Ntaios G, Perlepe K, Sirimarco G, Strambo D, Eskandari A, Karagkiozi E, et al. Carotid plaques and detection of atrial fibrillation in embolic stroke of undetermined source. Neurology. 2019;92:e2644-e2652

8. Hart RG, Catanese L, Perera KS, Ntaios G, Connolly SJ. Embolic stroke of undetermined source: A systematic review and clinical update. Stroke. 2017;48:867-872

9. Kamtchum-Tatuene J, Wilman A, Saqqur M, Shuaib A, Jickling GC. Carotid plaque with high-risk features in embolic stroke of undetermined source: Systematic review and meta-analysis. Stroke. 2020;51:311-314

10. de Weerd M, Greving JP, de Jong AW, Buskens E, Bots ML. Prevalence of asymptomatic carotid artery stenosis according to age and sex: Systematic review and metaregression analysis. Stroke. 2009;40:1105-1113

11. Abbott AL, Paraskevas KI, Kakkos SK, Golledge J, Eckstein HH, Diaz-Sandoval LJ, et al. Systematic review of guidelines for the management of asymptomatic and symptomatic carotid stenosis. Stroke. 2015;46:3288-3301

12. DICA jaarrapportage 2018.

13. Eyding J, Kitzrow M, Krogias C, Reimann G, Weber R, Weimar C, et al. Versorgungsrealität von Karotisstenosen in Deutschland: Forderung und Wirklichkeit im internationalen Vergleich und im Lichte der Aktuellen S3-Leitlinie. Der Nervenarzt. 2015;86:1261-1267

14. Chapter 8 - Ultrasound. In: Nichols WW, O'Rourke MF, Vlachopoulos C, eds. Mcdonald's blood flow in arteries: Theoretical, experimental and clinical principles. London: Hodder Arnold; 2011:177-194.

15. Reneman RS, Arts T, Hoeks AP. Wall shear stress - an important determinant of endothelial cell function and structure--in the arterial system in vivo. Discrepancies with theory. Journal of Vascular Research. 2006;43:251-269

16. Spencer MP, Reid JM. Quantitation of carotid stenosis with continuous-wave (C-W) doppler 
ultrasound. Stroke. 1979;10:326-330

17. Slager CJ, Wentzel JJ, Gijsen FJ, Thury A, van der Wal AC, Schaar JA, et al. The role of shear stress in the destabilization of vulnerable plaques and related therapeutic implications. Nature clinical practice. Cardiovascular medicine. 2005;2:456-464

18. Chappell DC, Varner SE, Nerem RM, Medford RM, Alexander RW. Oscillatory shear stress stimulates adhesion molecule expression in cultured human endothelium. Circulation Research. 1998;82:532539

19. Wang Y, Qiu J, Luo S, Xie X, Zheng Y, Zhang K, et al. High shear stress induces atherosclerotic vulnerable plaque formation through angiogenesis. Regenerative Biomaterials. 2016;3:257-267

20. Saris A, Hansen HHG, Fekkes S, Menssen J, Nillesen MM, de Korte CL. In vivo blood velocity vector imaging using adaptive velocity compounding in the carotid artery bifurcation. Ultrasound in medicine \& biology. 2019;45:1691-1707

21. Kwee RM, Truijman MT, Mess WH, Teule GJ, ter Berg JW, Franke CL, et al. Potential of integrated [18F] fluorodeoxyglucose positron-emission tomography/CT in identifying vulnerable carotid plaques. American journal of neuroradiology. 2011;32:950-954

22. Voo S, Kwee RM, Sluimer JC, Schreuder FH, Wierts R, Bauwens M, et al. Imaging intraplaque inflammation in carotid atherosclerosis with 18F-fluorocholine positron emission tomographycomputed tomography: Prospective study on vulnerable atheroma with immunohistochemical validation. Circulation. Cardiovascular imaging. 2016;9

23. Hoeks AP, Kooi ME. Wear and tear. JACC Cardiovascular Imaging. 2011;4:478-480

24. Pei X, Wu B, Tang TY, Gillard JH, Li ZY. Fatigue crack growth under pulsatile pressure and plaque rupture. JACC Cardiovascular Imaging. 2014;7:738-740

25. Li ZY, Taviani V, Tang T, Sadat U, Young V, Patterson A, et al. The mechanical triggers of plaque rupture: Shear stress vs pressure gradient. British Journal of Radiology. 2009;82 Spec No 1:S39-45

26. Hoeks AP, Reesink KD, Hermeling E, Reneman RS. Local blood pressure rather than shear stress should be blamed for plaque rupture. Journal of the Americal College of Cardiology. 2008;52:11071109

27. Takaya N, Yuan C, Chu B, Saam T, Polissar NL, Jarvik GP, et al. Presence of intraplaque hemorrhage stimulates progression of carotid atherosclerotic plaques: $\mathrm{A}$ high-resolution magnetic resonance imaging study. Circulation. 2005;111:2768-2775

28. Daemen MJ, Ferguson MS, Gijsen FJ, Hippe DS, Kooi ME, Demarco K, et al. Carotid plaque fissure: An underestimated source of intraplaque hemorrhage. Atherosclerosis. 2016;254:102-108

29. Cicha I, Worner A, Urschel K, Beronov K, Goppelt-Struebe M, Verhoeven E, et al. Carotid plaque vulnerability: A positive feedback between hemodynamic and biochemical mechanisms. Stroke. 2011;42:3502-3510

30. Fagerberg B, Ryndel M, Kjelldahl J, Akyurek LM, Rosengren L, Karlstrom L, et al. Differences in lesion severity and cellular composition between in vivo assessed upstream and downstream sides of human symptomatic carotid atherosclerotic plaques. Journal of Vascular Research. 2010;47:221-230

31. Lovett JK, Rothwell PM. Site of carotid plaque ulceration in relation to direction of blood flow: An angiographic and pathological study. Cerebrovascular diseases. 2003;16:369-375

32. Crombag GA, Schreuder FH, Benali F, van Dam-Nolen HK, Liem MI, Lucci C, et al. Proximal region of carotid atherosclerotic plaque shows more intraplaque hemorrhage: The plaque at risk (PARISK) study. In: Crombag GA. PhD thesis. Magnetic resonance imaging of carotid intraplaque hemorrhage 
and microvasculature. Maastricht: Maastricht University; 2019:61-75.

33. Selwaness M, van den Bouwhuijsen QJ, Verwoert GC, Dehghan A, Mattace-Raso FU, Vernooij M, et al. Blood pressure parameters and carotid intraplaque hemorrhage as measured by magnetic resonance imaging: The Rotterdam study. Hypertension. 2013;61:76-81

34. Liu FD, Shen XL, Zhao R, Tao XX, Wang S, Zhou JJ, et al. Pulse pressure as an independent predictor of stroke: A systematic review and a meta-analysis. Clinical research in cardiology. 2016;105:677-686

35. O'Leary DH, Polak JF, Kronmal RA, Manolio TA, Burke GL, Wolfson SK, Jr. Carotid-artery intima and media thickness as a risk factor for myocardial infarction and stroke in older adults. Cardiovascular health study collaborative research group. The New England journal of medicine. 1999;340:14-22

36. Touboul PJ, Elbaz A, Koller C, Lucas C, Adrai V, Chedru F, et al. Common carotid artery intima-media thickness and brain infarction: The etude du profil genetique de l'infarctus cerebral (GENIC) casecontrol study. The genic investigators. Circulation. 2000;102:313-318

37. Den Ruijter HM, Peters SA, Anderson TJ, Britton AR, Dekker JM, Eijkemans MJ, et al. Common carotid intima-media thickness measurements in cardiovascular risk prediction: A meta-analysis. JAMA. 2012;308:796-803

38. Bots ML, Hofman A, Grobbee DE. Increased common carotid intima-media thickness. Adaptive response or a reflection of atherosclerosis? Findings from the rotterdam study. Stroke. 1997;28:24422447

39. van den Munckhof ICL, Jones H, Hopman MTE, de Graaf J, Nyakayiru J, van Dijk B, et al. Relation between age and carotid artery intima-medial thickness: A systematic review. Clinical Cardiology. 2018;41:698-704

40. Meinders JM, Kornet L, Hoeks AP. Assessment of spatial inhomogeneities in intima media thickness along an arterial segment using its dynamic behavior. American Journal of Physiology and Heart Circulation Physiology. 2003;285:H384-391

41. Touboul PJ, Hennerici MG, Meairs S, Adams H, Amarenco P, Bornstein N, et al. Mannheim carotid intima-media thickness and plaque consensus (2004-2006-2011). An update on behalf of the advisory board of the 3rd, 4th and 5th watching the risk symposia, at the 13th, 15th and 20th European stroke conferences, Mannheim, Germany, 2004, Brussels, Belgium, 2006, and Hamburg, Germany, 2011. Cerebrovascular diseases. 2012;34:290-296

42. Saba L, Meiburger KM, Molinari F, Ledda G, Anzidei M, Acharya UR, et al. Carotid IMT variability and its validation in symptomatic versus asymptomatic Italian population: Can this be a useful index for studying symptomaticity? Echocardiography. 2012;29:1111-1119

43. Steinbuch J, van Dijk AC, Schreuder F, Truijman MTB, de Rotte AAJ, Nederkoorn PJ, et al. High spatial inhomogeneity in the intima-media thickness of the common carotid artery is associated with a larger degree of stenosis in the internal carotid artery: The PARISK study. Ultraschall in der Medizin. 2017;38:523-529

44. Reneman RS, Meinders JM, Hoeks AP. Non-invasive ultrasound in arterial wall dynamics in humans: What have we learned and what remains to be solved. European heart journal. 2005;26:960-966

45. Zhong Q, Hu MJ, Cui YJ, Liang L, Zhou MM, Yang YW, et al. Carotid-femoral pulse wave velocity in the prediction of cardiovascular events and mortality: An updated systematic review and metaanalysis. Angiology. 2018;69:617-629

46. van Sloten TT, Sedaghat S, Laurent S, London GM, Pannier B, Ikram MA, et al. Carotid stiffness is associated with incident stroke: A systematic review and individual participant data meta-analysis. 
Journal of the Americal College of Cardiology. 2015;66:2116-2125

47. Steinbuch J, Schreuder FH, Truijman MT, Hendrikse J, Nederkoorn PJ, Van der Lugt A, et al. Spatial inhomogeneity of diastolic-systolic risetime of the distension waveform in the common carotid artery is associated with lipid-rich necrotic corse in distal plaques: The plaque at risk (PARISK) study. In: Steinbuch J. PhD Thesis. In vivo ultrasound assessment of carotid artery walls and plaques: Integrating morphological and mechanical characteristics. Maastricht: Maastricht University; 2017:99-113.

48. Rundek T, Arif H, Boden-Albala B, Elkind MS, Paik MC, Sacco RL. Carotid plaque, a subclinical precursor of vascular events: The northern Manhattan study. Neurology. 2008;70:1200-1207

49. Mathiesen EB, Johnsen SH, Wilsgaard T, Bonaa KH, Lochen ML, Njolstad I. Carotid plaque area and intima-media thickness in prediction of first-ever ischemic stroke: A 10-year follow-up of 6584 men and women: The Tromso study. Stroke. 2011;42:972-978

50. Sillesen H, Sartori S, Sandholt B, Baber U, Mehran R, Fuster V. Carotid plaque thickness and carotid plaque burden predict future cardiovascular events in asymptomatic adult americans. European Heart Journal of Cardiovascular Imaging. 2018;19:1042-1050

51. el-Barghouty N, Geroulakos G, Nicolaides A, Androulakis A, Bahal V. Computer-assisted carotid plaque characterisation. European journal of vascular and endovascular surgery. 1995;9:389-393

52. El-Barghouty NM, Levine T, Ladva S, Flanagan A, Nicolaides A. Histological verification of computerised carotid plaque characterisation. European journal of vascular and endovascular surgery. 1996;11:414-416

53. Tegos TJ, Sohail M, Sabetai MM, Robless P, Akbar N, Pare G, et al. Echomorphologic and histopathologic characteristics of unstable carotid plaques. American journal of neuroradiology. 2000;21:1937-1944

54. Gronholdt ML, Nordestgaard BG, Schroeder TV, Vorstrup S, Sillesen H. Ultrasonic echolucent carotid plaques predict future strokes. Circulation. 2001;104:68-73

55. Sztajzel R, Momjian S, Momjian-Mayor I, Murith N, Djebaili K, Boissard G, et al. Stratified grayscale median analysis and color mapping of the carotid plaque: Correlation with endarterectomy specimen histology of 28 patients. Stroke. 2005;36:741-745

56. Mitchell CC, Stein JH, Cook TD, Salamat S, Wang X, Varghese T, et al. Histopathologic validation of grayscale carotid plaque characteristics related to plaque vulnerability. Ultrasound in medicine \& biology. 2017;43:129-137

57. Griffin MB, Kyriacou E, Pattichis C, Bond D, Kakkos SK, Sabetai M, et al. Juxtaluminal hypoechoic area in ultrasonic images of carotid plaques and hemispheric symptoms. Journal of vascular surgery. 2010;52:69-76

58. Grant EG, Benson CB, Moneta GL, Alexandrov AV, Baker JD, Bluth El, et al. Carotid artery stenosis: Gray-scale and doppler US diagnosis - Society of radiologists in ultrasound consensus conference. Radiology. 2003;229:340-346

59. Massara M, Notarstefano S, Gerardi P, Prunella R, Impedovo G. Unstable atherosclerotic plaque in the common carotid artery: Diagnosis and treatment strategy. Seminars in Vascular Surgery. 2018;31:88-90

60. Nichols WW, O'Rourke MF, Vlachopoulos C. Mcdonald's blood flow in arteries: Theoretic, experimental, and clinical principles. London: Hodder Arnold; 2011.

61. Bayer-Karpinska A, Schwarz F, Wollenweber FA, Poppert H, Boeckh-Behrens T, Becker A, et al. The 
carotid plaque imaging in acute stroke (CAPIAS) study: Protocol and initial baseline data. BMC neurology. 2013;13:201

62. Nederveen AJ, Avril S, Speelman L. MRI strain imaging of the carotid artery: Present limitations and future challenges. Journal of Biomechanics. 2014;47:824-833

63. Bang J, Dahl T, Bruinsma A, Kaspersen JH, Nagelhus Hernes TA, Myhre HO. A new method for analysis of motion of carotid plaques from rf ultrasound images. Ultrasound in medicine \& biology. 2003;29:967-976

64. Dahl T, Bang J, Ushakova A, Lydersen S, Myhre HO. Parameters describing motion in carotid artery plaques from ultrasound examination: A reproducibility study. Ultrasound in medicine \& biology. 2004;30:1133-1143

65. Paini A, Boutouyrie P, Calvet D, Zidi M, Agabiti-Rosei E, Laurent S. Multiaxial mechanical characteristics of carotid plaque: Analysis by multiarray echotracking system. Stroke. 2007;38:117-123

66. Beaussier H, Naggara O, Calvet D, Joannides R, Guegan-Massardier E, Gerardin E, et al. Mechanical and structural characteristics of carotid plaques by combined analysis with echotracking system and MR imaging. JACC Cardiovascular Imaging. 2011;4:468-477

67. Naim C, Cloutier G, Mercure E, Destrempes F, Qin Z, El-Abyad W, et al. Characterisation of carotid plaques with ultrasound elastography: Feasibility and correlation with high-resolution magnetic resonance imaging. European radiology. 2013;23:2030-2041

68. Cloutier G, Cardinal MR, Ju Y, Giroux MF, Lanthier S, Soulez G. Carotid plaque vulnerability assessment using ultrasound elastography and echogenicity analysis. American journal of roentgenology. 2018;211:847-855

69. Huang C, Pan X, He Q, Huang M, Huang L, Zhao X, et al. Ultrasound-based carotid elastography for detection of vulnerable atherosclerotic plaques validated by magnetic resonance imaging. Ultrasound in medicine \& biology. 2016;42:365-377

70. Hansen HH, de Borst GJ, Bots ML, Moll FL, Pasterkamp G, de Korte CL. Validation of noninvasive in vivo compound ultrasound strain imaging using histologic plaque vulnerability features. Stroke. 2016;47:2770-2775

71. Shi H, Mitchell CC, McCormick M, Kliewer MA, Dempsey RJ, Varghese T. Preliminary in vivo atherosclerotic carotid plaque characterization using the accumulated axial strain and relative lateral shift strain indices. Physics in Medicine and Biology. 2008;53:6377-6394

72. Meshram NH, Mitchell CC, Wilbrand SM, Dempsey RJ, Varghese T. In vivo carotid strain imaging using principal strains in longitudinal view. Biomedical Physics and Engineering Express. 2019;5

73. Khan AA, Sikdar S, Hatsukami T, Cebral J, Jones M, Huston J, et al. Noninvasive characterization of carotid plaque strain. Journal of vascular surgery. 2017;65:1653-1663

74. Hansen KL, Nielsen MB, Jensen JA. Vector velocity estimation of blood flow - a new application in medical ultrasound. Ultrasound. 2017;25:189-199

75. Naredo E, Moller I, Gutierrez M, Bong DA, Cobo T, Corominas H, et al. Multi-examiner reliability of automated radio frequency-based ultrasound measurements of common carotid intima-media thickness in rheumatoid arthritis. Rheumatology. 2011;50:1860-1864

76. El Jalbout R, Cloutier G, Cardinal MR, Henderson M, Lapierre C, Soulez G, et al. Carotid artery intima-media thickness measurement in children with normal and increased body mass index: $A$ comparison of three techniques. Pediatric radiology. 2018;48:1073-1079

77. Steinbuch J, Hoeks AP, Hermeling E, Truijman MT, Schreuder FH, Mess WH. Standard B-mode 
ultrasound measures local carotid artery characteristics as reliably as radiofrequency phase tracking in symptomatic carotid artery patients. Ultrasound in medicine \& biology. 2016;42:586-595

78. Carlson MD, Morrison RS. Study design, precision, and validity in observational studies. Journal of Palliative Medicine. 2009;12:77-82

79. Feigin VL, Norrving B, George MG, Foltz JL, Roth GA, Mensah GA. Prevention of stroke: A strategic global imperative. Nature reviews. Neurology. 2016;12:501-512

80. Barkat M, Roy I, Antoniou SA, Torella F, Antoniou GA. Systematic review and network meta-analysis of treatment strategies for asymptomatic carotid disease. Scientific reports. 2018;8:4458

81. den Hartog AG, Achterberg S, Moll FL, Kappelle LJ, Visseren FL, van der Graaf Y, et al. Asymptomatic carotid artery stenosis and the risk of ischemic stroke according to subtype in patients with clinical manifest arterial disease. Stroke. 2013;44:1002-1007

82. Naylor AR. Time to rethink management strategies in asymptomatic carotid artery disease. Nature Reviews Cardiology. 2011;9:116-124

83. Gupta A, Kesavabhotla K, Baradaran H, Kamel H, Pandya A, Giambrone AE, et al. Plaque echolucency and stroke risk in asymptomatic carotid stenosis: A systematic review and meta-analysis. Stroke. 2015;46:91-97

84. Brinjikji W, Lehman VT, Kallmes DF, Rabinstein AA, Lanzino G, Murad MH, et al. The effects of statin therapy on carotid plaque composition and volume: A systematic review and meta-analysis. Journal of Neuroradiology. 2017;44:234-240

85. Mujaj B, Bos D, Selwaness M, Leening MJG, Kavousi M, Wentzel JJ, et al. Statin use is associated with carotid plaque composition: The Rotterdam study. International journal of cardiology. 2018;260:213218

86. Ibrahimi P, Jashari F, Bajraktari G, Wester P, Henein MY. Ultrasound assessment of carotid plaque echogenicity response to statin therapy: A systematic review and meta-analysis. International Journal of Molecular Science. 2015;16:10734-10747

87. Fok H, Jiang B, Clapp B, Chowienczyk P. Regulation of vascular tone and pulse wave velocity in human muscular conduit arteries: Selective effects of nitric oxide donors to dilate muscular arteries relative to resistance vessels. Hypertension. 2012;60:1220-1225

88. Truijman MT, Kooi ME, van Dijk AC, de Rotte AA, van der Kolk AG, Liem MI, et al. Plaque at risk (PARISK): Prospective multicenter study to improve diagnosis of high-risk carotid plaques. International journal of stroke. 2014;9:747-754

89. Kwee RM, Truijman MT, van Oostenbrugge RJ, Mess WH, Prins MH, Franke CL, et al. Longitudinal MRI study on the natural history of carotid artery plaques in symptomatic patients. PloS one. 2012;7:e42472

90. Pletsch-Borba L, Selwaness M, van der Lugt A, Hofman A, Franco OH, Vernooij MW. Change in carotid plaque components: A 4-year follow-up study with serial MR imaging. JACC Cardiovascular Imaging. 2018;11:184-192

91. Ikram MA, Brusselle GGO, Murad SD, van Duijn CM, Franco OH, Goedegebure A, et al. The Rotterdam study: 2018 update on objectives, design and main results. European Journal of Epidemiology. 2017;32:807-850

92. Schram MT, Sep SJ, van der Kallen CJ, Dagnelie PC, Koster A, Schaper N, et al. The Maastricht study: An extensive phenotyping study on determinants of type 2 diabetes, its complications and its comorbidities. European Journal of Epidemiology. 2014;29:439-451 




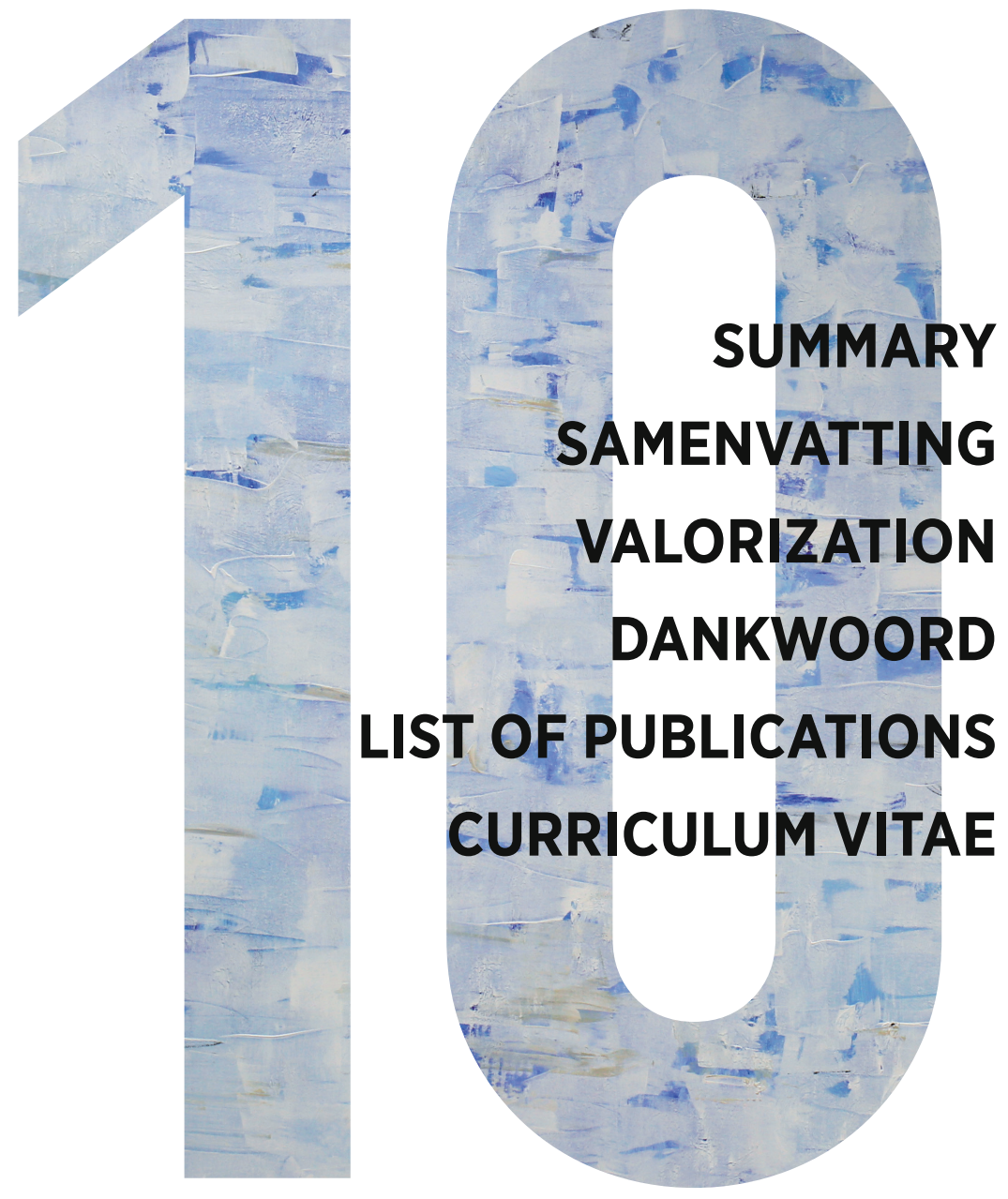





\section{Summary}

Ischemic stroke refers to the process where arterial occlusion results in damage to the brain area supplied by the vessel involved, leading to neurological dysfunction. Ischemic stroke is a major burden on health care around the world. In 2016, an estimated 13.7 million people had an ischemic stroke of whom 5.5 million died. Over 29 thousand patients were admitted because an ischemic stroke in the Netherlands in 2018.

Atherosclerotic plaque formation at the carotid artery bifurcation is an important cause for ischemic stroke. Rupture of the fibrotic cap overlying the plaque exposes the thrombogenic plaque content to the circulating blood causing luminal thrombosis, from which emboli may travel to the cerebral circulation. Hence, all patients with ischemic stroke undergo imaging of the carotid arteries to screen for the presence of carotid artery stenosis. To estimate the risk of future stroke recurrence and guide treatment decisions, current guidelines advocate to use the severity of luminal stenosis as the primarily method to select recently symptomatic patients for carotid revascularization. However, risk stratification fails to adequately identify patients who will most likely to benefit from carotid revascularization since luminal stenosis is limited in its predictive value for stroke recurrence. Improving our understanding in the processes ultimately resulting in plaque rupture might help to improve patient selection. Key pathophysiological factors that are thought to contribute to plaque rupture are plaque composition, plaque inflammation and biomechanical forces working on the plaque.

Carotid ultrasonography (US) can be used as a non-invasive technique to simultaneously study plaque structural and functional properties in patients with recent cerebrovascular disease. This thesis aimed to contribute to the development and validation of US techniques which enable in vivo assessment of structural and functional vessel wall and plaque properties, as to gain more insight in the events resulting in plaque rupture. More specifically, this thesis addressed the following objectives:

- To evaluate different ultrasonography techniques investigating the structural and functional properties of common carotid artery (CCA) vessel wall and atherosclerotic plaque in the CCA;

- To gain insight in the interplay between structural and functional properties of CCA vessel wall and more distally located carotid plaque at the carotid bifurcation;

- To use these ultrasonography techniques in the anatomically more complex carotid artery bifurcation to study the simultaneous assessment of structural and functional plaque properties.

Chapter 1 is the general introduction to the studies discussed in this thesis. In the first part of this thesis, I focused on subclinical structural and functional changes of the CCA wall and of atherosclerosis at the level of the CCA. Compared to the carotid bifurcation, the CCA has the simple configuration of a straight vessel, running parallel to the skin 
surface and being located rather superficially, thus facilitating US investigation of the artery. Commercially available US systems use B-mode images to measure structural properties such as intima-media thickness (IMT). B-mode however is subjected to various processing steps to reduce noise and improve image quality. As an alternative, unprocessed radio frequency (RF) data can also be used. In Chapter 2, I compared these US techniques to measure CCA-IMT in 136 patients. Although the methods correlated well and had similar reproducibility, B-mode CCA-IMT was significantly larger than the RF-based measurements (mean difference of $45 \mu \mathrm{m}$ ). When analyzing what factors produced this discrepancy, we noticed that vessel wall irregularity (investigated with the RF-based technique using $12 \mathrm{M}$-lines lines covering $13.9 \mathrm{~mm}$ of vessel wall) was one of three contributors. I hypothesized that such spatial inhomogeneity of CCA-IMT could possibly reflect more advanced atherosclerosis, which I further explored in Chapter 3 . In 147 patients, I used the same RF-based measurements and confirmed that spatial IMT-inhomogeneity as well as variation of IMT between measurements were associated with the extent of atherosclerotic disease at the carotid bifurcation. The latter was more strongly associated with severity of atherosclerosis. These results encouraged us to next investigate the spatial variation of functional vessel wall properties. Distension is the change in vessel diameter over the cardiac cycle as function of the change in pulse pressure. Chapter 4 describes the study in which I investigated the spatial distension variation along the CCA in in 154 patients. Plaque at the carotid bifurcation was weakly associated with reduced local absolute and relative distension. However, large inter-measurement variations in distension were strongly associated with distally located stenosis, independent of age, gender and pulse pressure. These results imply that distension inhomogeneity is a marker of atherosclerotic changes within the vessel wall. In Chapter $\mathbf{5}$ we developed a novel method to simultaneously study structural and functional vessel wall properties, as well as functional plaque behavior. From the Plaque At RISK (PARISK) baseline cohort, 23 patients with plaque at the CCA were selected. In routine B-mode US loops, distension at the luminal and adventitial transitions was studied along the local vessel axis instead of a plane perpendicular to the transducer surface. This approach had the advantage to overcome a loss in depth resolution when plaques are not aligned parallel to the transducer surface. At the level of the maximal plaque thickness, the distension curve was used to estimate plaque compression. Though vessel diameters could be measured with good precision, distension and plaque compression were not accurately detected. One observation however was that structural plaque composition was significantly associated with lumen distension: plaques which were echogenic were stiffer compared to plaques which were echolucent.

In the second part of the thesis, the focus is shifted from the CCA to atherosclerotic plaques at the carotid artery bifurcation. Chapter 6 investigated the effect of ageing on the accuracy of duplex US, which is the most commonly used imaging modality to depict 
the carotid bifurcation in patients with recent ischemic stroke. Duplex US uses stringent absolute cut-off values for the peak-systolic blood flow velocity at the level of stenosis, whereas blood flow velocities are known to be inversely associated with increasing age. In 465 arteries free of atherosclerosis, I first confirmed that peak-systolic and enddiastolic velocities in the CCA and internal carotid artery (ICA) linearly decrease with age. Next, I analyzed whether strict cut-off values result in different degrees of stensosis when compared to age-dependent cut-off criteria in 774 arteries with atherosclerotic plaques. Handling strict cut-off values resulted in a difference in the degree of stenosis in both the young (an overestimation of stenosis severity) and the old (an underestimation of stenosis severity). The total number of misclassifications was however small (14 of 774 arteries) and these results need verification in a prospective cohort with CTA or MRA as a reference.

In Chapter 7, I propose novel approach to simultaneously assess structural and functional plaque properties. I manually drew ROIs enclosing all plaque segments, and studied changes of the dimensions of these ROls between the peak-systolic and enddiastolic B-mode frames. In 25 patients from the baseline PARISK cohorts, reproducibility for structural plaque parameters was good to excellent, whereas diastolic-to-systolic change in plaque thickness was moderate reproducible and precise. Other functional parameters (i.e. change in plaque length and movement of plaque center of gravity) could not be measured reproducibly or precisely. In another group of 18 patients, I performed histological validation using plaques that were excised during carotid endarterectomy. On histology, the percentage of lipid core/intraplaque haemorrhage was associated with change in plaque thickness and inversely associated with echogenicity GSM. Therefore, I conclude that simultaneous assessment of structural and functional plaque parameters using routine B-mode US is feasible. Using baseline data from all participants that underwent both US and carotid MRI within the PARISK project, in Chapter 8 I developed a diagnostic prediction rule combining clinical variables and routine B-mode US variables to estimate the probability of finding intraplaque hemorrhage on carotid MRI. This MRI marker is considered to be a strong predictor of future stroke in both symptomatic and asymptomatic subjects. Independent predictors were age, sex, hypertension, echogenicity, stenosis degree and diastolic-to-systolic change in plaque thickness. Combining these predictors showed good discrimination and calibration, but these results need to be replicated and externally validated before the novel PARISK-US score can be implemented in clinical practice to select patients eligible for MRI.

Chapter 9 contains the general discussion of this thesis, in which the results are put in perspective, clinical implications are discussed and I reflect on possible directions for future research. 


\section{Samenvatting}

Een ischemische beroerte, ook wel aangeduid met de term herseninfarct, ontstaat wanneer de aanvoerende slagader afgesloten raakt, wat leidt tot beschadiging van het achterliggende hersenweefsel en verlies van neurologische functie(s) tot gevolg kan hebben. Wereldwijd zijn ischemische beroertes een belangrijke aanslag op de gezondheidszorg. In 2016 werden naar schatting 13,7 miljoen mensen getroffen door een herseninfarct en overleden er 5,5 miljoen mensen aan een herseninfarct. In Nederland zijn in 2017 ruim 29 duizend patiënten opgenomen vanwege een herseninfarct.

Aderverkalking ter hoogte van de splitsing van de halsslagader (arteria carotis) is een belangrijke oorzaak van herseninfarcten. Door het scheuren van het kapsel van bindweefsel dat over een dergelijke atherosclerotische plaque ligt, komt de trombogene inhoud van de plaque in contact met het circulerende bloed. Dit veroorzaakt trombose in het lumen, van waaruit embolieën naar de cerebrale circulatie kunnen migreren. Zodoende ondergaan alle patiënten met een herseninfarct beeldvorming van de carotiden om de aanwezigheid van atherosclerose van de carotiden vast te stellen. Om het risico van nieuwe herseninfarcten in de toekomst in te schatten en daarmee beslissingen omtrent de behandeling van patiënten met herseninfarcten te sturen, wordt in de huidige richtlijnen geadviseerd om de ernst van de vernauwing als gevolg van de atherosclerose te gebruiken als de belangrijkste maat om patiënten te selecteren voor revascularisatie van de arteria carotis. Deze risicostratificatie slaagt er echter niet in om patiënten te identificeren die het meeste zullen profiteren van dergelijke ingrepen. De voorspellende waarde van de mate van stenose voor het bepalen van het risico op een recidief herseninfarct is beperkt. Door vergroten van ons begrip van de processen die uiteindelijk resulteren in ruptuur van de plaque, kan de selectie van patiënten in de toekomst worden verbeterd. Belangrijke factoren waarvan wordt verondersteld dat ze bijdragen aan ruptuur van de plaque zijn de samenstelling van de plaque, de mate van inflammatie van de plaque, en de biomechanische krachten die op de plaque inwerken.

Echografie van de arteria carotis is een niet-invasieve techniek die gelijktijdig plaque samenstelling (structurele eigenschappen) en biomechanische krachten op de plaque (functionele eigenschappen) kan onderzoeken in patiënten die recent een herseninfarct doormaakten. Dit proefschrift heeft tot doel bij te dragen aan het ontwikkelen en valideren van echotechnieken die in vivo bestudering van structurele en functionele eigenschappen van de vaatwand en van de plaque mogelijk maken, om zodoende meer inzicht te genereren in de processen die leiden tot plaque ruptuur. Hiertoe heb ik de volgende doelstellingen geformuleerd:

- Het evalueren van verschillende echotechnieken die de structurele en functionele eigenschappen van de vaatwand in de gemeenschappelijke halsslagader (arteria carotis communis, ACC) en van de atherosclerotische plaque ter hoogte van de interne halsslagader (arteria carotis interna, $\mathrm{ACl}$ ) onderzoeken; 
- Inzicht verwerven in het samenspel tussen structurele en functionele eigenschappen van de CCA-vaatwand en meer distaal gelegen plaque ter hoogte van de carotisbifurcatie en $\mathrm{ACl}$;

- De ontwikkelde echotechnieken gebruiken om gelijktijdig de structurele en functionele plaque eigenschappen te bestuderen in de anatomisch meer complexe carotisbifurcatie.

Hoofdstuk 1 omvat de algemene inleiding op de studies die ik in dit proefschrift bespreek. In het eerste deel van het proefschrift heb ik mij gericht op subklinische structurele en functionele veranderingen van de vaatwand van de ACC en van atherosclerose in de ACC. Vergeleken met de carotisbifurcatie heeft de ACC een eenvoudige configuratie: het is een recht vat dat parallel loopt aan het huidoppervlak en die oppervlakkig gelegen is. Deze kenmerken vergemakkelijken echo-onderzoek van deze slagader.

Commercieel beschikbare echoapparatuur gebruikt de zogenaamde B-mode om structurele vaatwand eigenschappen zoals de intima-media dikte (IMD) te meten. Het B-mode beeld wordt echter aan een aantal bewerkingsstappen onderworpen ten einde ruis te verminderen en de beeldkwaliteit te verbeteren. In plaats van B-mode kan men ook onbewerkte radiofrequentie data gebruiken. In Hoofdstuk 2 heb ik deze twee echotechnieken vergeleken om de IMD van de ACC te meten bij 136 patiënten. Hoewel de twee methoden goed overeenkwamen en vergelijkbare reproduceerbaarheid hadden, was B-mode ACC-IMD significant groter dan de op de RF-methode gebaseerde metingen (gemiddeld verschil van $45 \mu \mathrm{m}$ ). Bij nadere analyse naar factoren die deze discrepantie veroorzaakten, merkten wij op dat een onregelmatige vaatwand één van de drie factoren was die aan het verschil tussen de B-mode en RF-gebaseerde metingen bijdroeg. Deze ruimtelijke inhomogeniteit onderzochten we met de RF-gebaseerde techniek door middel van $12 \mathrm{M}$-lijnen over een afstand van 13,9 mm van de vaatwand. We veronderstelden dat ruimtelijke inhomogeniteit van de ACC-IMD mogelijk meer gevorderde atherosclerose zou kunnen weerspiegelen, wat ik verder heb onderzocht in Hoofdstuk 3. Bij 147 patiënten gebruikten wij dezelfde RF-methode en bevestigden dat zowel ruimtelijke IMT-inhomogeniteit als variatie in IMT tussen twee opeenvolgende metingen geassocieerd waren met de mate van atherosclerose ter hoogte van de carotisbifurcatie. Dit laatste was het sterkst geassocieerd met de ernst van atherosclerose. Deze resultaten moedigden ons aan om vervolgens de ruimtelijke variatie van functionele vaatwandeigenschappen te onderzoeken. Distensie is de verandering in diameter van een bloedvat over de hartcyclus als functie van de verandering in polsdruk. Hoofdstuk 4 beschrijft het onderzoek waarin ik de ruimtelijke inhomogeniteit van distensie in de ACC onderzocht bij 154 patiënten. De mate van atherosclerose ter hoogte van de carotisbifurcatie was slechts zwak geassocieerd met een afname van absolute en relatieve distensie. Verschillen tussen de twee achtereenvolgende metingen waren echter sterk geassocieerd met de ernst van meer distaal gelegen atherosclerose, onafhankelijk 
van leeftijd, geslacht en polsdruk. Deze resultaten impliceren dat inhomogeniteit van distensie een marker kunnen zijn van atherosclerotische veranderingen van de vaatwand. In Hoofdstuk $\mathbf{5}$ ontwikkelden we een nieuwe methode om gelijktijdig structurele en functionele eigenschappen van de vaatwand en van de plaque te bestuderen. Uit het baseline-cohort van de Plaque At RISK (PARISK) studie selecteerden wij 23 patiënten met een plaque in de ACC. In B-mode namen wij korte opnames van de beweging van de vaatwand. Deze opnames gebruikten we om de distensie te bepalen ter hoogte van de luminale en de adventitia overgangen langs de lengteas van het bloedvat, in plaats van loodrecht op het oppervlak van de echotransducer. Deze nieuwe benadering heeft als voordeel dat het verlies aan diepteresolutie wordt beperkt indien plaques niet parallel lopen aan het transducer oppervlak. Ter hoogte van de maximale plaquedikte gebruikten wij de distensiecurve om plaquecompressie te bepalen. Hoewel wij de vaatdiameters met een grote precisie konden meten, waren distensie en plaquecompressie niet nauwkeurig genoeg te detecteren. Wij observeerden echter dat de samenstelling van de structurele plaque significant geassocieerd was met de luminale distensie: echorijke plaques waren stijver in vergelijking met echoarme plaques.

In het tweede deel van dit proefschrift verschuift de aandacht van de AC naar atherosclerotische plaques ter hoogte van de carotisbifurcatie. In Hoofdstuk $\mathbf{6}$ onderzocht ik het effect van veroudering op de accuratesse van duplex van de halsslagaders, wat in de praktijk de meest gebruikte techniek is om atherosclerose ter hoogte van de carotisbifurcatie op te sporen bij patiënten met een recent herseninfarct. De duplex techniek hanteert strikte absolute grenswaarden voor de systolische bloedstroomsnelheden ter plaatste van de stenose. Deze bloedstroomsnelheden zijn echter negatief geassocieerd met een stijgende leeftijd. Als eerste bevestigde ik in 465 arteriën zonder atherosclerose dat de piek-systolische en eind-diastolische bloedstroomsnelheden in de $\mathrm{ACC}$ en $\mathrm{ACl}$ in een lineaire relatie dalen met een stijgende leeftijd. Daarna heb ik geanalyseerd of er een andere stenosegraad zou worden vastgesteld wanneer er gebruik werd gemaakt van leeftijdsafhankelijke afkapwaarden in plaats van de strikte criteria, in 774 slagaders met atherosclerotische plaques. Het hanteren van strikte afkapwaarden resulteerde in een verschil in de mate van stenose bij zowel jongeren (een overschatting van de ernst van de stenose) als de ouderen (een onderschatting van de ernst van de stenose). Het totale aantal misclassificaties was echter klein (bij 14 van de 774 slagaders). Deze resultaten dienen te worden geverifieerd in een prospectief cohort waarin de leeftijdsafhankelijke criteria worden vergeleken met CTA of MRA als referentiestandaard.

In Hoofdstuk 7 stel ik een nieuwe benadering voor om tegelijkertijd structurele en functionele plaque-eigenschappen te beoordelen. Door middel van handmatig intekenen van alle plaquesegmenten op het echobeeld tijdens pieksystole en einddiastole, konden wij de verandering van de afmetingen van deze segmenten bestuderen. Bij 25 deelnemers uit de PARISK studie was de reproduceerbaarheid voor structurele plaque parameters 
goed tot uitstekend, terwijl de verandering van plaquedikte over de hartcyclus matig reproduceerbaar en nauwkeurig was. Andere functionele plaquekenmerken (verandering van plaquelengte en beweging van het zwaartepunt) konden niet reproduceerbaar of nauwkeurig gemeten worden. In een tweede groep van 18 PARISK deelnemers kon ik de verschillende plaquekenmerken histologisch valideren door deze te vergelijken met de pathologische specimen die werden verkregen na carotis endarteriëctomie. Hieruit bleek dat het percentage van de plaque dat bestond uit lipidekern al dan niet met intraplaque bloeding was geassocieerd met verandering van plaquedikte en dat dit negatief was geassocieerd met echogeniciteit. Hieruit concludeerde ik dat gelijktijdige beoordeling van structurele en functionele plaquekenmerken met behulp van commercieel beschikbare echoapparaten mogelijk is. Gebruikmakend van de baseline gegevens van alle deelnemers aan de PARISK studies die zowel een echo als MRI van de halsvaten hadden ondergaan, ontwikkelde ik in Hoofdstuk 8 een diagnostische predictiescore. Deze PARISK-US score combineert klinische variabelen en plaque kenmerken gemeten met routine B-mode echografie om de kans op aanwezigheid van een intraplaque bloeding op MRI te voorspellen. Intraplaque bloeding gevonden bij MRI van de carotiden is een sterke voorspeller voor toekomstige herseninfarcten bij zowel asymptomatische als symptomatische personen. De volgende kenmerken bleken onafhankelijke voorspellers voor de aanwezigheid van intraplaque bloeding: leeftijd, geslacht, hypertensie, echogeniciteit, ernst van de stenose en verandering in plaquedikte over de hartcyclus. Combinatie van deze voorspellers in de PARISK-US score had een goede discriminatie en kalibratie. Deze resultaten moeten echter gerepliceerd en extern gevalideerd worden, voordat de PARISK-US score in de klinische praktijk kan worden geïmplementeerd om patiënten te selecteren die in aanmerking komen voor MRI van de halsvaten.

Hoofdstuk 9 bevat de algemene discussie van dit proefschrift, waarin de resultaten van de verschillende hoofdstukken in perspectief worden geplaatst. Verder bespreek ik klinische implicaties en mogelijke richtingen voor toekomstig onderzoek. 


\section{Valorization}

\section{Relevance}

Ischemic stroke is a major burden of morbidity and mortality. In 2018, a total of 29,419 patients were admitted to the hospital for ischemic stroke in the Netherlands, of which 5,365 patients died.' Besides the devastating effect of ischemic stroke on a patient's personal life, stroke also has a significant impact on health care expenses. Direct health care costs associated with (all-cause) stroke in the Netherlands in 2017 were 1.48 billion euro, being $1.8 \%$ of the total Dutch health care budget, which is in line with estimates from other Western countries. ${ }^{2} 3$ Total costs are even higher because indirect societal costs are not accounted for, such as loss of productivity of patients and caregivers. Thus, reducing the impact that stroke has on society is of great importance.

Carotid atherosclerosis is an importance cause for ischemic stroke. Selecting patients for carotid revascularization treatment needs improvement. Increasing our understanding in the process of plaque destabilization resulting in plaque rupture will help to improve treatment stratification. This thesis aims to contribute by using carotid ultrasonography as a non-invasive imaging technique to gain more insight in the pathophysiology of plaque development and plaque rupture. We developed and validated techniques for assessment of structural and functional vessel wall and plaque properties.

\section{Target groups}

The results of this thesis may be of interest for several target groups. First, clinicians involved in the care for patients with recent ischemic stroke are an important target group. This includes neurologists, vascular surgeons and radiologists, amongst others. Currently, patients with mild to moderate degree of luminal stenosis will not undergo carotid surgery, while some patients may have a stroke in the (near) future. Moreover, asymptomatic subjects will rarely undergo surgery in the Netherlands, while in some of these subjects disabling strokes may be prevented. Improving risk stratification means that patients who will benefit most from revascularization are more likely to be selected for such interventions, whereas subjects who will not benefit or who may even be harmed from surgery will not be referred. This will involve both patients with recent ischemic stroke as well as subjects with asymptomatic carotid artery disease. Second, an important target group will be manufacturers of ultrasonography devices. If simultaneous assessment of structural and functional plaque properties would result in improved risk stratification, this will lead to an increased interest of ultrasonography investigations for patients with carotid artery disease.

\section{Innovation, products and implementation}

The main result of this thesis are that simultaneously investigating structural and functional vessel wall and plaque properties in patients with recent ischemic stroke is 
feasible and reproducible. Moreover, assessment of plaque properties can be used to reliably identify patients with carotid intraplaque hemorrhage. The developed algorithm used to analyze carotid ultrasonography is currently not protected by a patent. The algorithm may however be further improved, e.g. automatic region-of-interest selection instead of manual drawing, before such a patent may be of value.

To result in clinical implementation, several important steps need to be taken. First, results of the PARISK-US score will need to be externally validated in another population of patients with recent stroke, such as the CAPIAS study. Second, the results from the PARISK longitudinal study will need to demonstrate that non-invasive imaging can help to predict future stroke recurrence. More importantly, these results will clarify whether ultrasonography can directly contribute to improved risk stratification, or just through improved selection of patients eligible for carotid MRI. Third, manufacturers of ultrasonography devices may be approached to incorporate data analysis during data acquisition as to facilitate interpretation. Last, to include such approaches in clinical guidelines, trial data need to underline the value of image-based patient selection for carotid revascularization. This will ultimately result in accurate, individualized risk assessment and improved use of carotid revascularization compared to current clinical practice.

\section{References}

1. de Boer AR, Van Dis I, Visseren FL, Vaartjes I, Bots ML. Hart- en vaatziekten in nederland 2019, cijfers over prevalentie, ziekte en sterfte. Den Haag: Hartstichting; 2019.

2. Rijksinstituut voor Volksgezondheid en Milieu. Kosten van ziekten 2017. https://statline.rivm.nl/\#/ RIVM/nl/dataset/50050NED/table?ts=1598696326692. Accessed on August 29, 2020.

3. Evers SM, Struijs JN, Ament AJ, van Genugten ML, Jager JH, van den Bos GA. International comparison of stroke cost studies. Stroke. 2004;35:1209-1215 


\section{Dankwoord}

Aan het tot stand komen van dit proefschrift hebben velen een grote of kleinere bijdrage geleverd. Graag wil ik iedereen bedanken die hier in enige mate aan heeft bijgedragen. De volgende personen wil ik in het bijzonder bedanken.

Allereerst bedank ik alle patiënten die hebben deelgenomen aan één van de hoofdstukken beschreven in dit proefschrift. Het is meer dan bijzonder dat elk van jullie bereid was om extra onderzoeken te ondergaan, en voor PARISK zelfs een hele dag extra naar het ziekenhuis te komen. Velen hadden nog maar kort tevoren een herseninfarct doorgemaakt. Zonder jullie bereidwilligheid was dit proefschrift er niet geweest.

Mijn promotoren en copromotor wil ik bedanken voor hun vertrouwen, steun maar bovenal het geduld dat nodig was om dit proefschrift in de huidige vorm te kunnen aanschouwen.

Prof. dr. W.H. Mess, beste Werner, jij bent betrokken geweest vanaf het prille begin van mijn wetenschappelijke carrière: vanaf de eerste keer dat wij op jouw kamer spraken over intima-media dikte. Ik heb veel van je geleerd over ultrageluid, over research, maar vooral ook veel over het belang van het leven buiten het ziekenhuis. Op mysterieuze wijze vliegt de tijd voorbij als ik bij je langskom!

Prof. dr. M.E. Kooi, beste Eline, onder jouw vleugels heb ik bij de radiologie mogen werken. Ik heb onvoorstelbaar veel van je geleerd over MRI. Jouw gedrevenheid en oog voor detail hebben ertoe geleid dat ik trots kan zijn op het proefschrift dat hier nu ligt, maar jij was ook degene die regelmatig informeerde naar hoe het met me ging. Bedankt voor alles.

Prof. dr. R.J. van Oostenbrugge, beste Robert, vele oud-promovendi hebben jou al geroemd om jouw scherpe wetenschappelijke blik, efficiëntie en kennis. Naast promotor was je ook opleider gedurende het grootste deel van mijn opleiding tot neuroloog en in dat kader maakte je ooit de vergelijking met een herberg. Ik ben dankbaar dat ik zo lang in die herberg heb mogen verblijven: een aangenamer waard tref je zelden.

De leden van de beoordelingscommissie, prof. dr. W.H. Backes, prof. dr. H. ten Cate, prof. dr. C.L. de Korte, prof. dr. C.J.M. Klijn, en prof. dr. G.W.H. Schurink bedank ik graag voor het beoordelen van mijn proefschrift.

Jarenlang ben ik te gast geweest op de afdeling Klinische Neurofysiologie van het MUMC+. De uitjes en het sinterklaasfeest waren legendarisch. Maar bovenal vonden daar vrijwel alle metingen plaats die in dit proefschrift worden beschreven. Daarom wil ik Chantal, Gertie, José, Linda, Mariëlle, Nathal, Odette, Paul en Saskia bijzonder bedanken voor de honderden duplexen waarbij ik jullie tot wanhoop dreef om die plaque nog net wat beter in beeld te brengen. 
Onderzoek doe je nooit alleen, en ik heb het geluk gehad om al die jaren telkens in heel fijne teams te mogen werken. In chronologische volgorde:

Evelien, Julia en Koen: dank voor de (ultragelul) basis! Arnold: als ik ook maar de helft snap van wat jij (in onverkapt Brabants) vertelt, dan mag ik me gelukkig prijzen.

Het Maastrichtse PARISK team. Martine: van buurvrouw, anios, promovendus, neuroloog in opleiding tot paranimf: we hebben veel samen gedeeld. Ik prijs mezelf gelukkig dat jij de basis voor PARISK hebt gelegd: het was een gespreid bedje. Raf en Jeire: ontelbaar veel uren brachten we samen op de MRI- c.q. echokamer door, waaruit een mooie vriendschap is voortgekomen. Raad de MRI sequentie is nu fietsen en samen (sushi) eten geworden. Robert: dank voor je pre-PARISK basis. Stefan: jouw humor miste ik al snel na je vertrek naar Londen. Jolene ("Choline") van Dolly Parton zal nooit meer hetzelfde klinken. Géneviève: nogmaals mijn excuses dat het zorgvuldig opgemaakte bed iets rommeliger was bij jouw start, maar desondanks heb jij PARISK in Maastricht tot een goed einde weten te brengen: dank voor je geduld! Joachim en Paul: bedankt voor het urenlange speuren naar nevenbevindingen. Verder gaat mijn dank ook uit naar de neurologen die patiënten verwezen voor deelname aan PARISK: in het bijzonder Fergus, Narender en Tobien ("senior"). Tiny, bedankt voor je bijdrage aan PARISK maar ook aan alle andere trials.

Multicenter onderzoek kan een uitdaging zijn, maar binnen PARISK bleek niets minder waar. PARISK (oud-)promovendi: Alexandra, Anja, Anouk en Madieke. We komen elkaar op de gekste plaatsen weer tegen en altijd is het als vanouds gezellig. Dank dat ik mocht aansluiten bij dit al hechte team. Ook de vele senior onderzoekers van PARISK, en dan in het bijzonder Aad, Aart, Jaap, Jeroen, Judith, Mat, Paul, Peter en Sylvia: bedankt dat ik een onderdeel van PARISK mocht uitmaken.

Maastricht ICH team: een uit de hand gelopen klinische vraag leidde tot heuse onderzoeksgroep. Julie: bedankt dat je ondanks je drukke schema tijd voor mijn pril ontwaakte interesse wist vrij te maken. Zonder jouw steun werkte ik nu niet in Nijmegen. Linda: bij elke MRI die ik bekijk hoor ik jouw stem in mijn hoofd (erwtensoep of kippensoep?): dank voor je excellente onderwijs in de radiologie! Rik en Kim: elk gingen we onze eigen weg, maar nog altijd waardeer ik ons werk samen! Angel, Bianca, Kirsten, Lisa en Danny: ik voelde me vereerd dat ik jullie mocht begeleiden bij jullie eerste stappen in de wetenschap.

Radboud ICH team: na 15 jaar Maastricht maakte ik de overstap naar Nijmegen. Het is een stap waar ik nog geen spijt van heb gehad. Beste Karin, ik kan je niet genoeg bedanken voor het vertrouwen dat je in me stelt, en het geduld dat je voor me hebt. Jouw tomeloze energie en leiderschap zijn zo inspirerend! Ik hoop dat we nog lang samen kunnen werken aan alle aspecten die intracerebrale bloedingen zo interessant maken! En ik hoop dat je het me vergeeft dat ik je niet bij elk van de volgende teams ga noemen... Janneke, ik heb heel veel van je geleerd over het doen van multicenter onderzoek, en onze trips voor TRIDENT waren altijd gezellig. Ik spijt me zeer dat je niet langer deel kan 
zijn van ons team. Rinske, je bent in korte tijd uitgegroeid tot betrokken, hardwerkende en betrouwbare spil in ons bloedingenteam, en ik kijk uit om met je samen te werken in de lopende en toekomstige studies!

DIST team: Ruben, Jeroen en Peter. Het is heel bijzonder om deel uit te mogen maken van deze samenwerking tussen neurologen en neurochirurgen. We zijn echt anders, maar vullen elkaar goed aan. Ik heb veel respect voor jullie harde werk. Beste Lotte, Jij bent de eerste promovendus die ik mag begeleiden. Vreemd genoeg heb ik soms de indruk dat jij mij coacht. Dank je voor jouw doortastendheid, werkplezier en dat je altijd voor me klaar staat (zowel in werk als privé).

Inflammatie team: Beste Maaike, ook al is jouw promotie pas net gestart, jouw enthousiasme werkt zo aanstekelijk. Zelfs een gebroken arm houdt je niet tegen. Ik ben ervan overtuigd dat we samen CHIPS en ACTION tot een succes gaan maken!

CAA onderzoeksteam: Marcel, Bea, Marieke, Lieke en Anna. Op naar betere diagnostiek voor sporadische CAA! En dat we nog maar vaak samen naar het CAA congres mogen reizen. Anna: dank voor je harde werk aan het BIONIC project. Aan jouw zorg durf ik mijn patiënten echt toe te vertrouwen. Marcel, ik zal die keer dat je voor het eerst bij een LP was in 25 jaar biomarker onderzoek nooit vergeten!

APACHE-AF team: Bart, Berber en Koen. Na jarenlange inspanningen nadert de APACHE-AF zijn afronding. Ik wil jullie bedanken voor de effectieve, maar vooral ook prettige samenwerking die nodig is om een trial in 16 Nederlandse ziekenhuizen te kunnen verrichten. Alle lokale hoofdonderzoekers en onderzoeksverpleegkundigen uit de deelnemende centra: bedankt voor jullie jarenlange inzet om deze trial tot een succes te maken!

FETCH team: Wilmar, Merel, Marieke. Dank jullie dat ik deel mocht nemen aan dit belangrijke onderzoek naar de oorzaken voor hersenbloedingen. Ik hoop dat we veel kunnen leren van de verzamelde gegevens.

HTA team: Janneke, Maroeska, Kas, Mirre en Marike. Dankzij jullie enthousiasme raakte ik snel een (klein) beetje thuis in de theorie van kosteneffectiviteitsonderzoek. Dat jullie de DIST kozen als case voor SURGE voelt als een grote eer.

Run-DMC/inTENse team: Kim, Annemieke, Esther, Mina, Anil en Frank-Erik. Ik heb veel respect voor de wijze waarop jullie je inzetten voor deze uitzonderlijke studie. Kim, samen microbloedingen scoren was altijd gezellig, ook al kregen we er soms vierkante ogen van.

Cerebellair ICH team: Bart en Sanjula. Mooi om samen de diagnostiek, prognose en behandeling van dit type bloedingen onder de loep te nemen. Bedankt dat ik hier aan mag bijdragen.

Lastly, I would like to thank the international collaborators and colleagues, in particular Adrian, Caoimhe, Charlotte, Craig, Grace, Jamie, Nesh, Rustam and Ruth. 
Graag wil ik de stafleden en (oud)aios neurologie van het MUMC+ bedanken voor de jarenlange fijne omgeving waarin ik het vak van neuroloog mocht leren. In het bijzonder Caroline, Ellen, Ellen, Hanneke, Janneke, Jessie, Kim, Laura, Martijn, Mayienne, Pim, Rik en Tom: of we elkaar nu spreken op de Biemond of belanden in een intercollegiaal overleg, het voelt altijd alsof we de draad gewoon weer oppakken! Daarnaast wil ik ook Peter van Domburg bedanken voor zijn inspirerende inwijding in de neurologie.

Zoals gezegd was de overstap van Maastricht naar Nijmegen een grote sprong voor een geboren Limburger, maar dankzij de stafleden en (oud)aios neurologie van het Radboudumc voelde ik mij direct thuis. Ik heb respect voor al jullie harde werk en ben er trots op dat we ondanks onze verschillende interesses toch zo'n hecht team vormen. Extra trots ben ik natuurlijk op ons vasculaire speerpunt: Anil, Edo, Ewoud, Frank-Erik, en Karin (alweer!). Elk van ons heeft zijn eigen kwaliteiten en we komen uit bijna alle windstreken, maar samen zijn we een ijzersterk team. Dank jullie dat ik als laatste hierbij mocht aansluiten! Ook Karin, Saskia en Sharon wil ik graag bedanken voor het plezier waarmee jullie je inzetten: ik loop altijd graag even bij jullie binnen. Anton en Sjoert, Jeroen, Joost en Saman: zonder goede neuroradiologen en neurochirurgen zijn wij neurologen reddeloos verloren; bedankt dat jullie zo goed zijn in jullie vak maar bovenal dat het zo prettig met jullie samenwerken is!

Met de steun van vrienden en familie ben ik zover gekomen. In de eerste plaats dank ik mijn adoptie-jaarclub Chapeau. Erik en Nicole, Erwin en Zoë, Roel en Ayaka, Sander en Mareille, Sonny en Ingeborg, en Thijs en Jade. Ik waardeer onze vriendschap enorm. En wie had ruim 18 jaar geleden ooit durven denken dat we zeven zulke mooie dames aan onze club zouden mogen toevoegen. Ik hoop jullie na covid-19 allemaal bij ons thuis te kunnen uitnodigen om het afronden van mijn proefschrift te vieren (jullie hebben zo vaak mijn gezeur moeten aanhoren). Fijn om te weten dat het zwaartepunt van onze wijdverspreide woonplaatsen in Weert ligt!

ledereen die deel uit maakt van de skiclub (onmogelijk om iedereen te noemen vanwege de sterk wisselende samenstelling): bedankt voor dit jaarlijkse hoogtepunt! Van Tignes tot Westendorf, van team pussy tot team pain, jägerbomben en steak haché: elk van de trips waar we mee mochten waren legendarisch.

Een speciaal woord voor Yvette. Jij bent sinds onze verhuizing naar Weert lid geworden van ons gezin, want zo voelt het voor Nienke en mij. De kinderen zijn gek op je! En dankzij jou had ik de rust om die laatste "punten en komma's" te zetten: mijn dank is groot!

Lieve Klaas en Widelo: bedankt dat jullie de liefste schoonouders zijn die ik me kan wensen. Sinds onze eerste kennismaking voel ik me thuis bij jullie gezin. Ook jullie hebben me regelmatig geholpen om te kunnen schrijven, door jullie studeerkamer in Empe voor 
me beschikbaar te stellen waar ik geholpen door het schitterende uitzicht kon werken aan dit proefschrift. Mijn schoonzussen en -broers, Joppe en Mels, Rixt en Nico: ook jullie wil ik graag bedanken voor de gezelligheid en warmte van jullie familie waarvan ik al vele jaren onderdeel mag zijn. Een speciaal woord van dank gaat uit naar Rixt. Toen een collega opmerkte dat het schilderij dat jij voor ons huis in Maastricht had gemaakt wel erg leek op bloedvaten, had ik niet kunnen bedenken dat ik de kaft van mijn proefschrift al aan de muur had hangen. Dank je voor de vele uren die je hebt gestoken in de vormgeving van mijn proefschrift. Zonder jou was het lang niet zo mooi geworden!

Lieve pappa en mamma. Nu ik zelf ouder van twee zonen ben, realiseer ik me pas hoe belangrijk een goede basis is. Van jongs af aan hebben jullie me op de juiste momenten gestimuleerd, altijd achter me gestaan en me mijn eigen keuzes laten maken. Jullie hebben me geleerd dat er hard gewerkt moet worden om ergens te komen, maar ook dat genieten en ontspannen belangrijk is. Al blijf ik dat laatste moeilijk vinden. Dank jullie voor alles! Jan en Tobias: mijn twee "brothers in crime”. We hebben zo ontzettend veel samen meegemaakt, en ook al zijn we niet van het dagelijks bellen, ik denk wel elke dag aan jullie. Jullie steun is heel belangrijk voor me. En Tobias: dat ik het je de eerste jaren niet zo makkelijk heb gemaakt, betaalt zich nu terug: ik ben heel blij dat jij op deze dag naast me wilt staan. Cathelijne en Saar: ik ben zo blij dat mijn broers een partner hebben gevonden, waarmee het zo goed klikt binnen ons gezin. Het maakt niet uit waar of in welke samenstelling we elkaar treffen, maar het is altijd zo gezellig. Nu we elk ons eigen gezin hebben, merk ik dat deze band een nieuw tijdperk ingaat. Ik hoop dat we nog vele jaren samen op Schier van ons kroost kunnen genieten!

Lieve Berend, bedankt dat je zo'n ongelooflijk lieve knuffelkont bent, en ik ben blij dat ik je kan zeggen: pappa's boek is (eindelijk) klaar! Kleine Pepijn, jij hebt gelukkig niet veel meegekregen van al dit werk, maar bedankt dat je altijd zo vrolijk bent!

Allerliefste Nienke, vanaf het allereerste begin dat we samen zijn, ben ik met dit onderzoek bezig geweest. Jouw liefde, steun en praktische hulp heeft me door alle ups en downs getrokken die onderzoek doen met zich meebrengt. Dat je dat ruim veertien jaar hebt volgehouden, is een bewijs voor jouw geduld (dus toch!) en liefde. Gekscherend heb ik het soms over ons derde kind, die onderhand al een puber zou zijn. Zonder jou was dit proefschrift er zeker niet geweest. Nu kan ik echt zeggen: het is af. Bedankt dat je er altijd voor me bent! Ik hou zielsveel van jou. 


\section{List of publications}

This thesis

1. Schreuder FHBM, Mess WH, Hoeks APG. Ageing affects the accuracy of duplex ultrasonography in grading carotid artery stenosis. Cerebrovascular Diseases. 2009;27:75-83

2. Graf IM, Schreuder FHBM, Hameleers JM, Mess WH, Reneman RS, Hoeks APG. Wall irregularity rather than intima-media thickness is associated with nearby atherosclerosis. Ultrasound in Medicine \& Bbiology. 2009;35:955-961

3. Schreuder FHBM, Graf M, Hameleers JM, Mess WH, Hoeks APG. Measurement of common carotid artery intima-media thickness in clinical practice: Comparison of B-mode and RF-based technique. Ultraschall in der Medizin/European Journal of Ultrasound. 2009;30:459-465

4. Graf IM, Schreuder FHBM, Mess WH, Reneman RS, Hoeks APG. Spatial distension variations are associated with focal atherosclerotic plaques. Cerebrovascular Diseases. 2010;29:199-205

5. Steinbuch J, Schreuder FHBM, Reesink KD, Hoeks APG, Mess WH. Orthogonal B-mode evaluation of common carotid artery plaques reveals the absence of outward remodeling. Ultrasound in Medicine \& Biology. 2018;44:986-994

\section{Other publications}

1. Schreuder FHBM, Hoeks APG, Mess WH. Inadequate acoustical temporal bone window in patients with transient ischemic attack or minor stroke: Role of skull thickness and bone density. Ultrasound in Medicine \& Biology. 2009;35:1229; author reply 1230

2. Kwee RM, van Engelshoven JM, Mess WH, ter Berg JW, Schreuder FHBM, Franke CL, Korten AG, Meems BJ, van Oostenbrugge RJ, Wildberger JE, Kooi ME. Reproducibility of fibrous cap status assessment of carotid artery plaques by contrast-enhanced MRI. Stroke. 2009;40:3017-3021

3. Kwee RM, Schreuder FHBM, Mess WH, van Oostenbrugge RJ, Triebels VH, van den Akker LH, Heeneman S, Hofman PA, van Engelshoven JM, Wildberger JE, Kooi ME. Images in cardiovascular medicine: Development of carotid intraplaque hemorrhage demonstrated by serial magnetic resonance imaging. Circulation. 2009;120:1637-1639

4. Schreuder FHBM, Henskens LH. [Diagnostic image. A man with tongue deviation following a motorcycle accident]. Nederlands Tijdschrift voor Geneeskunde. 2010;154:A175

5. Schreuder FHBM, Hamers RM, van Domburg PH. Teaching neuroimages: Holmes tremor after midbrain stroke. Neurology. 2010;75:e10

6. Truijman MTB, Kooi ME, van Dijk AC, de Rotte AA, van der Kolk AG, Liem MI, Schreuder FHBM, Boersma E, Mess WH, van Oostenbrugge RJ, Koudstaal PJ, Kappelle LJ, Nederkoorn PJ, Nederveen AJ, Hendrikse J, van der Steen AFW, Daemen MJAP, van der Lugt A. Plaque At Risk (PARISK): Prospective multicenter study to improve diagnosis of high-risk carotid plaques. International Journal of Stroke. 2014;9:747-754

7. Schols AM, Schreuder FHBM, van Raak EP, Schreuder TH, Rooyer FA, van Oostenbrugge RJ, Staals J. Incidence of oral anticoagulant-associated intracerebral hemorrhage in the Netherlands. Stroke. 2014;45:268-270

8. van 't Klooster R, Truijman MTB, van Dijk AC, Schreuder FHBM, Kooi ME, van der Lugt A, van der Geest RJ. Visualization of local changes in vessel wall morphology and plaque progression in serial 
carotid artery magnetic resonance imaging. Stroke. 2014;45:e160-163

9. Truijman MTB, de Rotte AA, Aaslid R, van Dijk AC, Steinbuch J, Liem MI, Schreuder FHBM, van der Steen AFW, Daemen MJAP, van Oostenbrugge RJ, Wildberger JE, Nederkoorn PJ, Hendrikse J, van der Lugt A, Kooi ME, Mess WH. Intraplaque hemorrhage, fibrous cap status, and microembolic signals in symptomatic patients with mild to moderate carotid artery stenosis: The Plaque At Risk study. Stroke. 2014;45:3423-3426

10. de Greef BT, Schreuder FHBM, Vlooswijk MC, Schreuder AH, Rooyer FA, van Oostenbrugge RJ, Rouhl RP. Early seizures after intracerebral hemorrhage predict drug-resistant epilepsy. Journal of Neurology. 2015;262:541-546

11. de Rotte AA, Truijman MTB, van Dijk AC, Liem MI, Schreuder FHBM, van der Kolk AG, et al. Plaque components in symptomatic moderately stenosed carotid arteries related to cerebral infarcts: The Plaque At Risk study. Stroke. 2015;46:568-571

12. Parry-Jones AR, Di Napoli M, Goldstein JN, Schreuder FHBM, Tetri S, Tatlisumak T, Yan B, van Nieuwenhuizen KM, Dequatre-Ponchelle N, Lee-Archer M, Horstmann S, Wilson D, Pomero F, Masotti L, Lerpiniere C, Godoy DA, Cohen AS, Houben R, Al-Shahi Salman R, Pennati P, Fenoglio L, Werring D, Veltkamp R, Wood E, Dewey HM, Cordonnier C, Klijn CJM, Meligeni F, Davis SM, Huhtakangas J, Staals J, Rosand J, Meretoja A. Reversal strategies for vitamin K antagonists in acute intracerebral hemorrhage. Annals of Neurology. 2015;78:54-62

13. Liem MI, Schreuder FHBM, van Dijk AC, de Rotte AA, Truijman MTB, Daemen MJAP, van der Steen AFW, Hendrikse J, Nederveen AJ, van der Lugt A, Kooi ME, Nederkoorn PJ. Use of antiplatelet agents is associated with intraplaque hemorrhage on carotid magnetic resonance imaging: The Plaque At Risk study. Stroke. 2015;46:3411-3415

14. Behrouz R, Azarpazhooh MR, Godoy DA, Hoffmann MW, Masotti L, Parry-Jones AR, Popa-Wagner A, Schreuder FHBM, Slevin MA, Smith CJ, Di Napoli M. The multi-national survey on epidemiology, morbidity, and outcomes in intracerebral haemorrhage (MNEMONICH). International Journal of Stroke. 2015;10:E86

15. Di Napoli M, Zha AM, Godoy DA, Masotti L, Schreuder FHBM, Popa-Wagner A, Behrouz R. Prior cannabis use is associated with outcome after intracerebral hemorrhage. Cerebrovascular Diseases. 2016;41:248-255

16. Steinbuch J, Hoeks APG, Hermeling E, Truijman MTB, Schreuder FHBM, Mess WH. Standard B-mode ultrasound measures local carotid artery characteristics as reliably as radiofrequency phase tracking in symptomatic carotid artery patients. Ultrasound in Medicine \& Biology. 2016;42:586-595

17. Frusch KJ, Houben R, Schreuder FHBM, Postma AA, Staals J. Association between eye position on brain scan and hospital mortality in acute intracerebral hemorrhage. European Journal of Neurology. 2016;23:831-835

18. Vöö S, Kwee RM, Sluimer JC, Schreuder FHBM, Wierts R, Bauwens M, Heeneman S, Cleutjens JP, van Oostenbrugge RJ, Daemen JW, Daemen MJAP, Mottaghy FM, Kooi ME. Imaging intraplaque inflammation in carotid atherosclerosis with 18F-fluorocholine positron emission tomographycomputed tomography: Prospective study on vulnerable atheroma with immunohistochemical validation. Circulation. Cardiovascular imaging. 2016;9:e004467

19. Steinbuch J, van Dijk AC, Schreuder FHBM, Truijman MTB, de Rotte AAJ, Nederkoorn PJ, van der Lugt A, Hermeling E, Hoeks APG, Mess WH. High spatial inhomogeneity in the intima-media thickness of the common carotid artery is associated with a larger degree of stenosis in the internal carotid artery: 
The PARISK study. Ultraschall in der Medizin/European Journal of Ultrasound. 2017;38:523-529

20. Schreuder FHBM, Sato S, Klijn CJM, Anderson CS. Medical management of intracerebral haemorrhage. Journal of Neurology, Neurosurgery and Psychiatry. 2017;88:76-84

21. van Hoof RHM, Schreuder FHBM, Nelemans PJ, Truijman MTB, van Orshoven NP, Schreuder TH, Mess WH, Heeneman S, van Oostenbrugge RJ, Wildberger JE, Kooi ME. Ischemic stroke patients demonstrate increased carotid plaque microvasculature compared to (ocular) transient ischemic attack patients. Cerebrovascular Diseases. 2017;44:297-303

22. van Hoof RHM, Vöö SA, Sluimer JC, Wijnen NJA, Hermeling E, Schreuder FHBM, Truijman MTB, Cleutjens JPM, Daemen MJAP, Daemen JH, van Oostenbrugge RJ, Mess WH, Wildberger JE, Heeneman S, Kooi ME. Vessel wall and adventitial DCE-MRI parameters demonstrate similar correlations with carotid plaque microvasculature on histology. Journal of Magnetic Resonance Imaging. 2017;46:1053-1059

23. Steinbuch J, van Dijk AC, Schreuder FHBM, Truijman M, Hendrikse J, Nederkoorn PJ, van der Lugt A, Hermeling E, Hoeks APG, Mess WH. Definition of common carotid wall thickness affects risk classification in relation to degree of internal carotid artery stenosis: The Plaque At Risk (PARISK) study. Cardiovascular Ultrasound. 2017;15:9

24. Wilson D, Seiffge DJ, Traenka C, Basir G, Purrucker JC, Rizos T, Sobowale OA, Sallinen H, Yeh SJ, Wu TY, Ferrigno M, Houben R, Schreuder FHBM, Perry LA, Tanaka J, Boulanger M, Al-Shahi Salman R, Jäger HR, Ambler G, Shakeshaft C, Yakushiji Y, Choi PMC, Staals J, Cordonnier C, Jeng JS, Veltkamp R, Dowlatshahi D, Engelter ST, Parry-Jones AR, Meretoja A, Werring DJ. Outcome of intracerebral hemorrhage associated with different oral anticoagulants. Neurology. 2017;88:1693-1700

25. Klijn CJM, Schreuder FHBM. In patients with intracerebral haemorrhage and concomitant atrial fibrillation, optimal timing of reinitiating anticoagulants may be 7-8 weeks after ICH. Evidence Based Medicine. 2017;22:108-109

26. Di Napoli M, Behrouz R, Topel CH, Misra V, Pomero F, Giraudo A, Pennati P, Masotti L, Schreuder FHBM, Staals J, Klijn CJM, Smith CJ, Parry-Jones AR, Slevin MA, Silver B, Willey JZ, Azarpazhooh MR, Vallejo JM, Nzwalo H, Popa-Wagner A, Godoy DA. Hypoalbuminemia, systemic inflammatory response syndrome, and functional outcome in intracerebral hemorrhage. Journal of Critical Care. 2017;41:247-253

27. Claessens D, Bekelaar K, Schreuder FHBM, de Greef BTA, Vlooswijk MCG, Staals J, van Oostenbrugge RJ, Rouhl RPW. Mortality after primary intracerebral hemorrhage in relation to post-stroke seizures. Journal of Neurology. 2017;264:1885-1891

28. Houben R, Schreuder FHBM, Bekelaar KJ, Claessens D, van Oostenbrugge RJ, Staals J. Predicting prognosis of intracerebral hemorrhage: Performance of ICH score is not improved by adding oral anticoagulant use. Frontiers in Neurology. 2018;9:100

29. van Middelaar T, Argillander TE, Schreuder FHBM, Deinum J, Richard E, Klijn CJM. Effect of antihypertensive medication on cerebral small vessel disease: A systematic review and metaanalysis. Stroke. 2018;49:1531-1533

30. Crombag GAJC, Spronk HM, Nelemans PJ, Schreuder FHBM, Truijman MTB, van Dijk AC, de Rotte AAJ, Liem MI, Daemen MJAP, van der Steen AFW, Mess WH, Nederkoorn PJ, Hendrikse J, van der Lugt A, Wildberger JE, Ten Cate H, van Oostenbrugge RJ, Kooi ME. No association between thrombin generation and intra-plaque haemorrhage in symptomatic carotid atherosclerotic plaques: The Plaque At Risk (PARISK) study. Thrombosis and haemostasis. 2018;118:1461-1469 
31. Tsivgoulis G, Wilson D, Katsanos AH, Sargento-Freitas J, Marques-Matos C, Azevedo E, Adachi T, von der Brelie C, Aizawa Y, Abe H, Tomita H, Okumura K, Hagii J, Seiffge DJ, Lioutas VA, Traenka C, Varelas P, Basir G, Krogias C, Purrucker JC, Sharma VK, Rizos T, Mikulik R, Sobowale OA, Barlinn K, Sallinen H, Goyal N, Yeh SJ, Karapanayiotides T, Wu TY, Vadikolias K, Ferrigno M, Hadjigeorgiou G, Houben R, Giannopoulos S, Schreuder FHBM, Chang JJ, Perry LA, Mehdorn M, Marto JP, Pinho J, Tanaka J, Boulanger M, Al-Shahi Salman R, Jäger HR, Shakeshaft C, Yakushiji Y, Choi PMC, Staals J, Cordonnier C, Jeng JS, Veltkamp R, Dowlatshahi D, Engelter ST, Parry-Jones AR, Meretoja A, Mitsias PD, Alexandrov AV, Ambler G, Werring DJ. Neuroimaging and clinical outcomes of oral anticoagulant-associated intracerebral hemorrhage. Annals of Neurology. 2018;84:694-704

32. Freeze WM, Jacobs HIL, Schreuder FHBM, van Oostenbrugge RJ, Backes WH, Verhey FR, Klijn CJM. Blood-brain barrier dysfunction in small vessel disease related intracerebral hemorrhage. Frontiers in Neurology. 2018;9:926

33. van Dijk AC, Donkel SJ, Zadi T, Sonneveld MAH, Schreuder FHBM, Chohan MF, Koudstaal PJ, Leebeek FWG, Saxena R, Hendrikse J, Kooi ME, van der Lugt A, de Maat MPM. Association between fibrinogen and fibrinogen gamma' and atherosclerotic plaque morphology and composition in symptomatic carotid artery stenosis: Plaque At Risk study. Thrombosis Research. 2019;177:130-135

34. Crombag GAJC, Schreuder FHBM, van Hoof RHM, Truijman MTB, Wijnen NJA, Vöö SA, Nelemans PJ, Heeneman S, Nederkoorn PJ, Daemen JH, Daemen MJAP, Mess WH, Wildberger JE, van Oostenbrugge RJ, Kooi ME. Microvasculature and intraplaque hemorrhage in atherosclerotic carotid lesions: A cardiovascular magnetic resonance imaging study. Journal of Cardiovascular Magnetic Resonance. 2019;21:15

35. Crombag GAJC, van Hoof RHM, Holtackers RJ, Schreuder FHBM, Truijman MTB, Schreuder AHCML, van Orshoven NP, Mess WH, Hofman PAM, van Oostenbrugge RJ, Wildberger JE, Kooi ME. Symptomatic carotid plaques demonstrate less leaky plaque microvasculature compared with the contralateral side: A dynamic contrast-enhanced magnetic resonance imaging study. Journal of the American Heart Association. 2019;8:e011832

36. Ter Telgte A, Wiegertjes K, Gesierich B, Marques JP, Huebner M, de Klerk JJ, Schreuder FHBM, Araque Caballero MA, Kuijf HJ, Norris DG, Klijn CJM, Dichgans M, Tuladhar AM, Duering M, de Leeuw FE. Contribution of acute infarcts to cerebral small vessel disease progression. Annals of Neurology. 2019;84:582-592

37. van der Burg S, Schreuder FHBM, Klijn CJMM, Verbeek MM. Valuing biomarker diagnostics for dementia care: Enhancing the reflection of patients, their care-givers and members of the wider public. Medicine, Health Care and Philosophy. 2019;22:439-451

38. Ekker MS, Jacob MA, van Dongen MM, Aarnio K, Annamalai AK, Arauz A, Arnold M, Barboza MA, Bolognese M, Brouns R, Chuluun B, Chuluunbaatar E, Dagvajantsan B, Debette S, Don A, Enzinger C, Ekizoglu E, Fandler-Höfler S, Fazekas F, Fromm A, Gattringer T, Gulli G, Hoffmann M, Hora TF, Jern C, Jood K, Kamouchi M, Kim YS, Kitazono T, Kittner SJ, Kleinig TJ, Klijn CJM, Korv J, Lee TH, Leys D, Maaijwee NA, Martinez-Majander N, Marto JP, Mehndiratta MM, Mifsud V, Montanaro VV, Owolabi MO, Patel VB, Phillips MC, Piechowski-lozwiak B, Pikula A, Ruiz-Sandoval JL, Sarnowski B, Schreuder FHBM, Swartz RH, Tan KS, Tanne D, Tatlisumak T, Thijs V, Tuladhar AM, Viana-Baptista M, Vibo R, Wu TY, Yesilot N, Waje-Andreassen U, Pezzini A, Putaala J, de Leeuw FE. Global outcome assessment life-long after stroke in young adults initiative - the GOAL initiative: Study protocol and rationale of a multicentre retrospective individual patient data meta-analysis. BMJ Open. 2019;9:e031144 
39. Patel A, Schreuder FHBM, Klijn CJM, Prokop M, Ginneken BV, Marquering HA, Roos YBWEM, Baharoglu MI, Meijer FJA, Manniesing R. Intracerebral haemorrhage segmentation in non-contrast CT. Scientific Reports. 2019;9:17858

40. Cockx HM, Meijer FJA, Schreuder FHBM. Uw diagnose? Cerebrale amyloïd angiopathie geassocieerde inflammatie. Tijdschr Neurol Neurochir. 2019;5:198-200

41. Jolink WM, Lindenholz A, van Etten ES, van Nieuwenhuizen KM, Schreuder FHBM, Kuijf HJ, van Osch MJ, Hendrikse J, Rinkel GJ, Wermer MJ, Klijn CJ. Contrast leakage distant from the hematoma in patients with spontaneous ICH: A 7 T MRI study. Journal of Cerebral Blood Flow and Metabolism. 2020;40:1002-1011

42. Sondag L, Schreuder FHBM, Boogaarts HD, Rovers MM, Vandertop WP, Dammers R, Klijn CJM. Neurosurgical intervention for supratentorial intracerebral hemorrhage. Annals of Neurology. 2020;88:239-250

43. Kuiperij HB, Hondius DC, Kersten I, Versleijen AAM, Rozemuller AJM, Greenberg SM, Schreuder FHBM, Klijn CJM, Verbeek MM. Apolipoprotein D: A potential biomarker for cerebral amyloid angiopathy. Neuropathology and Applied Neurobiology. 2020;46:431-440

44. Jäkel L, Kuiperij HB, Gerding LP, Custers E, Van den Berg E, Jolink WM, Schreuder FHBM, Küsters B, Klijn CJM, Verbeek MM. Disturbed balance in the expression of MMP9 and TIMP3 in cerebral amyloid angiopathy-related intracerebral haemorrhage. Acta Neuropathologica Communications. 2020 Jul 6;8(1):99. doi: 10.1186/s40478-020-00972-z

45. Van Nieuwenhuizen KM, Vaartjes I, Verhoeven J, Rinkel GJ, Kappelle JL, Schreuder FHBM, Klijn CJM. Long-term prognosis after intracerebral haemorrhage. European Journal of Stroke. Accepted for publication 
Chapter 10 


\section{About the author}

Floris Hendricus Benjamin Michel Schreuder was born on Friday the 29th of July 1983 in the St. Barbara Hospital in Geleen, the Netherlands. He graduated with honors from the Graaf Huyn College in 2001, after which he started his medical studies in Maastricht. In 2006, he attended a two-month internship in Neurology at the Mater Misericordiae University Hospital in Dublin, Ireland (supervision dr. T. Lynch and dr. P.J. Kelly). Here, his interest in clinical neurology was aroused.

During his last year of medical training. Floris worked as a senior intern at the Department of Neurology of the Maastricht University Medical Center (supervised by dr. A. Twijnstra). He also started a research project on atherosclerosis of the carotid artery at the Departments of Clinical Neurophysiology and Biophysics (prof. dr. W.H. Mess and prof. dr. A.P.G. Hoeks). After he obtained his medical degree in 2007, he continued to work on this project for another six months as a researcher at the Cardiovascular Research Institute Maastricht (CARIM) to lay the basis for this thesis.

Floris worked a year as a resident in Neurology at the Orbis Medical Center, Sittard. In May 2009, he began his registrar training in Neurology at the Maastricht University Medical Center (supervision dr. M. de Krom and prof. dr. R.J. van Oostenbrugge). As part of his training, Floris worked in the Atrium Medical Center Heerlen in 2010 (supervision dr. P.J. Koehler). He interrupted his residency for 18 months to join the PARISK team in 2013-2014 (prof. dr. W.H. Mess, prof. dr. M.E. Kooi and prof. dr. R.J. van Oostenbrugge) and continued working on his thesis. He initiated two other research projects which are not part of this thesis, one on intracerebral hemorrhage and one on wake-up stroke. For the latter project, he won a scholarship from the Royal Dutch Academy of Sciences (KNAW van Leersum beurs) and attended the Universitätsklinikum Gießen und Marburg, Germany (supervision prof. dr. M. Kaps).

Floris completed his residency in Neurology in June 2016, and he now works as a neurologist and clinical researcher with specific interest in intracerebral hemorrhage at the Department of Neurology of the Radboud University Medical Center in Nijmegen. In 2020 he was awarded a Senior Clinical Scientist grant by the Dutch Heart Foundation. Floris has (co)authored nearly 50 scientific articles and currently co-supervises three PhD students.

On a rainy Monday morning in December 2006, Floris met Nienke Wieringa in the Neurology conference room. They got married in 2014 and together they have two sons: Berend and Pepijn. 




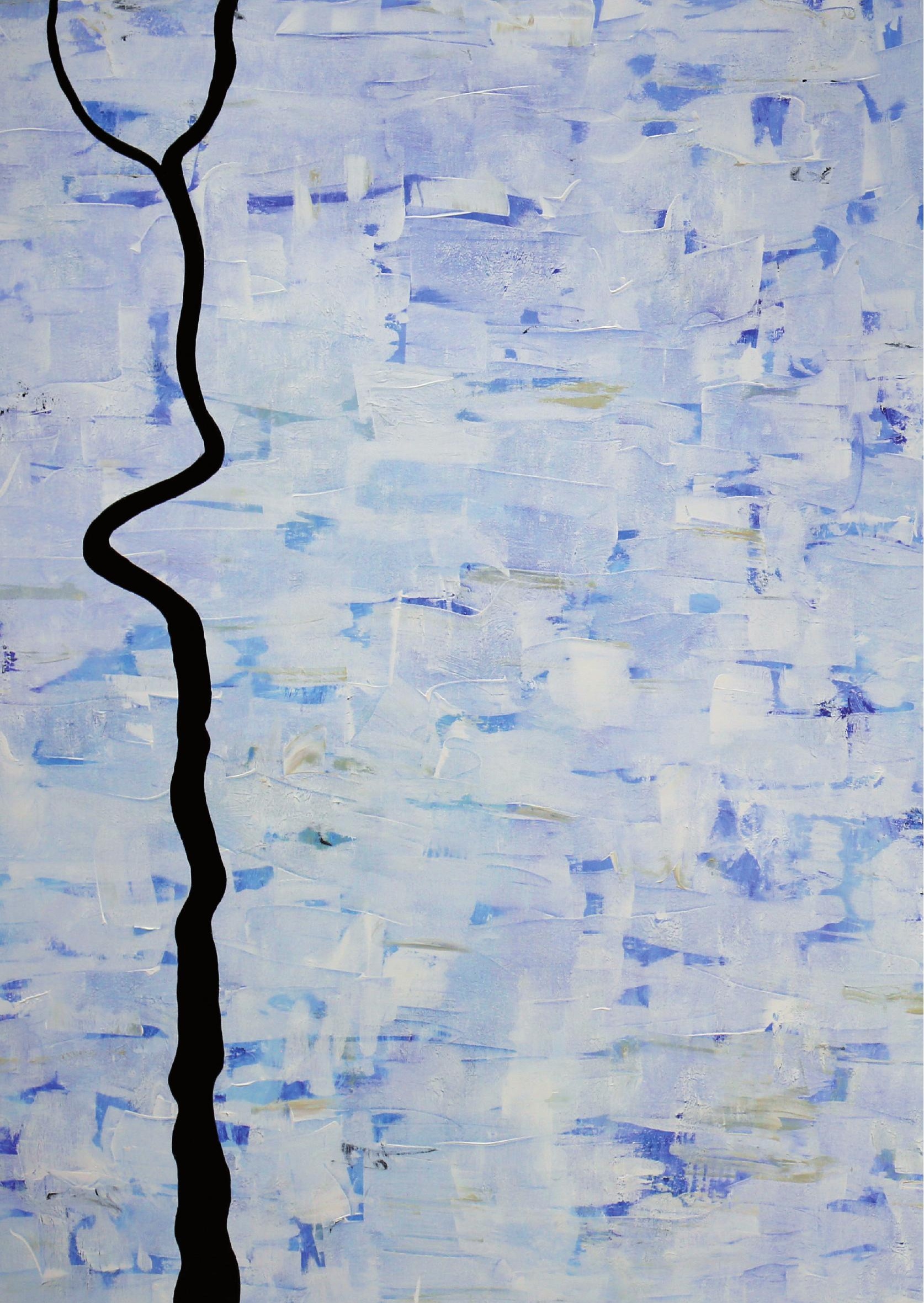

